\title{
Potential lethal and sublethal effects of gypsy moth biological treatments on non-target lepidopterans in two Appalachian forests
}

\author{
Kenneth Edward Rastall \\ West Virginia University
}

Follow this and additional works at: https://researchrepository.wvu.edu/etd

\section{Recommended Citation}

Rastall, Kenneth Edward, "Potential lethal and sublethal effects of gypsy moth biological treatments on non-target lepidopterans in two Appalachian forests" (1999). Graduate Theses, Dissertations, and Problem Reports. 3183.

https://researchrepository.wvu.edu/etd/3183

This Dissertation is protected by copyright and/or related rights. It has been brought to you by the The Research Repository @ WVU with permission from the rights-holder(s). You are free to use this Dissertation in any way that is permitted by the copyright and related rights legislation that applies to your use. For other uses you must obtain permission from the rights-holder(s) directly, unless additional rights are indicated by a Creative Commons license in the record and/ or on the work itself. This Dissertation has been accepted for inclusion in WVU Graduate Theses, Dissertations, and Problem Reports collection by an authorized administrator of The Research Repository @ WVU.

For more information, please contact researchrepository@mail.wvu.edu. 


\title{
Potential Lethal and Sublethal Effects of Gypsy Moth Biological Treatments on Non-target Lepidopterans in Two Appalachian Forests
}

\author{
Kenneth Edward Rastall \\ Dissertation submitted to the College of Agriculture, \\ Forestry and Consumer Sciences, Division of \\ Plant and Soil Sciences at West Virginia University \\ in partial fulfillment of the requirements for the degree of
}

Doctor of Philosophy

in

Agricultural Sciences - Entomology

Linda Butler, Ph.D., Chair

James Amrine, Ph.D.

Henry Hogmire, Ph.D.

Andrew Liebhold, Ph.D.

Thomas Pauley, Ph.D.

Morgantown, West Virginia

1999

Keywords: Non-target, Bacillus thuringiensis, macrolepidoptera, sublethal Copyright 1999 Kenneth Edward Rastall 


\title{
ABSTRACT \\ Potential Lethal and Sublethal Effects of Gypsy Moth Biological Treatments on Non-target Lepidopterans in Two Appalachian Forests
}

\author{
Kenneth E. Rastall
}

Eighteen 200 ha plots were established in the Monongahela National Forest (MON), Pocahontas County, West Virginia, and in the George Washington National Forest (GW), Augusta County, Virginia. From 1995 through 1998 black light traps, foliage pruning, and canvas bands were used to collect macrolepidopteran adults and larvae. During 1997 and 1998, 6 plots each were aerially treated with nucleopolyhedrosis virus (Gypchek $^{\mathrm{TM}}$; GC) and Bacillus thuringiensis var. kurstaki (Btk: Bt). The remaining 6 plots were left untreated (Ref). Leaf samples from $B t$ plots were analyzed for toxin concentrations using enzyme-linked immunosorbant assay techniques. Results indicated concentrations above $20 \mathrm{ng} / \mathrm{cm}^{2}$ for $90.1 \%$ of the samples.

Adults and larvae of 19 species from 5 families were tallied to assess potential treatment effects. Weights of larvae (and their pupae) and wing lengths of adults were measured to assess possible sublethal effects. Analysis of variance indicated a significant interaction $(\mathrm{P}<0.05)$ between pretreatment/treatment years and the 3 treatment groups for 2 species of adults and 3 species of larvae. Fewer individuals were collected from $B t$ plots than from GC and Ref plots during treatment years, but not during pretreatment years. A similar pattern was observed for the 19 species combined and for the combined sum of all early season non-target macrolepidopteran larvae collected from foliage. Significant forest differences were also observed, with adults of 9 species and larvae of 6 species more numerous in MON catches. Adults of 1 species were more numerous in GW catches.

Sublethal treatment effects of Btk were not identified; however, significant differences between forests were noted. Adults of 13 species from GW catches possessed longer wings than those collected from the MON. One MON species possessed longer wings. Adult males were significantly more numerous and typically possessed shorter wings than females. Significant fluctuations in counts were the result of variation in the number of males. GW larvae (and their pupae) were significantly heavier than MON larvae for 1 species. A comparison of temperatures between forests indicated significantly cooler temperatures on the MON during pretreatment and treatment years. 


\section{ACKNOWLEDGEMENTS}

This research was made possible by a USDA Forest Service grant, with personal funding provided by a Mellon/Burroughs Welcome Foundation Fellowship from the Appalachian College Association. I am indebted to them both for this funding. I would like to thank the College of Agriculture, Forestry, and Consumer Sciences of West Virginia University (WVU) for providing me with the remarkable opportunity to further my studies. I am grateful to Wheeling Jesuit University and all my colleagues who have been supportive friends and who have provided much inspiration during my continued education.

I am extremely grateful to Dr. Linda Butler, my major professor/advisor, for her friendship and guidance throughout this endeavor. Without her mentorship, helpful advice, and patience, I would not have been able to conduct this research. I would also like to extend my gratitude to Dr. James Amrine, Dr. Henry Hogmire, Dr. Andrew Liebhold, and Dr. Thomas Pauley, who were supportive and helpful while serving on my graduate committee. I am grateful to Dr. Edwin Townsend, and again to Dr. Andrew Liebhold, for their helpful suggestions regarding the statistical analyses included in my dissertation.

I would personally like to thank Dr. John Stazanac for his constant assistance and suggestions regarding practically every aspect of my research in both the laboratory and in the field. I also appreciate his friendship and willingness to open his home to me and making me feel welcome during the tedious driving inherent in the field work. I owe a special debt of gratitude to Vicki Kondo for her enormous contribution in identifying and sorting the thousands of moths used in my study. Her patience in helping me to identify and sort these individuals was wonderfully appreciated. Likewise, I would like to thank Deb Blue for her contribution in the tedious job of sorting and rearing caterpillars. Again, I would like to thank Dr. Butler for her patience and vast expertise in working with the caterpillars and 
identifying them, while both she and Deb Blue remained patient through the additional work of weighing the larvae.

Of the WVU Entomology Department, during all or portions of the study, I would like to thank Greg Chrislip, Changlu Wang, Mike Whitman, Terry Carrington, Toby Petrice, Karen Grubb, Rachael Braud, and the many student workers who assisted in the laboratory and field work. I would like to thank the ornithology crew, University of Georgia, and the herpetology crew, Marshall University, for their friendship and their contributions to the study. I would also extend my gratitude to the Hamden Connecticut Forest Service laboratory and to Pam Huntley for their assistance in conducting $B t$ toxin analysis. I am indebted to the late Dr. Normand Dubois for opening his home to me and offering any and all forms of assistance during my stay in Connecticut and at the Forest Service laboratory.

I would finally like to thank my wife Pamela for her love, support, and patience throughout this entire process. She and our newborn son have provided me with a renewed and optimistic outlook for which I am extremely grateful. 


\section{Table of Contents}

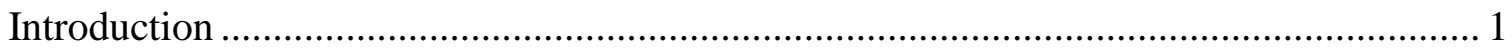

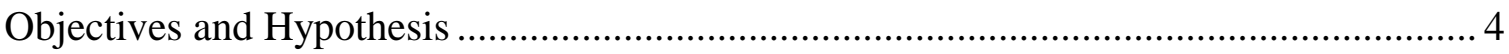

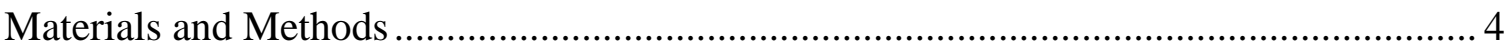

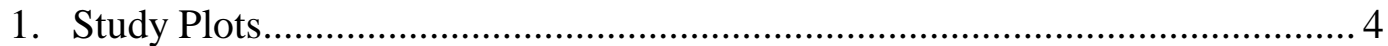

2. Monitoring Physical Parameters ................................................................... 5

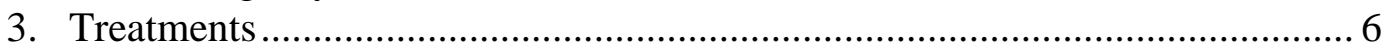

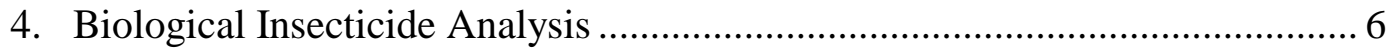

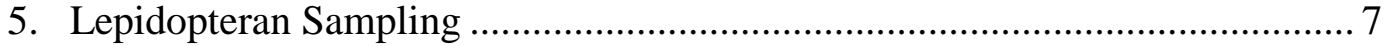

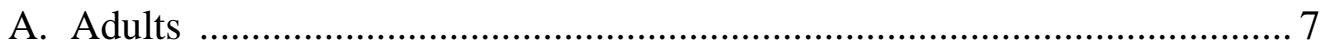

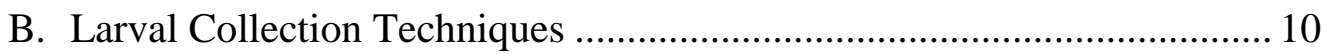

6. Larval Rearing Techniques....................................................................... 11

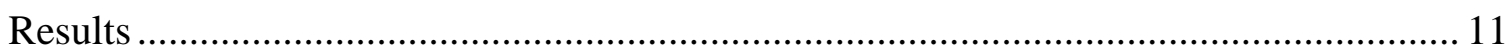

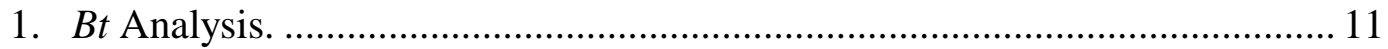

2. Average Weekly Sampling Event Temperatures......................................... 15

3. Statistical Analyses Applied to Selected Lepidopteran Species....................... 17

4. Trends Indicated by Statistical Analyses....................................................... 24

A. Selected Species Combined, geometrids, and noctuids .......................... 25

Larval Counts (1995-1998) ................................................................... 25

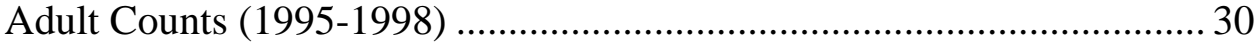

Adult Counts (1995 \& 1998)................................................................ 32

Adult Wing Lengths $(1995$ \& 1998) ..................................................... 35

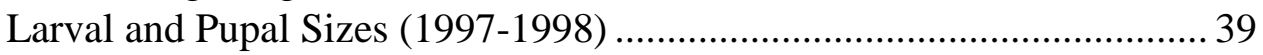

B. Statistical Results for Individual Species ............................................ 42

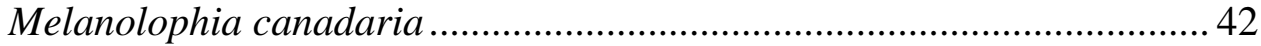

Hypagyrtis unipunctata ............................................................... 47

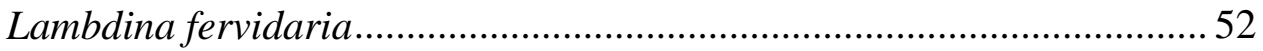

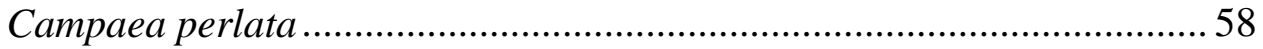

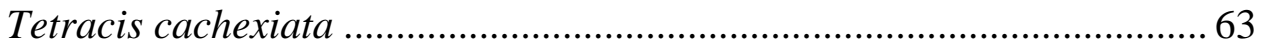

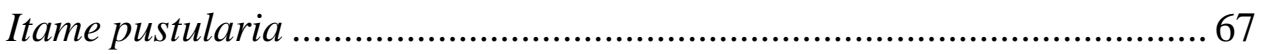

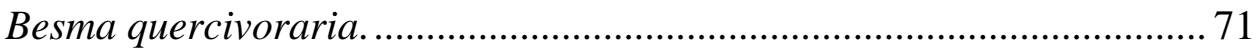

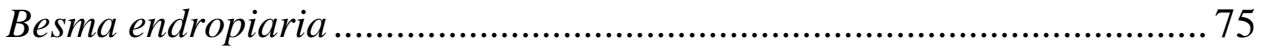

Malacosoma disstria ...................................................................... 81

Heterocampa guttivitta................................................................ 85

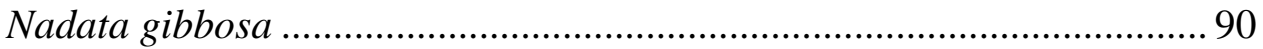

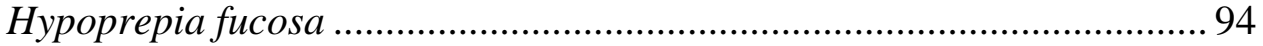

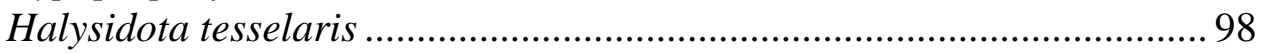

Achatia distincta ............................................................................. 103

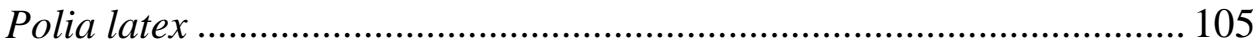




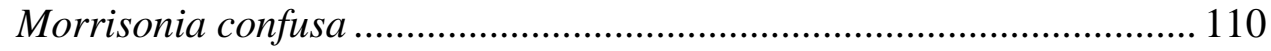

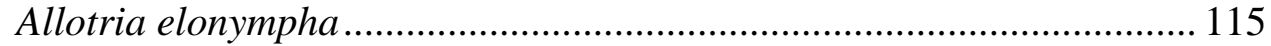

Baileya opthalmica .................................................................... 117

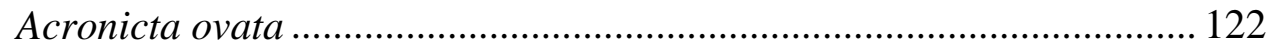

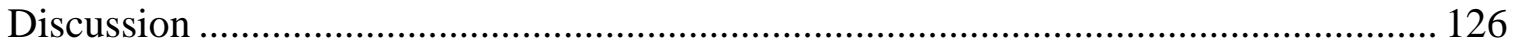

A. Summary of Lepidopteran Statistical Trends ........................................... 126

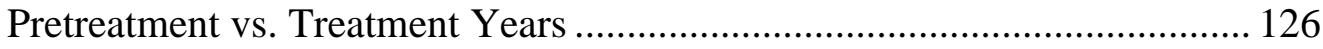

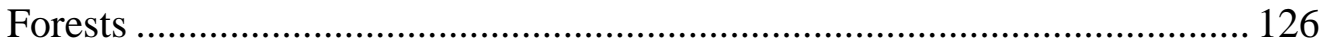

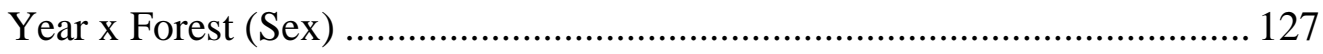

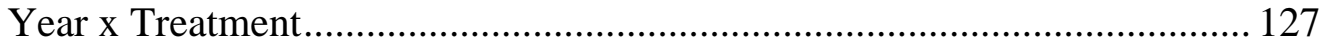

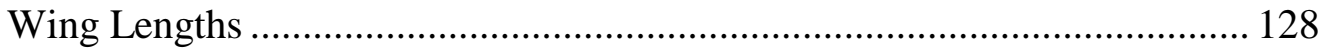

Larval and Pupal Weights ............................................................. 128

B. Implications of Lepidopteran Trends ..................................................... 129

Fluctuations in Population Abundances .................................................. 129

Potential $B t$ Lethal Effects................................................................... 131

Potential Bt Sublethal Effects................................................................. 133

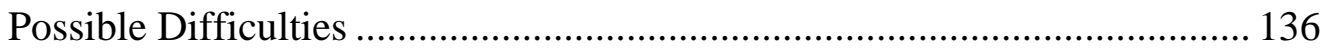

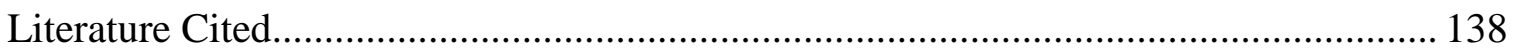

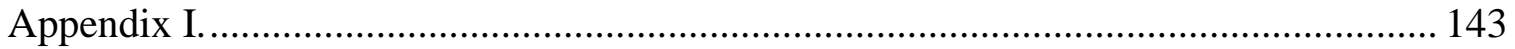

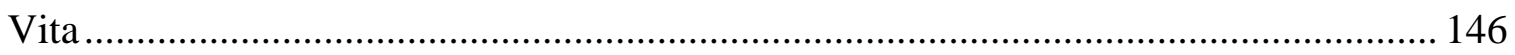




\section{LIST OF TABLES}

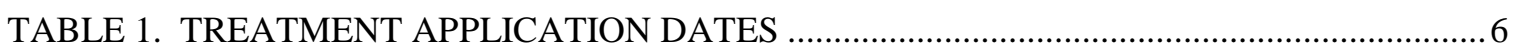

TABLE 2. SELECTED LEPIDOPTERAN FAMILIES AND SPECIES...........................................

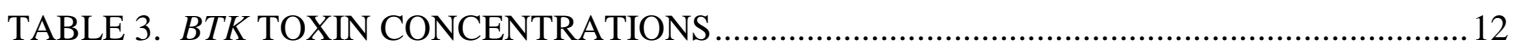

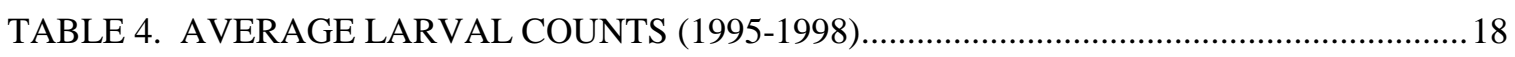

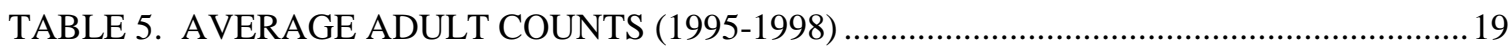

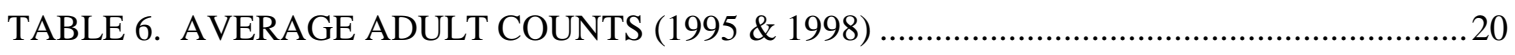

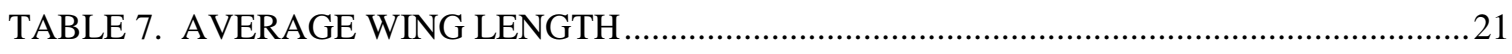

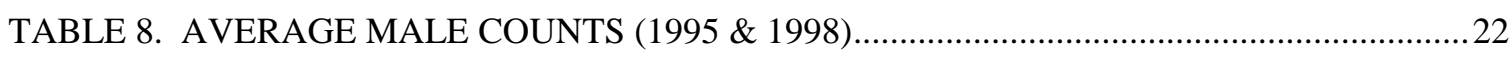

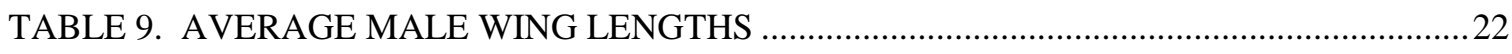

TABLE 10. FEMALE AND MALE ADULT COUNTS AND

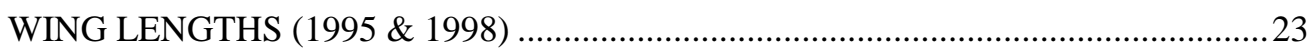

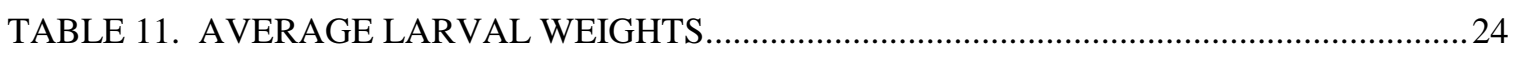

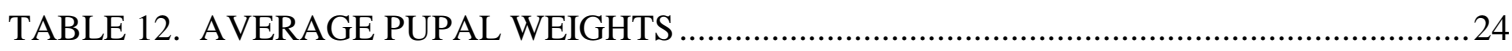




\section{LIST OF FIGURES}

\section{BT CONCENTRATIONS AND TEMPERATURE}

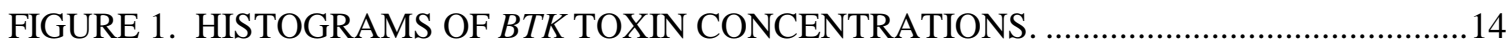

FIGURE 2. AVERAGE TEMPERATURES FOR WEEKLY SAMPLING EVENTS. ....................16

\section{SELECTED SPECIES COMBINED, GEOMETRIDS, AND NOCTUIDS}

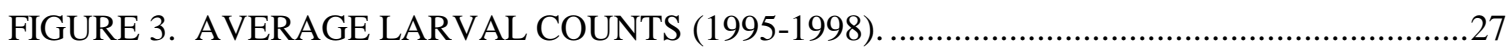

FIGURE 4. AVERAGE LARVAL COUNT INTERATIONS (1995-1998) ....................................28

FIGURE 5. AVERAGE WEEKLY LARVAL COUNTS (1997-1998). .............................................29

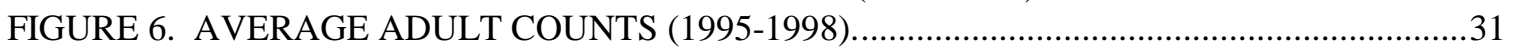

FIGURE 7. AVERAGE FEMALE AND MALE ADULT COUNTS (1995 \& 1998) ..........................33

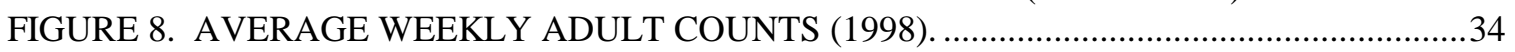

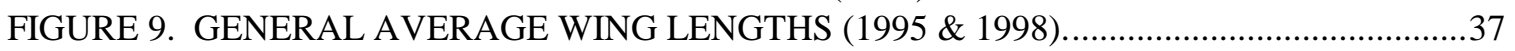

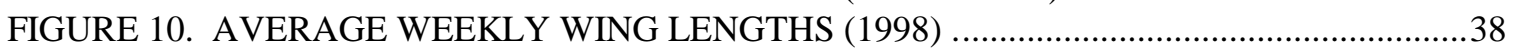

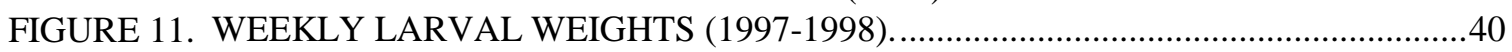

FIGURE 12. AVERAGE LARVAL AND PUPAL WEIGHTS (1997-1998) .....................................41

\section{STATISTICAL RESULTS FOR INDIVIDUAL SPECIES}

\section{Melanolophia canadaria}

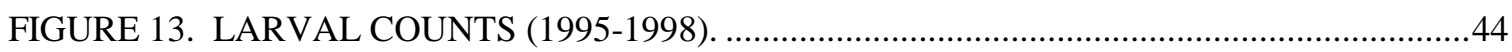

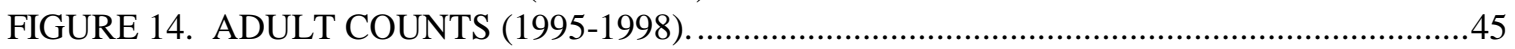

FIGURE 15. FEMALE AND MALE ADULT COUNTS AND WING LENGTHS (1995 \& 1998). 46

\section{Hypagyrtis unipunctata}

FIGURE 16. WEEKLY LARVAL AND ADULT COUNTS (1995-1998). .......................................49

FIGURE 17. LARVAL AND ADULT COUNTS WITH ADULT INTERACTION (1995-1998)...50

FIGURE 18. FEMALE AND MALE ADULT COUNTS AND WING LENGTHS (1995 \& 1998). 51

FIGURE 19. MALE WING LENGTH INTERACTION (1995 \& 1998)............................................52

\section{Lambdina fervidaria}

FIGURE 20. WEEKLY LARVAL AND ADULT COUNTS (1995-1998)......................................55 FIGURE 21. LARVAL AND ADULT COUNTS WITH LARVAL INTERACTION (1995-1998)..56 FIGURE 22. FEMALE AND MALE ADULT COUNTS AND WING LENGTHS (1995 \& 1998). 57 FIGURE 23. WING LENGTH INTERACTION (1995 \& 1998).

\section{Campaea perlata}

FIGURE 24. WEEKLY LARVAL AND ADULT COUNTS (1995-1998)........................................60

FIGURE 25. LARVAL AND ADULT COUNTS (1995-1998) ..................................................61

FIGURE 26. FEMALE AND MALE COUNTS AND WING LENGTHS (1995 \& 1998)................62 


\section{LIST OF FIGURES (CONTINUED)}

\section{Tetracis cachexiata}

FIGURE 27. WEEKLY LARVAL AND ADULT COUNTS (1995-1998), ........................................64

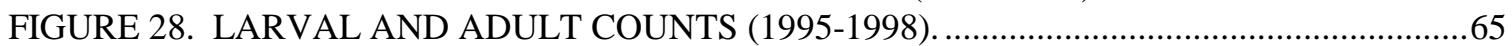

FIGURE 29. FEMALE AND MALE ADULT COUNTS AND WING LENGTHS (1995 \& 1998). 66

\section{Itame pustularia}

FIGURE 30. WEEKLY LARVAL AND ADULT COUNTS (1995-1998), ......................................68

FIGURE 31. LARVAL AND ADULT COUNTS (1995-1998)...................................................69

FIGURE 32. FEMALE AND MALE ADULT COUNTS AND WING LENGTHS (1995 \& 1998). 70

\section{Besma quercivoraria}

FIGURE 33. WEEKLY LARVAL AND ADULT COUNTS (1995-1998)....................................72

FIGURE 34. LARVAL AND ADULT COUNTS (1995-1998)....................................................73

FIGURE 35. FEMALE AND MALE ADULT COUNTS AND WING LENGTHS (1995 \& 1998). 74

\section{Besma endropiaria}

FIGURE 36. WEEKLY LARVAL AND ADULT COUNTS (1995-1998), .....................................77

FIGURE 37. LARVAL AND ADULT COUNTS (1995-1998)....................................................

FIGURE 38. FEMALE AND MALE ADULT COUNTS AND WING LENGTHS (1995 \& 1998). 79

FIGURE 39. AVERAGE LARVAL WEIGHTS.

\section{Malacosoma disstria}

FIGURE 40. WEEKLY LARVAL AND ADULT COUNTS (1995-1998).....................................82

FIGURE 41. ADULT COUNTS AND INTERACTION (1995-1998)............................................83

FIGURE 42. FEMALE AND MALE ADULT COUNTS AND WING LENGTHS (1995 \& 1998). 84

\section{Heterocampa guttivitta}

FIGURE 43. WEEKLY LARVAL AND ADULT COUNTS (1995-1998).....................................87

FIGURE 44. LARVAL AND ADULT COUNTS WITH LARVAL INTERACTION (1995-1998)..88

FIGURE 45. FEMALE AND MALE ADULT COUNTS AND WING LENGTHS (1995 \& 1998)..89

\section{Nadata gibbosa}

FIGURE 46. WEEKLY LARVAL AND ADULT COUNTS (1995-1998)......................................91

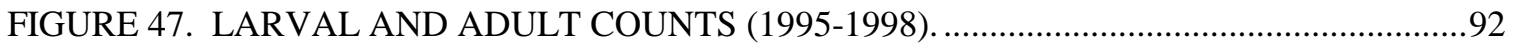

FIGURE 48. FEMALE AND MALE ADULT COUNTS AND WING LENGTHS (1995 \& 1998). 93 


\section{Hypoprepia fucosa}

\section{LIST OF FIGURES (CONTINUED)}

FIGURE 49. WEEKLY LARVAL AND ADULT COUNTS (1995-1998), .....................................95

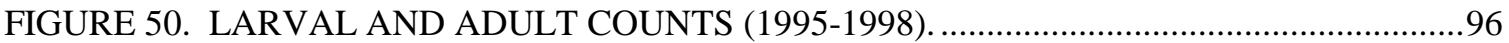

FIGURE 51. FEMALE AND MALE ADULT COUNTS AND WING LENGTHS (1995 \& 1998). 97

\section{Halysidota tesselaris}

FIGURE 52. WEEKLY LARVAL AND ADULT COUNTS (1995-1998).

FIGURE 53. LARVAL AND ADULT COUNTS AND LARVAL INTERACTION (1995-1998). 101

FIGURE 54. FEMALE AND MALE ADULT COUNTS AND

WING LENGTHS (1995 \& 1998)

\section{Achatia distincta}

FIGURE 55. LARVAL INTERACTION AND ADULT COUNTS (1995-1998).

\section{Polia latex}

FIGURE 56. WEEKLY LARVAL AND ADULT COUNTS (1995-1998) .......................................106

FIGURE 57 LARVAL AND ADULT COUNTS (1995-1998)......................................................107

FIGURE 58 FEMALE AND MALE ADULT COUNTS AND WING LENGTHS (1995 \& 1998).108

FIGURE 59 LARVAL AND PUPAL WEIGHTS (1997-1998)...................................................109

\section{Morrisonia confusa}

FIGURE 60 WEEKLY LARVAL AND ADULT COUNTS (1995-1998)

FIGURE 61 LARVAL AND ADULT COUNTS WITH ADULT INTERACTION (1995-1998)...113

FIGURE 62 FEMALE AND MALE ADULT COUNTS AND WING LENGTHS (1995 \& 1998).114

\section{Allotria elonympha}

FIGURE 63 ADULT COUNTS (1995-1998; 1995 \& 1998). 116

\section{Baileya opthalmica}

FIGURE 64 WEEKLY LARVAL AND ADULT COUNTS (1995-1998) ...................................119

FIGURE 65 LARVAL AND ADULT COUNTS (1995-1998)....................................................120

FIGURE 66 FEMALE AND MALE ADULT COUNTS AND WING LENGTHS (1995 \& 1998).121

\section{Acronicta ovata}

FIGURE 67 WEEKLY LARVAL AND ADULT COUNTS (1995-1998).......................................123

FIGURE 68 LARVAL AND ADULT COUNTS (1995-1998).....................................................124

FIGURE 69 FEMALE AND MALE ADULT COUNTS AND WING LENGTHS (1995 \& 1998)......... 125 


\section{Potential lethal and sublethal effects of gypsy moth biological treatments on selected non-target lepidopteran species in two Appalachian forests}

\section{Introduction:}

The relatively recent spread of gypsy moth southward and westward from New England into the Appalachian regions of West Virginia and Virginia has aroused concerns regarding the fate of the hardwood forests present in these areas. Concerns stem primarily from the gypsy moths' reputation for being ravenous defoliators (Andreadis and Weseloh 1990; Roth et al. 1997). Though populations exhibiting outbreak numbers may be subsequently reduced by viruses or, more recently, by fungal epizootics (Hajek et al. 1990b; Hajek et al. 1995), unregulated outbreaks may still potentially last long enough to inflict considerable damage. Unfortunately, attempts to suppress gypsy moth outbreaks with the use of chemical pesticides has had the undesirable effect of impacting non-target organisms. For example, diflubenzuron (Dimilin ${ }^{\mathrm{TM}}$ ), used for a number of years, produces season-long lethal effects to non-target lepidopteran larvae and also affects a variety of other arthropod species (Butler and Kondo 1993; Butler et al. 1997a). A more recently developed chemical, tebufenozide (Mimic $\left.{ }^{\mathrm{TM}}-\mathrm{RH}-5992\right)$, though perhaps more specific, has still been shown to impact non-target macrolepidoptera (Butler et al. 1997b). In an effort to further reduce non-target effects, and still suppress gypsy moth populations, attempts have been made to explore the use of biological sprays containing viruses or bacteria lethal to gypsy moth larvae.

The use of Bacillus thuringiensis var. kurstaki (Btk) and nuclear polyhedrosis virus (NPV; Gypchek $^{\mathrm{TM}}$ )as biological control agents has become a common method for controlling gypsy moths (Podgwaite et al. 1992; Reardon et al. 1994; Liebhold and McManus 1999). However, due to the complex interrelationships among the organisms in an ecosystem, it is important to determine if these biological control agents have unanticipated impacts on non-target organisms. The arthropod communities inhabiting forests and forest canopies are no exception (i.e. Crossley et al. 1976; Overgaard Nielsen \& Ejlersen 1977; Moran and Southwood 1982). Historically, however, studies of the effects of Bacillus thuringiensis (Bt) on non-target insects have been rather limited (Reardon et al. 1994). Studies to date suggest that large scale $B t$ treatments may have effects on some non-target organisms, especially on the Lepidoptera (Miller 1990a,b; Sample et al. 1993; Sample et al. 1996; Wagner et al. 1996). 
Therefore, the primary focus of a long-term, non-target study conducted by West Virginia University (WVU) with cooperation of the USDA Forest Service Forest Health Technology Enterprise Team, Morgantown, USDA Forest Service (Coop No. 42-793), coordinated by Dr. Linda Butler, WVU, has been to determine the potential effects that gypsy moth treatments may have on the large numbers of different nontarget organisms. The study is focused on forest arthropods and some of the vertebrate species that prey upon them (namely salamanders and birds).

The arthropod portion of the field study has included intensive, 15-week sampling periods conducted during the summers of 1995 through 1998. Pretreatment data collected in 1995 and 1996 provided baseline information, while 1997 and 1998 data provided information on two sequential-year applications of biological control agents (Btk and NPV-- Gypchek ${ }^{\mathrm{TM}}$ ). Data collected from subsequent years will ultimately provide additional post treatment information for the long-term study.

In conjunction with the long term study previously mentioned, additional information was collected. Light trap, foliage pruning, and canvas band collection of adult and larval lepidopterans during the 1995, 1996, 1997, and 1998 sampling seasons of the long-term study were used to assess potential lethal and sublethal effects of $B t k(B t)$ and $\operatorname{Gypchek}^{\mathrm{TM}}(\mathrm{GC})$ on 19 of the non-target lepidopteran species which were most commonly obtained. A larger number of species of the families Noctuidae and Geometridae were chosen for analysis, particularly due to their relatively high abundances and that other non-target studies have indicated that these families contain some species which appear to be vulnerable to $B t$ treatments (Butler et al. 1995b).

Potential sublethal effects were assessed by comparing average yearly wing lengths of adults collected from both treatment and non-treatment plots during the 1995 (pre-treatment) and 1998 (treatment) years. Likewise, larval weights were determined for individuals of the same species collected during most of the 1997 sampling year, and all of the 1998 sampling year. Individuals which pupated provided additional information via their weights.

Comparisons of wing lengths and larval/pupal weights of lepidopterans collected from the various plots were based on the premise that a larva ingesting a sublethal dose of these potentially toxic compounds might feed less due to the reduction in a general decline in vigor, or due to paralysis of its digestive tract (Reardon et al. 1994). The toxins from $B t$ typically affect susceptible lepidopteran larvae with a stomach $\mathrm{pH}$ 
above 8.0. Proteolytic enzymes dissolve ingested crystals produced by the $B t$ bacteria. The subsequent release of endotoxins results in ultimate degradation of cells in the midgut. Death may result from gut paralysis. In some lepidopteran larvae, it may not necessarily be the toxin itself which results in larval death but the later infection (septicemia) of opportunistic bacteria following midgut cell lysis and subsequent degradation of the peritrophic membrane (Dubois and Dean 1995). Interestingly, an insect's ability to breakdown the crystals into toxic subunits may dictate which insects are susceptible to $B t$ (Reardon et al. 1994). Gypchek ${ }^{\mathrm{TM}}$ functions as a viral infection, rupturing cells which not only results in death of the target organism, but in further production of viral particles. As a virus, GC appears to be highly specific to gypsy moth larvae as opposed to other lepidopteran larvae tested in the laboratory (Barber et al. 1993).

Certain toxins may cause sudden cessation of feeding behavior of insects post-ingestively, sometimes within a matter of minutes (Bernays and Chapman 1995). Some insects may eventually recover from sublethal doses (Reardon et al. 1994), but ultimately show residual effects. Studies on sublethal effects of $B t$ on spruce budworm showed developmental time significantly increased, ultimately resulting in reduced pupal size and a reduction in the number of eggs laid by the adult females (Pedersen et al. 1997). Males appeared more affected by toxins than females. In other cases, even if toxins are present in lethal quantities, the insect may detect these toxins and avoid them. However, since the $B t$ toxins and GC were sprayed aerially in the described study, it was expected that "clean" foliage might be difficult to find. Some energy expenditure in locating suitable food could thus be expected. Consequently, such individuals might exhibit a reduction in overall size and/or reduced fecundity as well.

Implications of sublethal effects are important not only in potentially reducing fecundity, but also because delayed development might increase susceptibility to predation (Pedersen et al. 1997; Cloutier and Jean 1998). Sublethally debilitated lepidopteran larvae have been known to live as long as a month (Fitzgerald 1995). Delayed development might further cause larvae to miss an opportunity to exploit a particularly important food source (Strong et al. 1984) or increase susceptibility to parasitism (Weseloh 1984). The plant tissue might not only toughen with age, but timing of emergence may be very important to some herbivore specialists that might also be susceptible to certain plant responses to herbivory (Denno et al. 1995). 
To evaluate potential sublethal effects of the biological control agents it was necessary to assess spray coverage homogeneity by determining the concentrations of these agents, especially $B t$ toxins, on the foliage following treatments. Consequently, analysis for these toxins utilizing enzyme-linked immunosorbent assays (ELISA) techniques (Wie et al. 1982) was conducted on preparations obtained from leaves collected on treatment plots immediately following the 1997 and 1998 treatments.

\section{Objectives and Hypotheses:}

1. To determine the potential lethal effects of aerially applied Gypchek ${ }^{\mathrm{TM}}$ (GC) and Bacillus thuringiensis var. kurstaki (Btk) on 19 species of non-target macrolepidoptera.

2. To determine if potential differences in selected species abundances exist within or between two neighboring Appalachian hardwood forests, one mesic and the other xeric.

3. To determine if species-specific size differences exist within or between forests before or after treatment with Btk and GC.

The null hypothesis for the analyses was that no significant differences would exist between forests or among treatments.

\section{Materials and Methods:}

\section{Study Plots}

Each of eighteen 200 ha plots was established in the Monongahela National Forest (MON), Pocahontas County, West Virginia, and in the George Washington National Forest (GW), Augusta County, Virginia. These forests are dominated by tree species typical of mature oak-maple-hickory hardwood forests and exhibit a potential for severe defoliation by gypsy moths. The GW is generally more xeric than the MON.

Of the eighteen plots, 9 (enumerated 1 through 9) were located on the Deerfield Ranger District of the GW along Great North Mountain, Virginia, while the remaining 9 plots (enumerated 10-18) were located on the MON in West Virginia. The West Virginia plots were specifically located in the southern Greenbrier Ranger District on Chestnut Ridge (Paddy Knob North); the Marlington Ranger District near Sugar Camp Run (Paddy Knob South), and in the Marlington Ranger District on Marlin Mountain. The MON plots are located at an average elevation of approximately 950m, while GW plots are located at an average elevation 
of approximately $635 \mathrm{~m}$. The plots provide a completely randomized blocked experimental design based on vegetation type.

\section{Monitoring of Physical Parameters}

Environmental conditions greatly affect insect feeding and host plant selection (as reviewed by Bernays and Chapman 1995). Since insects, as small-bodied poikilotherms are sensitive to these conditions, it is often difficult to separate these factors from potential "treatment" factors. Likewise, physical parameters such as temperature and humidity may affect the efficacy of disease organisms (Hajek et al. 1990a; Hajek and Soper 1992). The two Appalachian forests in this study exhibited potential differences in these parameters. Consequently, in 1995, rain gauges and maximum/minimum mercury thermometers were placed at each plot 6 weeks after the onset of the study. The rain gauges and thermometers were vertically attached to a wooden plaque $1 \mathrm{~m}$ above the ground. The thermometers were placed facing north and a board was attached to the top of the plaque which overlapped slightly for protection and to exclude direct sunlight. 


\section{Treatments}

Within each forest, three plots were randomly designated to be treated with GC, three with $B t$, and three acted as reference (or control) plots and were left untreated (Ref), for 6 of each designation for the 18 plots. Aerial treatment applications were applied to the designated plots in each forest during the Spring of 1997 and 1998 following leaf bud-break, when oak leaves were approximately 1-3cm. Specific application dates are presented in Table 1. The Btk was applied as Foray $48 \mathrm{~F}$ at a dosage rate of 40 Billion International Units (BIU)/acre. The GC was applied at a rate of $2 \times 10^{11}$ Polyhedral Inclusion Bodies (PIB)/acre. Applications to GW plots were accomplished by the use of helicopters, and the MON plots were sprayed utilizing fixed-wing aircraft.

Table 1. Treatment Application Dates.

\begin{tabular}{|c|c|c|c|c|c|c|c|c|}
\hline & \multicolumn{4}{|c|}{ George Washington National Forest } & \multicolumn{4}{|c|}{ Monongahela National Forest } \\
\hline & \multicolumn{2}{|c|}{ Btk } & \multicolumn{2}{|c|}{ Gypchek $^{\mathrm{TM}}$} & \multicolumn{2}{|c|}{ Btk } & \multicolumn{2}{|c|}{ Gypchek $^{\mathrm{TM}}$} \\
\hline Year & Plot & Treated & Plot & Treated & Plot & Treated & Plot & Treated \\
\hline 1997 & $\begin{array}{l}3 \\
5,7\end{array}$ & $\begin{array}{l}\text { May } 17 \\
\text { May } 18\end{array}$ & $\begin{array}{l}9 \\
1,6\end{array}$ & $\begin{array}{l}\text { May } 21 \\
\text { May } 23\end{array}$ & $\begin{array}{l}18 \\
11,15\end{array}$ & $\begin{array}{l}\text { May } 28 \\
\text { May } 29\end{array}$ & $10,13,17$ & May 23 \\
\hline 1998 & $3,5,7$ & May 10 & $1,6,9$ & May 07,08 & $11,15,18$ & May 15 & $10,13,17$ & May 13,14 \\
\hline
\end{tabular}

\section{Biological Insecticide Analysis}

To determine toxin concentrations on the foliage immediately following $B t$ application, samples were collected from each treatment plot within 2 hours of application. A total of 50 samples was collected along two transects within 20 ha core sub-plots established near the center of each of the $6 \mathrm{Bt}$ plots during 1997. Eight samples each were collected from plots 3, 5, 7, 11, and 15; Ten samples were collected from plot 18. In addition, 13 samples were collected from locations outside the core sub-plots (still within total plot boundaries) from designated foliage pruning areas. Four samples each were collected from plots 3 
and 5; Five samples were collected from plot 7. Samples consisted of leaf clusters which were clipped from the lower and mid-canopy, using pole pruners, from a height of approximately 2-3m. Several oak, maple, and hickory tree species were sampled. Leaves were individually placed in plastic, zipper sealed bags and placed in iced coolers for transport to the laboratory. Leaves were subsequently scanned with a leaf area meter, pooling leaves from individual samples. During 1998, 12 samples were collected from pruning areas (two from each $B t$ plot) and 24 samples were collected along the transects (four from each $B t$ plot). Though analyses of GC spray coverage were not specifically conducted, adequate coverage was indicated with the use of spray cards in the field.

For $B t$ analysis, toxins were extracted and concentrations of toxin $/ \mathrm{cm}^{2}$ leaf area were determined utilizing ELISA techniques. The ELISA technique is extremely sensitive to Btk protein toxins (Wie et al. 1982). Specifically, the Abbott Laboratories, Research Kit (DAS ELISA) for Btk endotoxin proteins was utilized. Following extraction of toxins, samples were frozen. The endotoxin analyses were conducted at the USDA-Forest Service Center for Forest Health Research Laboratories at Hamden, Connecticut, under the supervision of the late Dr. Norman Dubois, Research Microbiologist. Upon sample preparation, an automated plate reader was utilized to measure optical densities from which toxin concentrations were calculated.

\section{Lepidopteran Sampling}

\section{A. Adults}

Within the 20 ha core sub-plot of each of the 18 study plots, a 10-watt black light trap (BioQuip Products, Gardena, CA) was hung at a height of approximately $1.5 \mathrm{~m}$. The traps were utilized for the purpose of capturing light-attracted macrolepidopteran adults (Butler and Kondo 1991). Traps on all 18 plots were operated once during the same night each week for 15 weeks during the study. The regular 15 week

sampling seasons were initiated on 8 May 1995, 6 May 1996, 5 May 1997, and 11 May 1998. All samples were returned to the laboratory in chilled coolers and frozen prior to identification.

Nineteen species were selected from five families for individual wing length measurements and abundance determinations (see Table 2). These particular species were selected for more critical analysis due to their relatively high abundances determined during the first year of the study, and because it was 
suspected that larvae would likewise be abundant and easily collected from foliage and canvas bands. The premise for choosing more abundant species was that they have the potential to play a more pivotal role in the food chain. If such individuals were significantly affected by pesticide applications (lethally and/or sublethally), additional repercussions to the ecological system might result.

Species from several families were chosen to provide a larger cross-section of macrolepidopterans. Though the larvae and adults are present at different times during the sampling season, most of larvae of these particular species (like the gypsy moth) commonly feed on oak, maple, and hickory. It was expected that most species would not be affected by treatment applications mainly due to the fact that spraying was purposefully applied during gypsy moth larval susceptibility. Many of the other larvae appear later in the season. Furthermore, Btk used in this study is a strain specifically formulated for its capacity to target gypsy moths. Gypchek ${ }^{\mathrm{TM}}$ is highly specific for gypsy moth and would not be expected to be detrimental to other lepidopterans. A brief summary of the basic life histories of the 19 species selected for this study has been provided as Appendix I. 
Table 2. Selected Lepidopteran Families and Species.

\begin{tabular}{|l|l|}
\hline Geometridae: & Arctiidae: \\
Melanolophia canadaria $(\mathrm{Gn})$. & Hypoprepia fucosa $(\mathrm{Hbn})$. \\
Hypagyrtis unipunctata $(\mathrm{Hbn})$. & Halysidota tesselaris $(\mathrm{J} . \mathrm{E}$. Smith) \\
Lambdina fervidaria $(\mathrm{Hbn})$. & \\
Campaea perlata $(\mathrm{Gn})$. & Noctuidae: \\
Tetracis cachexiata $(\mathrm{Gn})$. & Achatia distincta $(\mathrm{Hbn})$. \\
Itame pustularia $(\mathrm{Gn})$. & Polia latex $(\mathrm{Gn})$. \\
Besma quercivoraria $(\mathrm{Gn})$. & Morrisonia confusa $(\mathrm{Hbn})$. \\
Besma endropiaria $(\mathrm{Gn})$. & Allotria elonympha $(\mathrm{Hbn})$. \\
& Baileya opthalmica $(\mathrm{Gn})$. \\
Lasiocampidae: & Acronicta ovata $(\mathrm{Grt})$. \\
Malacosoma disstria $(\mathrm{Hbn})$. & \\
Notodontidae: & \\
Heterocampa guttivitta $(\mathrm{Wlk})$. & \\
Nadata gibbosa $(\mathrm{J} . \mathrm{E}$. Smith) & \\
& \\
\hline
\end{tabular}

Light trap catches were individually placed into sorting trays and the 19 species of lepidopteran adults previously indicated were counted and separated by both species and sex for the 1995 (pretreatment) and 1998 (treatment) years. Total length (from wing angle to tip) of one of the front wings of each intact moth was then measured utilizing a digital caliper which was interfaced to a personal computer. TAL Technologies Software Wedge ${ }^{\mathrm{TM}}$, purchased through Forestry Suppliers, Inc., Jackson, Mississippi, was used for the interface. Due to the large number of moths collected from many catches, a limited amount of subsampling for wing measurements was conducted. All moths were sexed and all intact females were measured. When males of a particular species were sufficiently numerous in a sample, a minimum of 30 individuals (typically at least 100) was measured. Moths collected during the 1996 and 1997 sampling years were not sexed and measured; however, weekly totals of each species were used for abundance comparisons. 


\section{B. Larval Collection Techniques}

Larval macrolepidoptera were collected during 1995-1998 sampling seasons from all plots by two methods, canvas bands and foliage pruning. Canvas bands stapled to trees allowed for the capture of sheltering macrolepidopteran larvae which aggregated under the bands when traveling up tree trunks or down from the canopy (Cameron and Reeves 1990; Butler and Kondo 1993). Bands were attached to 12 trees within each sub-plot. An attempt was made to select trees (10 oaks, 1 hickory, and 1 maple) such that half of the trees was located at lower elevations within the sub-plot and the other half was located in upper elevations of the sub-plot. Bands were monitored once each week during each 15 week sampling season. Larval lepidopterans were placed in plastic vials which were subsequently chilled in a cooler prior to returning them to the laboratory for identification and rearing.

Macrolepidopteran larvae were also collected from foliage which was pruned weekly from each plot during each 15 week sampling season. Sampling occurred at two to three rotating sites located outside the core sub-plots. Pole pruners were utilized to collect samples from the lower and mid canopy (approximately 2-3 m) of mixed oaks, hickories, and maples (Cooper and Whitmore 1990; Butler 1992; Butler and Kondo 1993). Canopy sampling was initiated each season following bud-break and continued through the $15^{\text {th }}$ week of the sampling season. Each week, three oak, one maple, and one hickory sample were collected from each plot. Each sample consisted of 21 branch tips clipped from numerous trees of the same type. A hickory sample consisted of 15 branch tips. Samples were collected into large durable plastic bags and tied with a wire tie to be returned to the laboratory for gleaning of macrolepidopteran larvae. During the eighth week of the 1997 sampling season and all of the 1998 sampling season, larvae greater than $1^{\text {st }}$ instar (of the same selected species as the adults previously indicated) were weighed using a balance accurate to $0.0001 \mathrm{~g}$. 


\section{Larval Rearing Techniques}

After identification, most macrolepidopteran larvae older than $1^{\text {st }}$ instars were placed into $3.25 \mathrm{oz}$ individual plastic rearing cups with untreated foliage. Because foliage type may influence larval growth (Casagrande et al. 1987; Roth et al. 1997), effort was made to feed larvae clean foliage from the same tree genus from which it was collected. Larvae which were removed from canvas bands were fed foliage from the same genus of tree. In the case of Hypoprepia fucosa, which is known to feed on mosses and lichens, lichens were supplied. Fresh foliage was replaced on alternate days until the larva died, pupated, or upon the emergence of parasitoid(s). For the 19 species of larvae which were weighed, those which successfully pupated were weighed a second time within a few days after tanning.

\section{Results:}

\section{Bt Analysis}

Individual concentrations of $B t k$ toxins analyzed from collected leaf samples are presented in

Table 3. Site locations are identified with $\mathrm{P}$ or $\mathrm{T}$ referring to pruning areas or transect areas, respectively. Point locations indicate different samples for pruning areas and different locations along the transects which were previously established in the core plots. Concentrations varied between non-detectable levels and 171

$\mathrm{ng} / \mathrm{cm}^{2}$. Only 11 samples $\left(9.9 \%\right.$ ) were less than $20 \mathrm{ng} / \mathrm{cm}^{2}$ Above this level, approximately $100 \%$ of gypsy moths would be expected to receive a lethal dose (Dubois 1998 -unpublished experimental data). 
Table 3. Btk Toxin Concentrations on each sample.

\begin{tabular}{|c|c|c|c|c|c|c|c|c|c|}
\hline Plot & Site & Point & $1997(\mathrm{ng} / \mathrm{cm} 2)$ & $1998(\mathrm{ng} / \mathrm{cm} 2)$ & Plot & Site & Point & $1997(\mathrm{ng} / \mathrm{cm} 2)$ & $1998(\mathrm{ng} / \mathrm{cm} 2)$ \\
\hline \multirow[t]{12}{*}{3} & $\mathrm{P}$ & $.1 \mathrm{~m}$ & 36 & & 11 & $\mathrm{P}$ & 1 & 38 & \\
\hline & $\mathrm{P}$ & $.25 \mathrm{~m}$ & 27 & 38 & & $\mathrm{P}$ & 2 & 42 & 35 \\
\hline & $\mathrm{P}$ & $.35 \mathrm{~m}$ & 32 & 54 & & $\mathrm{P}$ & 3 & 100 & 52 \\
\hline & $\mathrm{P}$ & $.45 \mathrm{~m}$ & 27 & & & $\mathrm{P}$ & 4 & 50 & \\
\hline & $T$ & $\mathrm{~A} 00$ & 57 & & & $\mathrm{~T}$ & $\mathrm{~A} 00$ & 38 & \\
\hline & $T$ & A200 & 39 & 28 & & $\mathrm{~T}$ & A200 & 74 & 37 \\
\hline & $\mathrm{T}$ & A400 & 50 & 56 & & $\mathrm{~T}$ & A400 & 79 & 42 \\
\hline & $\mathrm{T}$ & A600 & 26 & & & $\mathrm{~T}$ & A600 & 65 & \\
\hline & $\mathrm{T}$ & D00 & 28 & & & $\mathrm{~T}$ & D00 & 101 & \\
\hline & $\mathrm{T}$ & D200 & 120 & 69 & & $\mathrm{~T}$ & D200 & 96 & 70 \\
\hline & $T$ & D400 & 22 & 15 & & $\mathrm{~T}$ & D400 & 78 & 32 \\
\hline & $\mathrm{T}$ & D600 & 75 & & & $\mathrm{~T}$ & $\mathrm{D} 600$ & 109 & \\
\hline Plot & Site & Point & $1997(\mathrm{ng} / \mathrm{cm} 2)$ & $1998(\mathrm{ng} / \mathrm{cm} 2)$ & Plot & Site & Point & $1997(\mathrm{ng} / \mathrm{cm} 2)$ & $1998(\mathrm{ng} / \mathrm{cm} 2)$ \\
\hline \multirow[t]{12}{*}{5} & $\mathrm{P}$ & 1 & 0 & & 15 & $\mathrm{P}$ & 1 & 40 & \\
\hline & $\mathrm{P}$ & 2 & 51 & 75 & & $\mathrm{P}$ & 2 & 39 & 67 \\
\hline & $\mathrm{P}$ & 3 & 160 & 80 & & $\mathrm{P}$ & 3 & 40 & 45 \\
\hline & $\mathrm{P}$ & 4 & 69 & & & $\mathrm{P}$ & 4 & 43 & \\
\hline & $\mathrm{T}$ & $\mathrm{A} 00$ & 57 & & & $\mathrm{~T}$ & $\mathrm{~A} 00$ & 44 & \\
\hline & $\mathrm{T}$ & A200 & 20 & 30 & & $\mathrm{~T}$ & A200 & 54 & 48 \\
\hline & $T$ & A400 & 46 & 32 & & $\mathrm{~T}$ & A400 & 89 & 36 \\
\hline & $\mathrm{T}$ & A600 & 17 & & & $\mathrm{~T}$ & A600 & 117 & \\
\hline & $T$ & D00 & 74 & & & $\mathrm{~T}$ & D00 & 81 & \\
\hline & $\mathrm{T}$ & D200 & 27 & 34 & & $\mathrm{~T}$ & D200 & 59 & 71 \\
\hline & $\mathrm{T}$ & D400 & 93 & 75 & & $\mathrm{~T}$ & D400 & 51 & 91 \\
\hline & $\mathrm{T}$ & D600 & 106 & & & $\mathrm{~T}$ & $\mathrm{D} 600$ & 96 & \\
\hline Plot & Site & Point & $1997(\mathrm{ng} / \mathrm{cm} 2)$ & $1998(\mathrm{ng} / \mathrm{cm} 2)$ & Plot & Site & Point & 1997 (ng/cm2) & $1998(\mathrm{ng} / \mathrm{cm} 2)$ \\
\hline \multirow[t]{14}{*}{7} & $\mathrm{P}$ & 1 & 138 & & 18 & $\mathrm{P}$ & 1 & 0 & \\
\hline & $\mathrm{P}$ & 2 & 150 & & & $\mathrm{P}$ & 2 & 6 & 13 \\
\hline & $\mathrm{P}$ & 3.1 & 171 & 53 & & $\mathrm{P}$ & 3 & 66 & 48 \\
\hline & $P$ & 3.2 & 77 & 39 & & $P$ & 4 & 27 & \\
\hline & $\mathrm{T}$ & 4 & 93 & & & $\mathrm{~T}$ & $\mathrm{~A} 00$ & 39 & \\
\hline & $T$ & $\mathrm{~A} 00$ & 97 & & & $\mathrm{~T}$ & A200 & 21 & 62 \\
\hline & $\mathrm{T}$ & A200 & 150 & 9 & & $\mathrm{~T}$ & A200R & 13 & 39 \\
\hline & $\mathrm{T}$ & A400 & 146 & 14 & & $\mathrm{~T}$ & A400 & 50 & \\
\hline & $T$ & A600 & 99 & & & $\mathrm{~T}$ & A600 & 8 & \\
\hline & $\mathrm{T}$ & D00 & 120 & & & $\mathrm{~T}$ & D00 & 38 & \\
\hline & $\mathrm{T}$ & D200 & 43 & 22 & & $\mathrm{~T}$ & D200 & 18 & 20 \\
\hline & $\mathrm{T}$ & D400 & 20 & 30 & & $\mathrm{~T}$ & D200R & 50 & \\
\hline & $\mathrm{T}$ & D600 & 140 & & & $\mathrm{~T}$ & D400 & 33 & 42 \\
\hline & & & & & & $\mathrm{T}$ & D600 & 35 & \\
\hline
\end{tabular}

Three sets of histograms illustrating the concentrations of toxins in relation to the two treatment years, the two forests, and the 6 individual treatment plots are presented as Figure 1. The first set of histograms illustrates that though most all of the concentrations fall within an effective dosage range, 1997 concentrations were significantly higher (Student's t-test, $\mathrm{p}<.05)$ on average than 1998 concentrations (63.1 and $44.5 \mathrm{ng} / \mathrm{cm}^{2}$ respectively). A 2-way analysis of variance (ANOVA) testing the main effects of YEAR 
and FOREST likewise demonstrated a significant difference between years $(\mathrm{P}=0.011)$, though differences in the FOREST main effect and in the interaction between YEAR x FOREST were not significant. The remaining two sets of histograms illustrate toxin concentrations of samples collected from the individual treatment plots on each forest. Again, analysis indicates that almost all of the samples contained significant levels of $B t$ toxins. 

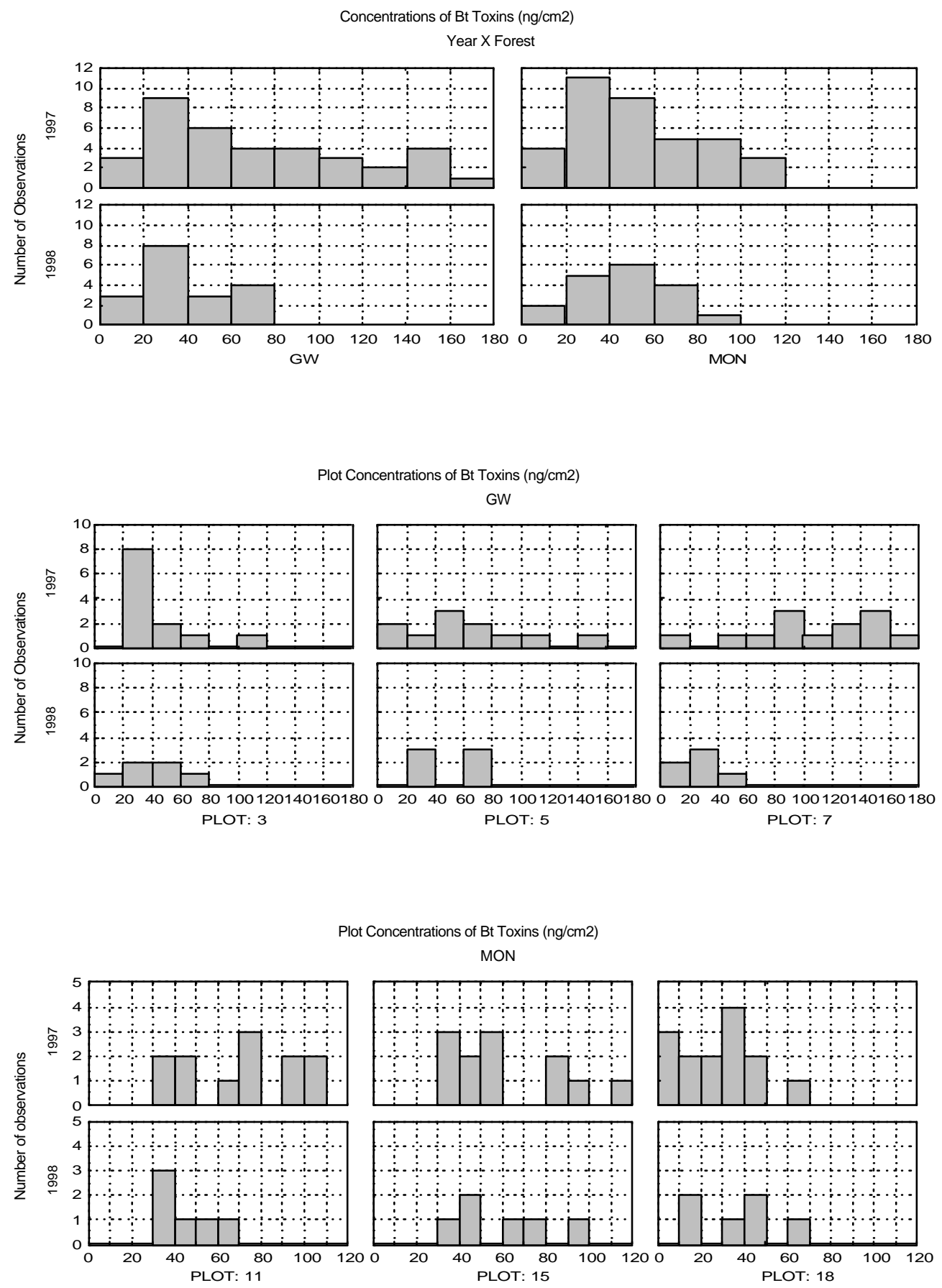

Figure 1. Histograms of Btk toxin concentrations. The first histogram illustrates the concentrations of Btk toxins from leaf samples collected during the 1997 and 1998 treatment years from the George Washington (GW) and Monongahela (MON) National Forests. The second two histograms show individual plot concentrations for each year from each forest. 


\section{Average Weekly Sampling Event Temperatures}

Average temperatures from maximum-minimum thermometers located at each plot on both forests were recorded during each weekly light-trap sampling event. Averages obtained from data collected during 1995-1998 are illustrated in three sets of graphs presented as Figure 2. The first set of graphs compares the average sampling event temperatures collected each week from each forest during the 1995 \& 1996 pretreatment years and the $1997 \& 1998$ treatment years. An ANOVA indicates significant differences between forests $(\mathrm{P}<0.05)$, but not between years. Average temperatures during pre-treatment years were 67.7 and $63.6^{\circ} \mathrm{F}$ for the GW and MON forests, respectively. During treatment years, averages were 67.7 and $67.7^{\circ} \mathrm{F}$ for the $\mathrm{GW}$ compared with the MON averages of 63.5 and $63.7^{\circ} \mathrm{F}$. The second set of graphs illustrate comparable temperatures among designated treatment assignments for the GW plots, while the third set of graphs illustrate comparable temperatures among designated treatment assignments for the MON plots. The third set of graphs also suggest that the first few weeks of the 1995 \& 1996 pretreatment years were cooler than the 1997 \& 1998 treatment years on the MON. A Student's t-test conducted on the first 5 weeks indicates the difference $\left(2.3^{\circ} \mathrm{F}\right)$ to be significant $(\mathrm{P}=0.035)$. 
Average Temperatures

GW \& MON
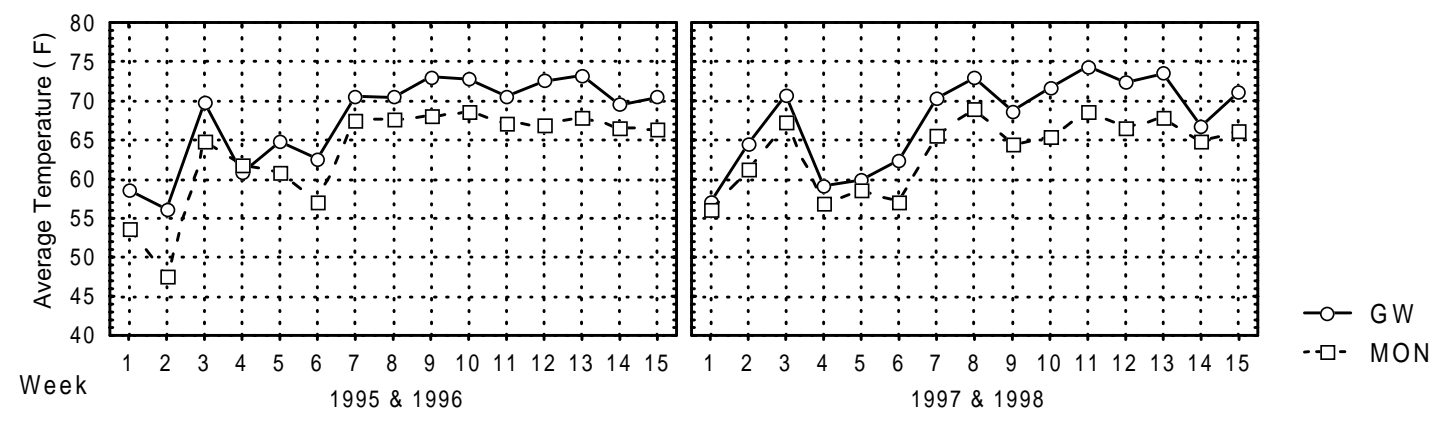

Average Temperatures Among Treatments

GW
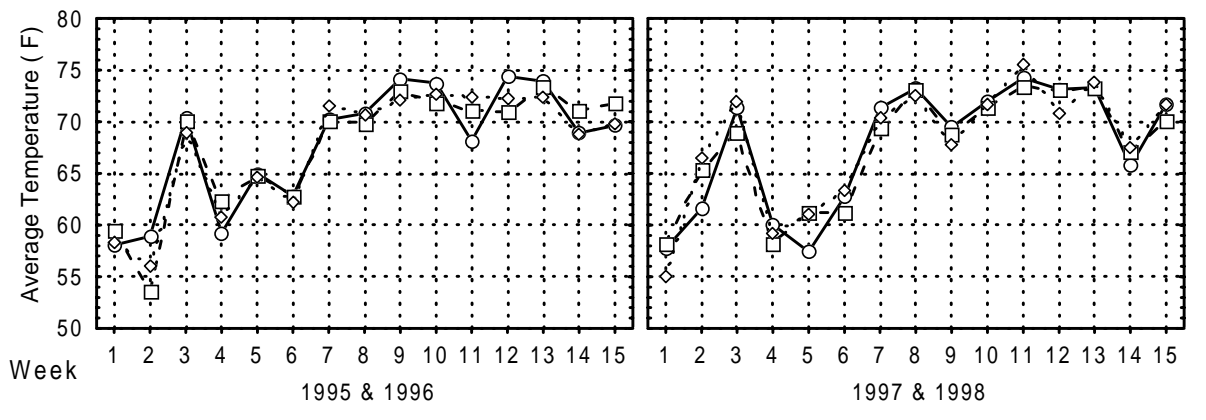

$-\circ-$ Reference
$-\square-\mathrm{Bt}$
.$\diamond \cdots$ Gypchek

Average Temperatures Among Treatments

MON
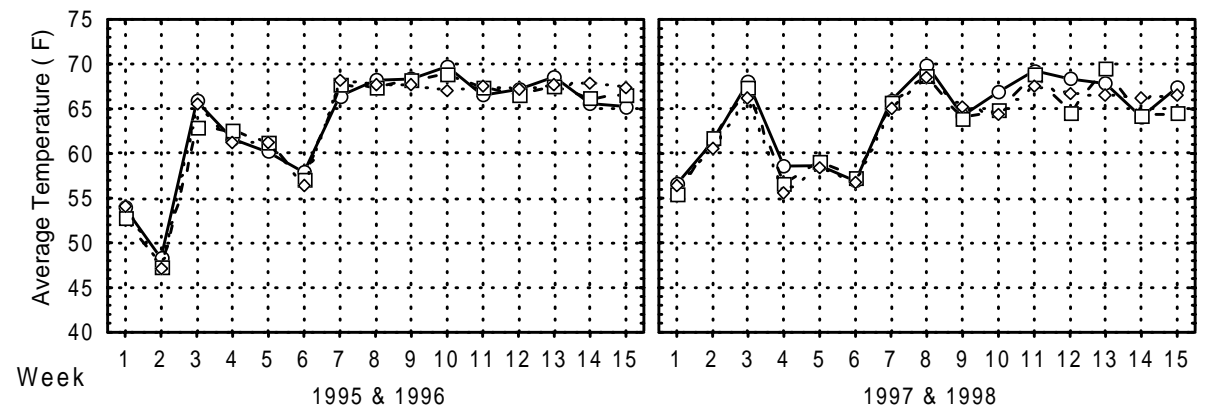

- - Reference

$-\square-B t$

$\cdots \diamond \cdots$ Gypchek

Figure 2. Average temperatures for weekly sampling events. The first graph illustrates average weekly temperatures during pretreatment and treatment years for the George Washington $(\mathrm{GW})$ and Monongahela (MON) National Forests. The second two graphs illustrate average weekly temperatures among treatment groups for each forest during pretreatment and treatment years. 


\section{Statistical Analyses Applied to Selected Lepidopteran Species:}

As in similar insect studies (i.e. Holloway 1984; Butler et al. 1997a,b), analysis of variance (ANOVA) was the statistical method utilized to analyze these data. Larvae collected from foliage and canvas bands were classified into pretreatment and treatment categories, thus combining years 1995 with 1996 (pretreatment), and years 1997 with 1998 (treatment). Adults collected from light traps were similarly grouped. In this case, a 3-way ANOVA was conducted utilizing these data. Main effects were YEARS (pretreatment vs. treatment), FOREST (GW vs. MON), and TREATMENT (Ref vs. $B t$ vs. GC ). PLOT was then nested within FOREST and TREATMENT and assigned as random for the ANOVA. Average COUNTS for each plot provided the dependent variable. Average larval count comparisons for these analyses, including positive interactions, are presented in Table 4. Differences which are significant at the $\mathrm{P}<0.05$ level are indicated by asterisks. Similarly, adult comparisons are presented in Table 5. Larval and adult species not collected in sufficient numbers to conduct the 3-way ANOVA were not included in these tables (i.e. several statistical categories had zero individuals).

Adults collected from years 1995 and 1998 were sexed and individual wing lengths were determined. For those species with sufficient numbers, SEX was added to provide for a 4-way ANOVA (see Table 6). As with the previous data, significant differences and interactions are indicated with asterisks. For those species where sufficient numbers of adults were intact enough to provide wing measurements, a 4-way ANOVA was conducted using WINGLENGTH as the response. Average wing length differences are presented in Table 7.

Often, males were collected in significantly higher numbers than females. Consequently, if females were collected in extremely low numbers, males were analyzed alone without SEX in the model. Male counts and average wing length comparisons from these analyses are provided as Table $\mathbf{8}$ and Table $\mathbf{9}$, respectively. Table 10 provides wing length comparisons between males and females of each species for which a simple 1way ANOVA was conducted.

Average weights obtained from larvae collected during 1997 and 1998 treatment years were compared utilizing a 2-way ANOVA with FOREST and TREATMENT as main effects and PLOT nested within FOREST and TREATMENT. These comparisons are presented in Table 11. Due to the relatively small number of larvae collected, only 2 species were analyzed separately (Besma endropiaria (Gn.) and Polia latex (Gn.)). However, 
total larvae, geometrids, and noctuids were grouped together for additional comparisons. Likewise, pupal weights were grouped. Separate analysis was provided only for Polia latex (see Table 12).

Table 4. Average Larval Counts/plot (1995-1998)

\begin{tabular}{|c|c|c|c|c|}
\hline & All Species & Geometrids & Noctuids & Melanolophia canadaria \\
\hline \multicolumn{5}{|l|}{ Main Effects } \\
\hline Years (1995-1996 / 1997-1998) & $182.9 / 216.7^{\star *}$ & $44.7 / 51.2$ & $101.9 / 145.6^{\star *}$ & $11.7 / 5.9^{* *}$ \\
\hline Forest (GW / MON) & $161.1 / 238.5$ & $41.9 / 54.0$ & $90.9 / 156.6$ & $9.8 / 7.8$ \\
\hline Treatment (Ref / Bt / GC) & $215.6 / 180.8 / 203.0$ & $57.4 / 34.9 / 51.5$ & $124.9 / 118.4 / 127.9$ & $11.6 / 6.0 / 8.8$ \\
\hline \multicolumn{5}{|l|}{ Interaction } \\
\hline Year X Forest & ** & ** & NS & ** \\
\hline Year $\mathrm{X}$ Treatment & NS & NS & NS & NS \\
\hline \multirow[t]{2}{*}{ Year X Forest X Treatment } & NS & NS & NS & NS \\
\hline & Hypagyrtis unipunctata & Lambdina fervidaria & Campaea perlata & Tetracis cachexiata \\
\hline \multicolumn{5}{|l|}{ Main Effects } \\
\hline Years (1995-1996 / 1997-1998) & $5.3 / 6.9$ & $3.7 / 5.4$ & $4.6 / 5.2$ & $1.5 / 2.2$ \\
\hline Forest (GW / MON) & $5.3 / 6.9$ & $2.9 / 6.3^{\star *}$ & $6.2 / 3.6$ & $2.2 / 11.5$ \\
\hline Treatment (Ref / Bt / GC) & $6.8 / 4.4 / 7.2$ & $6.0 / 2.5 / 5.3$ & $6.8 / 3.3 / 4.6$ & $2.5 / 1.2 / 1.8$ \\
\hline \multicolumn{5}{|l|}{ Interaction } \\
\hline Year X Forest & ** & NS & NS & NS \\
\hline Year X Treatment & NS & ** & NS & NS \\
\hline \multirow[t]{2}{*}{ Year X Forest X Treatment } & NS & NS & NS & NS \\
\hline & Itame pustularia & Besma quercivoraria & Besma endropiaria & Heterocampa guttivitta \\
\hline \multicolumn{5}{|l|}{ Main Effects } \\
\hline Years (1995-1996 / 1997-1998) & $7.3 / 5.3$ & $7.3 / 3.7^{* \star}$ & $3.3 / 16.6^{\star *}$ & $6.3 / 10.3^{\star *}$ \\
\hline Forest (GW / MON) & $4.9 / 7.7$ & $4.3 / 6.7$ & $6.3 / 13.6^{\star *}$ & $5.8 / 10.7^{\star \star}$ \\
\hline Treatment (Ref / Bt / GC) & $6.6 / 4.5 / 7.8$ & $5.8 / 5.3 / 5.4$ & $11.3 / 7.8 / 10.7$ & $10.9 / 7.4 / 6.5$ \\
\hline \multicolumn{5}{|l|}{ Interaction } \\
\hline Year X Forest & ** & NS & ** & ** \\
\hline Year X Treatment & NS & NS & NS & ** \\
\hline \multirow[t]{2}{*}{ Year X Forest X Treatment } & NS & NS & NS & NS \\
\hline & Nadata gibbosa & Hypoprepia fucosa & Halysidota tesselaris & Achatia distincta \\
\hline \multicolumn{5}{|l|}{ Main Effects } \\
\hline Years (1995-1996 / 1997-1998) & $7.6 / 2.6^{\star \star}$ & $15.0 / 4.3^{\star \star}$ & $5.5 / 2.5^{\star \star}$ & $1.5 / 1.6$ \\
\hline Forest (GW / MON) & $6.2 / 3.9$ & $11.1 / 8.2$ & $4.6 / 3.4$ & $1.8 / 1.3$ \\
\hline Treatment (Ref / Bt / GC) & $5.6 / 4.0 / 5.7$ & $12.6 / 10.1 / 6.3$ & $3.6 / 3.9 / 4.5$ & $1.8 / 0.9 / 2.0$ \\
\hline \multicolumn{5}{|l|}{ Interaction } \\
\hline Year X Forest & NS & NS & ** & NS \\
\hline Year X Treatment & NS & NS & ** & ** \\
\hline \multirow[t]{2}{*}{ Year X Forest X Treatment } & NS & NS & NS & NS \\
\hline & Polia latex & Morrisonia confusa & Baileya opthalmica & Acronicta ovata \\
\hline \multicolumn{5}{|l|}{ Main Effects } \\
\hline Years (1995-1996 / 1997-1998) & $29.3 / 61.4^{\star \star}$ & $4.1 / 13.9^{* *}$ & $3.5 / 3.6$ & $63.6 / 64.6$ \\
\hline Forest (GW / MON) & $49.8 / 40.9$ & $4.9 / 13.1^{\star *}$ & $1.1 / 6.0^{* *}$ & $33.1 / 95.1^{\star *}$ \\
\hline Treatment (Ref / Bt / GC) & $45.5 / 42.7 / 47.8$ & $9.8 / 8.8 / 8.3$ & $2.3 / 4.5 / 3.8$ & $65.3 / 61.5 / 65.4$ \\
\hline \multicolumn{5}{|l|}{ Interaction } \\
\hline Year X Forest & NS & ** & ** & NS \\
\hline Year X Treatment & NS & NS & NS & NS \\
\hline Year X Forest X Treatment & NS & NS & ** & NS \\
\hline \multicolumn{5}{|l|}{${ }^{\star *}$ Indicates Significant } \\
\hline \multicolumn{5}{|l|}{ Differences $(p<.05)$} \\
\hline \multicolumn{5}{|l|}{ Non-Listed Interactions and } \\
\hline \multicolumn{5}{|l|}{ Interactions Signified with NS } \\
\hline Are Not Significant $(p>.05)$ & & & & \\
\hline
\end{tabular}


Table 5. Average Adult Counts/plot (1995-1998).

\begin{tabular}{|c|c|c|c|c|}
\hline & All Species & Geometrids & Noctuids & Melanolophia canadaria \\
\hline \multicolumn{5}{|l|}{ Main Effects } \\
\hline Years (1995-1996 / 1997-1998) & $2375 / 3407^{* \star}$ & $710 / 955$ & $875 / 1635^{\star \star}$ & $43 / 62$ \\
\hline Forest (GW / MON) & $2007 / 3776^{\star \star}$ & $501 / 1164^{\star \star}$ & $733 / 1778$ & $25 / 80^{\star *}$ \\
\hline Treatment (Ref / Bt / GC) & 3725 / 2364 / 2584 & $953 / 814 / 730$ & $1889 / 843 / 1034$ & $55 / 61 / 42$ \\
\hline \multicolumn{5}{|l|}{ Interaction } \\
\hline Year X Forest & ** & ** & NS & ** \\
\hline Year X Treatment & NS & NS & NS & NS \\
\hline \multirow[t]{2}{*}{ Forest X Treatment } & NS & NS & NS & NS \\
\hline & Hypagyrtis unipunctata & Lambdina fervidaria & Campaea perlata & Tetracis cachexiata \\
\hline \multicolumn{5}{|l|}{ Main Effects } \\
\hline Years (1995-1996 / 1997-1998) & $92 / 66^{\star \star}$ & $184 / 428^{\star \star}$ & $40 / 96^{\star \star}$ & $21 / 76^{\star \star}$ \\
\hline Forest (GW / MON) & $67 / 90$ & $91 / 521^{\star *}$ & $43 / 93$ & $22 / 75^{\star \star}$ \\
\hline Treatment (Ref / Bt / GC) & $65 / 75 / 97$ & $400 / 262 / 256$ & $74 / 79 / 51$ & $57 / 46 / 43$ \\
\hline \multicolumn{5}{|l|}{ Interaction } \\
\hline Year X Forest & NS & ** & NS & NS \\
\hline Year X Treatment & ** & NS & NS & NS \\
\hline \multirow[t]{2}{*}{ Forest X Treatment } & NS & NS & NS & NS \\
\hline & Itami pustularia & Besma quercivoraria & Besma endropiaria & Malacosoma disstria \\
\hline \multicolumn{5}{|l|}{ Main Effects } \\
\hline Years (1995-1996 / 1997-1998) & $224 / 117$ & $56 / 35^{\star *}$ & $51 / 74$ & $36 / 45$ \\
\hline Forest (GW / MON) & $189 / 152$ & $42 / 49$ & $21 / 104^{\star \star}$ & $53 / 28$ \\
\hline Treatment (Ref / Bt / GC) & $188 / 182 / 142$ & $40 / 54 / 42$ & $75 / 56 / 57$ & $42 / 22 / 57$ \\
\hline \multicolumn{5}{|l|}{ Interaction } \\
\hline Year X Forest & ** & ** & NS & NS \\
\hline Year X Treatment & NS & NS & NS & ** \\
\hline \multirow[t]{2}{*}{ Forest X Treatment } & NS & NS & NS & NS \\
\hline & Heterocampa quttivitta & Nadata qibbosa & Hypoprepia fucosa & Halysidota tesselaris \\
\hline \multicolumn{5}{|l|}{ Main Effects } \\
\hline Years (1995-1996 / 1997-1998) & $70 / 170^{\star *}$ & $99 / 75^{\star \star}$ & $409 / 288^{\star \star}$ & $175 / 240^{\text {** }}$ \\
\hline Forest (GW / MON) & $89 / 150^{\star \star}$ & $95 / 80$ & $339 / 357$ & $198 / 217$ \\
\hline Treatment (Ref / Bt / GC) & $126 / 101 / 133$ & $96 / 84 / 81$ & $365 / 317 / 363$ & $254 / 183 / 186$ \\
\hline \multicolumn{5}{|l|}{ Interaction } \\
\hline Year X Forest & ** & NS & NS & ** \\
\hline Year X Treatment & NS & NS & NS & NS \\
\hline \multirow[t]{2}{*}{ Forest X Treatment } & NS & NS & NS & NS \\
\hline & Achatia distincta & Polia latex & Morrisonia confusa & Allotria elonympha \\
\hline \multicolumn{5}{|l|}{ Main Effects } \\
\hline Years (1995-1996 / 1997-1998) & $4 / 4$ & $25 / 81^{\star *}$ & $27 / 70^{\star *}$ & $18 / 38^{* *}$ \\
\hline Forest (GW / MON) & $4 / 4$ & $39 / 66^{\star *}$ & $33 / 63^{* *}$ & $54 / 3^{\star \star}$ \\
\hline Treatment (Ref / Bt / GC) & $5 / 5 / 3$ & $69 / 42 / 48$ & $59 / 44 / 42$ & $23 / 30 / 31$ \\
\hline \multicolumn{5}{|l|}{ Interaction } \\
\hline Year X Forest & NS & NS & NS & ** \\
\hline Year X Treatment & NS & NS & NS & NS \\
\hline \multirow[t]{2}{*}{ Forest X Treatment } & NS & NS & $\star \star *$ & NS \\
\hline & Baileya opthalmica & Acronicta ovata & & \\
\hline \multicolumn{5}{|l|}{ Main Effects } \\
\hline Years (1995-1996 / 1997-1998) & $24 / 49^{* *}$ & $777 / 1394^{* *}$ & & \\
\hline Forest (GW / MON) & $14 / 159^{\star *}$ & $589 / 1582$ & & \\
\hline Treatment (Ref / Bt / GC) & $39 / 35 / 35$ & $1695 / 688 / 875$ & & \\
\hline \multicolumn{5}{|l|}{ Interaction } \\
\hline Year X Forest & ** & NS & & \\
\hline Year X Treatment & NS & NS & & \\
\hline Forest $\mathrm{X}$ Treatment & NS & NS & & \\
\hline & & & & \\
\hline${ }^{\star *}$ Indicates Significant & & & & \\
\hline Differences $(p<.05)$ & & & & \\
\hline Non-Listed Interactions and & & & & \\
\hline Interactions Signified with NS & & & & \\
\hline
\end{tabular}


Table 6. Average Adult Counts/plot (1995 \& 1998)

\begin{tabular}{|c|c|c|c|c|c|}
\hline & All Species & Geometrids & Noctuids & Melanolophia canadaria & Hypagyrtis unipunctata \\
\hline \multicolumn{6}{|l|}{ Main Effects } \\
\hline Sex (Female / Male) & $273.1 / 1063.3^{* \star}$ & $129.0 / 349.9^{* *}$ & $122.1 / 340.6^{\star \star}$ & $5.4 / 24.7^{\star \star}$ & $2.1 / 29.4^{\star \star}$ \\
\hline Year (1995 / 1998) & $569.8 / 766.5$ & $190.6 / 288.3$ & $150.1 / 312.7^{* *}$ & $10.7 / 19.4$ & $18.0 / 13.4^{* *}$ \\
\hline Forest (GW / MON) & $486.9 / 849.4^{\star \star}$ & $136.2 / 342.7^{\star *}$ & $163.0 / 299.8$ & $6.3 / 23.7^{\star \star}$ & $17.0 / 14.4$ \\
\hline Treatment (Ref / Bt / GC) & 823.4 / $558.9 / 622.3$ & $300.8 / 216.5 / 201.0$ & $298.7 / 173.2 / 222.3$ & $15.9 / 17.8 / 11.4$ & $11.6 / 14.7 / 20.8$ \\
\hline \multicolumn{6}{|l|}{ Interactions } \\
\hline Sex X Year & NS & NS & NS & ** & NS \\
\hline Sex X Forest & NS & NS & NS & ** & NS \\
\hline Year X Forest & ** & ** & NS & ** & NS \\
\hline Sex X Year X Forest & NS & NS & NS & ** & $\star *$ \\
\hline Sex X Year X Treatment & NS & NS & NS & NS & $\star \star *$ \\
\hline \multirow[t]{3}{*}{ Year X Forest $\mathrm{X}$ Teatment } & NS & NS & NS & NS & NS \\
\hline & & & & & \\
\hline & Lambdina fervidaria & Campaea perlata & Tetracis cachexiata & Itame pustularia & Besma quercivoraria \\
\hline \multicolumn{6}{|l|}{ Main Effects } \\
\hline Sex (Female / Male) & $51.4 / 103.3^{* *}$ & $4.9 / 40.3^{\star \star}$ & $2.9 / 25.7^{\star \star}$ & $47.7 / 84.3^{* *}$ & $2.5 / 16.4^{\star *}$ \\
\hline Year (1995 / 1998) & $32.6 / 122.1^{* *}$ & $12.5 / 32.7$ & $5.6 / 23.0^{\star *}$ & $81.3 / 50.7$ & $14.9 / 3.9^{\star *}$ \\
\hline Forest (GW / MON) & $17.2 / 137.4^{\star \star}$ & $12.3 / 32.9$ & $5.8 / 22.8^{\star *}$ & $66.8 / 65.2$ & $7.1 / 11.7$ \\
\hline Treatment (Ref / Bt / GC) & $116.3 / 53.9 / 61.8$ & $27.3 / 24.1 / 16.8$ & $18.7 / 11.3 / 12.9$ & $77.7 / 66.2 / 54.1$ & $7.8 / 12.9 / 7.6$ \\
\hline \multicolumn{6}{|l|}{ Interactions } \\
\hline Sex X Year & NS & ** & ** & NS & ** \\
\hline Sex X Forest & $\star *$ & ** & ** & $\star *$ & ** \\
\hline Year X Forest & ** & NS & NS & ** & ** \\
\hline Sex X Year X Forest & NS & NS & NS & NS & ** \\
\hline Sex X Year X Treatment & NS & NS & NS & NS & NS \\
\hline \multirow[t]{2}{*}{ Year X Forest X Teatment } & NS & NS & NS & NS & NS \\
\hline & Besma endropiaria & Malacosoma disstria & Heterocampa guttivitta & Nadata gibbosa & Hypoprepia fucosa \\
\hline \multicolumn{6}{|c|}{ 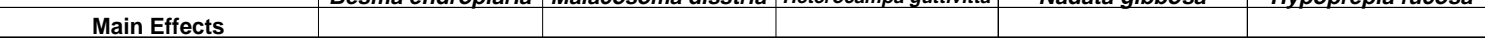 } \\
\hline Sex (Female / Male) & $12.3 / 26.0^{\star \star}$ & $0.6 / 23.0^{\star *}$ & $1.6 / 66.4^{\star *}$ & $2.5 / 44.8^{\star *}$ & $12.7 / 17.43^{\star *}$ \\
\hline Year (1995 / 1998) & $15.1 / 23.0$ & $9.4 / 14.2$ & $16.4 / 51.6^{\star *}$ & $32.9 / 14.5^{\star \star}$ & $138.3 / 48.7^{* *}$ \\
\hline Forest (GW / MON) & $3.6 / 34.6^{\star *}$ & $18.1 / 5.5^{\star \star}$ & $22.6 / 44.4$ & $27.9 / 19.4$ & $84.1 / 102.8$ \\
\hline Treatment (Ref / Bt / GC) & $25.6 / 15.8 / 16.1$ & $13.4 / 4.9 / 17.2$ & $40.1 / 26.9 / 35.0$ & $24.7 / 23.8 / 22.4$ & $106.6 / 78.8 / 95.0$ \\
\hline \multicolumn{6}{|l|}{ Interactions } \\
\hline Sex X Year & NS & NS & ** & ** & ** \\
\hline Sex X Forest & ** & ** & NS & NS & NS \\
\hline Year X Forest & NS & ** & NS & NS & NS \\
\hline & Halysidota tesselaris & Polia latex & Morrisonia confusa & Baileya opthalmica & Acronicta ovata \\
\hline \multicolumn{6}{|l|}{ Main Effects } \\
\hline Sex (Female / Male) & $4.5 / 64.2^{\star *}$ & $11.6 / 18.4^{* *}$ & $15.6 / 20.9$ & $8.2 / 13.5^{\star \star}$ & $83.0 / 276.4^{* *}$ \\
\hline Year (1995 / 1998) & $32.2 / 36.5$ & $5.2 / 24.8^{\star *}$ & $7.9 / 27.7^{\star *}$ & $5.3 / 16.4^{\star *}$ & $126.6 / 232.9$ \\
\hline Forest (GW / MON) & $33.9 / 34.8$ & $12.3 / 17.6$ & $9.3 / 26.3^{\star \star}$ & $3.8 / 17.9^{\star \star}$ & $122.7 / 236.8$ \\
\hline Treatment (Ref / Bt / GC) & $39.0 / 34.7 / 29.3$ & $21.7 / 10.5 / 12.7$ & $23.0 / 15.6 / 14.8$ & $13.1 / 9.1 / 10.3$ & $233.2 / 129.7 / 176.3$ \\
\hline \multicolumn{6}{|l|}{ Interactions } \\
\hline Sex X Year & NS & NS & NS & ** & NS \\
\hline Sex X Forest & NS & NS & NS & ** & NS \\
\hline Year X Forest & NS & NS & NS & ** & NS \\
\hline Sex X Treatment & NS & NS & NS & NS & NS \\
\hline Forest $\mathrm{X}$ Treatment & NS & NS & ** & NS & NS \\
\hline Sex X Year X Forest & NS & NS & NS & ** & NS \\
\hline Sex X Year X Treatment & NS & NS & NS & ** & NS \\
\hline \multicolumn{6}{|l|}{${ }^{\star *}$ Indicates Significant } \\
\hline \multicolumn{6}{|l|}{ Differences $(p<.05)$} \\
\hline \multicolumn{6}{|l|}{ Non-Listed Interactions and } \\
\hline \multicolumn{6}{|l|}{ Interactions Signified with NS } \\
\hline Are Not Significant $(p>.05)$ & & & & & \\
\hline
\end{tabular}


Table 7. Average Wing Length/plot (mm)

\begin{tabular}{|c|c|c|c|c|}
\hline & All Species & Geometrids & Noctuids & Lambdina fervidaria \\
\hline \multicolumn{5}{|l|}{ Main Effects } \\
\hline Sex (Female / Male) & $17.460 / 17.647$ & $17.327 / 16.865$ & $17.424 / 16.788^{\star *}$ & $18.501 / 18.220^{\star *}$ \\
\hline Year (1995 / 1998) & $17.212 / 17.896^{\star *}$ & $16.214 / 17.977^{\star *}$ & $17.035 / 17.177$ & $18.002 / 18.719^{\star *}$ \\
\hline Forest (GW / MON) & $17.592 / 17.516$ & $16.841 / 17.350$ & $17.778 / 16.935^{\star \star}$ & $18.043 / 18.678^{\star \star}$ \\
\hline Treatment (Ref / Bt / GC) & $17.492 / 17.597 / 17.572$ & $16.914 / 17.267 / 17.107$ & $17.055 / 17.149 / 17.115$ & $18.292 / 18.446 / 18.344$ \\
\hline \multicolumn{5}{|l|}{ Interactions } \\
\hline Sex X Year & ** & ** & NS & NS \\
\hline Sex X Forest & ** & ** & ** & ** \\
\hline Year X Forest & NS & ** & ** & ** \\
\hline Sex X Year X Forest & NS & NS & ** & NS \\
\hline \multirow[t]{4}{*}{ Year X Forest X Teatment } & NS & NS & NS & ** \\
\hline & & & & \\
\hline & & & & \\
\hline & Campaea perlata & Tetracis cachexiata & Itame pustularia & Hypoprepia fucosa \\
\hline \multicolumn{5}{|l|}{ Main Effects } \\
\hline Sex (Female / Male) & $25.288 / 21.474^{* *}$ & $23.570 / 21.053^{\star *}$ & $13.203 / 12.288^{* *}$ & $16.386 / 15.014^{* *}$ \\
\hline Year (1995 / 1998) & $23.434 / 23.328$ & $22.175 / 22.448$ & $12.655 / 12.835^{\star *}$ & $15.722 / 15.678$ \\
\hline Forest (GW / MON) & $23.571 / 23.190$ & $22.634 / 21.989^{* *}$ & $12.673 / 12.817$ & $15.698 / 15.702$ \\
\hline Treatment (Ref / Bt / GC) & $23.140 / 23.481 / 23.521$ & $22.530 / 22.414 / 21.991$ & $12.797 / 12.694 / 12.745$ & $15.726 / 15.773 / 15.601$ \\
\hline \multicolumn{5}{|l|}{ Interactions } \\
\hline Sex X Year & NS & NS & ** & NS \\
\hline Sex X Forest & NS & NS & NS & NS \\
\hline Year X Forest & NS & NS & NS & ** \\
\hline \multirow[t]{2}{*}{ Sex X Year X Forest } & NS & NS & NS & NS \\
\hline & Halysidota tesselaris & Polia latex & Morrisonia confusa & Baileya opthlamica \\
\hline \multicolumn{5}{|l|}{ Main Effects } \\
\hline Sex (Female / Male) & $22.976 / 21.966^{\star *}$ & $21.155 / 19.837^{\star *}$ & $18.086 / 16.946^{\star *}$ & $15.189 / 14.852^{\star *}$ \\
\hline Year (1995 / 1998) & $22.516 / 22.425$ & $20.764 / 20.228^{\star *}$ & $17.838 / 17.195^{\star *}$ & $15.304 / 14.737^{\star *}$ \\
\hline Forest (GW / MON) & $22.622 / 22.320^{\star \star}$ & $20.847 / 20.145^{\star \star}$ & $17.636 / 17.396^{\star \star}$ & $14.992 / 15.049$ \\
\hline Treatment (Ref / Bt / GC) & $22.480 / 22.379 / 22.553$ & $20.348 / 20.621 / 20.519$ & $17.501 / 17.554 / 17.494$ & $14.942 / 15.031 / 15.088$ \\
\hline \multicolumn{5}{|l|}{ Interactions } \\
\hline \multirow[t]{2}{*}{ Sex X Year } & ** & NS & NS & NS \\
\hline & Acronicta ovata & & & \\
\hline \multicolumn{5}{|l|}{ Main Effects } \\
\hline Sex (Female / Male) & $16.960 / 16.478^{\star \star}$ & & & \\
\hline Year (1995 / 1998) & $16.746 / 16.692$ & & & \\
\hline Forest (GW / MON) & $16.794 / 16.644^{\star \star}$ & & & \\
\hline Treatment (Ref / Bt / GC) & $16.666 / 16.724 / 16.768$ & & & \\
\hline \multicolumn{5}{|l|}{ Interactions } \\
\hline Sex X Year & NS & & & \\
\hline \multicolumn{5}{|l|}{ ** Indicates Significant } \\
\hline \multicolumn{5}{|l|}{ Differences $(p<.05)$} \\
\hline \multicolumn{5}{|l|}{ Non-Listed Interactions and } \\
\hline \multicolumn{5}{|l|}{ Effects Signified with NS are } \\
\hline Not Significant $(p>.05)$ & & & & \\
\hline
\end{tabular}


Table 8. Average Male Counts/plot (1995 \& 1998).

\begin{tabular}{|c|c|c|c|}
\hline & Melanolophia canadaria & Hypagyrtis unipunctata & Besma quercivoraria \\
\hline \multicolumn{4}{|l|}{ Main Effects } \\
\hline Year (1995 / 1998) & $16.1 / 33.2$ & $32.8 / 25.8$ & $25.3 / 7.4^{\star \star}$ \\
\hline Forest (GW / MON) & $9.5 / 39.8^{* *}$ & $31.3 / 27.3$ & $11.2 / 21.6$ \\
\hline Treatment (Ref / Bt / GC) & $25.6 / 28.7 / 19.8$ & $21.9 / 27.5 / 38.5$ & $12.7 / 23.8 / 12.6$ \\
\hline \multicolumn{4}{|l|}{ Interaction } \\
\hline \multirow[t]{2}{*}{ Year X Forest } & ** & NS & ** \\
\hline & Heterocampa guttivitta & Nadata gibbosa & Polia latex \\
\hline \multicolumn{4}{|l|}{ Main Effects } \\
\hline Year (1995 / 1998) & $31.8 / 101.1^{* *}$ & $61.9 / 27.7^{\star *}$ & $7.1 / 29.7^{\star *}$ \\
\hline Forest (GW / MON) & $45.7 / 87.1$ & $51.7 / 37.9$ & $16.7 / 20.1$ \\
\hline Treatment (Ref / Bt / GC) & $78.8 / 52.3 / 68.2$ & $46.1 / 46.0 / 42.4$ & $28.8 / 11.7 / 14.6$ \\
\hline \multicolumn{4}{|l|}{ Interaction } \\
\hline Year X Forest & NS & NS & NS \\
\hline \multicolumn{4}{|l|}{ ** Indicates Significant } \\
\hline \multicolumn{4}{|l|}{ Differences $(p<.05)$} \\
\hline \multicolumn{4}{|l|}{ Non-Listed Interactions and } \\
\hline \multicolumn{4}{|l|}{ Interactions Signified with NS } \\
\hline Are Not Siqnificant $(p>.05)$ & & & \\
\hline
\end{tabular}

Table 9. Average Male Wing Lengths/plot (mm).

\begin{tabular}{|c|c|c|c|}
\hline & Melanolophia canadaria & Hypagyrtis unipunctata & Besma quercivoraria \\
\hline \multicolumn{4}{|l|}{ Main Effects } \\
\hline Year (1995 / 1998) & $18.019 / 18.618^{\star *}$ & $15.935 / 15.429^{* \star}$ & $16.981 / 16.896$ \\
\hline Forest (GW / MON) & $18.247 / 18.391$ & $15.711 / 15.654$ & $16.861 / 17.015$ \\
\hline Treatment (Ref / Bt / GC) & $18.319 / 18.398 / 18.239$ & $15.854 / 15.601 / 15.543$ & $16.846 / 17.030 / 16.939$ \\
\hline \multicolumn{4}{|l|}{ Interactions } \\
\hline Year X Forest & NS & NS & NS \\
\hline \multirow[t]{2}{*}{ Year X Forest X Treatment } & NS & ** & NS \\
\hline & Heterocamp guttivitta & Nadata gibbosa & Polia latex \\
\hline \multicolumn{4}{|l|}{ Main Effects } \\
\hline Year (1995 / 1998) & $20.611 / 20.500$ & 24.152 / 23.977 & $20.029 / 19.644$ \\
\hline Forest (GW / MON) & $20.327 / 20.785^{\star \star}$ & $24.107 / 24.022$ & $20.104 / 19.570^{* *}$ \\
\hline Treatment (Ref / Bt / GC) & $20.525 / 20.495 / 20.646$ & $24.041 / 24.179 / 23.973$ & $19.670 / 19.949 / 19.891$ \\
\hline \multicolumn{4}{|l|}{ Interactions } \\
\hline Year X Forest & ** & NS & NS \\
\hline Year X Forest X Treatment & NS & NS & NS \\
\hline \multicolumn{4}{|l|}{ ** Indicates Significant } \\
\hline \multicolumn{4}{|l|}{ Differences $(p<.05)$} \\
\hline \multicolumn{4}{|l|}{ Non-Listed Interactions and } \\
\hline \multicolumn{4}{|l|}{ Interactions Signified with NS } \\
\hline Are Not Significant $(p>.05)$ & & & \\
\hline
\end{tabular}


Table 10. Female and male adult counts and wing lengths (1995 \& 1998).

\begin{tabular}{|c|c|c|c|c|c|}
\hline & Female Count & $\begin{array}{c}\text { Average } \\
\text { Wing Length }(\mathrm{mm})\end{array}$ & Male Count & $\begin{array}{c}\text { Average } \\
\text { Wing Length }(\mathrm{mm})\end{array}$ & $\begin{array}{c}\text { 1-Way } \\
\text { ANOVA }\end{array}$ \\
\hline Melanolophia canadaria (Gn.) & 194 & 17.633 & 888 & 18.449 & $\mathrm{P}<0.001 *$ \\
\hline Hypagyrtis unipunctata (Hbn.) & 76 & 17.090 & 1,055 & 15.711 & $\mathrm{P}<0.001 *$ \\
\hline Lambdina fervidaria (Hbn.) & 1,849 & 18.574 & 3,718 & 18.645 & $\mathrm{P}=0.099$ \\
\hline Campaea perlata $(\mathrm{Gn})$. & 176 & 24.984 & 1,449 & 21.375 & $\mathrm{P}<0.001^{*}$ \\
\hline Tetracis cachexiata (Gn.) & 104 & 23.400 & 926 & 20.947 & $\mathrm{P}<0.001 *$ \\
\hline Itame pustularia $(\mathrm{Gn})$. & 1,716 & 13.254 & 3,035 & 12.287 & $\mathrm{P}<0.001^{*}$ \\
\hline Besma quercivoraria (Gn.) & 90 & 16.923 & 594 & 16.956 & $\mathrm{P}=0.961$ \\
\hline Besma endropiaria $(\mathrm{Gn})$. & 441 & 18.169 & 935 & 18.139 & $\mathrm{P}=0.581$ \\
\hline Malacosoma disstria (Hbn.) & 22 & 19.979 & 829 & 14.287 & $\mathrm{P}<0.001 *$ \\
\hline Heterocampa guttivitta (Wlk.) & 58 & 22.445 & 2,391 & 20.641 & $\mathrm{P}<0.001 *$ \\
\hline Nadata gibbosa (J.E. Smith) & 91 & 27.351 & 1,614 & 24.095 & $\mathrm{P}<0.001 *$ \\
\hline Hypoprepia fucosa (Hbn.) & 456 & 16.419 & 6,274 & 15.003 & $\mathrm{P}<0.001^{*}$ \\
\hline Halysidota tesselaris (J.E. Smith) & 163 & 22.907 & 2,311 & 21.994 & $\mathrm{P}<0.001 *$ \\
\hline Achatia distincta (Hbn.) & 41 & 16.290 & 10 & 16.220 & $\mathrm{P}=0.812$ \\
\hline Polia latex $(\mathrm{Gn})$. & 416 & 20.765 & 661 & 19.620 & $\mathrm{P}<0.001^{*}$ \\
\hline Morrisonia confusa $(\mathrm{Hbn})$. & 563 & 17.825 & 719 & 16.668 & $\mathrm{P}<0.001^{*}$ \\
\hline Allotria elonympha $(\mathrm{Hbn})$. & 93 & 19.491 & 738 & 19.239 & $\mathrm{P}=0.016$ \\
\hline Baileya opthalmica $(\mathrm{Gn})$. & 295 & 15.087 & 486 & 14.694 & $\mathrm{P}<0.001^{*}$ \\
\hline Acronicta ovata (Grt.) & 2,988 & 16.873 & 9,952 & 16.461 & $\mathrm{P}<0.001 *$ \\
\hline
\end{tabular}


Table 11. Average Larval Weights/plot (g).

\begin{tabular}{|c|c|c|c|c|c|}
\hline & All Larvae & Geometrids & Noctuids & Besma endropiaria & Polia latex \\
\hline \multicolumn{6}{|l|}{ Main Effects } \\
\hline Forest (GW / MON) & $0.1127 / 0.0555^{\star \star}$ & $0.0312 / 0.0240$ & $0.1430 / 0.0629^{\star \star}$ & $0.0278 / 0.0264$ & $0.1551 / 0.0506^{\star \star}$ \\
\hline Treatment (Ref / Bt / GC) & $0.0920 / 0.0780 / 0.0823$ & $0.0370 / 0.0223 / 0.0233$ & $0.0986 / 0.0970 / 0.1132$ & $0.0282 / 0.0235 / 0.0297$ & $0.1045 / 0.1111 / 0.0929$ \\
\hline \multicolumn{6}{|l|}{ Interaction } \\
\hline Forest X Treatment & NS & NS & NS & NS & NS \\
\hline & & & & & \\
\hline \multicolumn{6}{|l|}{ 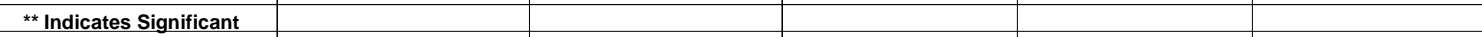 } \\
\hline \multicolumn{6}{|l|}{ Differences $(p<.05)$} \\
\hline & & & & & \\
\hline NS Indicates Not Significant & & & & & \\
\hline$(p>05)$ & & & & & \\
\hline
\end{tabular}

Table 12. Average Pupal Weights/plot (g).

\begin{tabular}{|c|c|c|c|}
\hline & All Pupae & Noctuids & Polia latex \\
\hline Main Effects & & & \\
\hline Forest (GW / MON) & $0.2657 / 0.2122^{\star \star}$ & $0.2855 / 0.2183^{\star \star}$ & $0.3036 / 0.2685^{\star \star}$ \\
\hline Treatment (Ref / Bt / GC) & $0.2527 / 0.2351 / 0.2291$ & $0.2468 / 0.2407 / 0.2680$ & $0.2736 / 0.2839 / 0.2992$ \\
\hline Interaction & & & \\
\hline Forest X Treatment & NS & NS & NS \\
\hline$\star \star$ Indicates Significant & & & \\
\hline Differences (p<.05) & & & \\
\hline NS Indicates Not Significant & & & \\
\hline$(\mathbf{p}>$.05) & & & \\
\hline
\end{tabular}

\section{Trends Indicated by Statistical Analyses:}

General trends in larval and adult count data, wing lengths, larval weights, and pupal weights are illustrated in subsequent graphs. Results of specific statistical analyses will be reviewed by initially focusing on generalities among the 19 species chosen for analysis in this study and on generalities within the geometrid and noctuid families. The following results are focused individually on each of the 19 selected species in this study. Depending on numbers of individuals collected, each ANOVA employed the maximum number of main effects (SEX, YEAR, FOREST, and TREATMENT), with PLOT assigned as random and nested within FOREST and TREATMENT. For interactions, Least Significant Difference (LSD) analysis was conducted and significant trends are stated in the subsequent text. All statements of significance in this regard are considered at the $\mathrm{P}<0.05$ level. 


\section{A. Selected Species Combined, Geometrids, and Noctuids}

\section{Larval Counts (1995-1998)}

The 19 selected species in this study comprised $49.3 \%$ of all of the larvae collected during 1995 1998 from foliage samples, with 5,921 individuals (the overall richness was 151 species). Canvas bands contributed an additional 1,271 individuals of the selected larval species. Geometrids and noctuids comprised $24.0 \%$ and $61.9 \%$, respectively for $85.9 \%$ of the individuals of the selected species collected under bands and from foliage. These figures compare with $21.7 \%$ geometrids and $41.0 \%$ noctuids, for $62.7 \%$ of all individual larvae captured from foliage.

Overall, the 3-way ANOVA indicates that larvae of the 19 species were significantly less abundant in the catches during the combined pretreatment years when compared to the combined treatment years $(\mathrm{P}=0.027)$. The YEAR $x$ FOREST interaction was also significant $(\mathrm{P}=0.002)$. The interaction for YEAR $\mathrm{x}$ FOREST indicates that significantly more larvae were captured on the MON during treatment years. When all larval species from foliage were analyzed (including species outside of the selected 19 in this study), the main YEAR effect shows a decrease in numbers during treatment years $(\mathrm{P}=0.039)$. Interestingly, the effect is not significant when gypsy moths are excluded (a drastic decline in gypsy moths was noted subsequent to 1995). The three graphs in Figure 3 aid in illustrating the general trends for larval counts during 1995-1998 for the selected 19 species. The first graph illustrates the average larval counts for all 19 species combined (7,192 individuals). The first 9 plots are located within the GW, while the remaining plots are located within the MON. The second graph in Figure 3 illustrates the average larval counts for the geometrids collected from each plot (1,726 individuals). A 3-way ANOVA indicates that only the YEAR x FOREST interaction was significant $(\mathrm{P}<0.001)$. Numbers were significantly higher during treatment years on the MON when compared to treatment years on the GW and to pretreatment years on the MON. The third graph in Figure 3 illustrates the average larval counts of noctuids collected from each plot. The trend appears similar to the first graph illustrating all selected species together. A 3-way ANOVA indicates a significant 
effect for YEAR $(\mathrm{P}=0.001)$, with an increase in individuals being attributed to treatment years. Interactions were not significant.

Similar analyses were conducted using data collected only during the first 5 weeks of the pretreatment and treatment sampling seasons. When the 19 selected species were combined (606 individuals), the effects for YEAR ( $\mathrm{P}=0.036)$, FOREST $(\mathrm{P}=0.15)$ and the YEAR x FOREST interaction $(\mathrm{P}=0.001)$ were significant. Pretreatment years showed significantly higher larval counts than treatment years. More individuals were collected from the GW when compared to the MON. The significant interaction was the result of lower numbers of GW larvae collected during treatment years. When non-target foliage larvae were analyzed from the same period (1,308 individuals), the resulting trends were similar. The effects of YEAR, FOREST, and the YEAR x FOREST interaction were significant $(\mathrm{P}=0.001, \mathrm{P}=0.012$, and $\mathrm{P}=0.002$; respectively). Though the YEAR $\mathrm{x}$ TREATMENT interactions were not significant at the $\mathrm{P}=0.05$ level, the two graphs in Figure 4 illustrate the trends.

Average weekly larval counts for 1997-1998 treatment years are illustrated in Figure 5. Comparisons among Ref plots, Bt plots, and GC plots are included. The general trend for all 19 species combined and for the noctuids illustrates a general increase in the numbers collected during the season. Trends appear similar for treatment groups. Geometrids, however, show an earlier period of prevalence which tapers off slightly during mid-season, with a slight increase toward the end of the sampling season. During treatment application weeks early in the season, geometrids on the $B t$ plots appeared somewhat less abundant when compared to GC and Ref plots. 

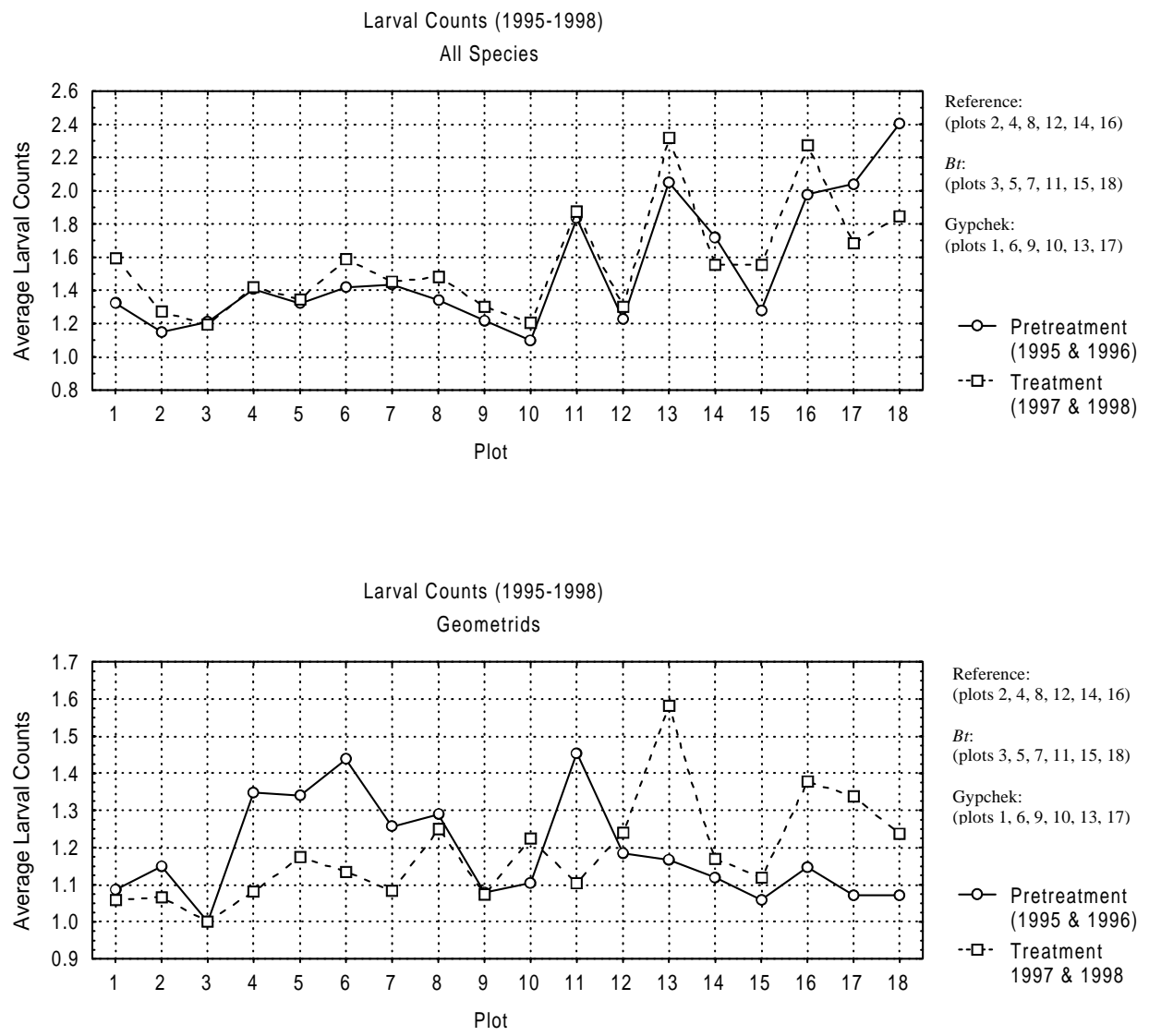

Reference:

(plots 2, 4, 8, 12, 14, 16)

$B t$ :

(plots 3, 5, 7, 11, 15, 18) Gypchek:

(plots 1, 6, 9, 10,13,17)

-o- Pretreatment (1995 \& 1996)

--ם- Treatment $1997 \& 1998$

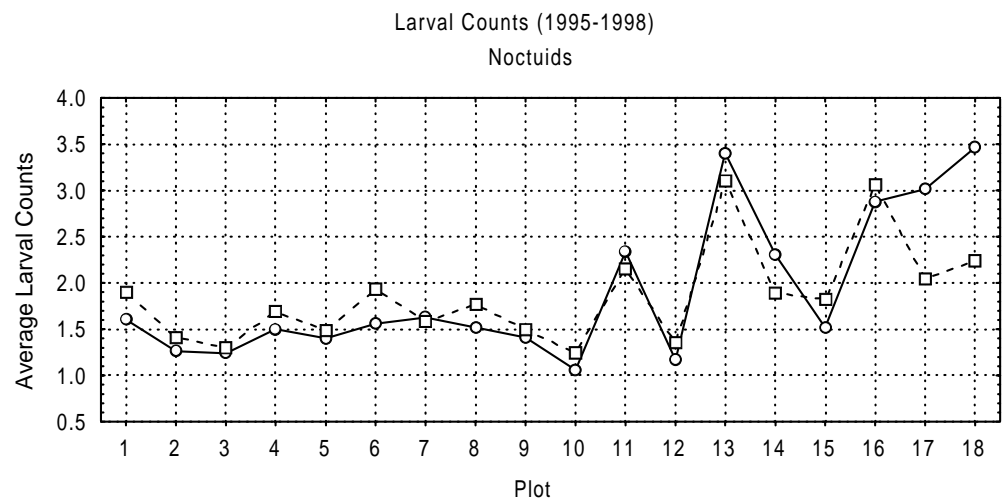

Reference:

(plots 2, 4, 8, 12, 14, 16)

$B t$

(plots 3, 5, 7, 11, 15, 18)

Gypchek:

(plots 1, 6, 9, 10, 13, 17)

-o- Pretreatment (1995 \& 1996)

- $\square$ - Treatment

(1997 \& 1998)

Figure 3. Average larval counts (1995-1998). Graphs indicate average larval counts for each plot during the 15-week sampling seasons for selected species combined, geometrids, and noctuids. George Washington National Forest plots are 1-9; Monongahela National Forest plots are 10-18. 

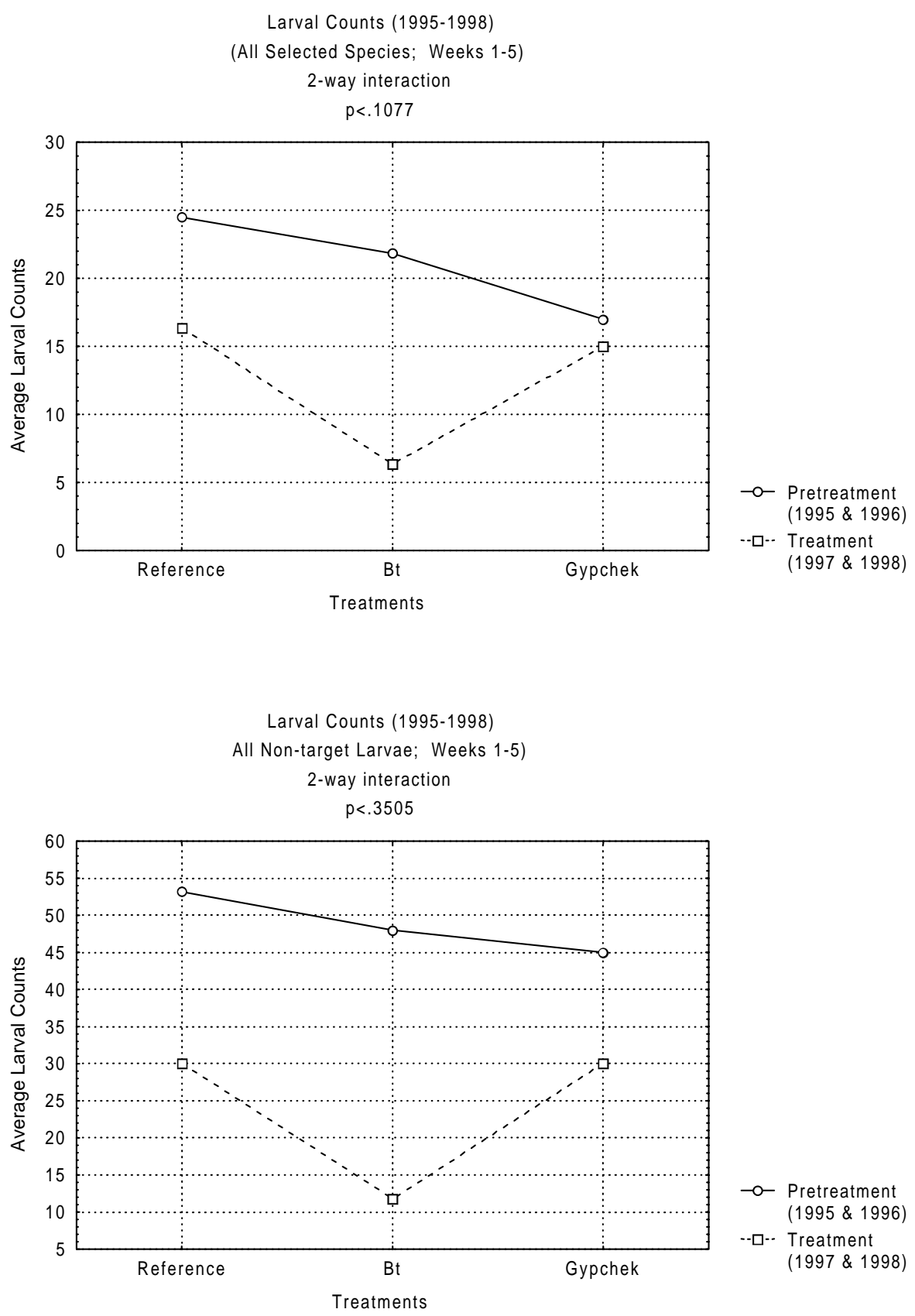

Figure 4. Average larval count interactions (1995-1998). Year $x$ Treatment interactions illustrated in the graphs are not significant at the $\mathbf{P}=\mathbf{0 . 0 5}$ level. The first graph illustrates average larval counts among treatment plots during pretreatment and treatment years for the 19 selected species combined during the first 5 weeks of the 15-week sampling season. The second graph illustrates similar trends for all nontarget lepidopteran larvae collected from foliage from the same plots during the same period. 

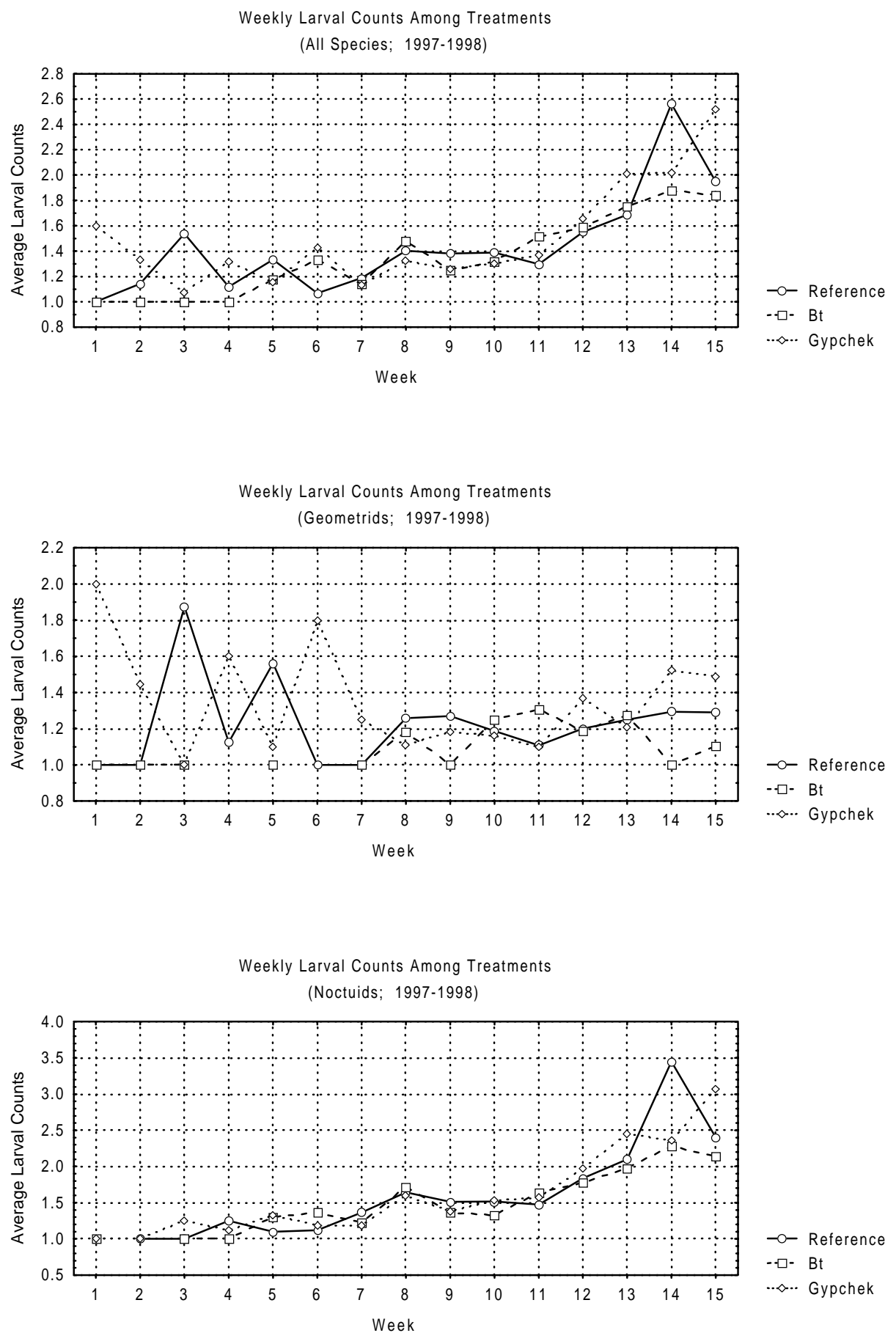

Figure 5. Average weekly larval counts (1997-1998). Graphs indicate average weekly larval counts for each treatment category for the selected species combined, geometrids, and noctuids, collected during treatment years. 


\section{Adult Counts (1995-1998)}

Total species richness of adult macrolepidopterans collected from light traps during the four years was 509. The 19 selected species in this study comprised $26.4 \%(104,079$ individuals) of the total adult count, which was 394,720 individuals. Within the 19 selected species, geometrids comprised $28.8 \%$ and noctuids comprised $43.4 \%$ for a total of $72.2 \%$ of the selected individuals. Overall, these figures compare with $23.9 \%$ geometrids and $58.2 \%$ noctuids, for a total of $82.1 \%$ of the total light trap catch (all species captured).

The overall 3-way ANOVA for the 19 selected species analyzed as a whole indicates significant main effects for both YEAR and FOREST ( $\mathrm{P}=0.002$ and $\mathrm{P}=0.023$, respectively). As with larval counts, the YEAR effect showed a higher number of adults collected during treatment years. The FOREST effect is such that numbers were higher on the MON. A significant FOREST x YEAR interaction effect $(\mathrm{P}=0.006)$ was also seen. The MON counts during treatment years were significantly higher than the others. Figure 6 illustrates the trends for the adult counts as a whole in the first graph. The second and third graphs correspond to the geometrids and noctuids, respectively. Analysis of the geometrids (29,967 individuals) revealed a significant main effect for FOREST $(\mathrm{P}=0.003)$ and an interaction for YEAR $\mathrm{x}$ FOREST $(\mathrm{P}=0.012)$. The main effect shows higher numbers on the MON. The interaction indicates that the treatment MON adults are again significantly higher in number than the others. The noctuids (45,189 individuals) showed only a significant main effect for YEAR $(\mathrm{P}=0.004)$, with the higher numbers again seen during treatment years. 

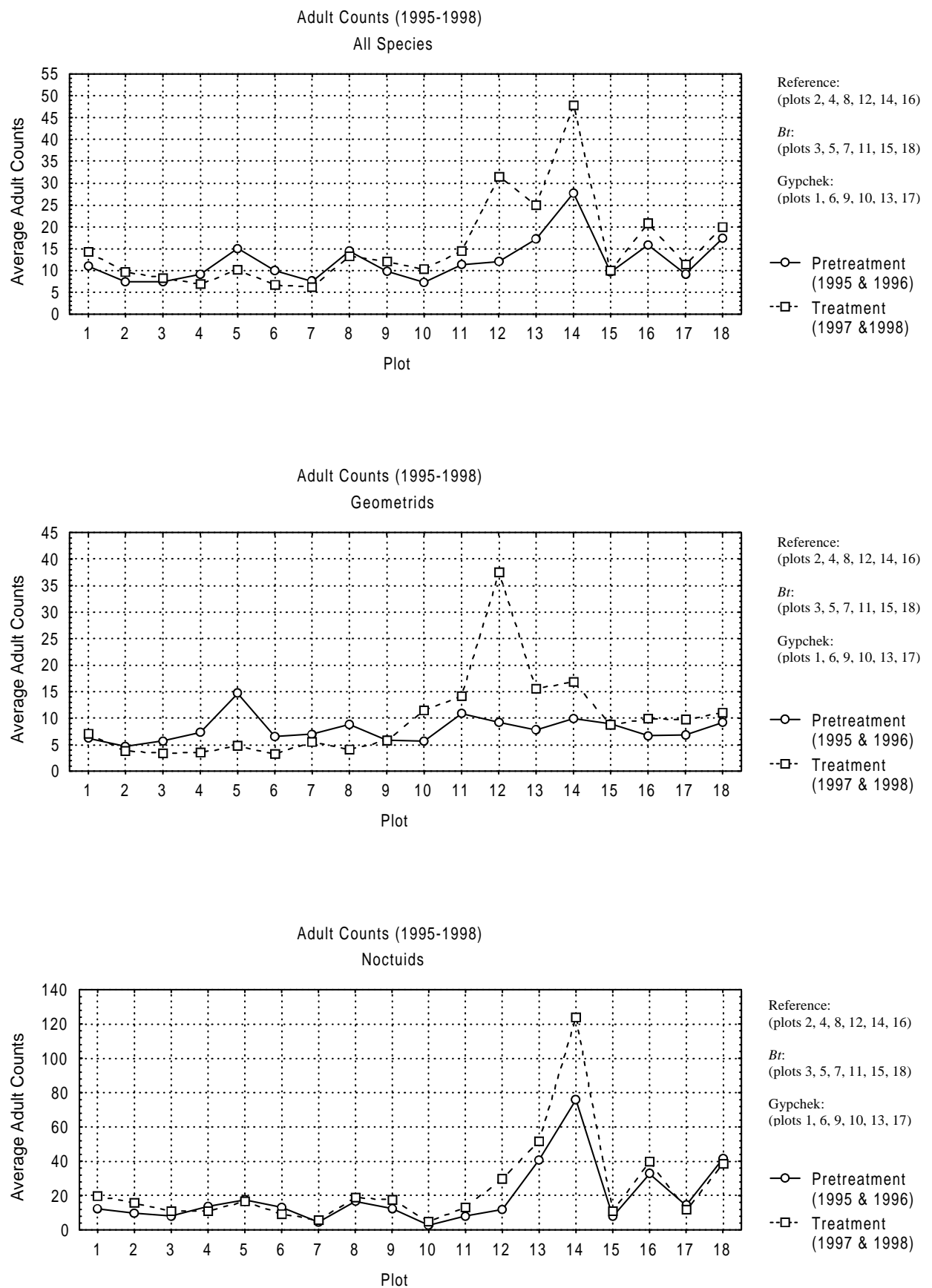

Reference:

(plots 2, 4, 8, 12, 14, 16)

$B t:$

(plots 3, 5, 7, 11, 15, 18)

Gypchek:

(plots 1, 6, 9, 10,13,17)

-o- Pretreatment (1995 \& 1996)

--- Treatment

(1997 \& 1998)

Figure 6. Average adult counts (1995-1998). Graphs indicate average adult counts for each plot during the 15-week sampling seasons for selected species combined, geometrids, and noctuids. George Washington National Forest plots are 1-9; Monongahela National Forest plots are 10-18. 


\section{Adult Counts (1995 \& 1998)}

For the first pretreatment year (1995) and the second treatment year (1998), all adults of the 19 selected species collected from light traps were separated from the remaining specimens in the catches and sexed. A 4-way ANOVA was utilized to assess these data. The model was designed as previously described for analyzing count data; however SEX was added as a main effect. For the 19 species combined as a whole, 9,831 females and 38,277 males were identified. Significant main effects were noted for SEX and for FOREST $(\mathrm{P}<0.001$ and $\mathrm{P}=0.015$, respectively). The SEX effects signify that many more adult males were collected than adult females. The FOREST effect shows that more adults were collected on the MON than on the GW. A significant interaction for YEAR x FOREST $(\mathrm{P}=0.033)$ was demonstrated indicating that the MON moths were significantly higher in number during 1998 than the GW during both years and the MON during 1995. Figure 7 illustrates the general trends for female and male counts during 1995 and 1998. The first graph illustrates the trends for the combined 19 species. The second and third graphs illustrate the trends for the geometrids and noctuids, respectively.

A total of 4,645 female and 12,595 male geometrids was counted and sexed (17,240 total). Analysis indicates similar results to those of the 19 species combined. The main effects of SEX and FOREST were significant $(\mathrm{P}<0.001$ and $\mathrm{P}=0.016$, respectively). The YEAR $x$ FOREST interaction was likewise significant $(\mathrm{P}=0.028)$, indicating the MON moths were significantly greater in number during 1998 than the $\mathrm{GW}$ in 1995 and 1998, and the MON in 1995. A total of 4,396 female and 12,263 male noctuids was counted and

sexed. Analysis indicated that the main effects of SEX and YEAR were significant $(\mathrm{P}<0.001$ and $\mathrm{P}=0.012$, respectively). Many more males were captured. More adults were captured during 1998 than in 1995.

Figure 8 illustrates the average weekly counts among treatment plots for adults collected during 1998. Trends appear comparable among treatments with a general trend for an increase later in the season for the noctuids. 
Female and Male Adult Counts (1995 \& 1998)

All Species
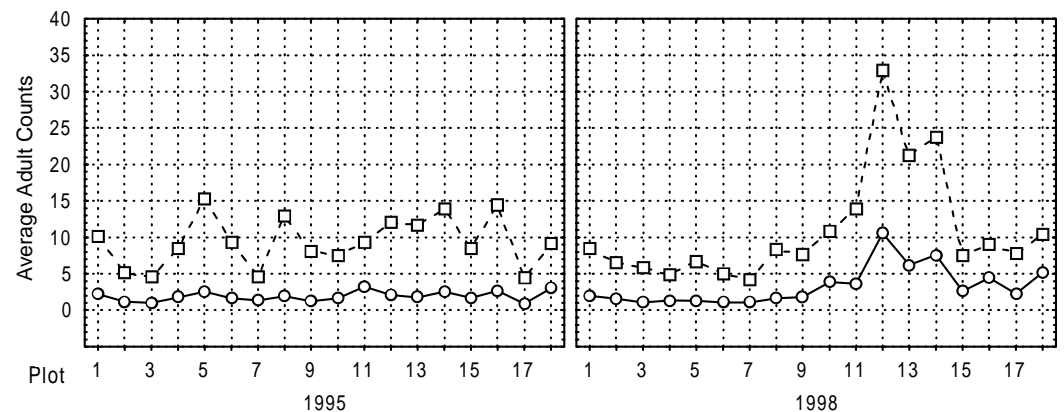

Reference:

(plots 2, 4, 8, 12, 14, 16)

(plots $3,5,7,11,15,18$ )

Gypchek:

(plots 1, 6, 9, 10,13,17)

Female and Male Adult Counts (1995 \& 1998)

Geometrids
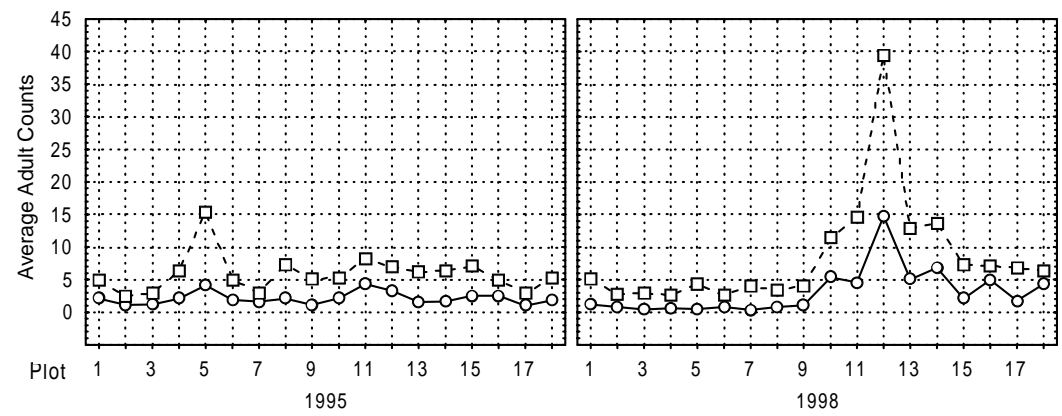

Reference:

(plots 2, 4, 8, 12, 14, 16)

(plots 3, 5, 7, 11, 15, 18)

Gypchek:

plots $1,6,9,10,13,17)$

- Females

-- - Males

Female and Male Adult Counts (1995 \& 1998)

Noctuids
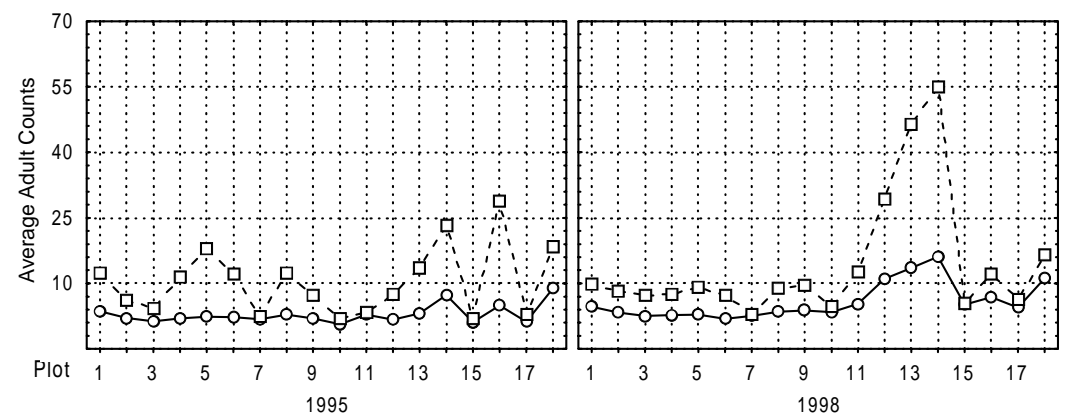

Reference:

(plots $2,4,8,12,14,16)$

(plots $3,5,7,11,15,18$

Gypchek:

(plots $1,6,9,10,13,17$ )

$\rightarrow$ Females

- - - Males

Figure 7. Average female and male adult counts (1995 \& 1998). Graphs illustrate average plot counts for females and males collected during the 15-week sampling season during the 1995 pretreatment and 1998 treatment years. George Washington National Forest plots are 1-9; Monongahela National Forest plots are 10-18. 

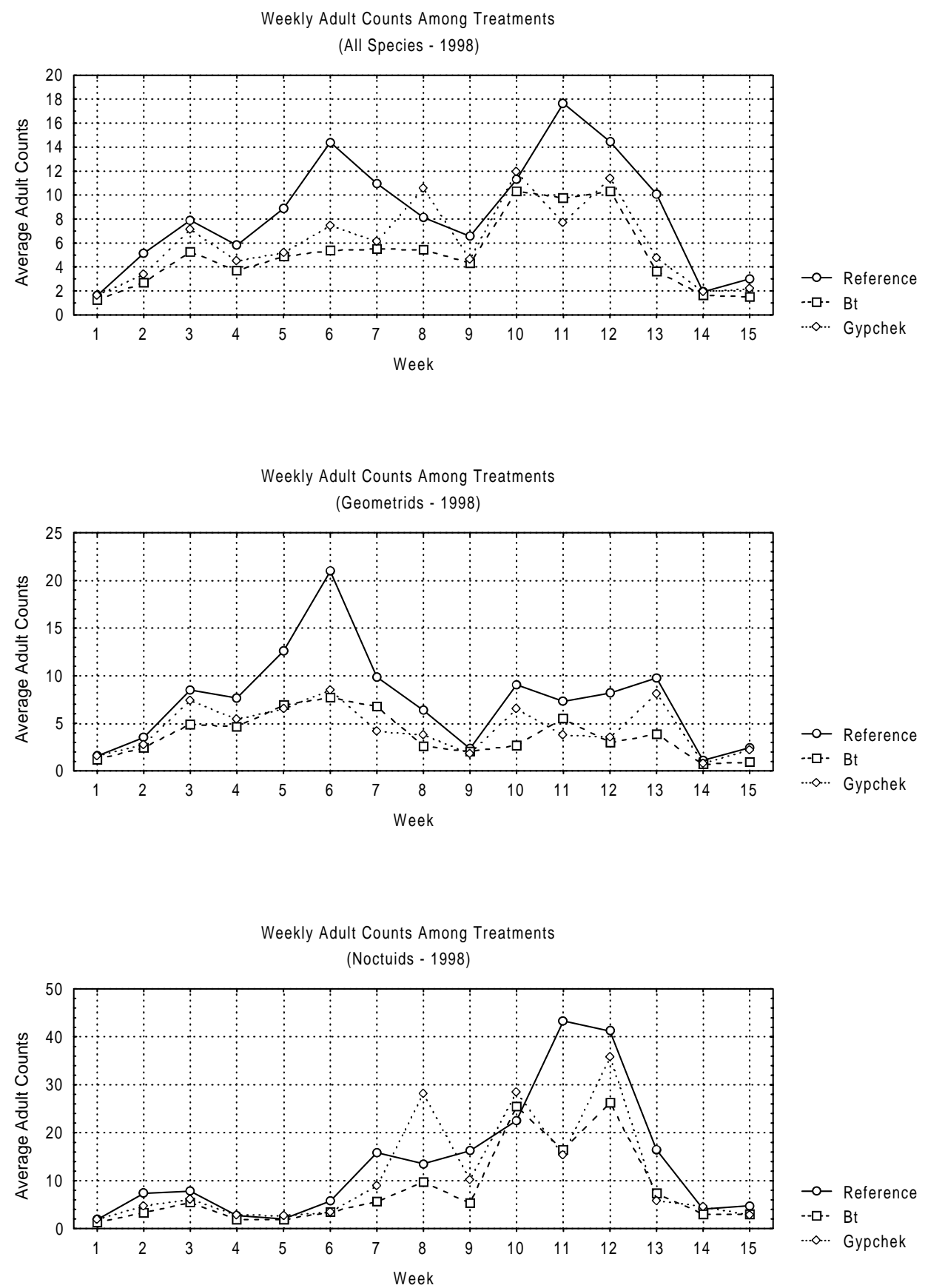

Figure 8. Average weekly adult counts (1998). Graphs illustrate average weekly adult counts in catches from each treatment category during the 1998 treatment year. 


\section{Adult Wing Lengths (1995 \& 1998)}

For the first pretreatment year (1995) and the second treatment year (1998), most intact adults of the 19 selected species were measured for wing length in an effort to determine potential sublethal treatment effects. A total of 40,674 individuals was measured. As with the count data, a 4-way ANOVA was used to assess the data. In this case, however, wing lengths were used as the dependent variable. The variables SEX, YEAR, FOREST, and TREATMENT were the main effects. As previously described, PLOT was nested within FOREST and TREATMENT and assigned as random. For the combined 19 species, the main effect for YEAR was significant $(\mathrm{P}<0.001)$. The adult moths were larger during 1998 than in 1995. Two of the interactions were also significant, SEX x YEAR and SEX x FOREST $(\mathrm{P}=0.003$ and $\mathrm{P}=0.001$, respectively). As previously discussed, LSD analyses were again conducted on significant interactions indicated by the 4-way ANOVA. All statements of significance in this regard are at the $\mathrm{P}<0.05$ level. In this case, wing lengths of the moths of both sexes collected during 1995 were significantly shorter than the wing lengths of the moths collected during 1998. Though male wing lengths were significantly longer during 1995 than female wing lengths, the differences were not significant during 1998. Wing lengths of females on the GW were significantly longer overall than wing lengths of females on the MON, while males on the GW showed significantly shorter wings than males on the MON. Finally, male wing lengths on the MON were significantly longer than female wing lengths on the MON. [It should be noted that males did not possess significantly longer wings than females within a species (except for Melanolophia canadaria); typically females possessed significantly longer wings than males.] General trends for the combined 19 species can be seen in the first graph illustrated by Figure 9.

For geometrids, 15,089 individuals were measured. The YEAR main effect showed a significant increase in overall wing length during 1998 ( $\mathrm{P}<0.001)$. Several interactions were also significant. The interaction for YEAR x SEX ( $\mathrm{P}=0.001)$ showed longer wing lengths in both sexes during treatment years, but female wing lengths increased significantly more than males. The SEX x FOREST interaction 
$(\mathrm{P}<0.001)$ showed female wing lengths were shorter on the MON than on the GW, while male wing lengths were longer on the MON than on the GW. For the combined geometrids, female wing lengths on the GW were significantly longer than male wing lengths. On the MON, male wing lengths were significantly longer than female wing lengths. A FOREST x YEAR interaction $(\mathrm{P}=0.037)$ showed that the wing lengths of 1998 moths were significantly longer than 1995 moths in both forests. In 1995, the Mon moths possessed wings significantly longer than the GW moths. A significant SEX x YEAR x FOREST interaction was also seen $(\mathrm{P}=0.010)$. General trends indicated that both males and females increased in wing length size in both forests from 1995 to 1998 . Females increased most on the GW, while males increased most on the MON. The second graph in Figure 9 illustrates the trends for the geometrids.

For noctuids, 13,688 individuals were measured. Significant main effects occurred for SEX and for FOREST $(\mathrm{P}<0.001$ and $\mathrm{P}=0.007$, respectively). Females possessed wings significantly longer than males. Moths with smaller wings were collected from the MON when compared to moths collected from the GW. The SEX x FOREST interaction $(\mathrm{P}=0.008)$ indicated that although wings of both sexes were smaller on the MON, males exhibited more of a size difference than females. The FOREST x YEAR interaction $(\mathrm{P}=0.001)$ showed that the GW and MON moths were of similar wing size during 1995, but wing lengths of moths on the MON were smaller during 1998. The SEX x FOREST x YEAR interaction $(\mathrm{P}=0.012)$ showed that both males and females had an increase in wing length on the GW from 1995 to 1998, but males showed less of an increase than females. The MON forest showed the reverse trend, with both sexes possessing shorter wings during 1998 when compared to 1995 catches. Female wing lengths were longer than male wing lengths in both forests during both years. The third graph in Figure 9 illustrates the general trends.

Figure 10 illustrates the general trends during 1998 for weekly wing lengths among all species combined, geometrids, and noctuids. Ref plots are compared with $B t$ and GC plots. In each case, the trend appears to indicate that longer moths are more prevalent earlier in the season than later in the season. No differences were found among treatments. 

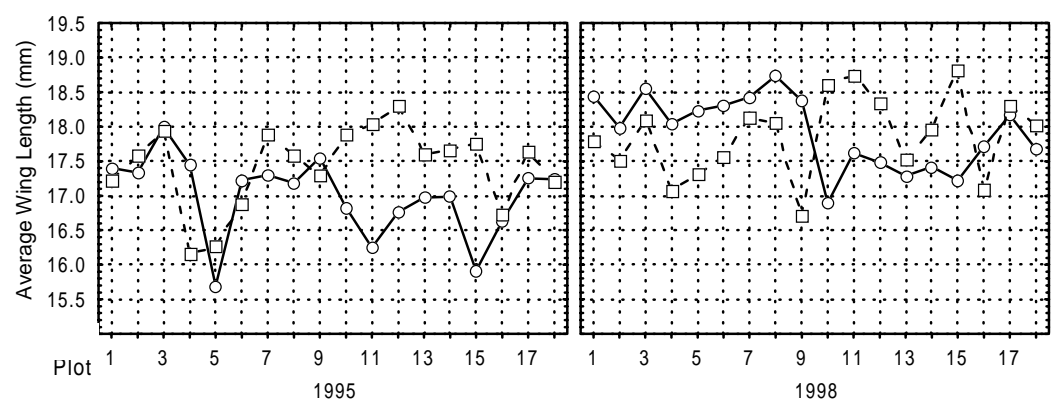

Reference:

(plots 2, 4, 8, 12, 14, 16)

$B t$ :

(plots 3, 5, 7, 11, 15, 18)

Gypchek:

(plots $1,6,9,10,13,17)$

Female and Male Wing Lengths (1995 \& 1998)

Geometrids
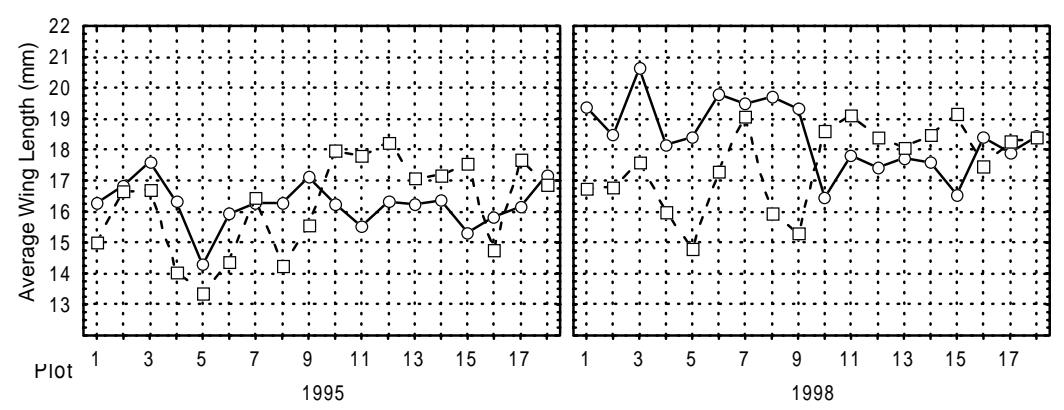

Reference:

(plots $2,4,8,12,14,16)$

$B t:$

(plots 3, 5, 7, 11, 15, 18)

Gypchek:

(plots 1, 6, 9, 10, 13, 17)

$-\infty$ Females

$-\square$ - Males

Female and Male Wing Lengths (1995 \& 1998) Noctuids
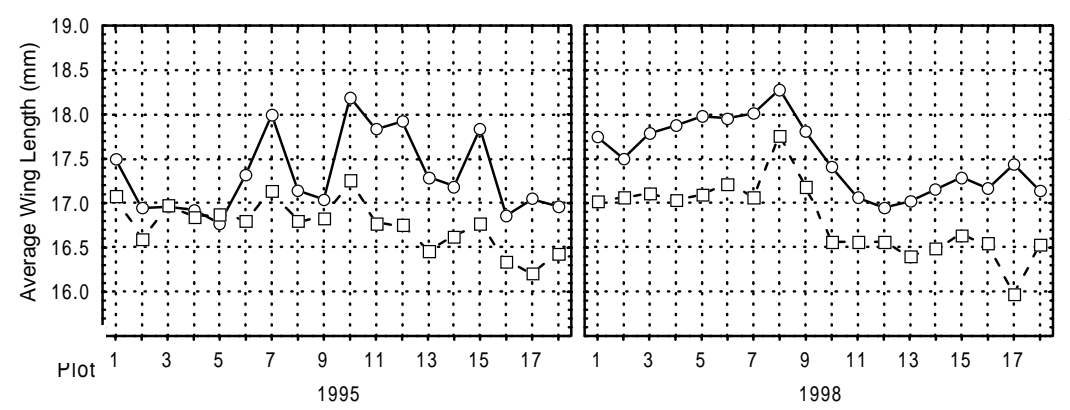

Reference: (plots 2, 4, 8, 12, 14, 16) $B t:$

(plots $3,5,7,11,15,18)$

Gypchek:

(plots 1, 6, 9, 10, 13, 17)

$\rightarrow$ Females

$-\square$ - Males

Figure 9. General average wing lengths (1995 and 1998). Graphs indicate average wing lengths of males and females collected from each plot during 1995 pretreatment and 1998 treatment years. George Washington National Forest plots are 1-9; Monongahela National Forest plots are 10-18. 

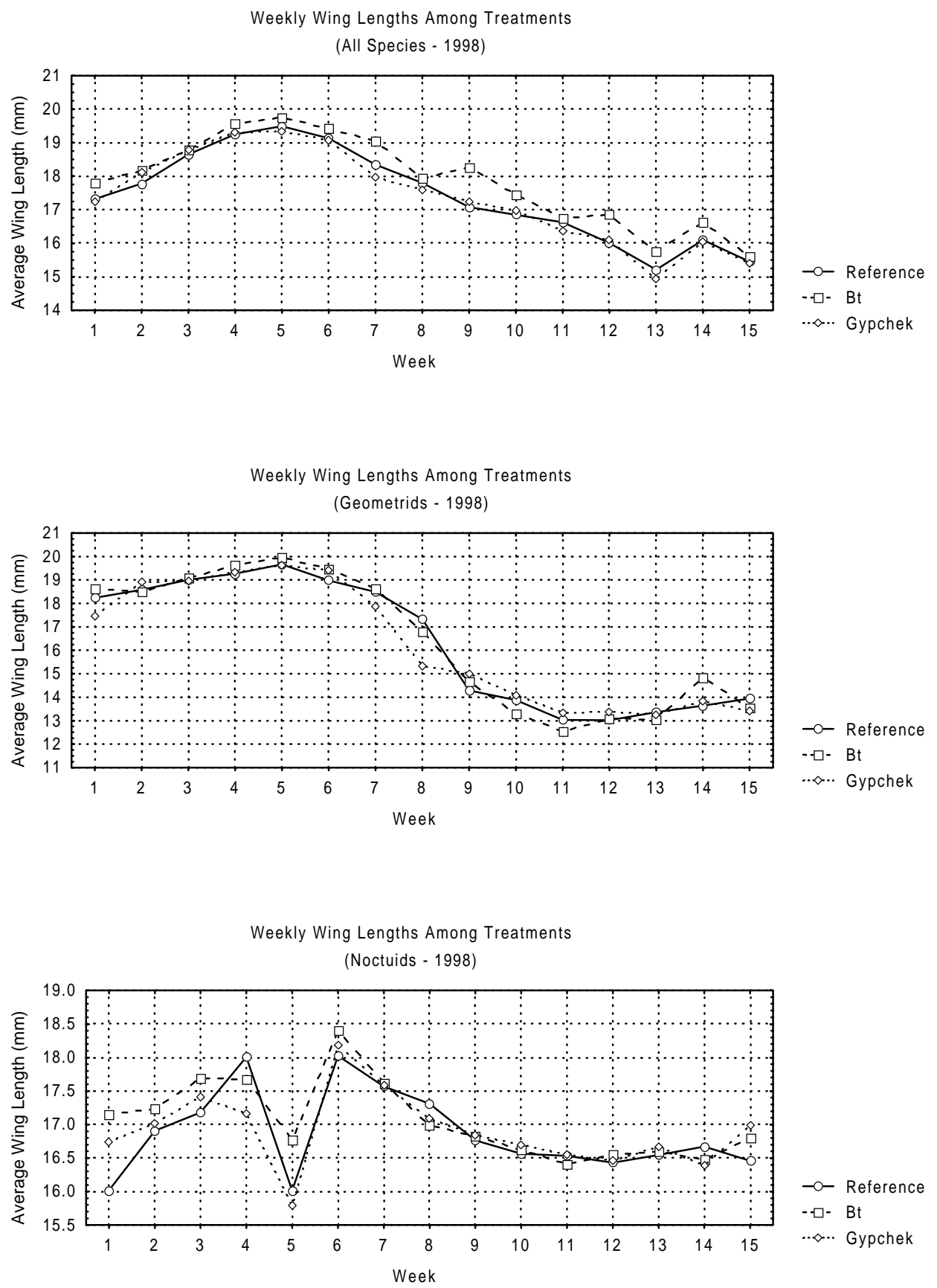

Figure 10. Average weekly wing lengths (1998). Graphs illustrate average weekly wing lengths of moths collected from each treatment category during the 1998 treatment year for the selected species combined, the geometrids, and the noctuids. 


\section{Larval and Pupal Sizes (1997-1998)}

Larvae of the 19 selected species collected after the seventh week of the 1997 sampling season and during the normal 15 weeks of the 1998 sampling season were weighed with a Mettler balance to the nearest $10,000^{\text {th }}$ of a gram. First instar larvae were not weighed. A 2-way ANOVA was conducted testing the main effects of FOREST and TREATMENT. As in previous analyses, PLOT was nested within FOREST and TREATMENT and assigned as random. A total of 1,223 larvae was included in the analysis. Of this total, 406 were geometrids and 612 were noctuids ( $83.2 \%$ of the total number weighed). The average geometrid weight was $0.0278 \mathrm{~g}$, while the average noctuid weight was $0.0975 \mathrm{~g}$.

Weekly larval weights of all the 19 species combined among treatment plots are illustrated in Figure 11 as the first graph. Early and late season larvae appeared to be heavier than mid-season larvae across all treatment types. Likewise, weekly larval weights showed a larger size for noctuid larvae collected during the early and later portions of the sampling seasons (see the second graph in Figure 11). For the ANOVA, geometrids showed no significant effects for FOREST or TREATMENT. The total 19 species combined, and the noctuids, however, showed a significant FOREST effect, with the larger larvae collected from the GW and the smaller from the $\operatorname{MON}(\mathrm{P}=0.007$ and $\mathrm{P}=0.003$, respectively). The first graph in Figure 12 illustrates this trend.

Pupae of those larvae of the 19 species reared in captivity were weighed within a few days of tanning. Of 528 weighed, 111 were geometrids and 336 were noctuids (84.6\% of the total number of pupae). Reviewing the 19 species combined and the noctuids, both showed significantly larger pupae from individuals collected from the $\mathrm{GW}$ than from the $\mathrm{MON}(\mathrm{P}=0.002$ and $\mathrm{P}<0.001$, respectively). Geometrids showed no significant differences in pupal weights between forests. The second graph in Figure 12 illustrates these trends. 

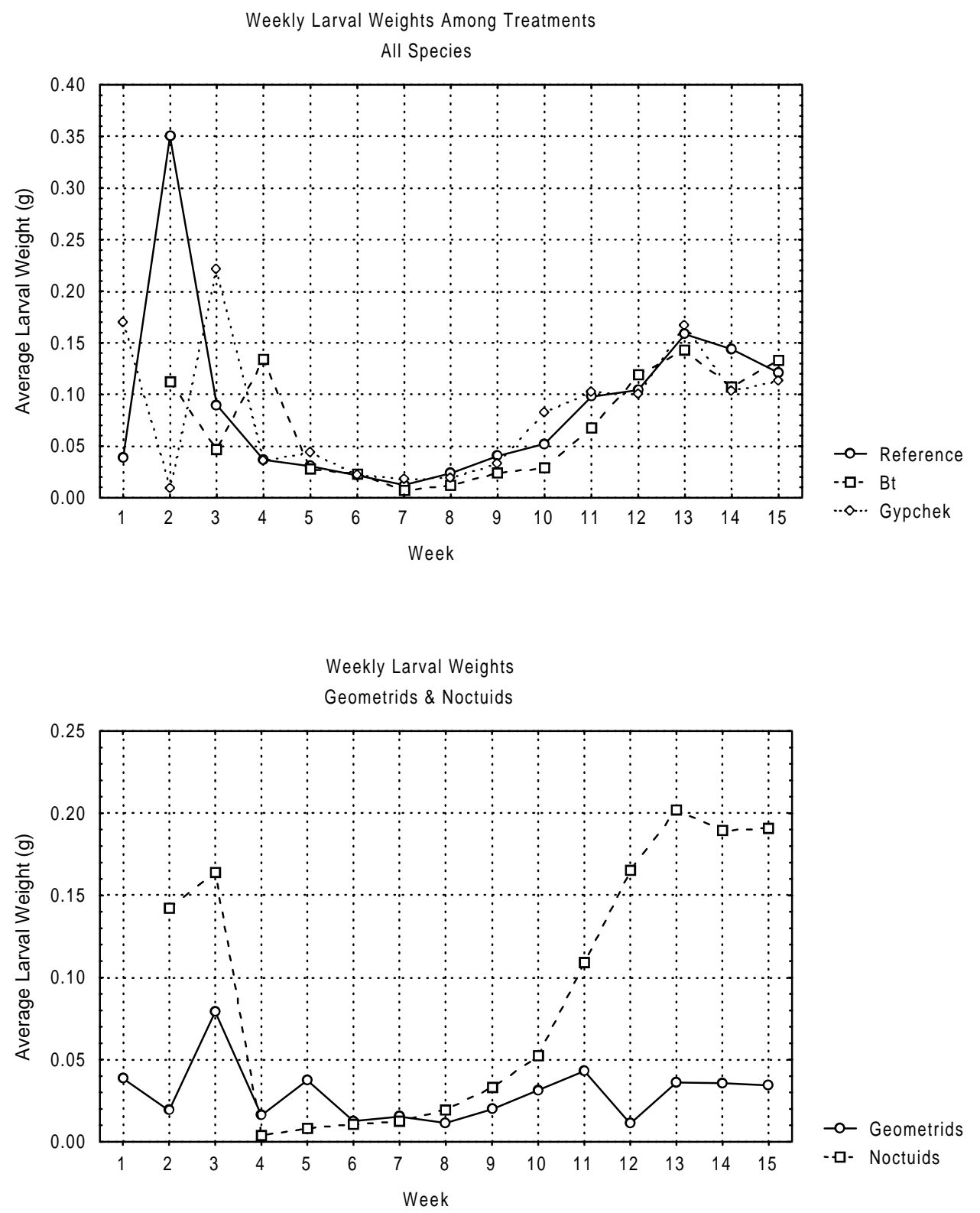

Figure 11. Weekly larval weights (1997-1998). The first graph illustrates weekly larval weights for selected species combined among treatment categories. The second graph illustrates average weekly larval weights of combined geometrids and combined noctuids. 

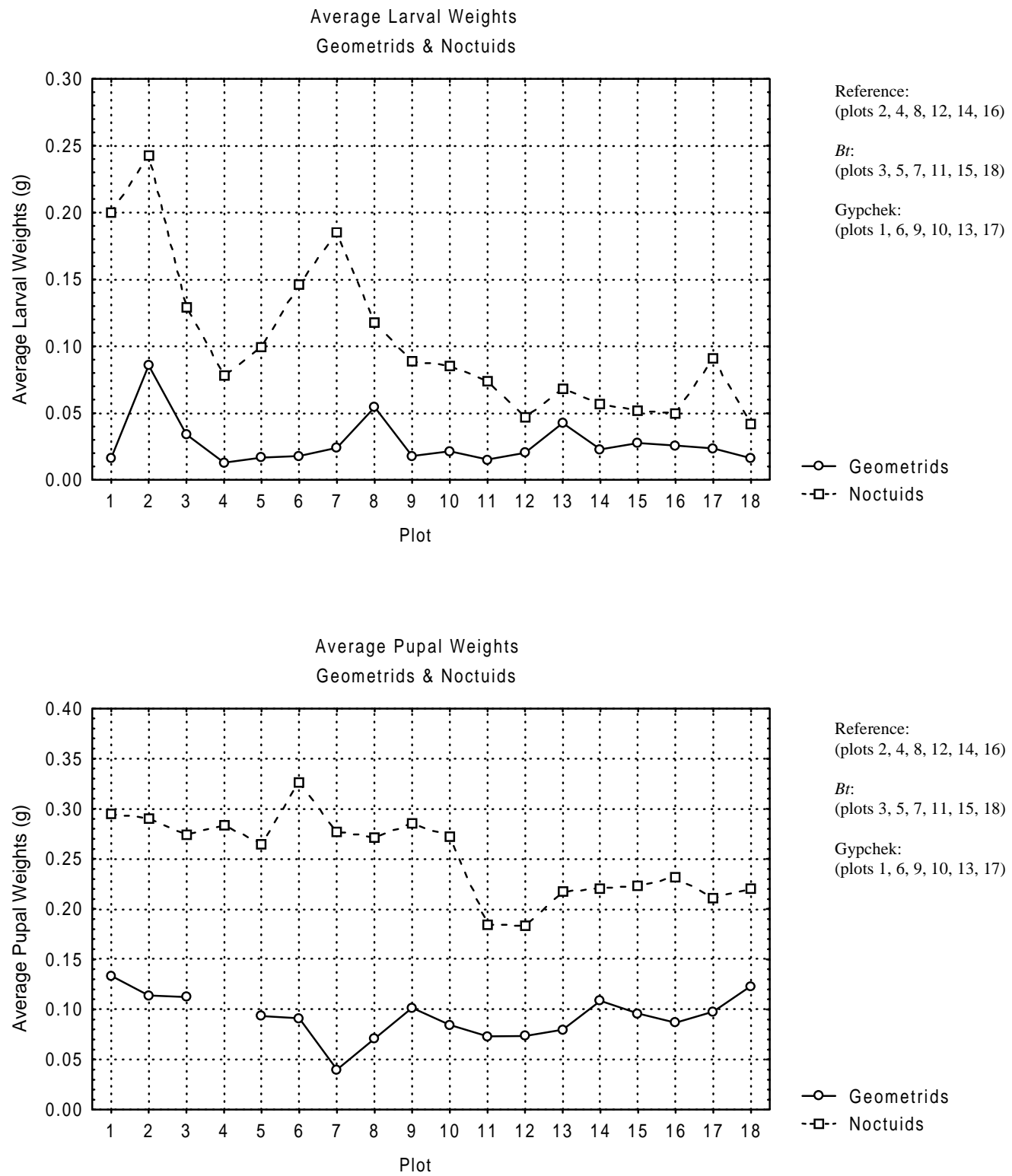

Figure 12. Average larval and pupal weights (1997-1998). The first graph illustrates average larval weights for each plot from 1997 and 1998 catches. The second graph illustrates average pupal weights from these larvae. George Washington National Forest Plots are 1-9; Monongahela National Forest Plots are 10-18. 


\section{B. Statistical Results for Individual Species:}

\section{Melanolophia canadaria}

A total of 316 larvae was collected during 1995-1998. Most of the larvae were collected during the first five weeks in the GW, while the bulk of the larvae collected from the MON was during weeks 8-11 (see the first graph in Figure 13). The 3-way ANOVA showed a significant decrease in larval numbers from pretreatment to treatment years $(\mathrm{P}=0.008)$. A significant interaction $(\mathrm{P}=0.030)$ was seen for $\mathrm{YEAR} \mathrm{x}$ FOREST with significantly less individuals collected from the MON than from the GW during the pretreatment years. The second graph in Figure 13 further illustrates these trends.

A total of 1,892 adult moths was collected during 1995-1998. Though the numbers of moths collected each week from the GW remained fairly constant, there were more drastic fluctuations in catch size in the MON. The bulk of moths collected during treatment years appears to have occurred earlier than during pretreatment years in the MON (see the first graph in Figure 14). The 3-way ANOVA indicated a significant main effect for FOREST ( $\mathrm{P}=0.006)$, with more moths collected from the MON than from the GW. A FOREST x YEAR interaction was also significant $(\mathrm{P}=0.032)$, indicating the increase in numbers was attributed to significantly more moths collected from the MON during the treatment years. The second graph in Figure 14 further illustrates these trends.

During the 1995 pretreatment year and the 1998 treatment year, 194 female and 888 male adult moths were identified. A 4-way ANOVA on this count data indicated that the SEX difference was significant as was the main effect for FOREST $(\mathrm{P}<0.001$ and $\mathrm{P}=0.010$, respectively). Significantly more adults were collected from the MON. A significant SEX x YEAR interaction $(\mathrm{P}=0.028)$ indicated a significant increase in the number of males collected from 1995 to 1998, though females remained relatively constant in number. A SEX x FOREST interaction $(\mathrm{P}=0.028)$ indicated the males were significantly more abundant in catches in the MON. A FOREST x YEAR interaction $(\mathrm{P}=0.004)$ indicated the increase in number was during 1998. A significant SEX x YEAR x FOREST interaction $(\mathrm{P}=0.019)$ further corroborates 
these trends with the increased male number in the MON being significant during 1998 when compared to all other statistical comparisons. The trends are illustrated in the first graph in Figure 15.

Due to the low number of intact females in the various groups (especially in the MON), a 3-way ANOVA was conducted on the males alone for wing length comparisons. Analysis indicated a significant increase in wing length in males from 1995 to 1998 ( $\mathrm{P}=0.008)$. The second graph in Figure 15 illustrates the trend for male wing lengths as well as the trend for females. Interestingly, a student's t-test conducted on wing lengths indicated that males were significantly longer than females $(18.449$ and $17.633 \mathrm{~mm}$, respectively; $\mathrm{P}<0.001)$. An ANOVA using only SEX and FOREST indicated that the MON females had significantly smaller wings than the GW females. Otherwise, males and females were of similar size. 

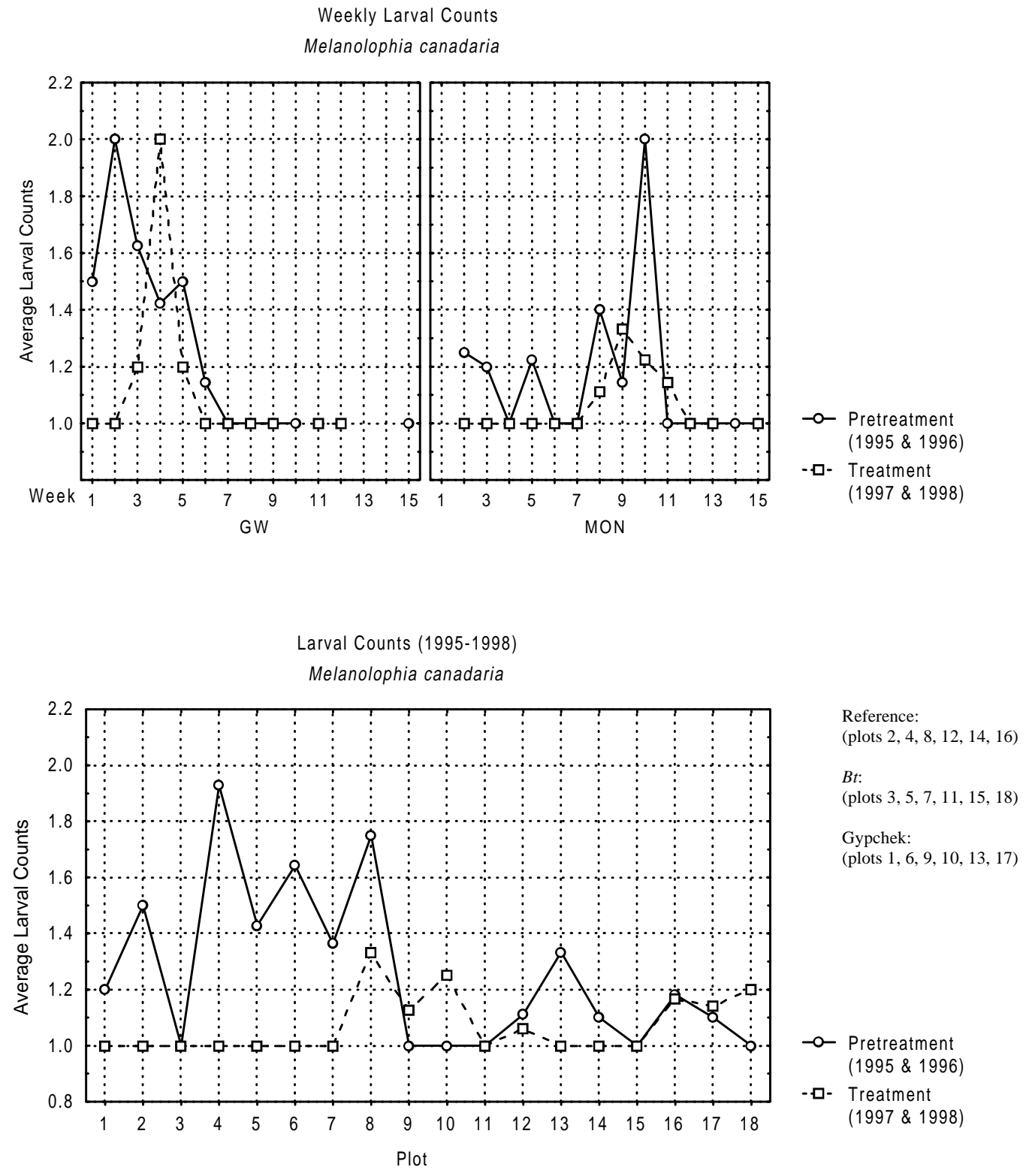

Figure 13. Larval counts (1995-1998). Graphs illustrate average weekly and average plot counts for Melanolophia canadaria larvae collected during pretreatment and treatment years. George Washington National Forest plots are 1-9; Monongahela National Forest Plots are 10-18. 

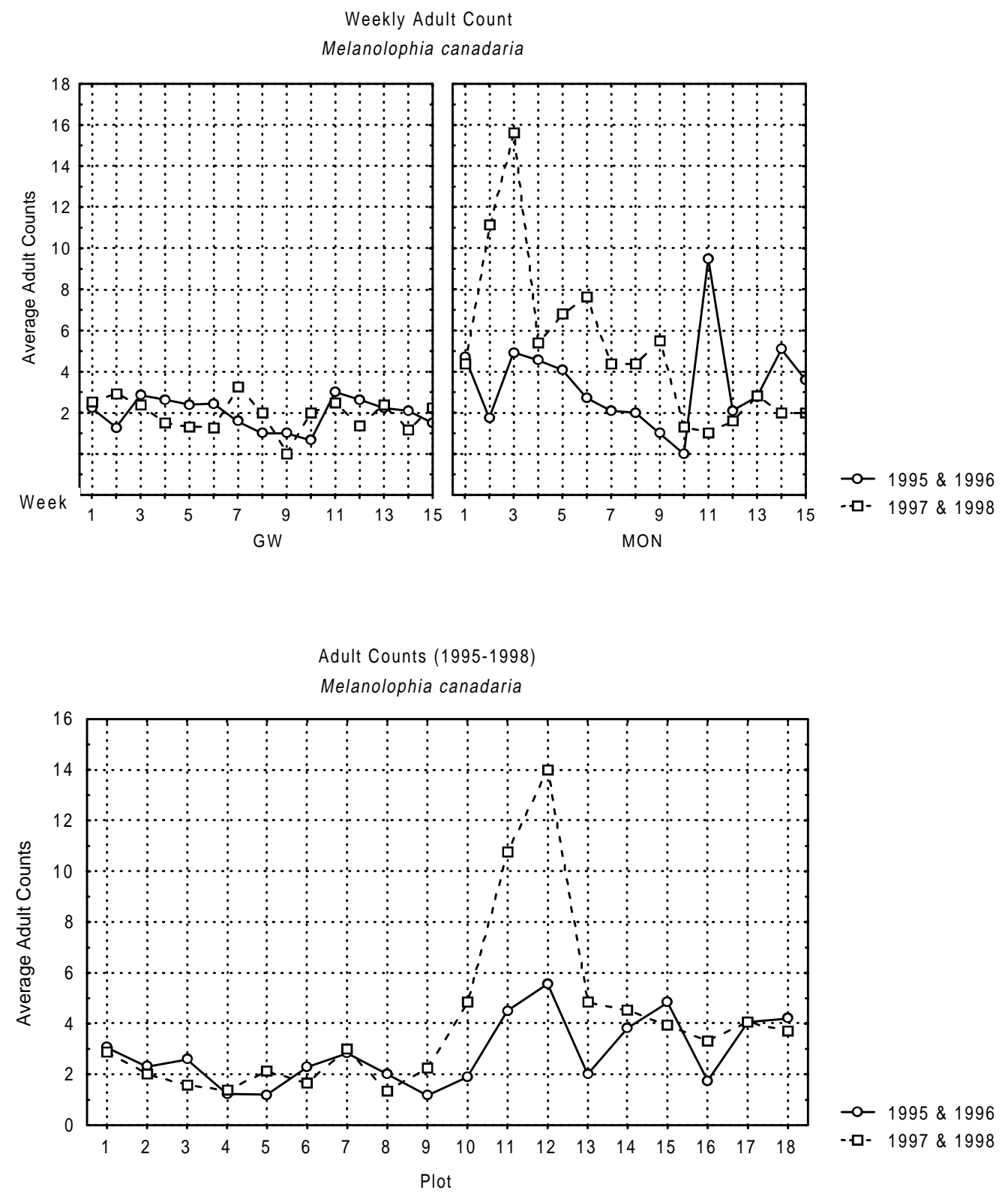

Figure 14. Adult counts (1995-1998). Graphs illustrate average weekly and average plot counts for Melanolophia candadaria adults collected during pretreatment and treatment years from the George Washington National Forest (plots 1-9) and Monongahela Natinal Forests (plots 10-18). 

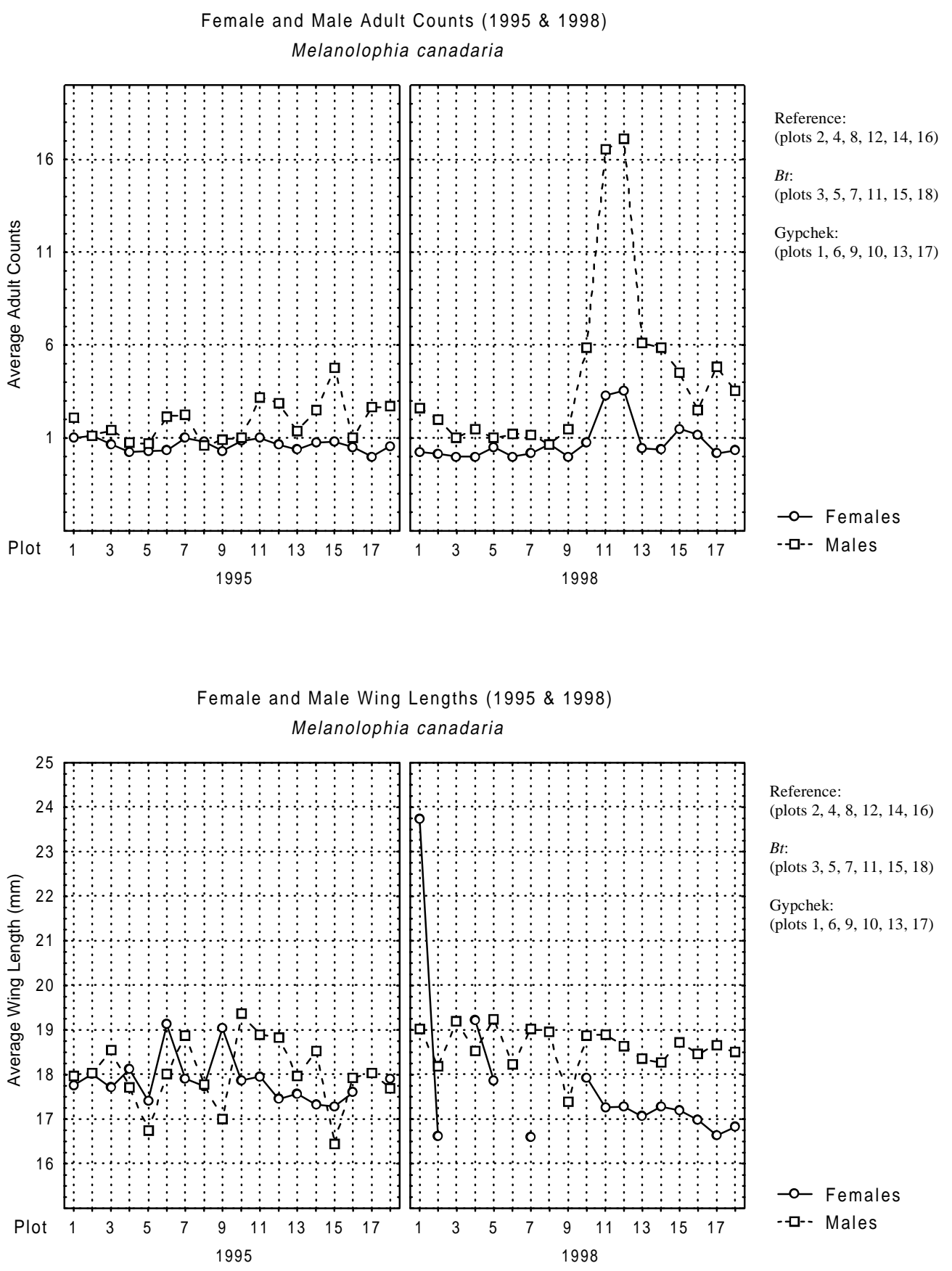

Figure 15. Female and male adult counts and wing lengths (1995 \& 1998). Graphs illustrate average plot counts for male and female Melanolophia canadaria adults captured during the 1995 pretreatment and 1998 treatment years from the George Washington National Forest (plots 1-9) and the Monongahela National Forest (plots 10-18). 


\section{Hypagyrtis unipunctata}

A total of 220 larvae and 2,835 adults was collected during 1995-1998. The bulk of larvae were collected early in the season and again near weeks 10 and 11 from both forests during 1995 \& 1996 (pretreatment). During 1997 \& 1998 (treatment), larvae appeared more prevalent during weeks 8 and 9. The early season peak was not apparent during these years. The first graph in Figure 16 illustrates the trends. Adults appeared to be most prevalent in catches between weeks 3 to 11 on the GW during pretreatment and treatment years, while the MON moths were more prevalent during weeks 7 to 13 (see second graph of Figure 16).

The 3-way ANOVA conducted on larval catches indicated no significant main effects; however a YEAR x FOREST interaction indicated that significantly more larvae were captured in the MON during the treatment years than during pretreatment years. The first graph in Figure 17 illustrates this trend.

The ANOVA conducted on adult moths showed a significant YEAR effect $(\mathrm{P}=0.005)$ such that fewer adults were collected during treatment years (see second graph in Figure 16). A YEAR x TREATMENT effect was also indicated $(\mathrm{P}=0.017)$. During treatment years, $B t$ plots yielded less moths than both Ref and GC plots. The difference between $B t$ plots during treatment years was also significant when compared to GC plots from treatment years. However, the Ref plots were not significantly different from other plots during pretreatment or treatment years. The trends are illustrated in the third graph of Figure 17.

During 1995 and 1998, 76 females and 1,055 males were identified. Significant SEX and YEAR main effects were indicated by a 4-way ANOVA $(\mathrm{P}<0.001$ and $\mathrm{P}=0.047$, respectively). Significantly more males were captured than females. Overall, significantly fewer adults were collected during treatment years (see first graph in Figure 18). Interactions among SEX x YEAR x FOREST and among SEX x YEAR TREATMENT were also significant $(\mathrm{P}=0.047$ and $\mathrm{P}=0.036$, respectively). For the first interaction, males were significantly less abundant in catches during the treatment years in the MON while the females were 
not significantly different in number. The second interaction showed significantly fewer males collected from $B t$ plots during the treatment years when compared to the $B t$ plots during pretreatment years, while females did not show a significant difference.

The second graph in Figure 18 illustrates the wing length measurements of moths collected during 1995 and 1998. A total of 1,052 intact males was measured. Females were not included in the ANOVA due to the low number of intact moths. The ANOVA indicated that males were significantly smaller during treatment years $(\mathrm{P}=0.005)$. A YEAR x FOREST x TREATMENT effect was also significant $(\mathrm{P}=0.025)$. The interaction was complex and has been illustrated in Figure 19. During treatment years in Ref plots, a significant difference in size was evident between forests, with MON moths smaller than GW moths. Differences were not significant for Bt plots. The GC plots showed a significant difference in the GW, with smaller moths collected during treatment years. The effect was not significant in the MON. A student's ttest conducted on wing lengths indicated that males were significantly smaller than females (15.711 and 17.090mm, respectively; $\mathrm{P}<0.001)$. 


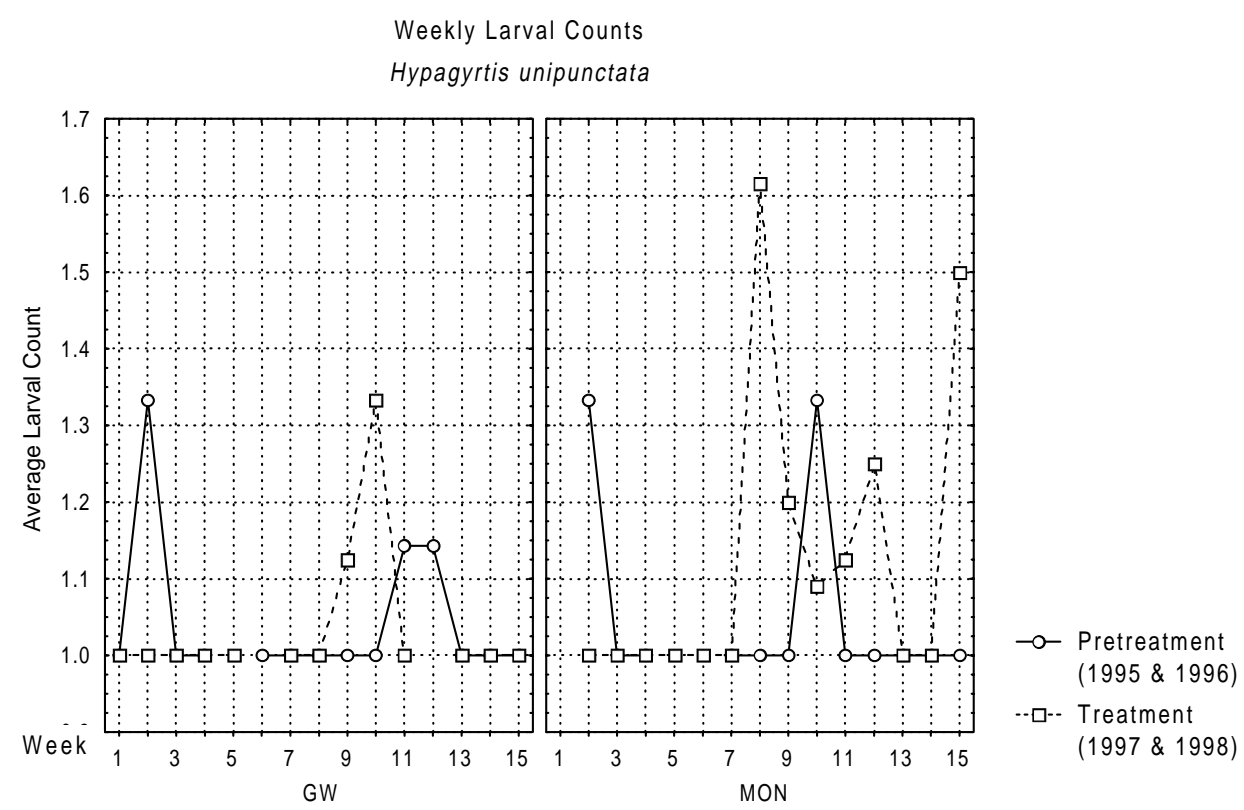

Weekly Adult Counts

Hypagyrtis unipunctata
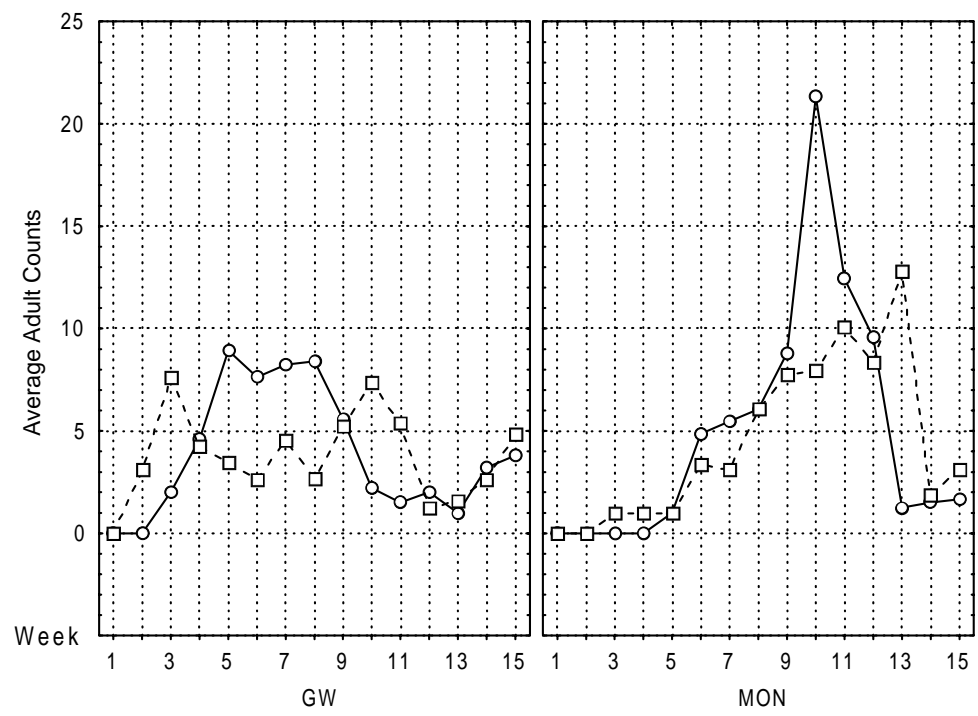

-o- Pretreatment (1995 \& 1996)

-.-- Treatment

(1997 \& 1998)

Figure 16. Weekly larval and adult counts (1995-1998). Graphs illustrate average weekly larval and adult counts of Hypagyrtis unipunctata collected during pretreatment and treatment years from the George Washington (GW) and Monongahela (MON) National Forests. 

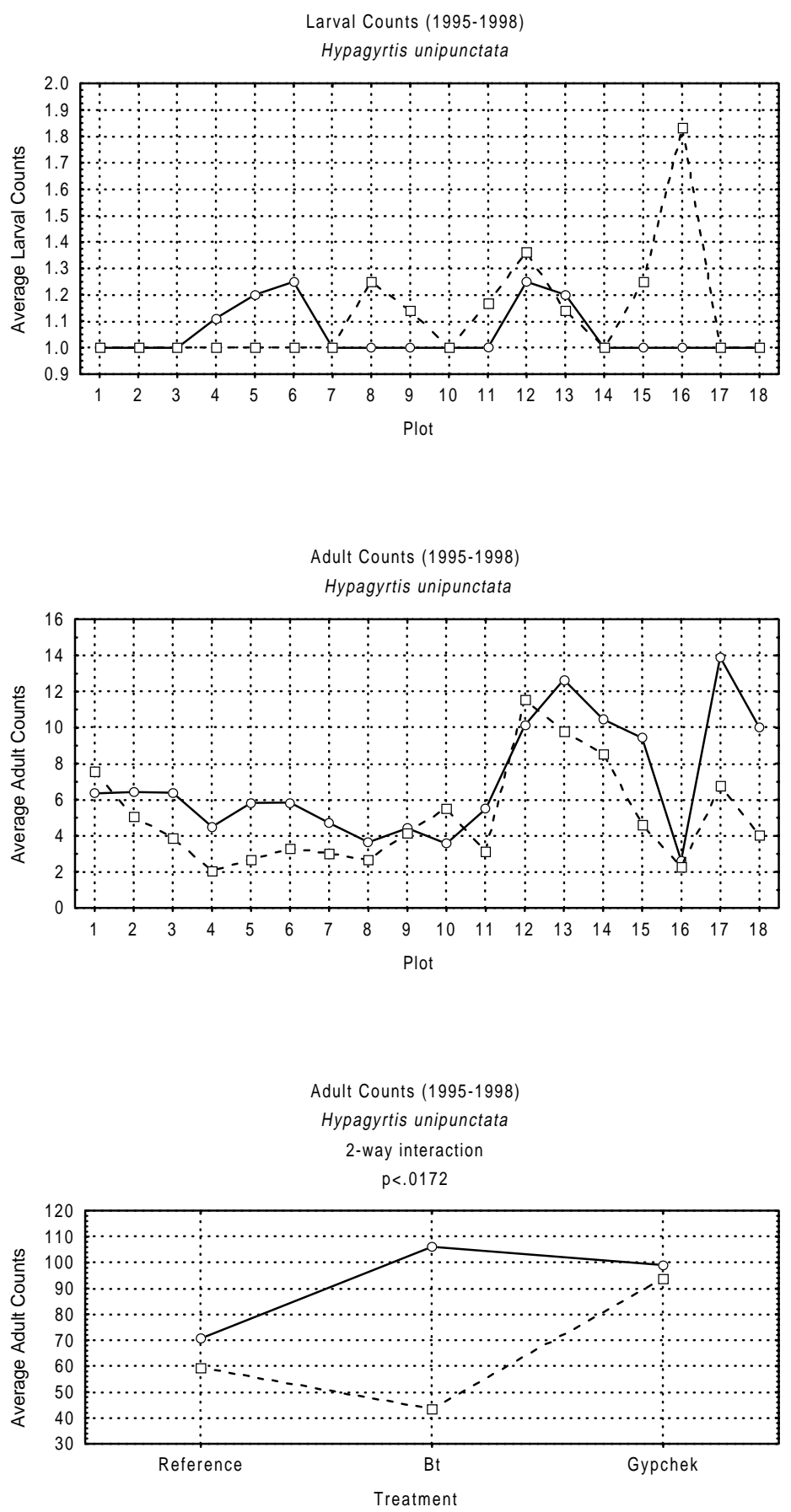

Reference:

(plots 2, 4, 8, 12, 14, 16)

Bt:

(plots 3, 5, 7, 11, 15, 18)

Gypchek:

(plots 1, 6, 9, 10, 13, 17)

- Pretreatment (1995 \& 1996)

$-\square$ - Treatment (1997 \& 1998)

Reference:

(plots 2, 4, 8, 12, 14, 16)

Bt:

(plots 3, 5, 7, 11, 15, 18)

Gypchek:

(plots 1, 6, 9, 10,13,17)

- - Pretreatment (1995 \& 1996)

- $\square$ - Treatment

(1997 \& 1998)

Figure 17. Larval and adult counts with adult interaction (1995-1998). The first two graphs illustrate average plot counts of larval and adult Hypagyrtis unipunctata collected during pretreatment and treatment years from the George Washington (plots 1-9) and Monongahela (plots 10-18) National Forests. The third graph illustrates an adult count interaction between treatment categories and pretreatment/treatment years. 


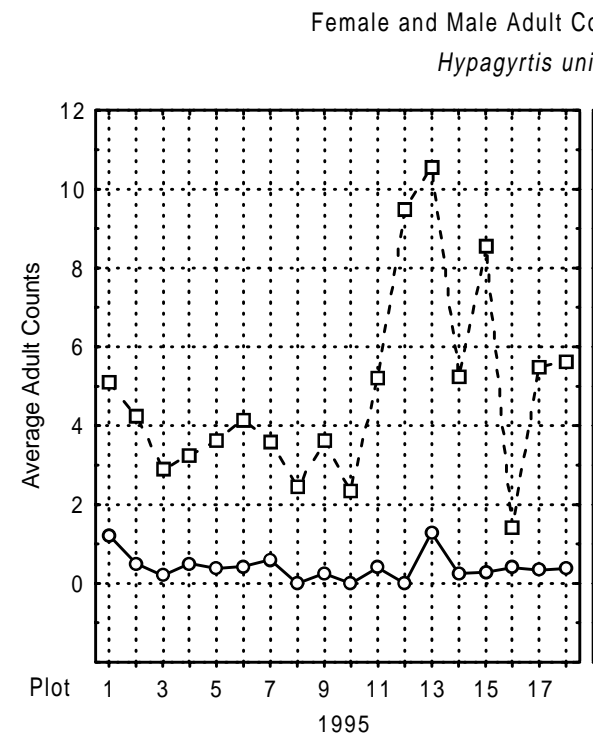

unts (1995 \& 1998)

punctata

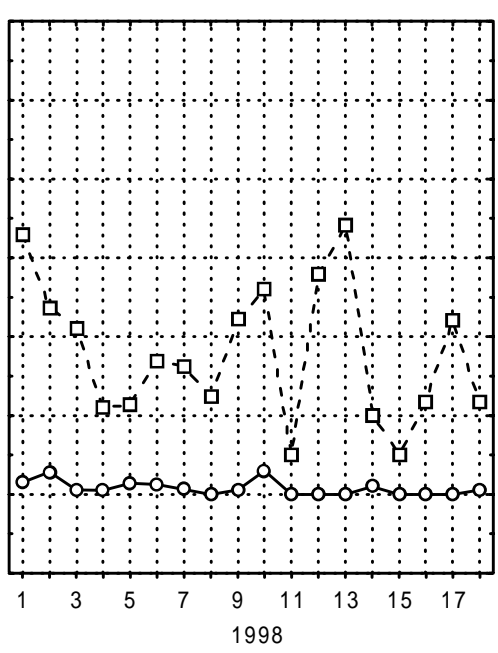

Reference:

(plots 2, 4, 8, 12, 14, 16)

$B t$ :

(plots 3, 5, 7, 11, 15, 18)

Gypchek:

(plots 1, 6, 9, 10, 13, 17)

-o- Females

- $\square$ - Males

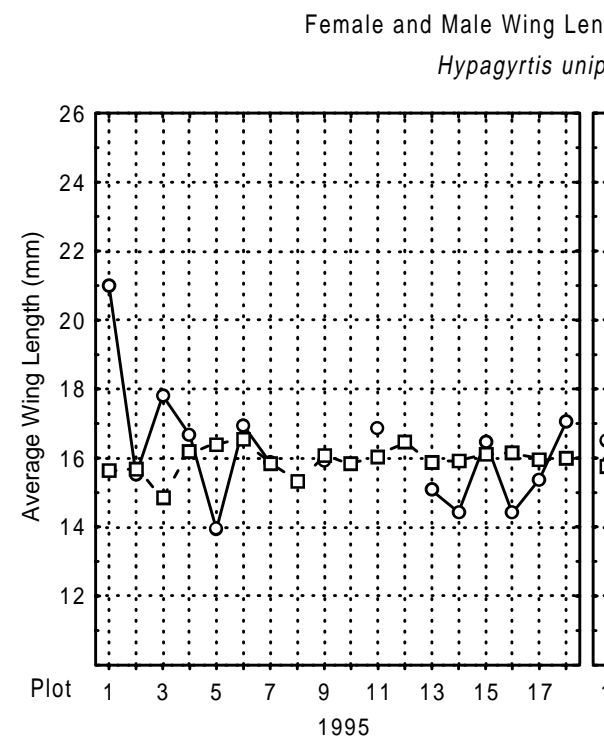

Hypagyrtis unipunctata

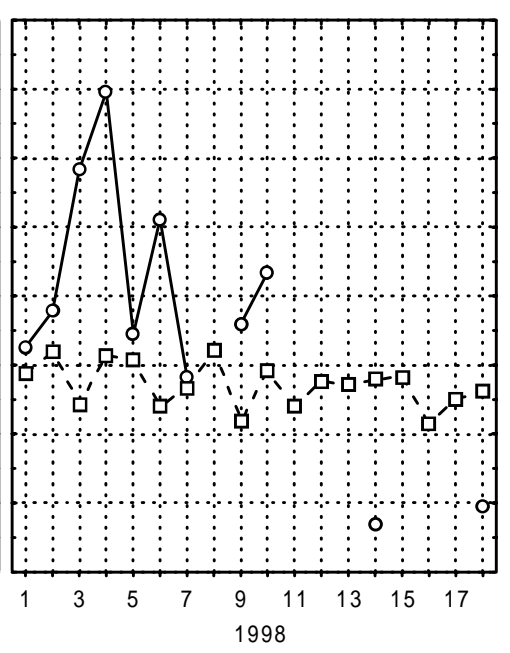

Reference:

(plots 2, 4, 8, 12, 14, 16)

$B t$ :

(plots 3, 5, 7, 11, 15, 18)

Gypchek:

(plots 1, 6, 9, 10, 13, 17)

-o- Females

- $\square$ - Males

Figure 18. Female and male adult counts and wing lengths (1995 \& 1998). Graphs illustrate average plot counts and wing lengths of male and female Hypagyrtis unipunctata collected during the 1995 pretreatment and 1998 treatment years from the George Washington (plots 1-9) and Monongahela (plots 10-18) National Forests. 

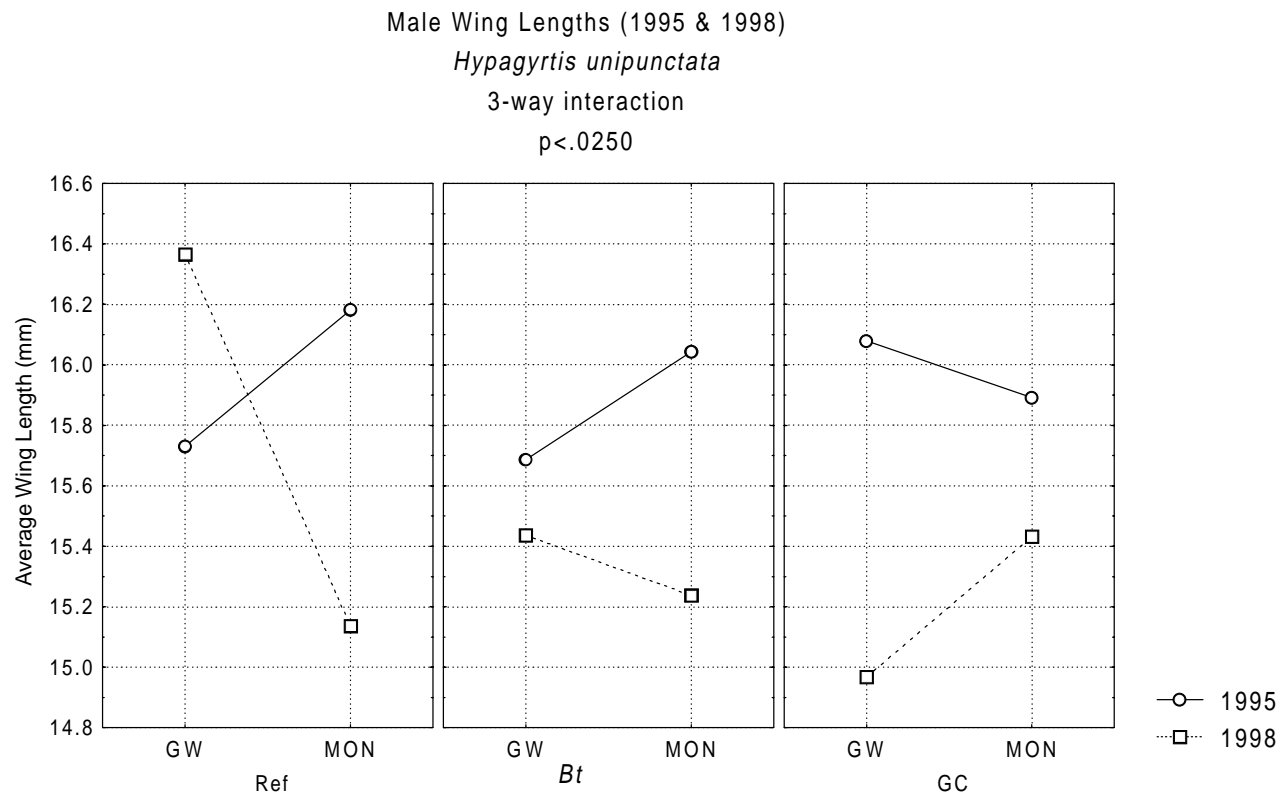

Figure 19. Male wing length interaction $(1995 \&$ 1998). The graph illustrates complex interaction among treatment categories for male Hypagyrtis unipunctata moths collected from the George Washington National Forest (GW) and the Monongahela National Forest (MON) during the 1995 pretreatment and 1998 treatment years.

\section{Lambdina fervidaria}

A total of 165 larvae and 11,015 adults was collected during 1995-1998. The bulk of larvae collected during pretreatment years from the GW was during weeks 6 and 1l. The MON larvae evidenced no peaks in catches during pretreatment years. Larvae collected weekly during treatment years failed to peak in the GW; however, weeks 9-15 showed higher numbers of larvae collected from the MON (see the first graph in Figure 20). Adults showed no major weekly peak in abundance in catches collected from the GW during pretreatment or treatment years. The MON, however, showed that the bulk of adults were collected during weeks 2-11 (see the second graph in

Figure 20).

The 3-way ANOVA for larval counts indicated significant effects for YEAR, FOREST, and YEAR x FOREST $(\mathrm{P}=0.003, \mathrm{P}=0.023$, and $\mathrm{P}=0.009$, respectively). The FOREST effect showed higher larval numbers during the treatment years. The treatment effect showed that a significant difference existed 
between $B t$ plots and Ref plots. The interaction showed that the 1998 counts were significantly higher in the Ref plots than in all treatment groups except the 1998 GC plots. During 1998, Bt plots were significantly reduced in larval number when compared to Ref and GC plots. This difference is obscured when 1995 data are included, as all plot groups from 1995 were not significantly different from the $1998 \mathrm{Bt}$ plots. The first and second graphs in Figure 21 illustrate general trends, the third graph illustrates the interaction.

The 3-way ANOVA for the adults indicated significant differences for YEAR, FOREST, and YEAR $\mathrm{x}$ FOREST $(\mathrm{P}=0.007, \mathrm{P}<0.001$, and $\mathrm{P}=0.002$, respectively). The YEAR effect shows significantly more moths collected during treatment years while the FOREST effect shows more moths collected from the MON than from the GW. The interaction indicated that significantly more moths were collected from the MON during 1998 than during 1995, as well as from either year in the GW.

During 1995 and 1998, 1,849 females and 3,718 male adult moths were identified. The 4-way ANOVA indicated that significant differences existed for SEX, YEAR, FOREST, SEX x FOREST, and YEAR x FOREST $(\mathrm{P}=0.015, \mathrm{P}=0.009, \mathrm{P}=0.002, \mathrm{P}=0.026$, and $\mathrm{P}=0.006$, respectively). Significantly more males were collected than females, more individuals were collected during treatment years, and more individuals were collected from the MON than from the GW. The SEX x FOREST interaction indicated that significantly more males were collected from the MON than any other statistical category. The YEAR $\mathrm{x}$ FOREST interaction indicated that during 1998, the number of individuals collected from the MON was significantly greater than any other statistical category (see the first graph in Figure 22).

Of the moths collected during 1995 and 1998, 1,788 females and 3,060 males were measured. Average wing lengths for both sexes are included in the second graph in Figure 22. Females were included in the 4-way ANOVA as their numbers were adequate. Significant differences in wing lengths were indicated for SEX, YEAR, FOREST, SEX x FOREST, YEAR x FOREST, and YEAR x FOREST x TREATMENT ( $\mathrm{P}=0.016, \mathrm{P}<0.001, \mathrm{P}<0.001, \mathrm{P}=0.004, \mathrm{P}=0.002$, and $\mathrm{P}=0.029$, respectively). For main effects, females were longer in wing length than males, treatment year moths were larger than pretreatment 
year moths, and moths collected from the MON were larger than those collected from the GW. The SEX $\mathrm{x}$ FOREST interaction shows that males collected from the GW were significantly smaller than all other statistical categories. The YEAR x FOREST interaction shows that the 1995 moths collected from the GW were significantly smaller than all other groups. The YEAR x FOREST x TREATMENT effects are complex and an illustration is provided as Figure 23. Essentially, 1995 moths collected from the $B t$ plots in the GW were smaller than all other groups, while moths collected from the same plots were larger than all other groups during 1998. Moths collected from the MON Bt plots during 1998 were the only moths to show a smaller size compared to the GW Bt plots during 1998. 

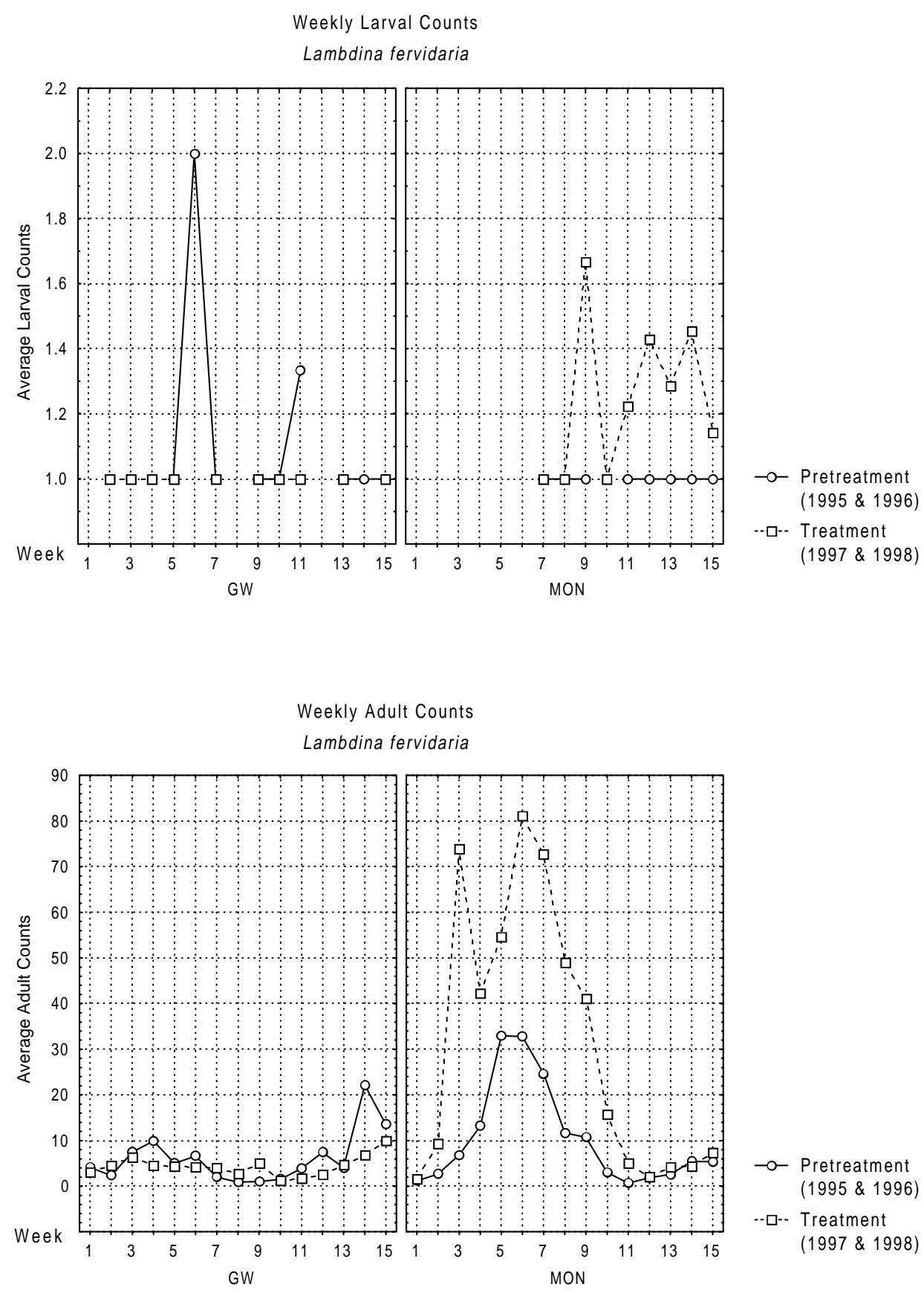

Figure 20. Weekly larval and adult counts (1995-1998). Graphs illustrate average weekly larval and adult counts of Lambdina fervidaria collected during pretreatment and treatment years from the George Washington (GW) and Monongahela (MON) National Forests. 

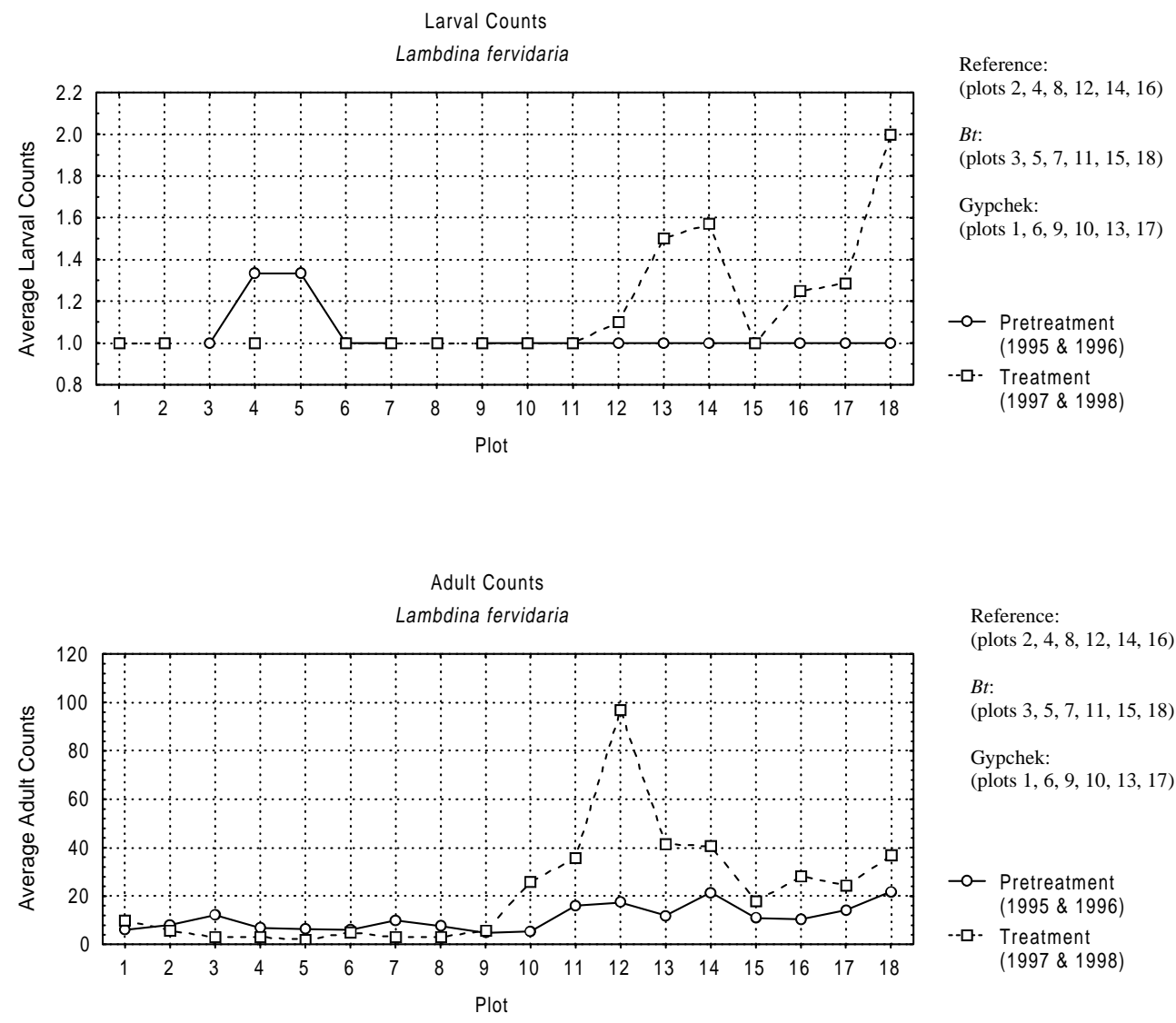

Reference:

(plots $2,4,8,12,14,16$ )

$B t$ :

(plots 3, 5, 7, 11, 15, 18)

Gypchek:

plots $1,6,9,10,13,17)$

-o- Pretreatment

(1995 \& 1996)

- $\square$ - Treatment

(1997 \& 1998)

Larval Counts

Lambdina fervidaria

2-way interaction

$p<.0094$

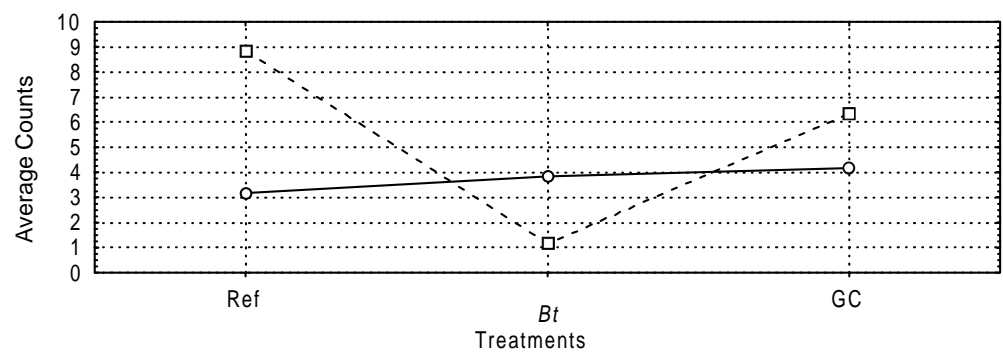

Figure 21. Larval and adult counts with larval interaction (1995-1998). The first two graphs illustrate average plot counts for larvae and adult Lambdina fervidaria collected during pretreatment and treatment years from the George Washington (plots 1-9) and Monongahela (plots 10-18) National Forests. The third graph illustrates the larval count interaction between treatment categories and pretreatment/treatment years. 


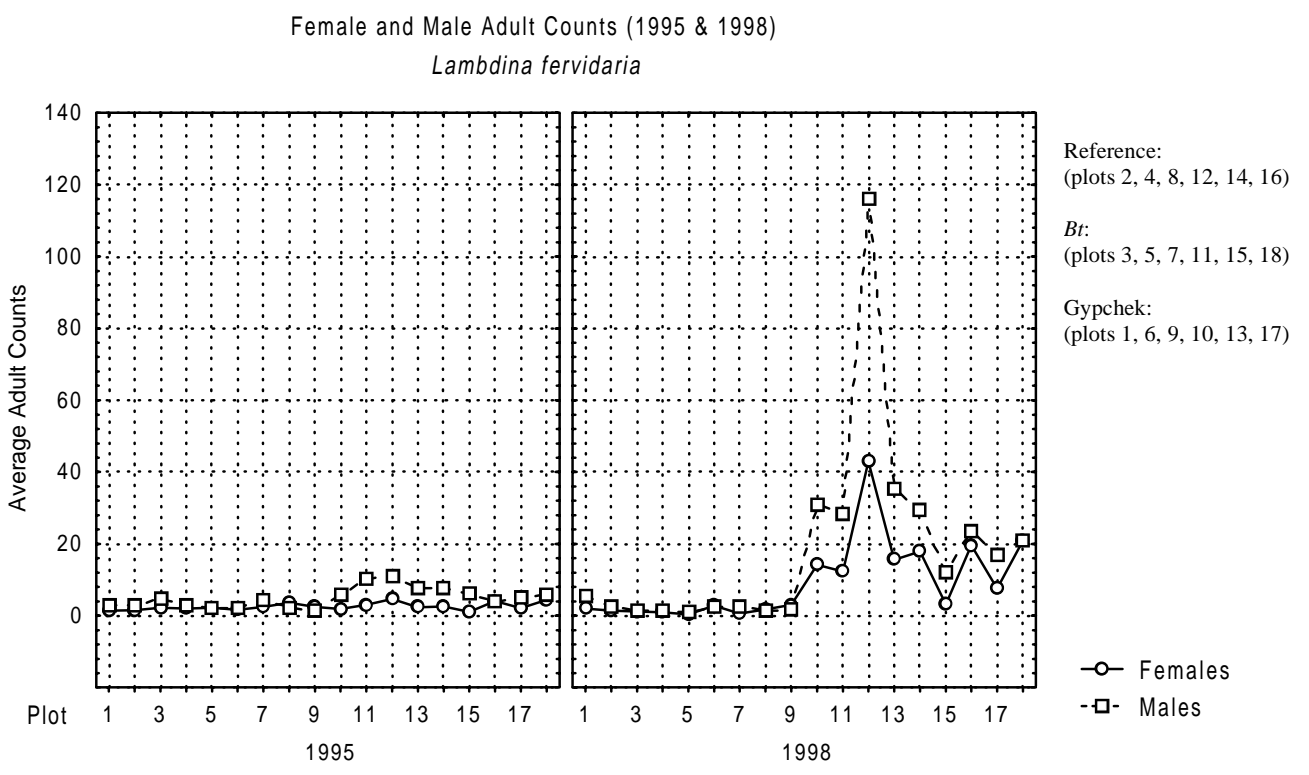

Female and Male Wing Lengths (1995 \& 1998)

Lambdina fervidaria
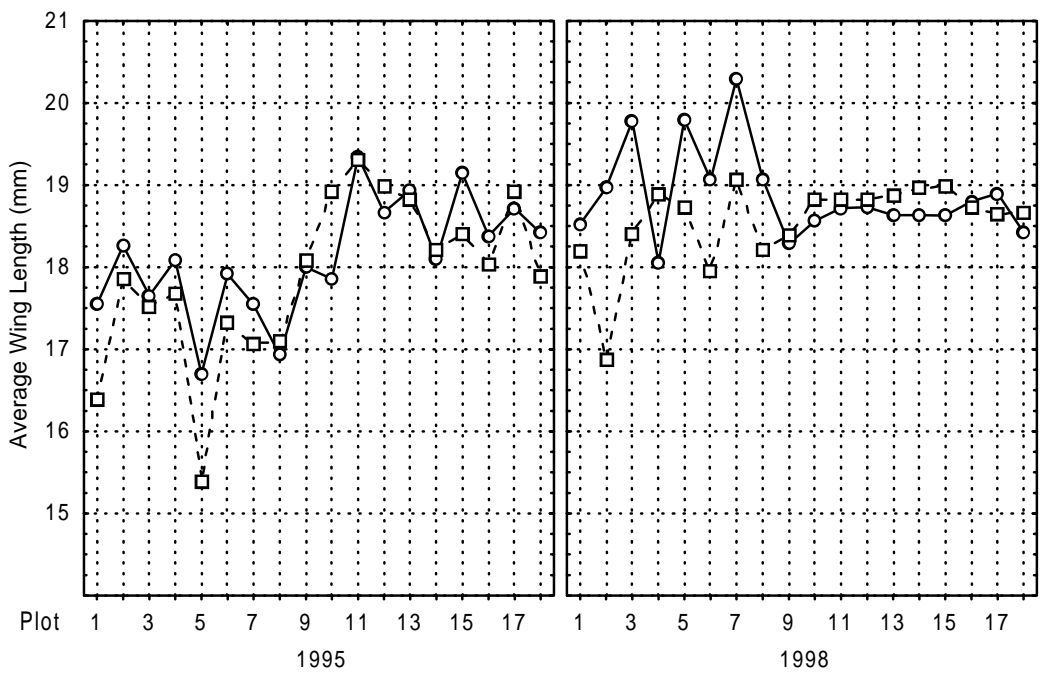

Reference:

(plots 2, 4, 8, 12, 14, 16)

$B t$ :

(plots $3,5,7,11,15,18)$

Gypchek:

(plots $1,6,9,10,13,17)$

-o- Females

- - Males

Figure 22. Female and male adult counts and wing lengths (1995 \& 1998). Graphs illustrate female and male adult plot counts and wing lengths of Lambdina fervidaria collected during the 1995 pretreatment and 1998 treatment years from the George Washington (plots 1-9) and Monongahela (plots 10-18) National Forests. 

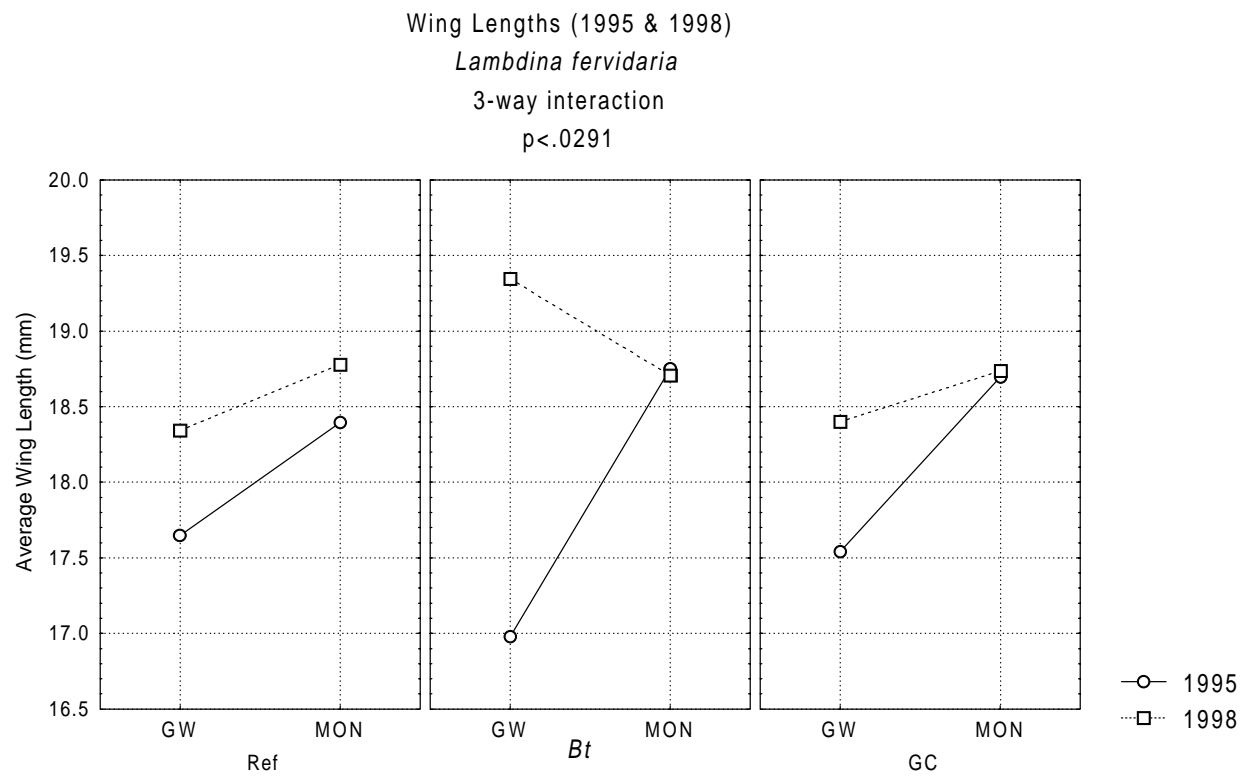

Figure 23. Wing length interaction $(1995 \&$ 1998). The graph illustrates the complex interaction of Lambdina fervidaria wing lengths among treatment categories in the George Washington (GW) and Monongahela (MON) National Forests during the 1995 pretreatment and 1998 treatment years.

\section{Campaea perlata}

A total of 176 larvae and 2,455 adults was collected during 1995-1998. The weekly larval catches for pretreatment and treatment years in both forests appeared to occur primarily between weeks 6-15. Peak larval catch occurred slightly earlier during treatment years on the Mon (see first graph in Figure 24). The bulk of adults were collected between weeks 3-10 from both forests during pretreatment and treatment years (see second graph in Figure 24).

An ANOVA for larvae showed no significant effects. However, the ANOVA for adults indicated a significant effect for YEAR ( $\mathrm{P}=0.023)$. More moths were collected during the treatment years than during pretreatment years. The first and second graph in Figure 25 illustrate the general trends for larvae and adults, respectively.

A total of 176 female and 1,449 male adult moths was identified from 1995 and 1998 catches. The ANOVA indicated that the SEX effect was indeed significant $(\mathrm{P}=0.003)$. Interactions between SEX $\mathrm{x}$ 
YEAR and SEX x FOREST ( $\mathrm{P}=0.039$ and $\mathrm{P}=0.047$, respectively) were also detected. Significantly more male moths were collected during treatment years than each of the other statistical categories. Male moths were collected in significantly more numbers from the MON (see the first graph in Figure 26).

A total of 146 female and 1,233 intact moths was measured for wing length. The 4-way ANOVA indicated that females were significantly longer in wing length than males. The second graph in Figure 26 illustrates the trend. 

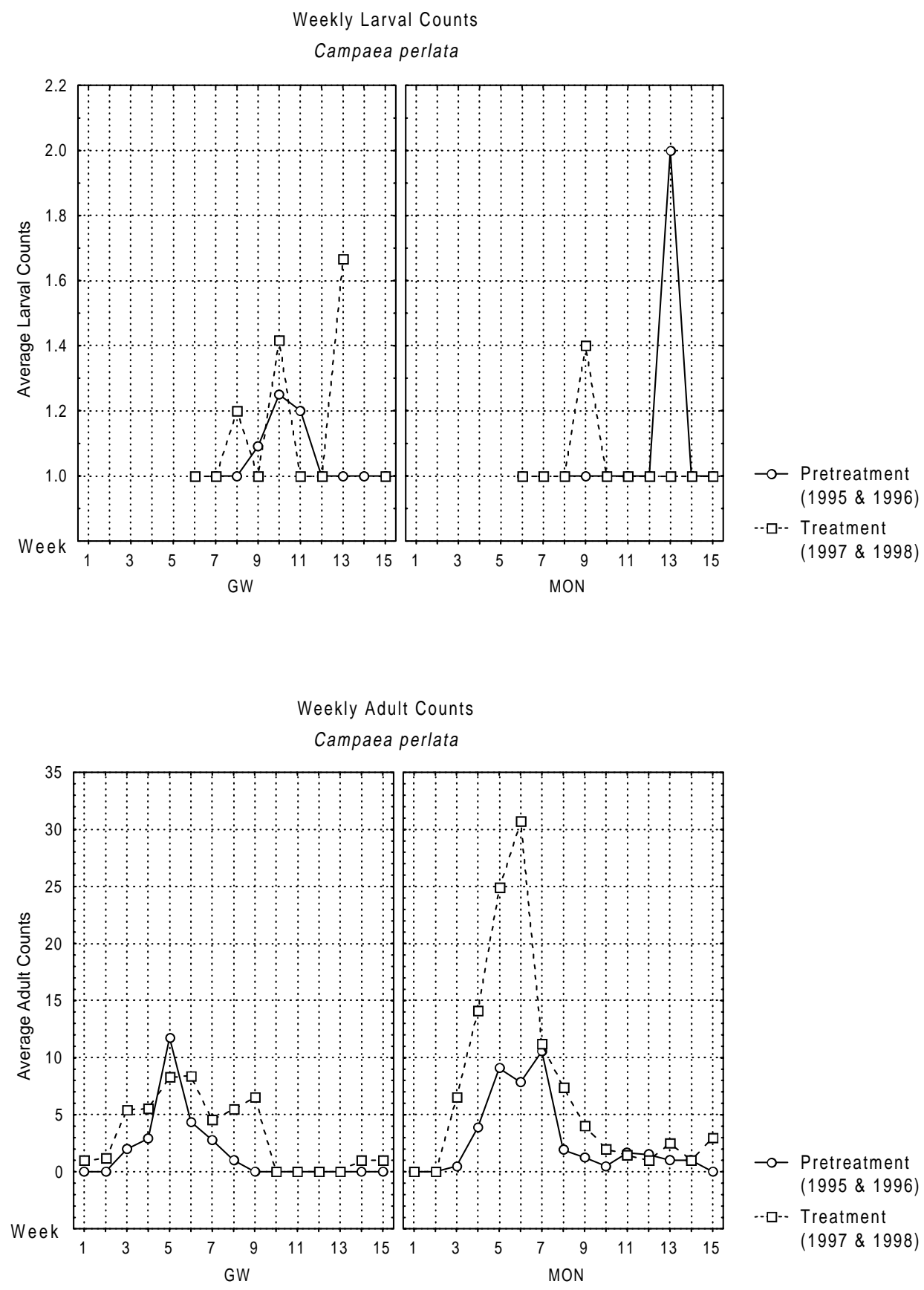

Figure 24. Weekly larval and adult counts (1995-1998). Graphs illustrate average weekly larval and adult counts of Campaea perlata collected during pretreatment and treatment years from the George Washington (GW) and Monongahela (MON) National Forests. 


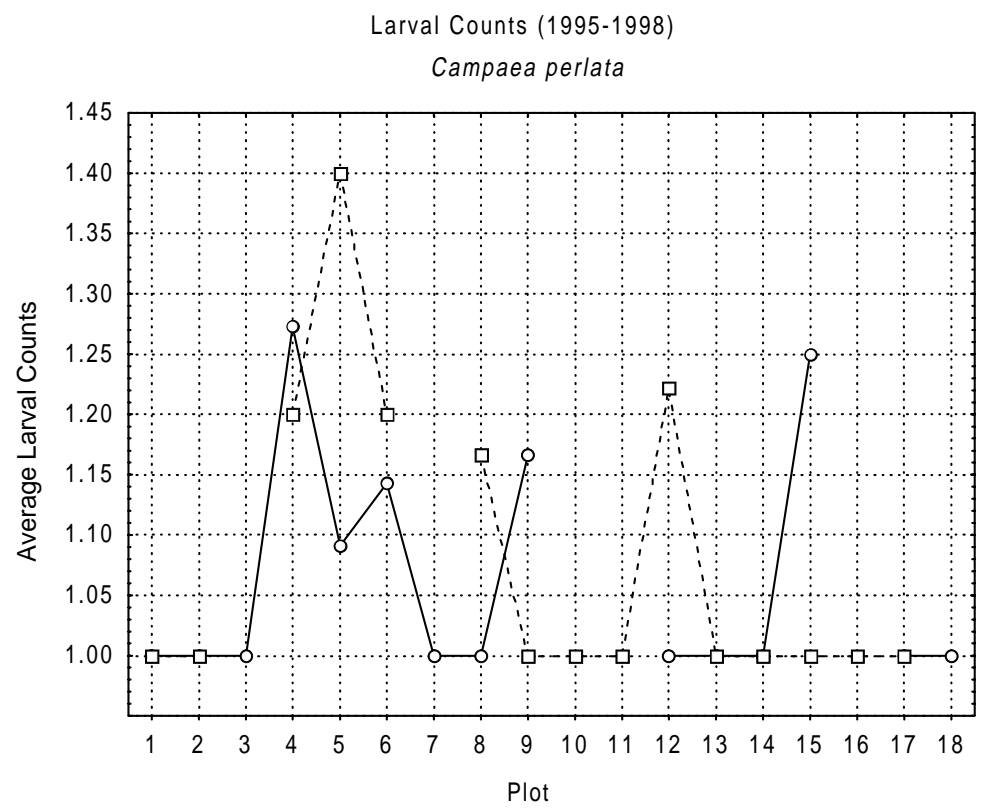

Reference:

(plots 2, 4, 8, 12, 14, 16)

$B t$ :

(plots 3, 5, 7, 11, 15, 18)

Gypchek:

(plots 1, 6, 9, 10, 13, 17)

- - Pretreatment

(1995 \& 1996)

-- -- Treatment

(1997 \& 1998)

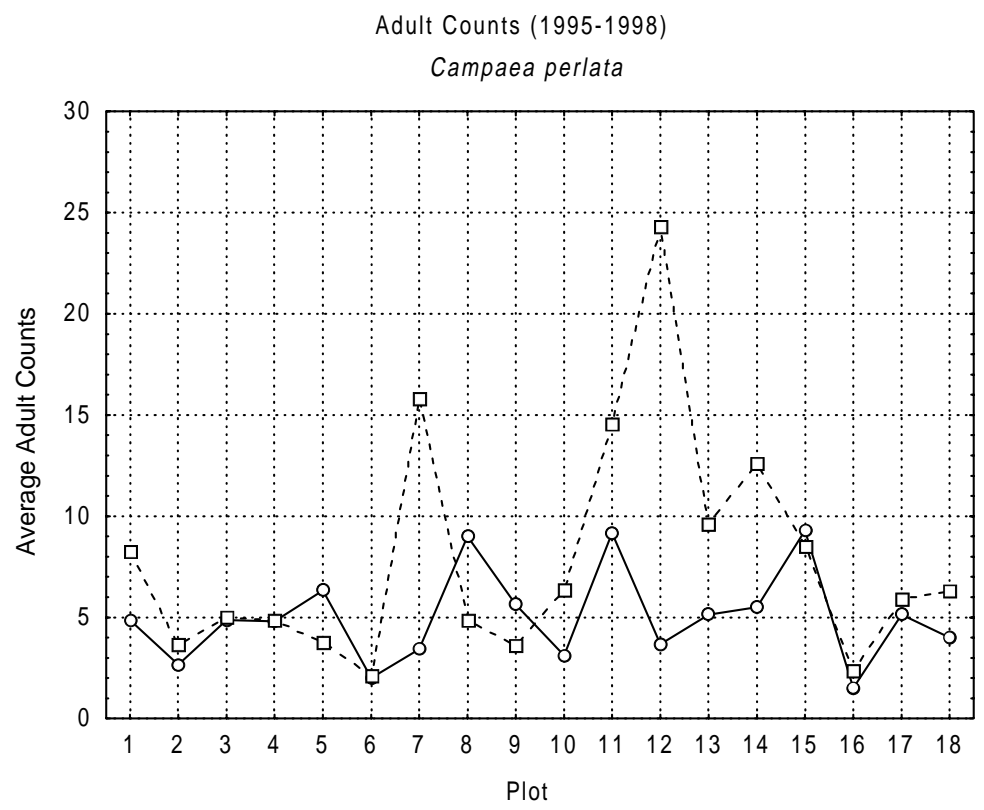

Reference:

(plots 2, 4, 8, 12, 14, 16)

Bt:

(plots 3, 5, 7, 11, 15, 18)

Gypchek:

(plots 1, 6, 9, 10, 13, 17)

- - Pretreatment

(1995 \& 1996)

-- -- Treatment

(1997 \& 1998)

Figure 25. Larval and adult counts (1995-1998). Graphs illustrate average larval and adult plot counts of Campaea perlata collected during pretreatment and treatment years from the George Washington $(\mathrm{GW})$ and Monongahela (MON) National Forests. 

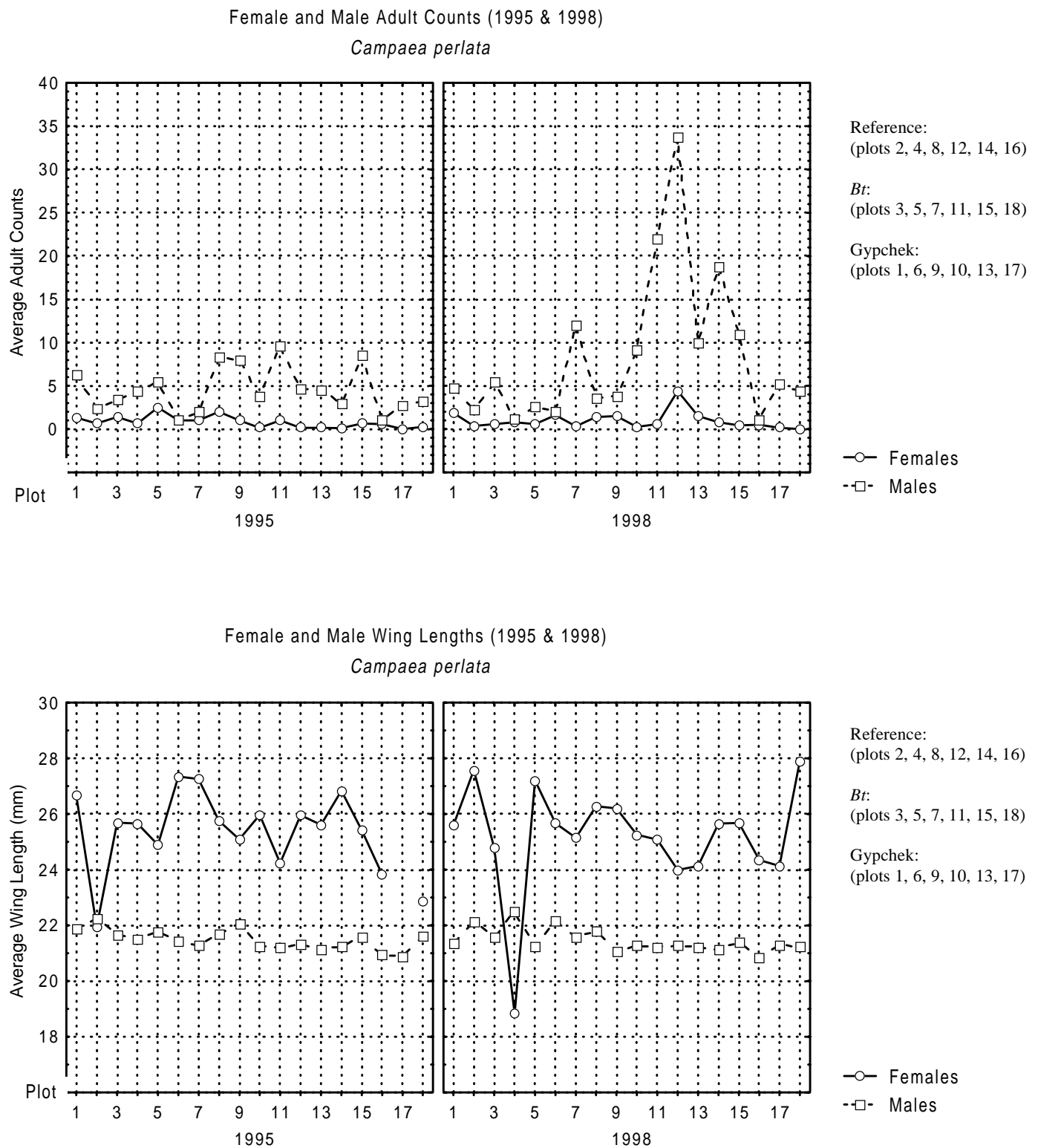

Figure 26. Female and male counts and wing lengths (1995 \& 1998). Graphs illustrate the average plot counts and wing lengths for Campaea perlata collected during the 1995 pretreatment and 1998 treatment years from the George Washington (GW) and Monongahela (MON) National Forests. 


\section{Tetracis cachexiata}

A total of 66 larvae and 1,742 adults was collected during 1995-1998. The first graph in Figure 27 illustrates the average weekly catches for larvae, while the second graph illustrates average weekly catches for adults. Larvae appear to peak late during the sampling season (weeks 12-15), while the adults peaked in catches within the first 9 weeks in both forests during treatment and pretreatment years.

A 3-way ANOVA conducted on larval data showed no significant effects. Adults, however, showed significant main effects for YEAR and for FOREST ( $\mathrm{P}=0.006$ and $\mathrm{P}=0.012$, respectively). More moths were collected during treatment years and more were collected from the MON than from the GW. General treads for larvae and adults are illustrated in the first and second graphs of Figure 28, respectively.

A total of 104 female and 926 male moths was identified from 1995 and 1998 catches. The SEX difference was significant $(\mathrm{P}=0.002)$. A YEAR effect $(\mathrm{P}=0.027)$ and a FOREST effect $(\mathrm{P}=0.034)$ were also significant. More moths were collected during treatment years than from pretreatment years, and more were collected from the MON than from the GW. Significant interactions were identified for SEX x YEAR and for SEX x FOREST $(\mathrm{P}=0.027$ and $\mathrm{P}=0.025$, respectively). Interactions indicated that males were more abundant in catches during 1998 in the MON. Trends are illustrated in the first graph in Figure 29.

A total of 113 intact female and 846 intact male moths were measured for wing length. The second graph in Figure 29 illustrates the general trends. Several gaps in the data were evident. However by substituting group means for these gaps, a 4-way ANOVA was conducted in an effort to show differences, though some accuracy may have been potentially sacrificed. Main effects were noted for SEX and for FOREST $(\mathrm{P}<0.001$ and $\mathrm{P}=0.019$, respectively). Females were longer on average in wing length (23.470 $\mathrm{mm})$ than males $(21.053 \mathrm{~mm})$. The $\mathrm{GW}$ moths were longer than MON moths (22.734 and $21.989 \mathrm{~mm})$, respectively. 

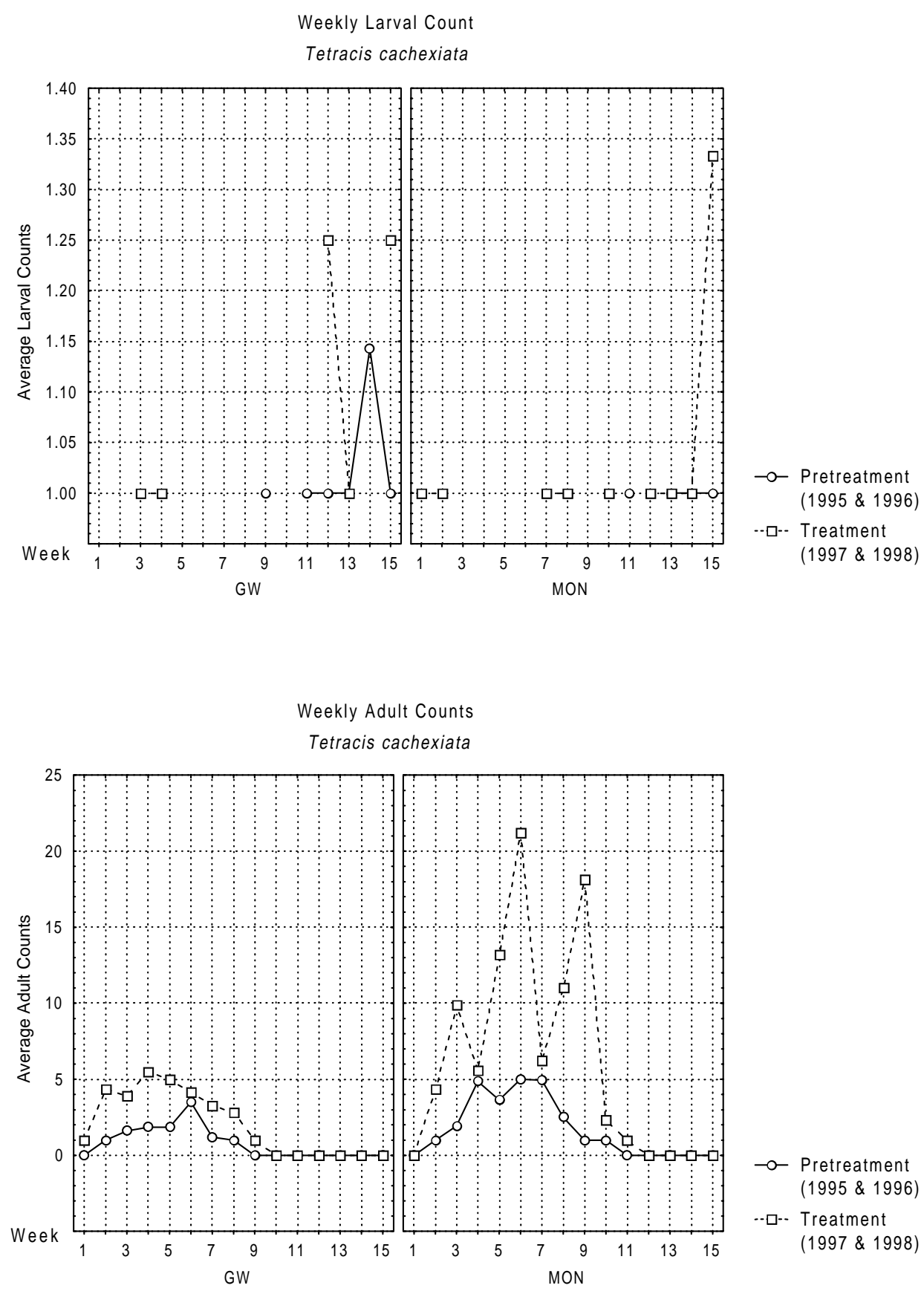

Figure 27. Weekly larval and adult counts (1995-1998). Graphs illustrate average weekly larval and adult counts of Tetracis cachexiata collected during pretreatment and treatment years from the George Washington (GW) and Monongahela (MON) National Forests. 


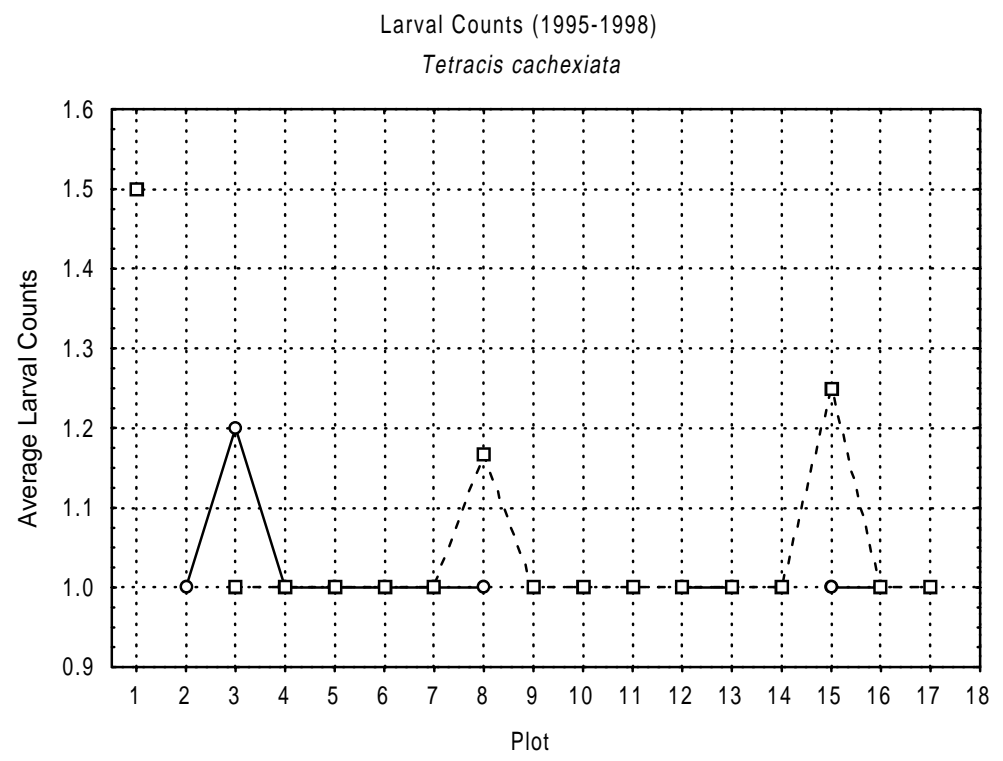

Reference

(plots 2, 4, 8, 12, 14, 16)

$B t$ :

(plots 3, 5, 7, 11, 15, 18)

Gypchek:

(plots $1,6,9,10,13,17$ )

- - Pretreatment

- $\square$ - Treatment

(1997 \& 1998)

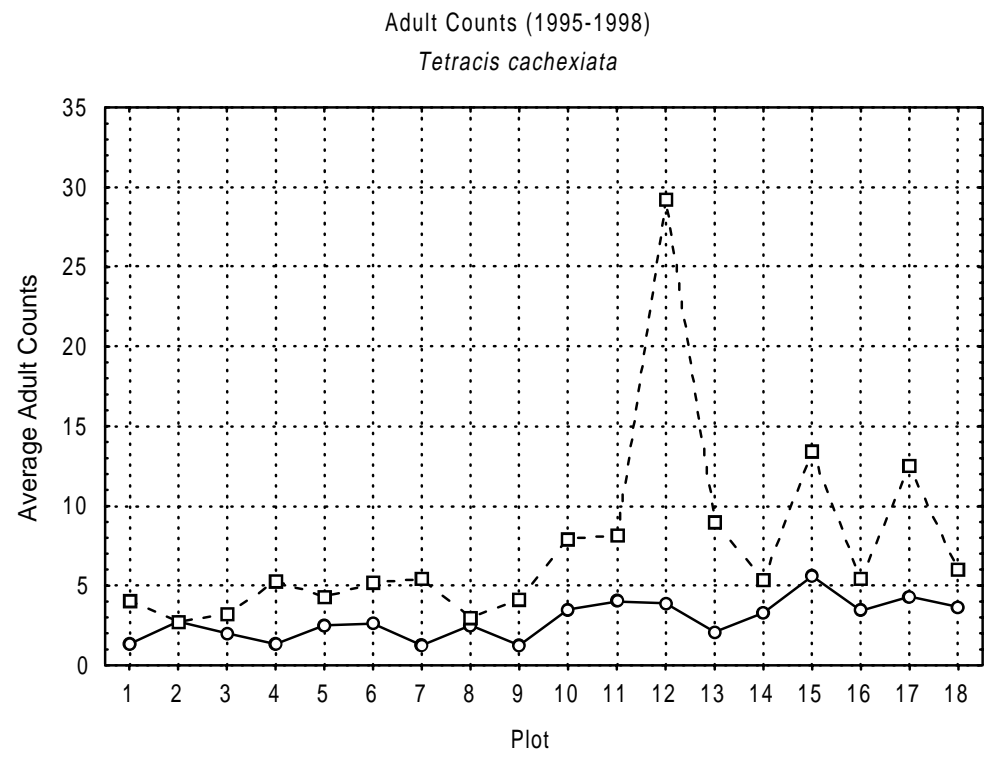

Reference:

(plots 2, 4, 8, 12, 14, 16)

$B t$ :

(plots $3,5,7,11,15,18)$

Gypchek:

(plots 1, 6, 9, 10, 13, 17)

$-\infty$ Pretreatment

(1995 \& 1996)

- $\square$ - Treatment (1997 \& 1998)

Figure 28. Larval and adult counts (1995-1998). Graphs illustrate average plot counts of larva and adult Tetracis cachexiata collected during pretreatment and treatment years from the George Washington (plots 1-9) and Monongahela (plots 10-18) National Forests. 

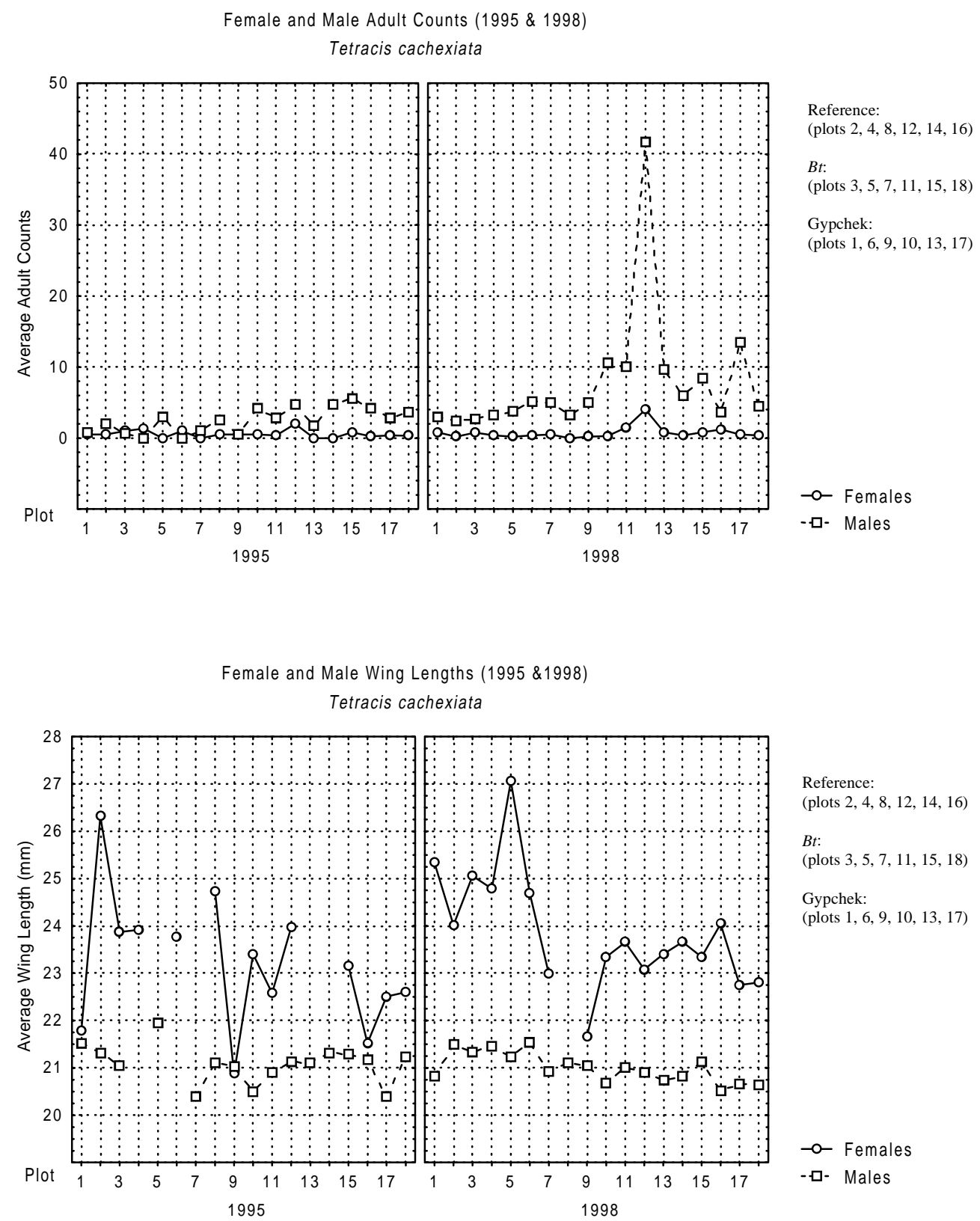

Figure 29. Female and male adult counts and wing lengths (1995 \& 1998). Graphs illustrate average plot counts and wing lengths of male and female Tetracis cachexiata collected during the 1995 pretreatment and 1998 treatment years from the George Washington (plots 1-9) and Monongahela (plots 10-18) National Forests. 


\section{Itame pustularia}

A total of 227 larvae and 6,147 adults was collected during 1995-1998. The first and second graphs in Figure 30 illustrate the average weekly catches of larvae and adults, respectively. Larvae show a peak in catches collected from the GW during pretreatment years between weeks 2-8. There is no peak evident during treatment years in the GW. The MON larvae, however, show variability throughout the sampling seasons for pretreatment and treatment years. Adults in the GW were most abundant during weeks 7-14. The MON adults were delayed about one week in comparison. Pretreatment and treatment years were similar for the adults.

Larval and adult counts relative to the plots are illustrated in the first and second graphs in Figure 31, respectively. The ANOVA for the larvae indicated a significant interaction for YEAR $\mathrm{x}$ FOREST ( $\mathrm{P}=0.008)$. In this case, there were significantly fewer larvae in the GW during 1998 than any other statistical category. Adults showed a significant main effect for YEAR ( $\mathrm{P}=0.023)$, with 1995 moths more abundant than 1998 moths. The YEAR x FOREST interaction was also significant $(\mathrm{P}=0.030)$, with significantly more moths in the GW during 1995 than any of the other categories.

A total of 1,716 female and 3,035 male moths was identified from 1995 and 1998. The effect for SEX was significant $(\mathrm{P}=0.046)$. Interactions for SEX x FOREST and YEAR x FOREST were significant $(\mathrm{P}=0.027$ and $\mathrm{P}=0.049$, respectively). Significantly more males were collected in the GW. During 1995, more moths were collected than during 1998. The trends are illustrated in the first graph in Figure 32.

The second graph in Figure 32 depicts wing length measurements for 1995 and 1998. A total of 1,303 females and 2,379 males were measured. Significant main effects for SEX and YEAR were indicated by the 4-way ANOVA ( $\mathrm{P}<0.001$ and $\mathrm{P}=0.008$, respectively). Females were significantly longer in wing length, and the wings of moths collected during 1998 were longer than those collected during 1995. The interaction for SEX x YEAR was also significant $(\mathrm{P}=0.010)$. Females, though larger than males in all instances, showed a significant increase in size from 1995 to 1998. 

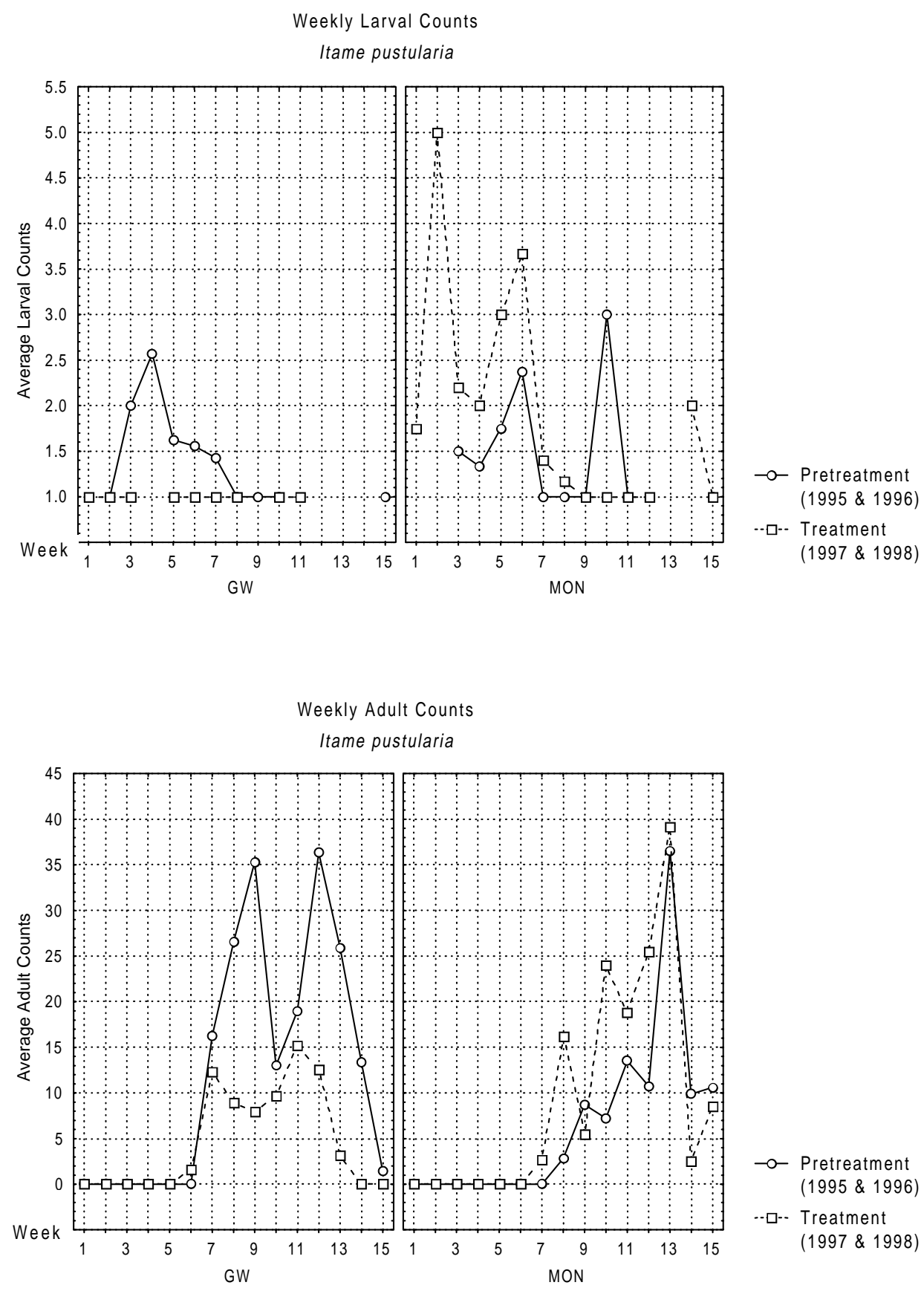

Figure 30. Weekly larval and adult counts (1995-1998). Graphs illustrate average weekly larval and adult counts of Itame pustularia collected during pretreatment and treatment years from the George Washington (GW) and Monongahela (MON) National Forests. 

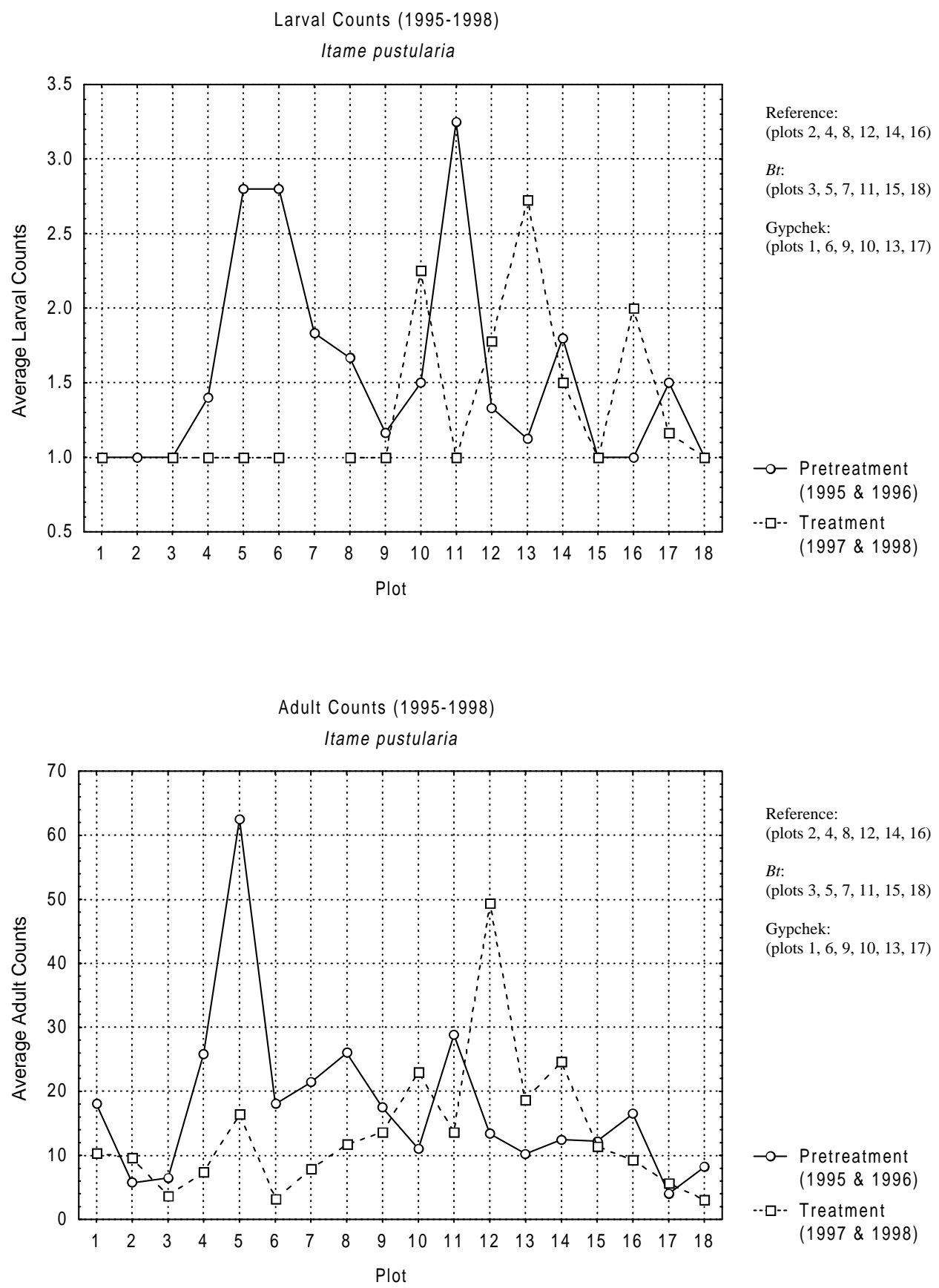

Figure 31. Larval and adult counts (1995-1998). Graphs illustrate average plot counts of Itame pustularia collected during pretreatment and treatment years from the George Washington (plots 1-9) and Monongahela (plots 10-18) National Forests. 
Female and Male Adult Counts (1995 \& 1998)

Itame pustularia
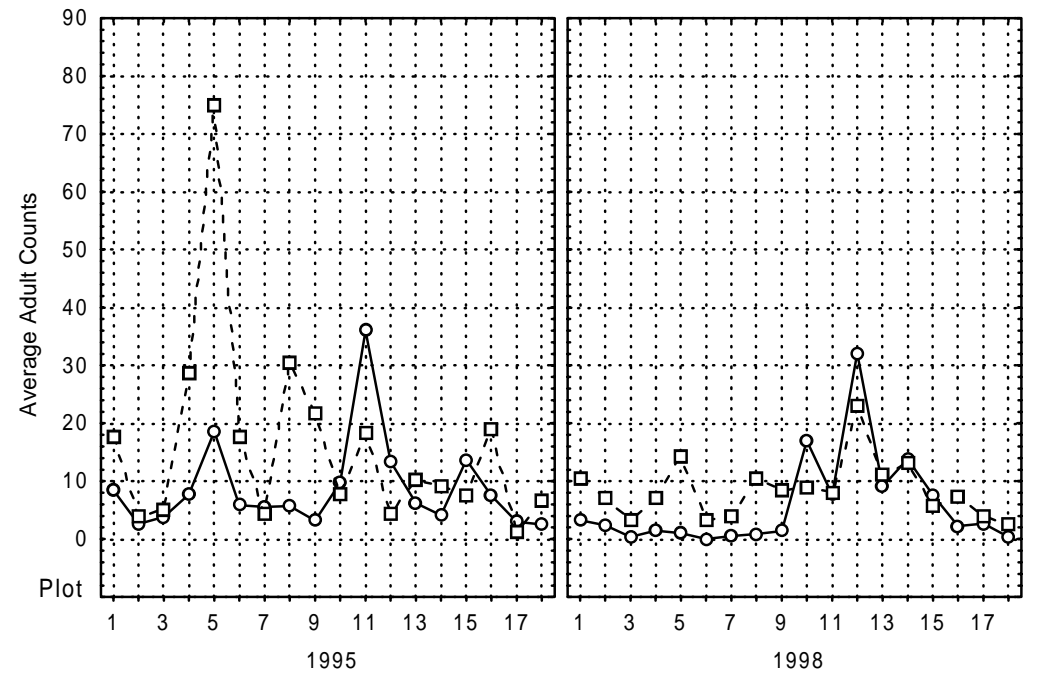

Female and Male Wing Lengths (1995 \& 1998)

Itame pustularia

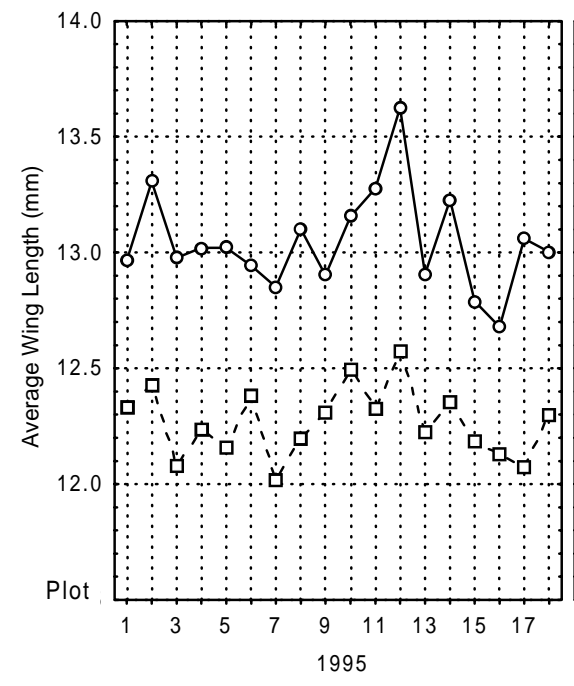

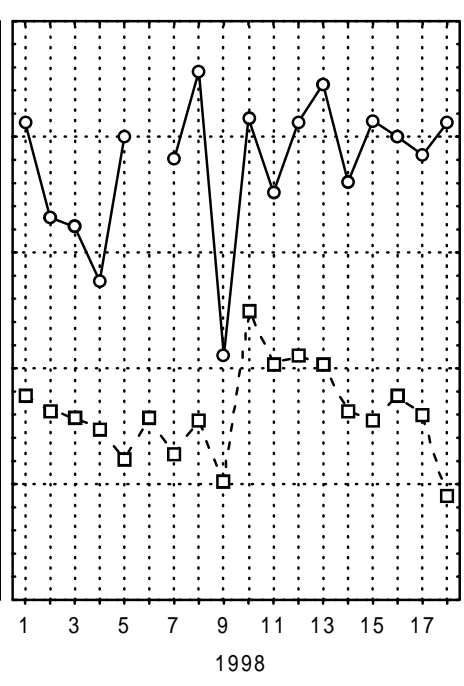

Reference:

(plots $2,4,8,12,14,16)$

(plots $3,5,7,11,15,18)$

Gypchek:

(plots $1,6,9,10,13,17$ )

-o- Females

--口- Males

Reference:

(plots $2,4,8,12,14,16)$

$B t$ :

(plots 3, 5, 7, 11, 15, 18)

Gypchek:

(plots $1,6,9,10,13,17$ )

-o- Females

---. Males

Figure 32. Female and male adult counts and wing lengths (1995 \& 1998). Graphs illustrate average plot counts and wing lengths of female and male Itame pustularia collected during the 1995 pretreatment and 1998 treatment years from the George Washington (plots 1-9) and Monongahela (plots 10-18) National Forests. 


\section{Besma quercivoraria}

A total of 198 larvae and 1,633 adults was collected during 1995-1998. The graphs in Figure 33 illustrate average weekly catches. Larvae showed an early and late season peak in the GW during pretreatment years which were not seen during treatment years. In the MON, a late season peak was demonstrated for both pretreatment and treatment years. Adults also showed early and late season peaks. Both forests showed similar peaks during pretreatment and treatment years.

Larval and adult counts relative to the plots are illustrated in the first and second graphs in Figure 34, respectively. The ANOVA indicated that significantly fewer numbers of larvae and adults were collected during treatment years $(\mathrm{P}=0.002$ and $\mathrm{P}=0.011$, respectively). There was also significant interaction for YEAR x FOREST for adults $(\mathrm{P}=0.014)$. There were significantly more moths collected from the MON than all other statistical categories during pretreatment years.

A total of 90 female and 594 male adults were identified from 1995 and 1998 catches (see the first graph presented in Figure 35). The 4-way ANOVA indicated significant main effects for SEX and YEAR $(\mathrm{P}<0.001$ and $\mathrm{P}=0.001$, respectively). More males were captured than females and more moths were collected during 1995 than during 1998. Several interactions were also significant for SEX x YEAR, SEX x FOREST, and YEAR x FOREST, with $\mathrm{P}=0.012, \mathrm{P}=0.050$, and $\mathrm{P}=0.008$, respectively. The 3-way interaction indicated that the male moths from the MON during 1995 were significantly more abundant in the catches than from any other category. The second graph in Figure 35 illustrates that wing length data for females were incomplete for 4-way ANOVA due to insufficient numbers for all statistical categories. When males were analyzed alone, significant differences were not identified. 

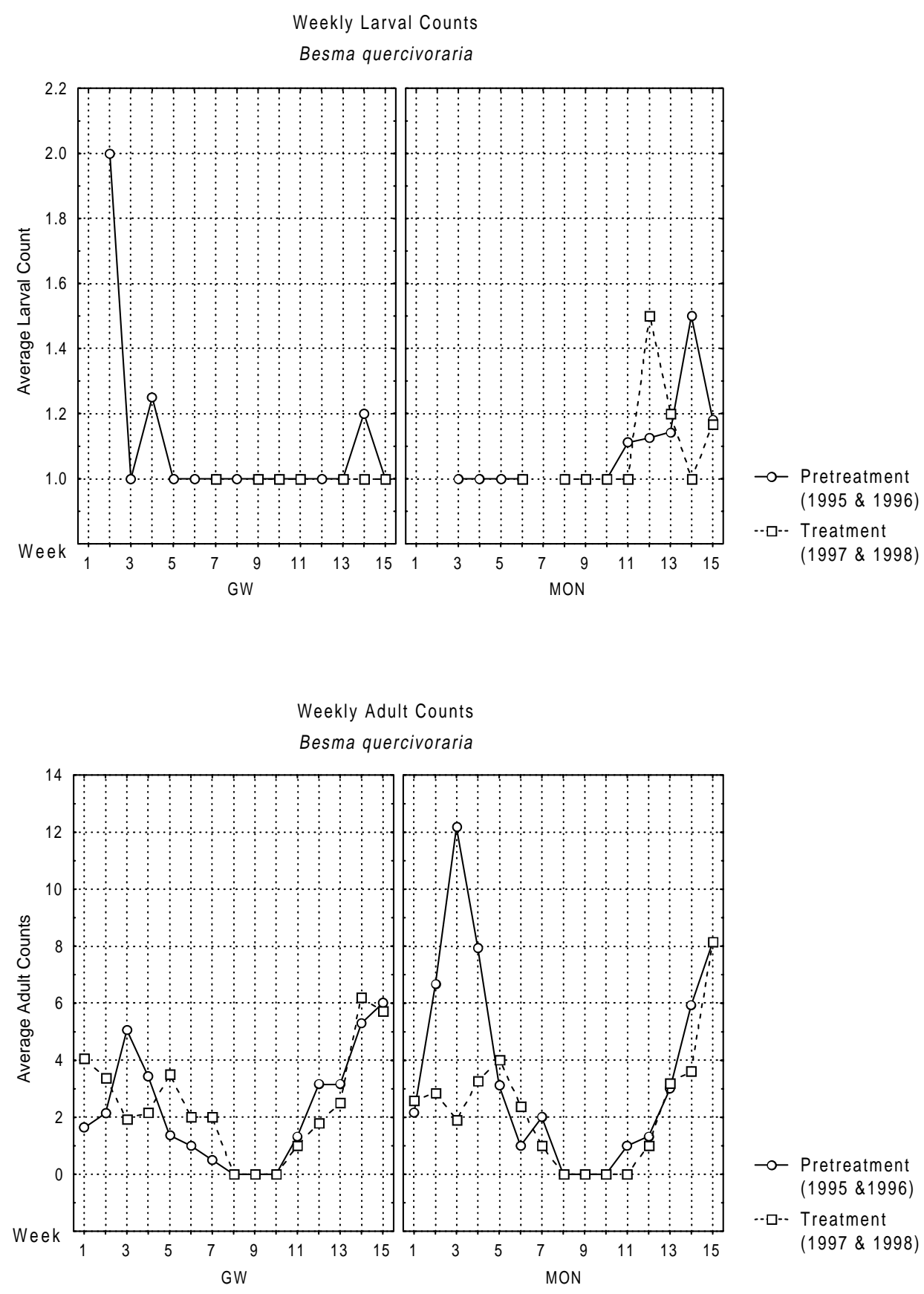

Figure 33. Weekly larval and adult counts (1995-1998). Graphs illustrate average weekly larval and adult counts of Besma quercivoraria collected during pretreatment and treatment years from the George Washington (GW) and Monongahela (MON) National Forests. 

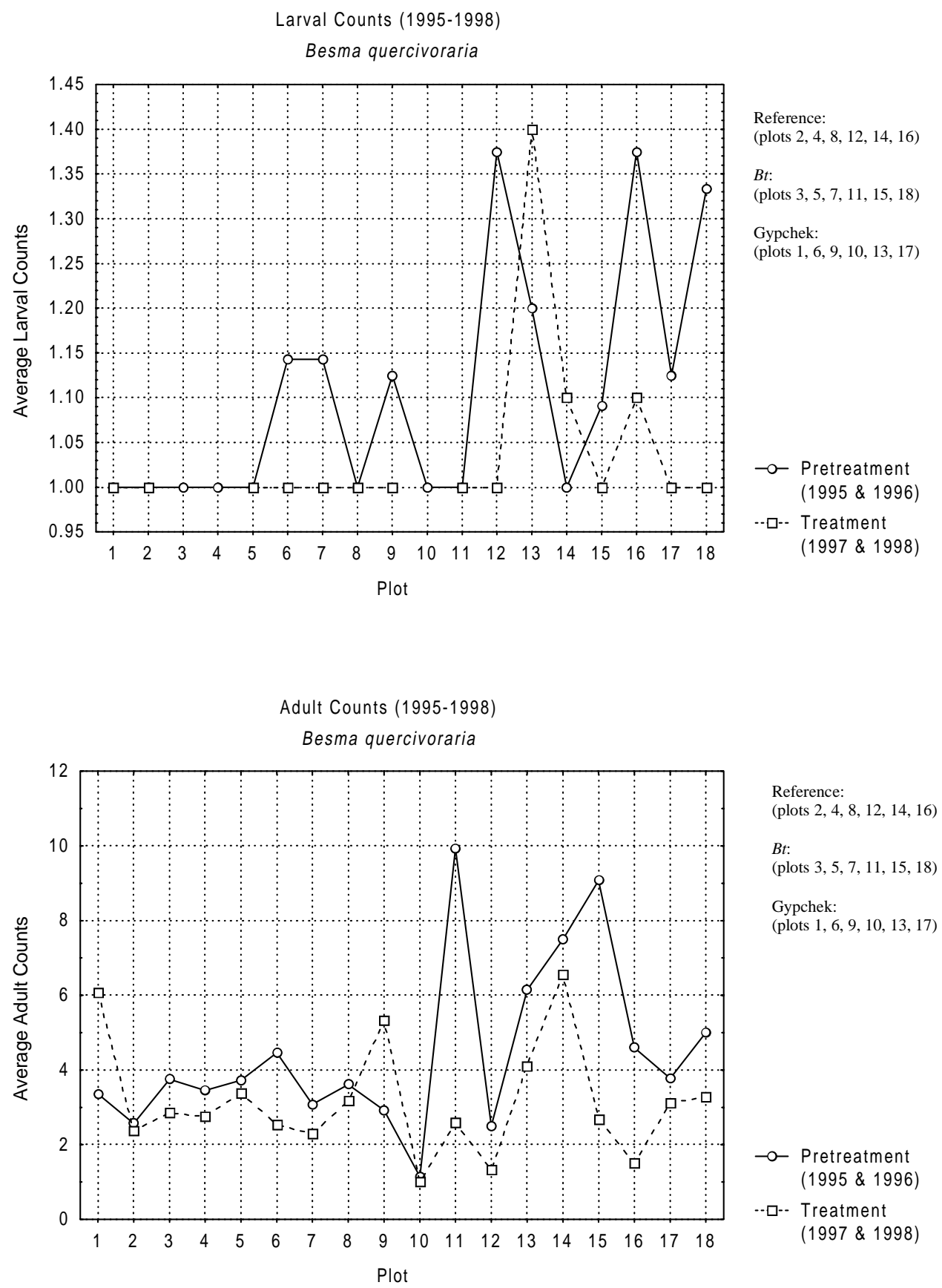

Figure 34. Larval and adult counts (1995-1998). Graphs illustrate average plot counts of larval and adult Besma quercivoraria collected during pretreatment and treatment years from the George Washington (plots 1-9) and Monongahela (plots 10-18) National Forests. 

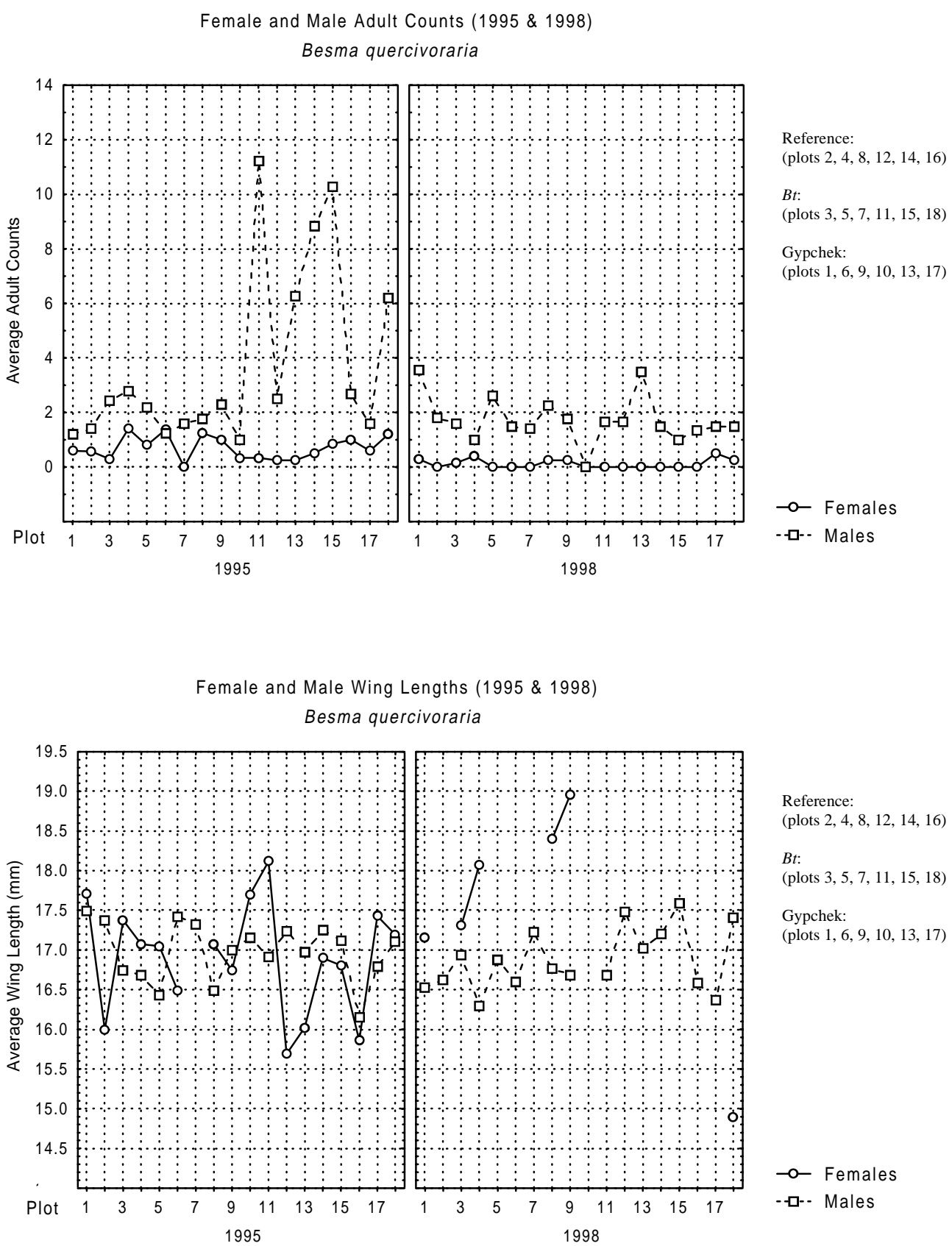

Reference:

(plots 2, 4, 8, 12, 14, 16)

$B t$ :

(plots 3, 5, 7, 11, 15, 18)

Gypchek:

(plots 1, 6, 9, 10, 13, 17)

-o- Females

-ם-- Males

Figure 35. Female and male adult counts and wing lengths (1995 \& 1998). Graphs illustrate average plot counts and wing lengths of male and female Besma quercivoraria collected during the 1995 pretreatment and 1998 treatment years from the George Washington (plots 1-9) and Monongahela (plots 10-18) National Forests. 


\section{Besma endropiaria}

A total of 358 larvae and 2,248 adults was collected during 1995-1998. The graphs in Figure 36 illustrate the average weekly catches. Larvae appear predominantly in the second half of the sampling season, with the exception of a brief peak during week 4 in the GW in 1995. The majority of adults were collected between weeks 2-13.

General trends relative to the plots for larvae and adults are illustrated in the first and second graphs in Figure 37, respectively. The ANOVA for larvae indicated significant main effects for YEAR and FOREST ( $\mathrm{P}<0.001$ and $\mathrm{P}=0.017$, respectively). More larvae were captured during treatment years and more larvae were captured from the MON. The interaction for YEAR x FOREST was significant $(\mathrm{P}=0.012)$, indicating significantly more males captured from the MON during treatment years than any other statistical group. Adults showed a significant difference only for YEAR, with more moths collected during treatment years $(\mathrm{P}=0.002)$.

A total of 441 females and 935 males was identified from1995 and 1998 catches (see the first graph presented in Figure 38). The ANOVA indicated significant differences for SEX, FOREST, and SEX x FOREST ( $\mathrm{P}=0.014, \mathrm{P}=0.005$, and $\mathrm{P}=0.025$, respectively). More males were collected than females and more moths were collected from the MON than from the GW. The interaction indicated that both males and females were significantly more abundant in the MON during 1998 than 1995. Significantly more males were collected during 1998 than females; however, similar numbers of males and females were collected during 1995.

A total of 382 females and 908 males was measured for wing length from the 1995 and 1998 catches. In order to conduct the 4-way ANOVA, averages were supplied for groups with insufficient numbers in 5 instances. The second graph in Figure 38 indicates average wing measurements for both sexes. The resulting ANOVA indicated significant differences for the main effects of YEAR and FOREST $(\mathrm{P}<0.001$ and $\mathrm{P}=0.001$, respectively). Smaller moths were collected during treatment years and smaller moths were collected from the MON (18.626/ 18.235 and $18.670 / 18.190 \mathrm{~mm}$, respectively). Interactions for YEAR x FOREST, SEX x TREATMENT, and YEAR x SEX x TREATMENT were significant $(\mathrm{P}=0.036, \mathrm{P}=0.004$, and $\mathrm{P}=0.040$, respectively). Though interactions were complex, the basic results indicated that females and males were of 
similar size within each forest during each year, with the exception that females in GC plots in the GW were larger than males collected in GC plots from the GW. Wing lengths of moths of both sexes were larger in the GW than in the MON, with the exception that wing lengths of males on treatment plots were of similar size from both forests.

A total of 132 larvae were weighed during 1997 and 1998 treatment years. The 2-way ANOVA (FOREST, TREATMENT) indicated no significant differences. Average larval weights were $.0313 \mathrm{~g}$. Figure 39 illustrates average weights of larvae collected from each plot. 

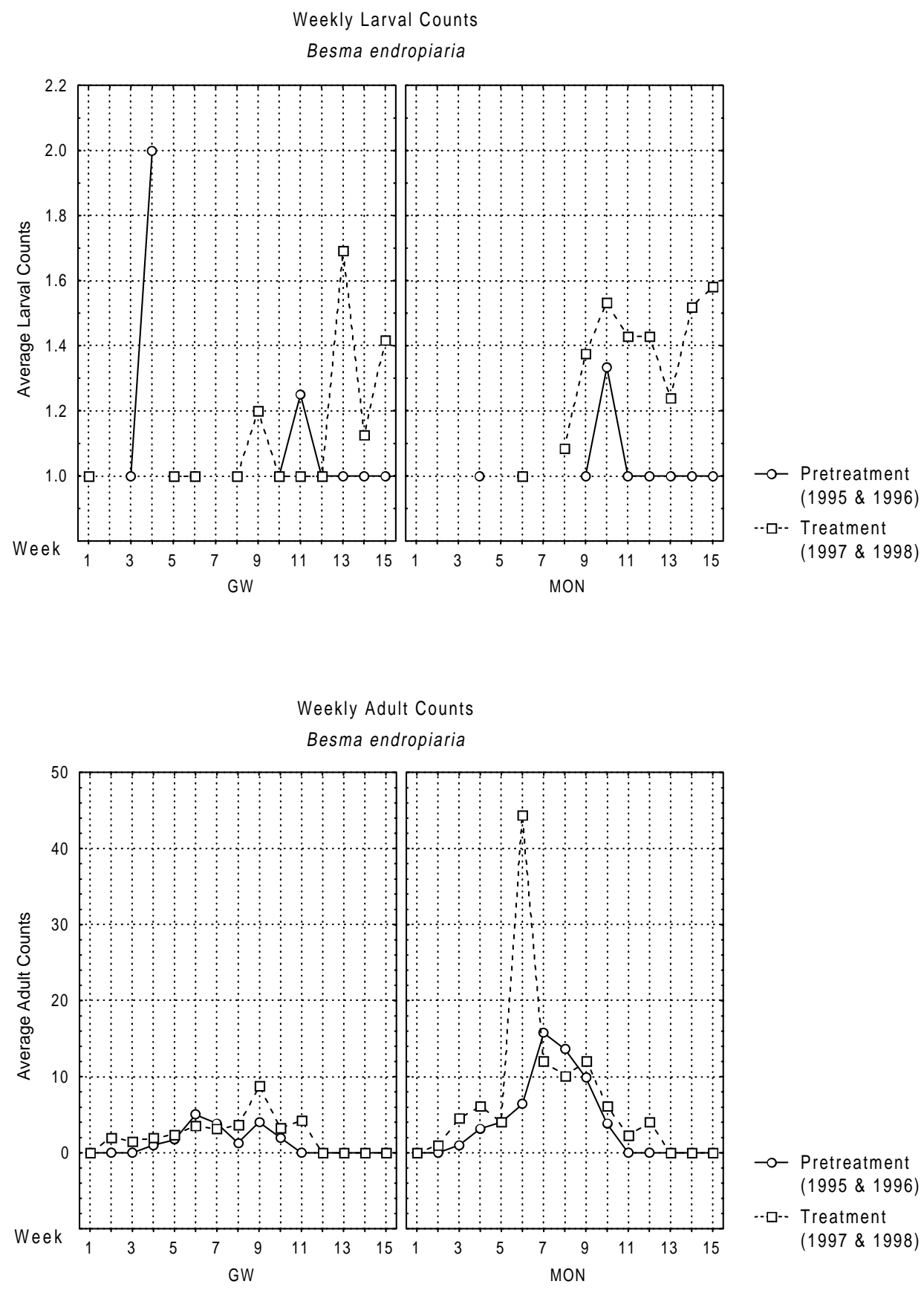

Figure 36. Weekly larval and adult counts (1995-1998). Graphs illustrate average weekly larval and adult counts of Besma endropiaria collected during pretreatment and treatment years from the George Washington (GW) and Monongahela (MON) National Forests. 

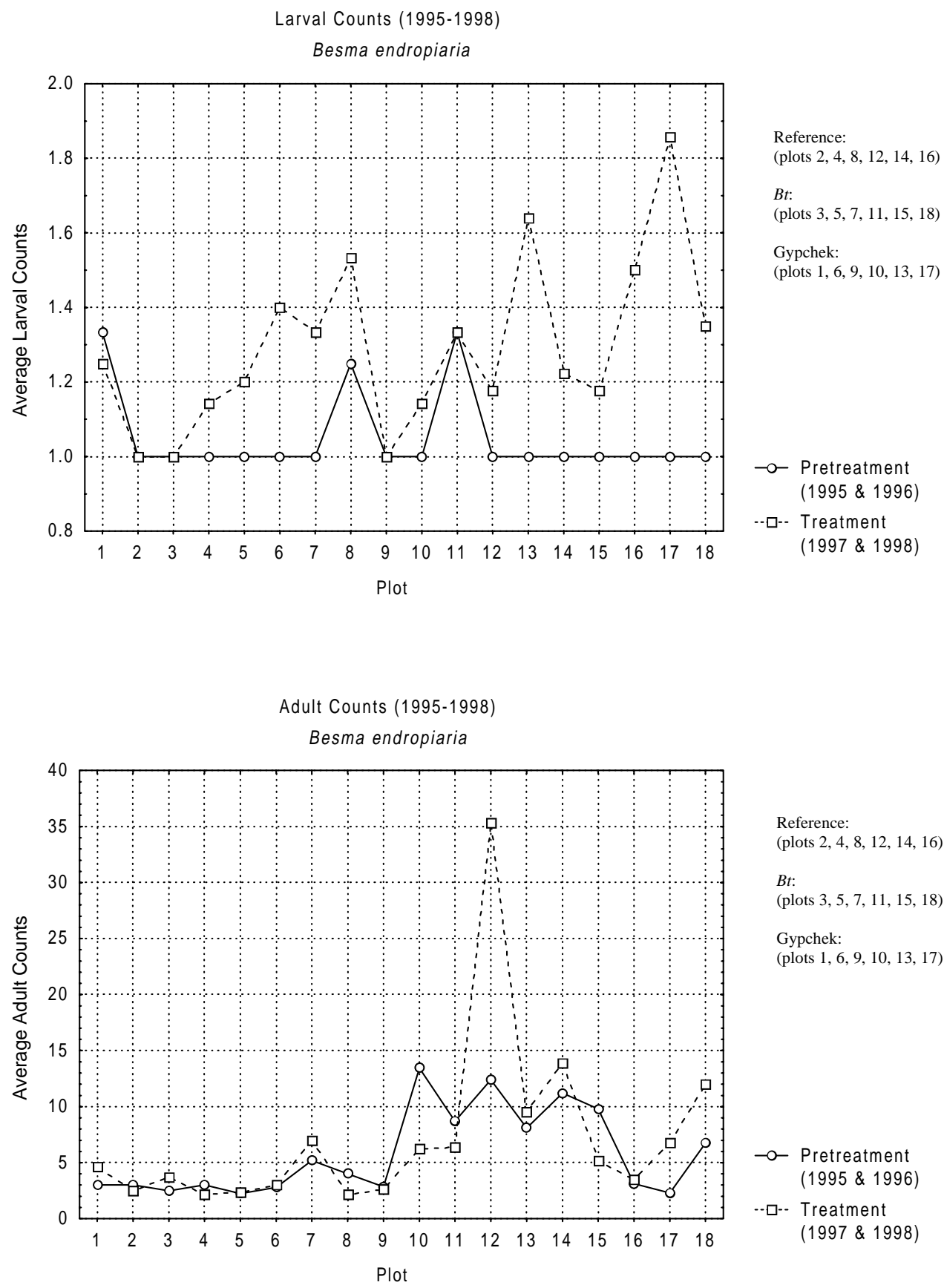

Reference:

(plots 2, 4, 8, 12, 14, 16)

Bt:

(plots $3,5,7,11,15,18$ )

Gypchek:

(plots 1, 6, 9, 10, 13, 17)

- - Pretreatment (1995 \& 1996)

--ם-- Treatment (1997 \& 1998)

Figure 37. Larval and adult counts (1995-1998). Graphs illustrate average plot counts of larval and adult Besma endropiaria collected during pretreatment and treatment years from the George Washington (plots 1-9) and Monongahela (plots 10-18) National Forests. 

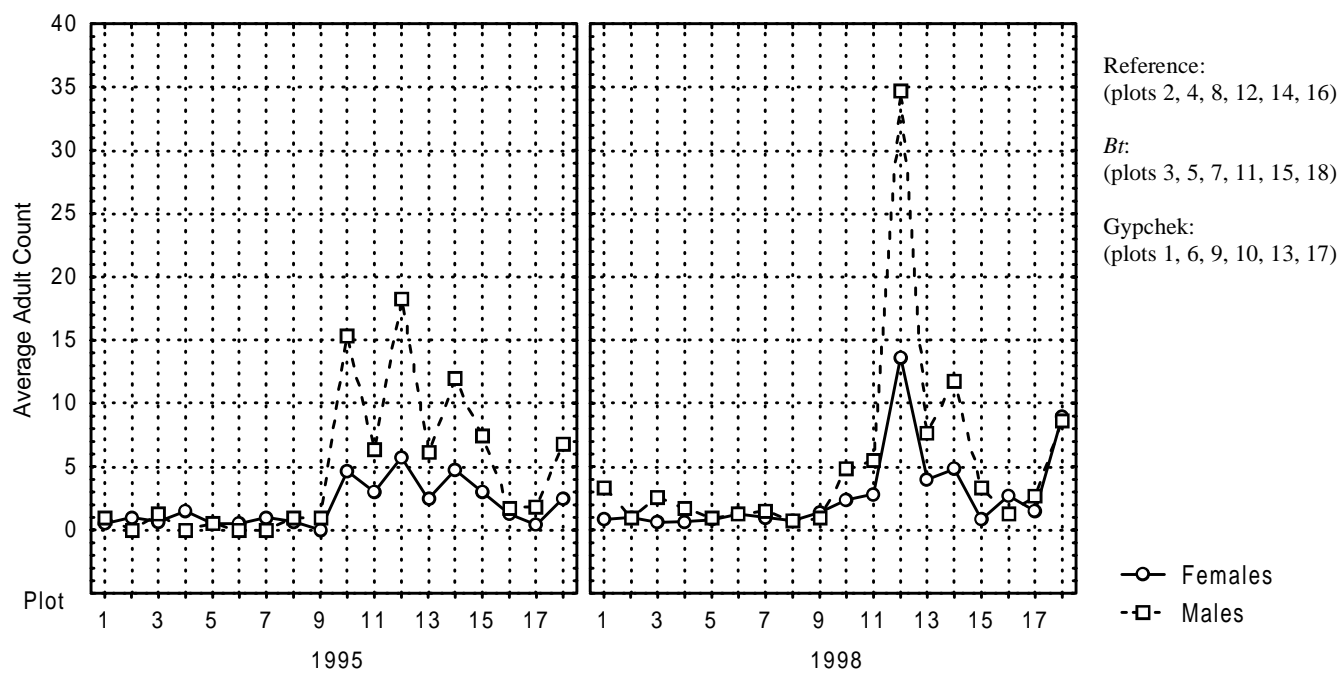

Female and Male Wing Lengths (1995 \& 1998)

Besma endropiaria
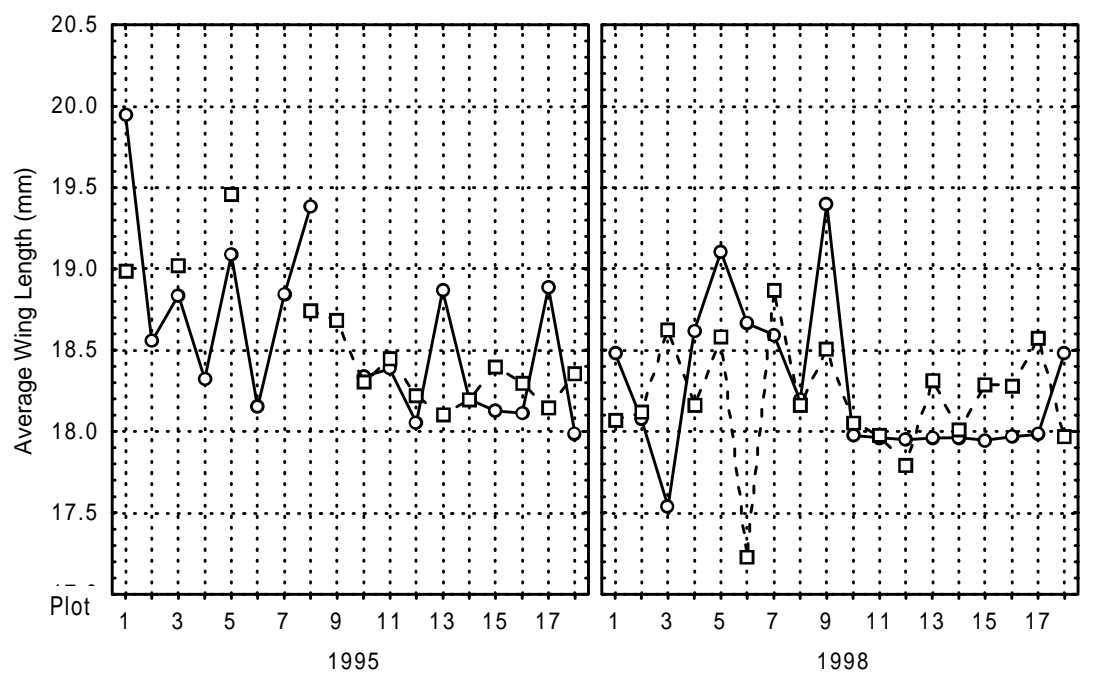

Reference:

(plots 2, 4, 8, 12, 14, 16) $B t$ :

(plots 3, 5, 7, 11, 15, 18)

Gypchek:

(plots 1, 6, 9, 10, 13, 17)

-o- Females

- $\square$ - Males

Figure 38. Female and male adult counts and wing lengths (1995 \& 1998). Graphs illustrate average plot counts and wing lengths for Besma endropiaria collected during the 1995 pretreatment and 1998 treatment years from the George Washington (plots 1-9) and Monongahela (plots 10-18) National Forests. 


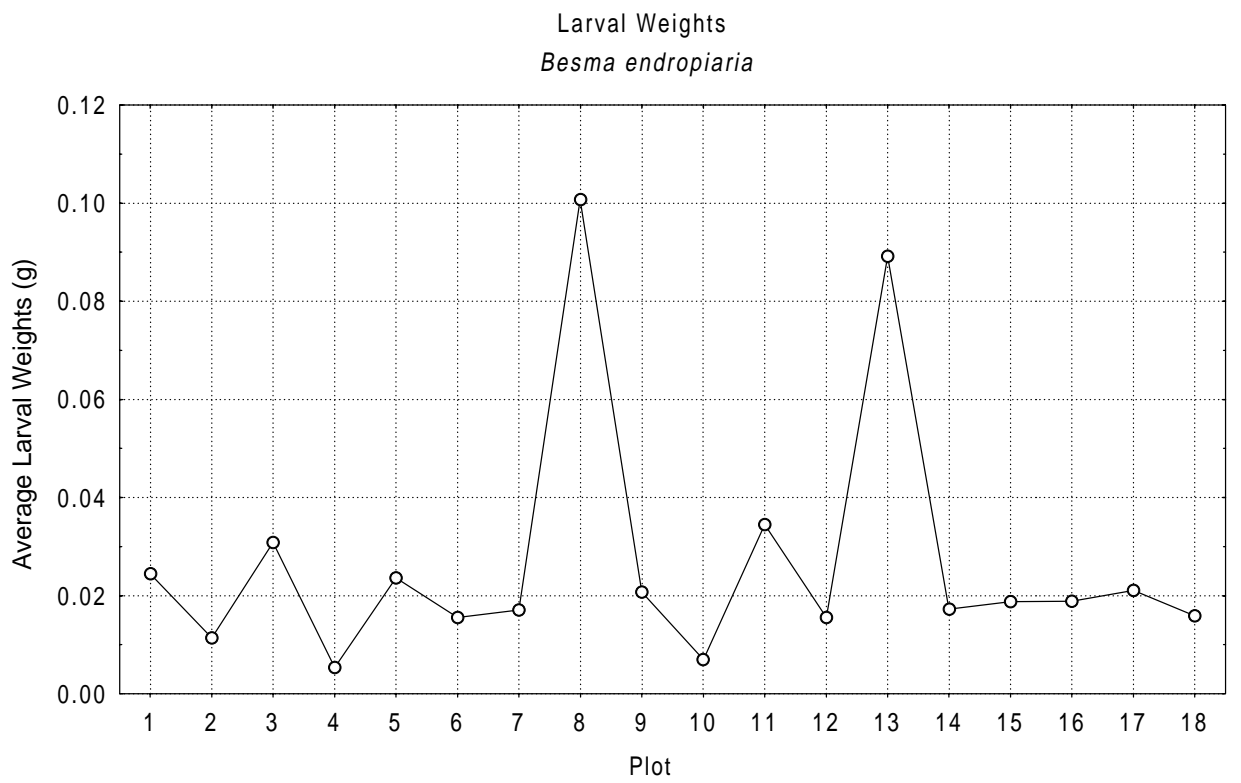

Figure 39. Average larval weights. The graph illustrates the average larval weights of Besma endropiaria collected from each plot during the 1997 and 1998 treatment years from the George Washington (plots 19) and Monongahela (plots 10-18) National Forests. 


\section{Malacosoma disstria}

A total of 39 larvae and 1,460 adults was collected during 1995-1998. The graphs in Figure 40 illustrate the average weekly catches. All larvae were collected during the first 7 weeks of the sampling seasons. Adults appeared between weeks 4-12 in the GW and between weeks 6-15 in the MON.

General trends for adults collected from each plot are illustrated in the first graph in Figure 41. An ANOVA indicated a significant interaction for YEAR $x$ TREATMENT $(\mathrm{P}=0.034)$. The interaction is

illustrated as the second graph of Figure 41. Though significantly fewer individuals were collected from $B t$ treated plots during treatment years, they were not significantly fewer than any of the statistical groups collected during pretreatment years.

A total of 22 female and 829 male moths was identified from 1995 and 1998 catches (see first graph in Figure 42). Main effects of SEX and FOREST were significant ( $\mathrm{P}=0.001$ and $\mathrm{P}=0.027$, respectively). More males were captured than females, while in general, more moths were collected from the GW than from the MON. The interactions of SEX x YEAR and SEX x FOREST were significant $(\mathrm{P}=0.025$ and $\mathrm{P}=0.039$, respectively). More males were captured from the GW than any other statistical group. More individuals were captured during 1998 from the GW than any other statistical group.

A total of 22 female and 772 male adults was measured for wing length from 1995 and 1998 catches (see the second graph presented in Figure 42). Females were larger than males with an average wing length of $19.979 \mathrm{~mm}$. Male wing length averaged $14.287 \mathrm{~mm}$. Due to insufficient numbers an ANOVA was not conducted on these individuals. 

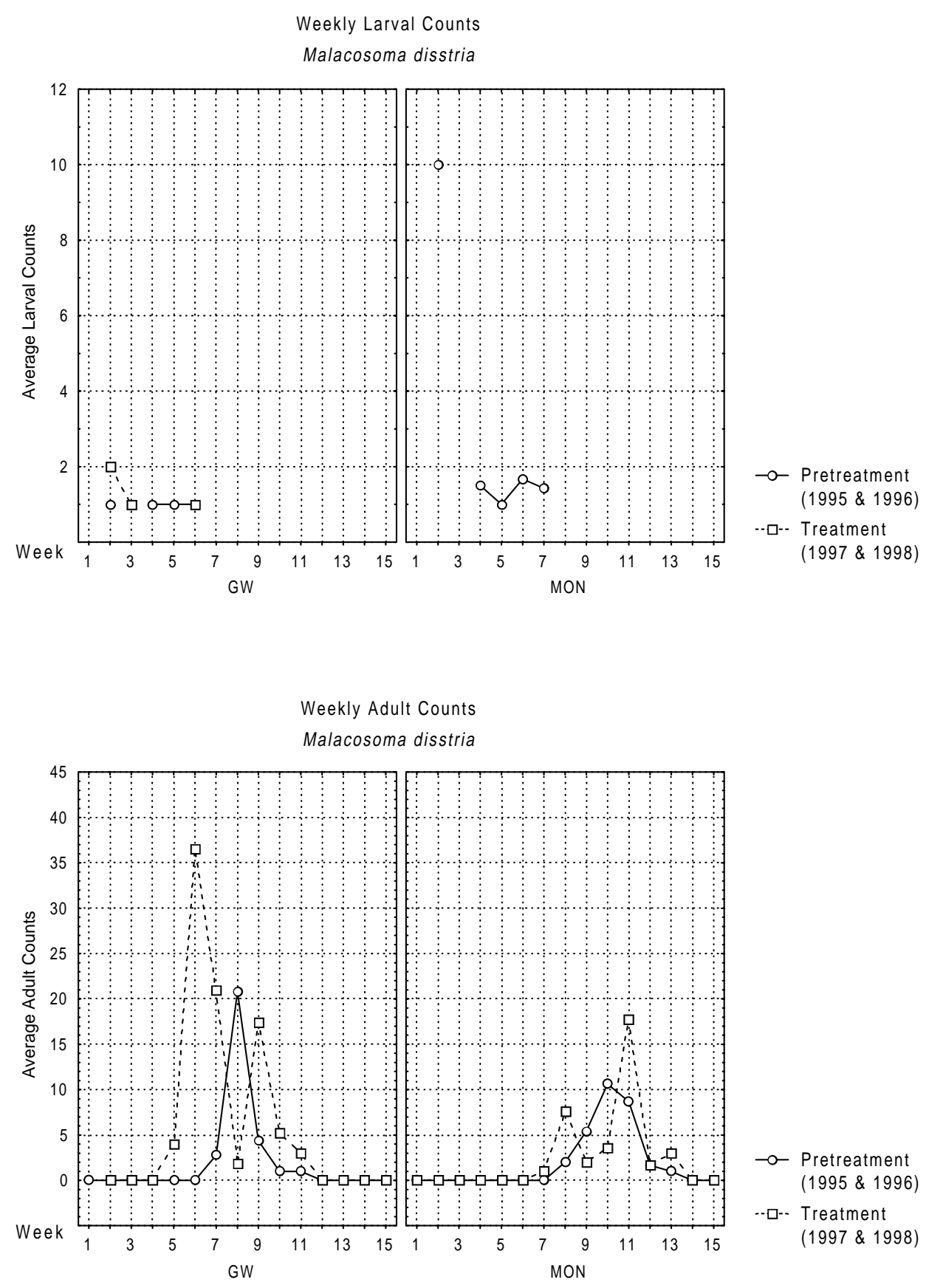

Figure 40. Weekly larval and adult counts (1995-1998). Graphs illustrate average weekly larval and adult counts of Malacosoma disstria collected during pretreatment and treatment years from the George Washington (GW) and Monongahela (MON) National Forests. 

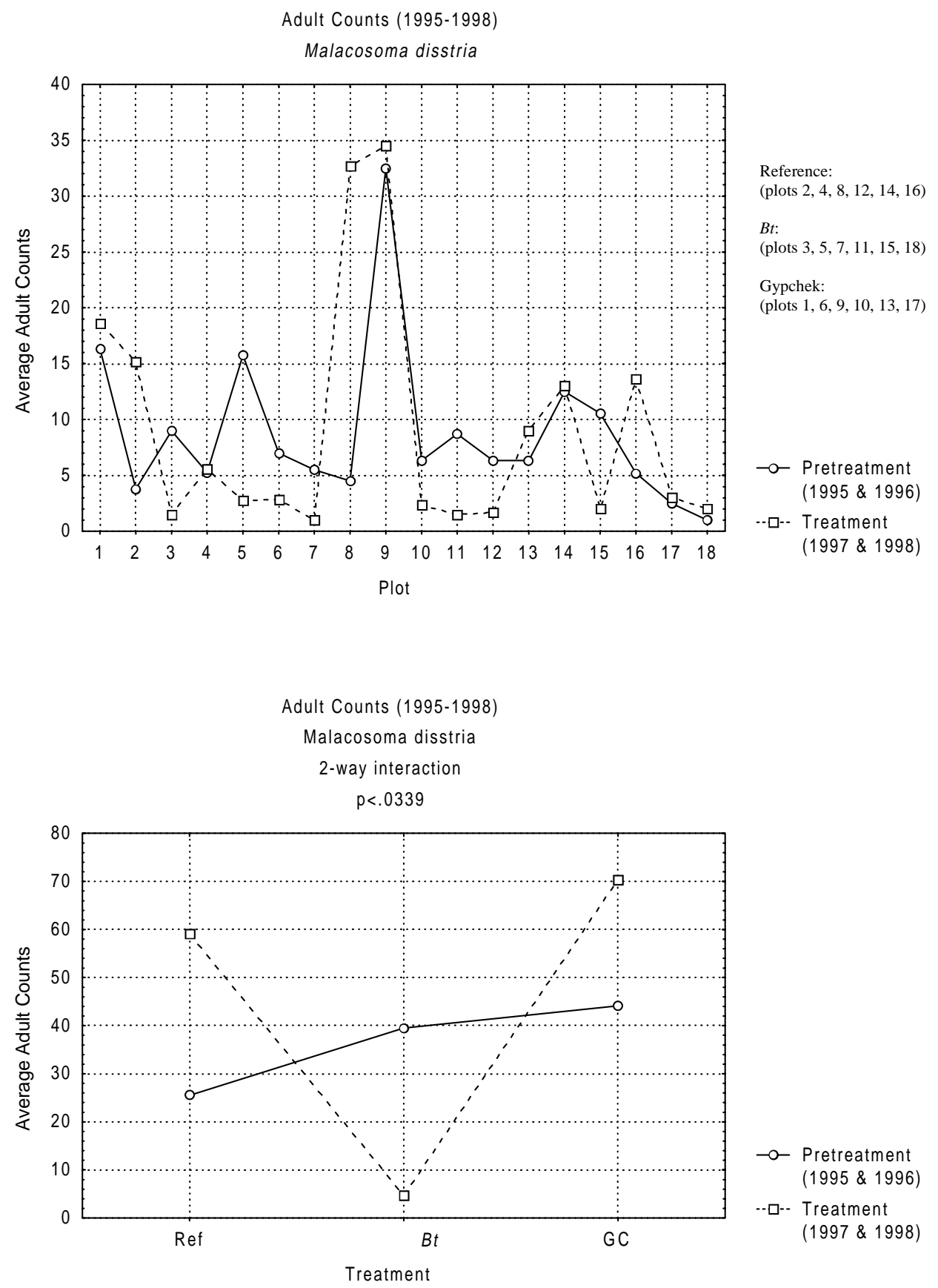

Figure 41. Adult counts and interaction (1995-1998). The first graph illustrates average plot counts of Malacosoma disstria adults collected during pretreatment and treatment years from the George Washington (plots 1-9) and Monongahela (plots 10-18) National Forests. The second graph illustrates count interaction between treatment categories and pretreatment/treatment years. 
Female and Male Adult Counts

Malacosoma disstria
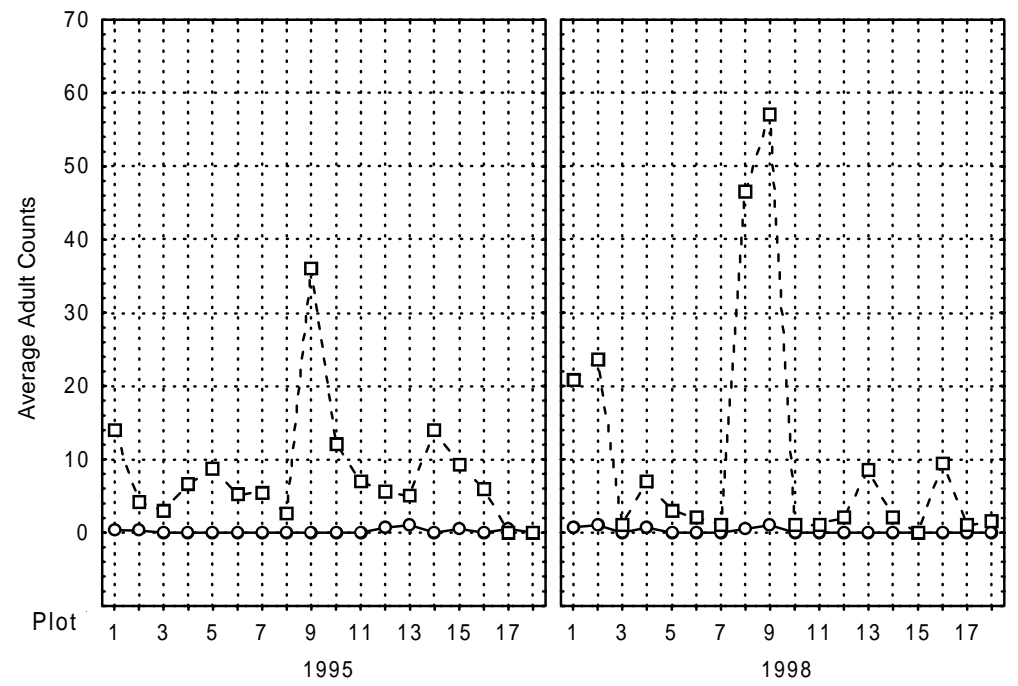

Female and Male Wing Lengths

Malacosoma disstria
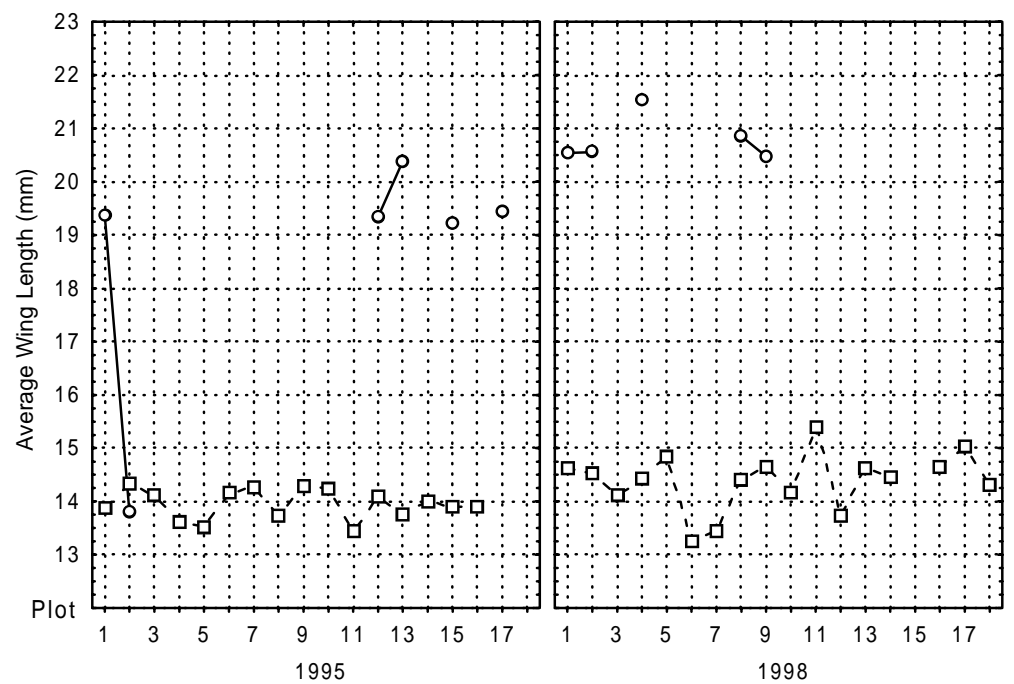

Reference:

(plots 2, 4, 8, 12, 14, 16)

$B t$ :

(plots 3, 5, 7, 11, 15, 18)

Gypchek:

(plots 1, 6, 9, 10, 13, 17)

-o- Females

-- - - Males

Reference:

(plots 2, 4, 8, 12, 14, 16)

$B t$ :

(plots 3, 5, 7, 11, 15, 18)

Gypchek:

(plots 1, 6, 9, 10, 13, 17)

$\rightarrow$ Females

-- - Males

Figure 42. Female and male adult counts and wing lengths (1995 \& 1998). Graphs illustrate average plot counts and wing lengths for male and female Malacosoma disstria collected during the 1995 pretreatment and 1998 treatment years from the George Washington (plots 1-9) and Monongahela (plots 10-18) National Forests. 


\section{Heterocampa guttivitta}

A total of 298 larvae and 4,312 adults was collected during 1995-1998. The graphs in Figure 43 illustrate the average weekly catches. Larvae were collected primarily during the second halves of the sampling seasons. Adults were collected primarily between weeks 2-12.

General trends relative to the plots are presented for larvae and adults as the first and second graphs in Figure 44, respectively. For larvae, the ANOVA indicated significant main effects for YEAR and FOREST ( $\mathrm{P}=0.021$ and $\mathrm{P}=0.023$, respectively). More larvae were collected during treatment years and more larvae were collected from the MON. Interactions for YEAR x FOREST and YEAR x TREATMENT were also significant $(\mathrm{P}=0.003$ and $\mathrm{P}=0.039$, respectively). More larvae were collected from the MON during 1998 than any other statistical group. More larvae were collected from Ref plots during 1998 than from Ref and GC plots of the pretreatment years. Furthermore, more larvae were collected from 1998 Ref plots than from 1998 Bt plots. This interaction is presented as the third graph in Figure 44.

The significant ANOVA effects for the adults were YEAR, FOREST, and YEAR x FOREST $(\mathrm{P}=0.001, \mathrm{P}=0.048$, and $\mathrm{P}=0.022$, respectively). The larger number of moths in catches from 1998 compared to 1995 is attributed to those collected during 1998 from the MON.

A total of 58 female and 2, 391 male moths was identified from 1995 and 1998 catches (see first graph in Figure 45). The main effects for SEX and YEAR were significant $(\mathrm{P}<0.001$ and $\mathrm{P}=0.001$, respectively). More males were collected than females and more moths were collected during 1998 than during 1995. The interaction SEX x YEAR $(\mathrm{P}=0.002)$ attributes the increase in 1998 to an increase in the number of males collected during that year, though males were significantly more plentiful in catches than females both years.

Females were larger on average than males with an average wing length of $22.445 \mathrm{~mm}$ compared to 20.641 mm (see second graph in Figure 45). Females, however, were not included in the ANOVA due to insufficient numbers. A total of 2,441 males were measured and the ANOVA indicated significant effects 
for FOREST and YEAR x FOREST $(\mathrm{P}<0.001$ and $\mathrm{P}=0.005$, respectively). Larger males were collected

from the MON than from the GW. Males collected during 1998 from the GW were smaller than 1995 males collected from the GW. Males collected from the MON were of similar size both years. 

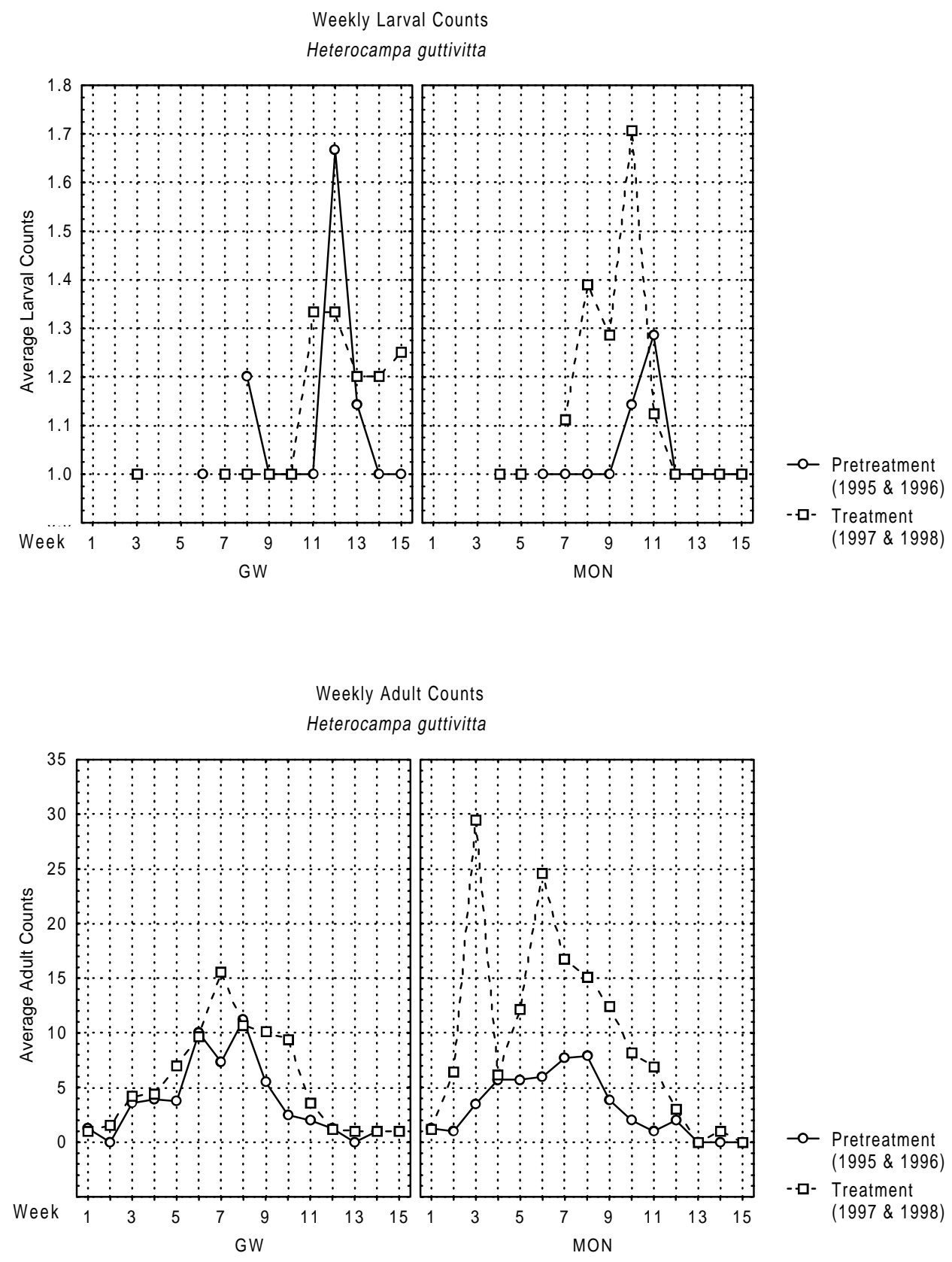

Figure 43. Weekly larval and adult counts (1995-1998). Graphs illustrate average weekly larval and adult counts of Heterocampa guttivitta collected during pretreatment and treatment years from the George Washington (GW) and Monongahela (MON) National Forests. 


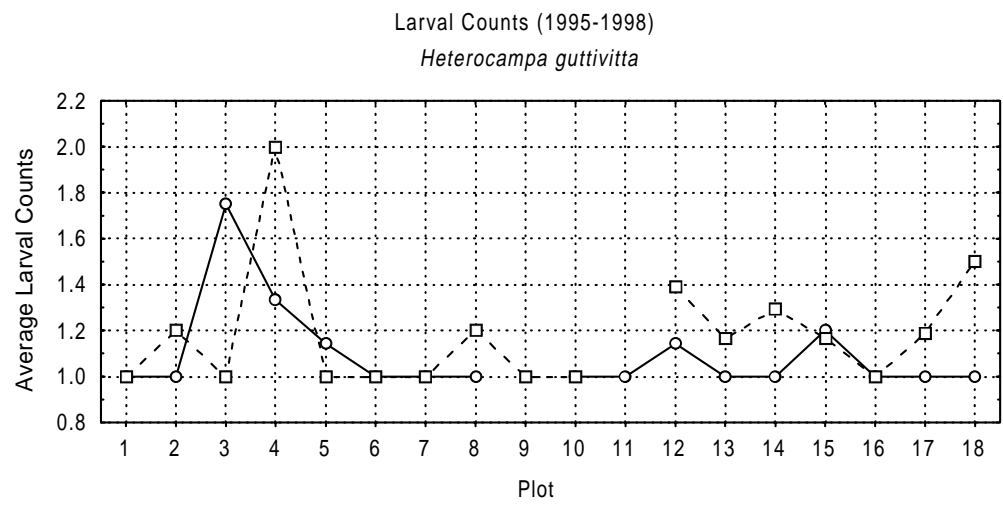

Reference:

(plots 2, 4, 8, 12, 14, 16)

$B t$ :

(plots 3, 5, 7, 11, 15, 18)

Gypchek:

(plots 1, 6, 9, 10, 13, 17)

Adult Counts (1995-1998)

Heterocamp guttivitta

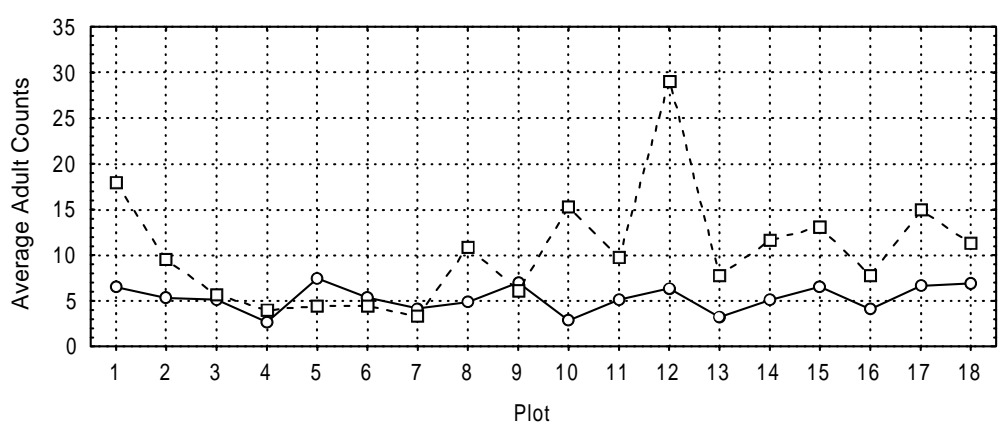

Reference:

(plots 2, 4, 8, 12, 14, 16)

$B t$ :

(plots 3, 5, 7, 11, 15, 18)

Gypchek:

(plots 1, 6, 9, 10, 13, 17)

-o- Pretreatment

(1995 \& 1996)

- $\square$ - Treatment

(1997 \& 1998)

Larval Counts (1995-1998)

Heterocampa guttivitta

2-way interaction

$p<.0390$

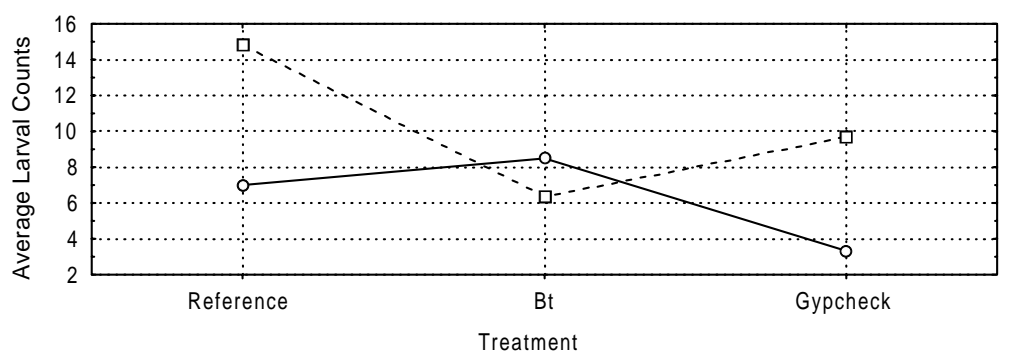

-o- Pretreatment (1995 \& 1996)

- $\square$ - Treatment

(1997 \& 1998)

Figure 44. Larval and adult counts with larval interaction (1995-1998). The first two graphs illustrate average plot counts for larval and adult Heterocampa guttivitta collected during pretreatment and treatment years from the George Washington (plots 1-9) and Monongahela (plots 10-18) National Forests. The third graph illustrates larval count interaction between treatment categories and pretreatment/treatment years. 

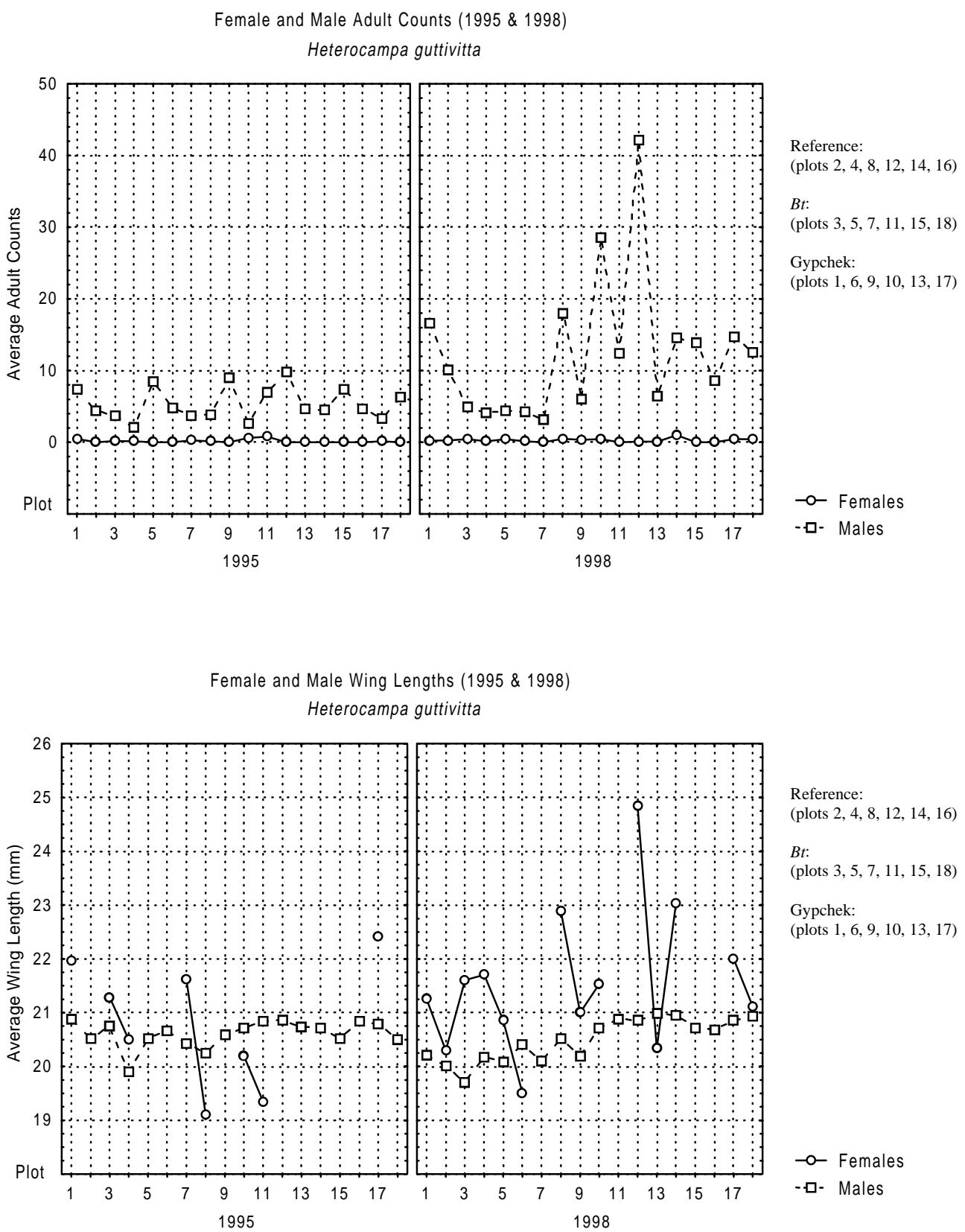

Figure 45. Female and male adult counts and wing lengths (1995 \& 1998). Graphs illustrate average plot counts and wing lengths of Heterocampa guttivitta collected during the 1995 pretreatment and 1998 treatment years from the George Washington (plots 1-9) and Monongahela (plots 10-18) National Forests. 


\section{Nadata gibbosa}

A total of 183 larvae and 3,134 adults was collected during 1995-1998. The graphs in Figure 46 illustrate average weekly catches. The majority of larvae appear after week 8 in the GW and after week 11 in the MON. A peak was not observed in the population collected from the MON during treatment years. Adults showed a general peak in abundance in the GW toward the middle of the sampling season. Adults collected from the MON appeared slightly delayed by comparison.

General trends relative to the plots for larvae and adults are presented as the first and second graphs of Figure 47, respectively. The ANOVA results indicated that less larvae and adults were collected during treatment years $(\mathrm{P}<0.001$ and $\mathrm{P}=0.024$, respectively).

A total of 91 female and 1,614 male moths was identified from 1995 and 1998 catches (see first graph in Figure 48). Males were significantly more abundant in light trap catches than females $(\mathrm{P}<0.001)$. Significantly less moths were collected during treatment years $(\mathrm{P}<0.001)$ than during pretreatment years. The SEX x YEAR interaction $(\mathrm{P}<0.001)$ indicated that less females were captured than males each year; however significantly fewer males were collected during 1998 compared with 1995.

The wing length of females was larger on average than males $(27.351 \mathrm{~mm}$ and $24.095 \mathrm{~mm}$, respectively). The trend in size is illustrated in the second graph in Figure 48. All females were measured; however, insufficient numbers were collected for inclusion in the ANOVA. A total of 1,630 males were measured for wing length. The ANOVA conducted on the males indicated no significant differences. 

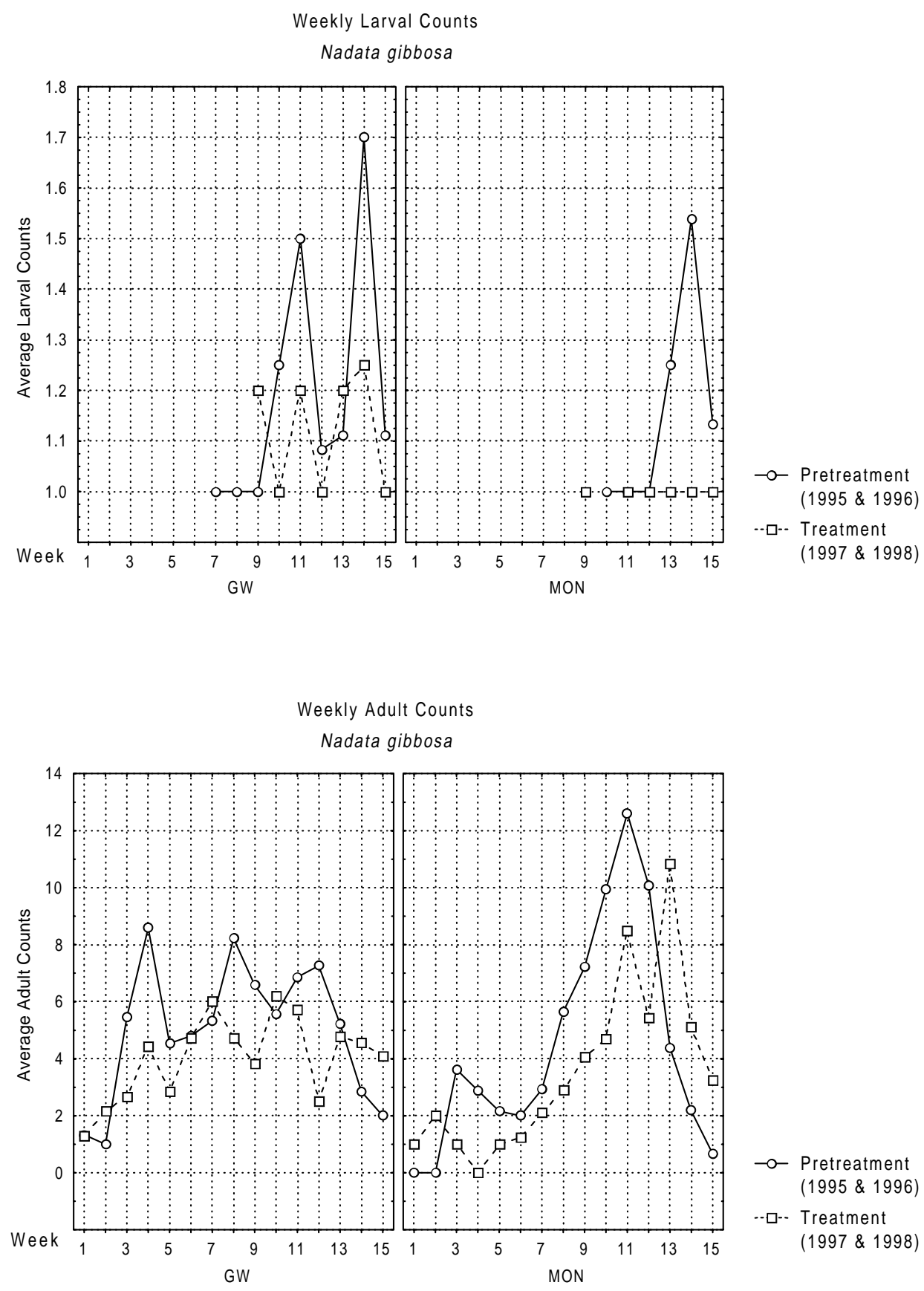

Figure 46. Weekly larval and adult counts (1995-1998). Graphs illustrate average weekly larval and adult counts of Nadata gibbosa collected during pretreatment and treatment years from the George Washington (GW) and Monongahela (MON) National Forests. 


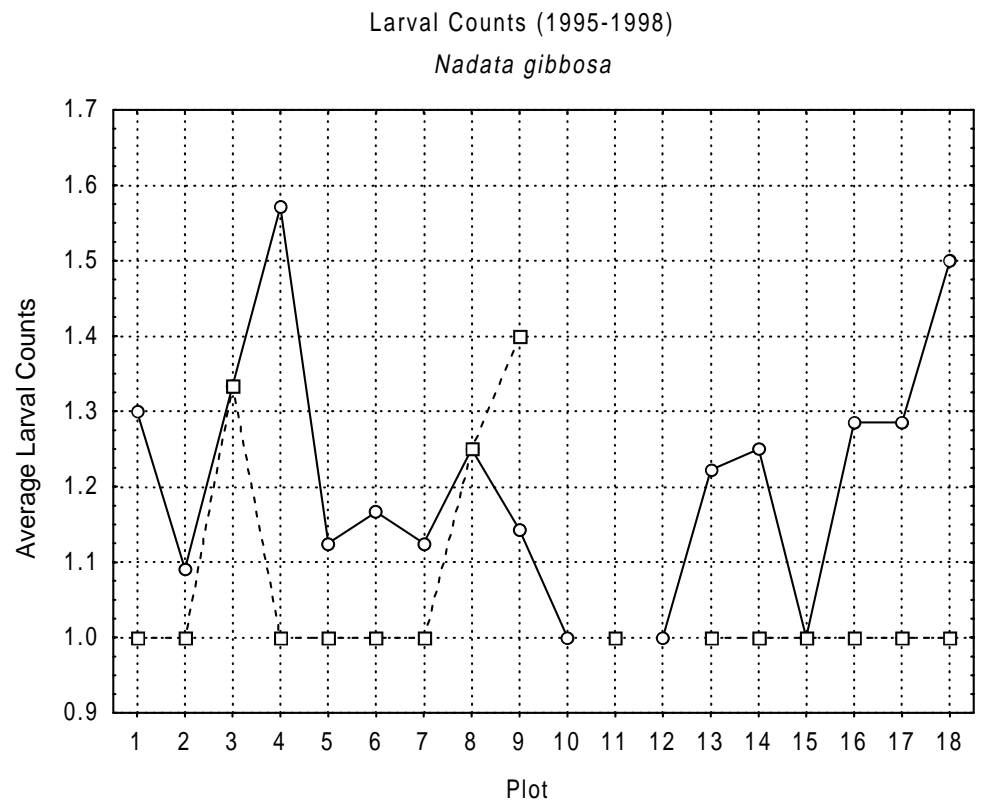

Reference:

(plots 2, 4, 8, 12, 14, 16)

Bt:

(plots 3, 5, 7, 11, 15, 18)

Gypchek:

(plots 1, 6, 9, 10, 13, 17)

- - Pretreatment

(1995 \& 1996)

- $\square$ - Treatment

(1997 \& 1998)

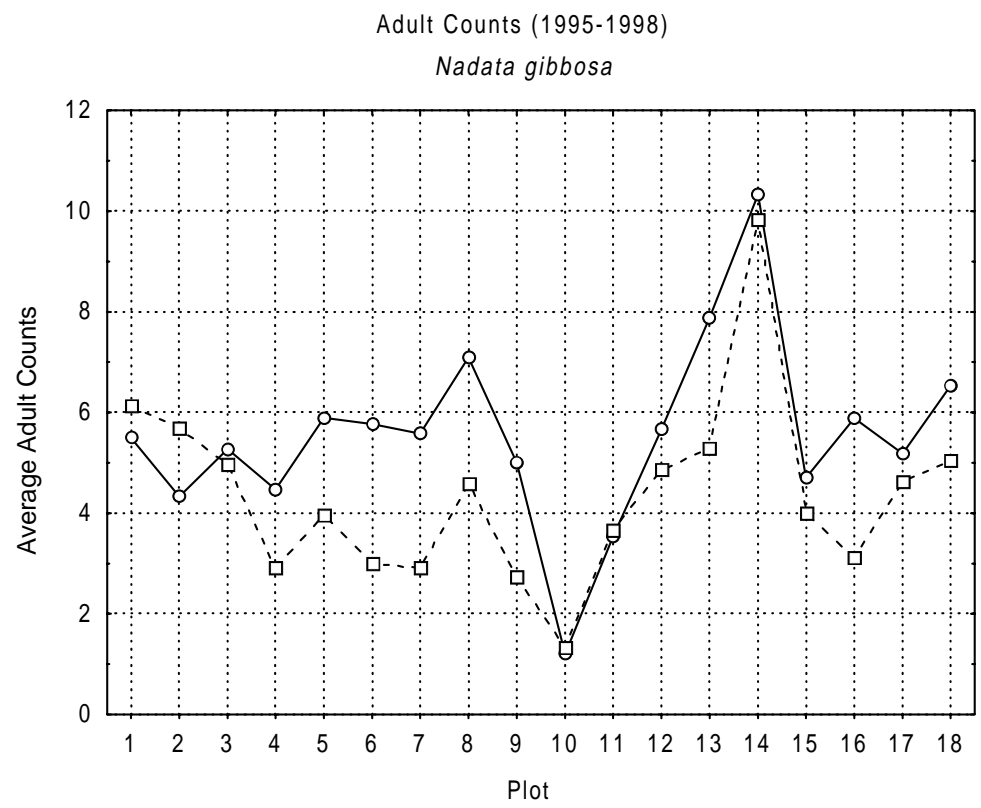

Reference:

(plots 2, 4, 8, 12, 14, 16)

$B t$ :

(plots $3,5,7,11,15,18$ )

Gypchek:

(plots 1, 6, 9, 10, 13, 17)

- - Pretreatment

(1995 \& 1996)

--ロ-- Treatment

(1997 \& 1998)

Figure 47. Larval and adult counts (1995-1998). Graphs illustrate average plot counts of larval and adult Nadata gibbosa collected during pretreatment and treatment years from the George Washington (plots 19) and Monongahela (plots 10-18) National Forests. 
Female and Male Adult Counts (1995 \& 1998)

Nadata gibbosa
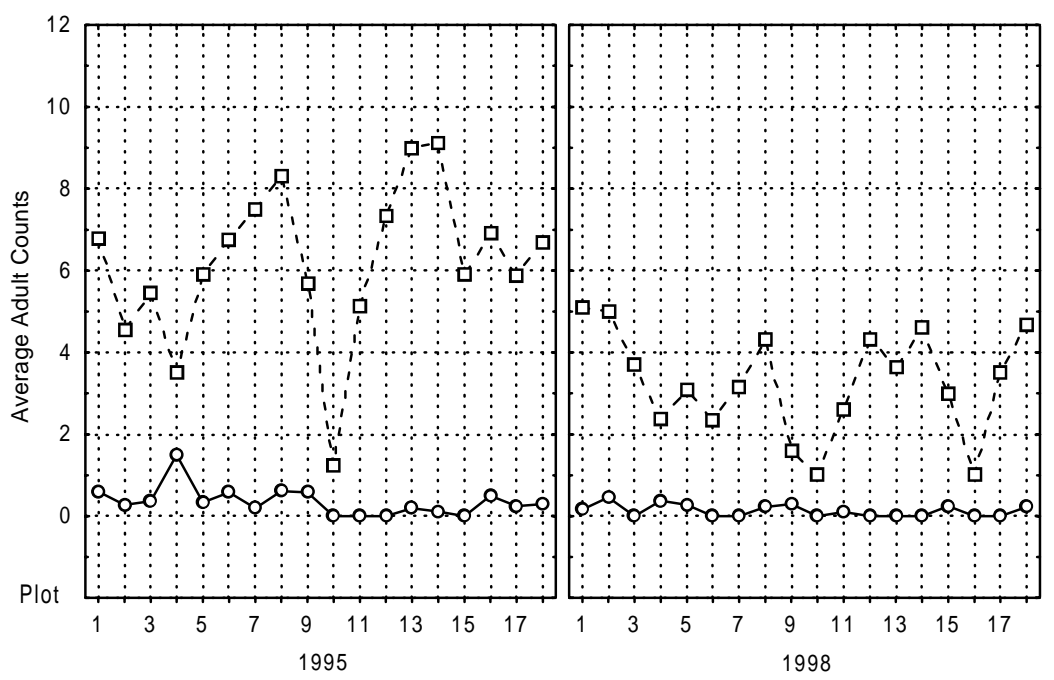

Female and Male Wing Lengths (1995 \& 1998) Nadata gibbosa

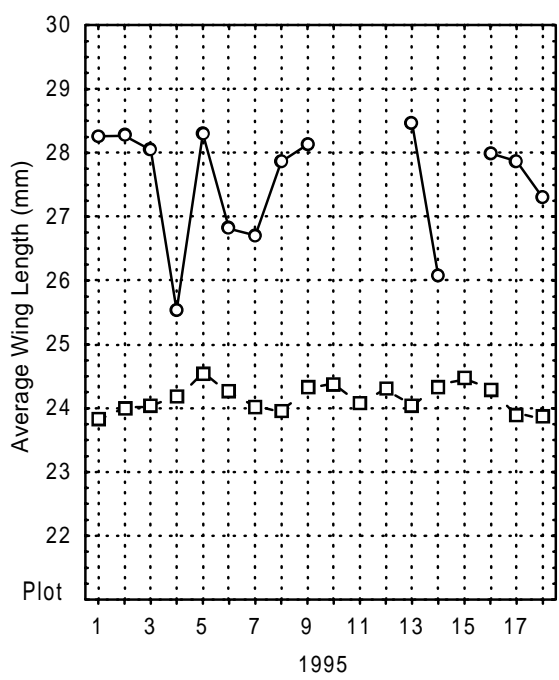

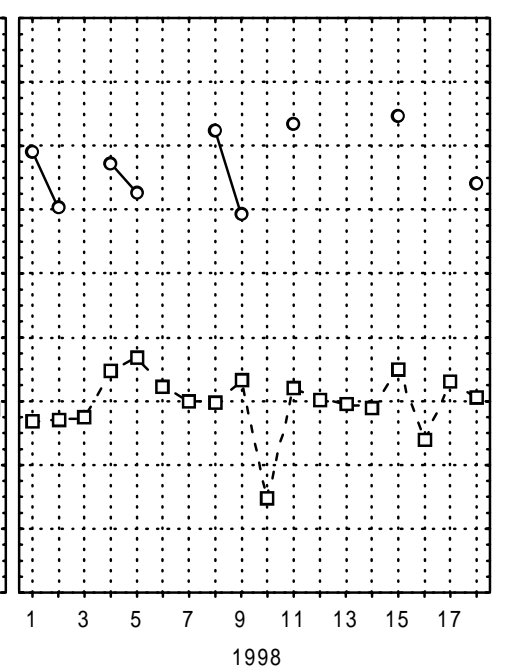

Reference:

(plots 2, 4, 8, 12, 14, 16)

Bt:

(plots $3,5,7,11,15,18$ )

Gypchek:

(plots $1,6,9,10,13,17$ )

-o- Females

$-\square$ - Males

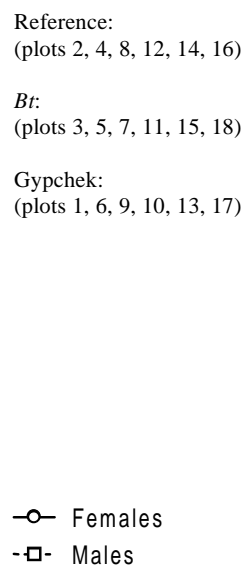

Figure 48. Female and male adult counts and wing lengths (1995 \& 1998). Graphs illustrate average plot counts and wing lengths of female and male Nadata gibbosa collected during the 1995 pretreatment and 1998 treatment years from the George Washington (plots1-9) and Monongahela (plots 10-18) National Forests. 


\section{Hypoprepia fucosa}

A total of 347 larvae and 12,540 adults was collected during 1995-1998. The graphs in Figure 49 illustrate the average weekly catches. The bulk of larvae were collected from the GW through the first nine weeks of the sampling seasons. The majority of the MON larvae were collected between weeks 2-12. A peak in larval counts was not apparent in the MON for treatment years. Adults in the GW were most abundant in catches between weeks 7-15. The MON moths showed peak abundance in catches beginning approximately 2 weeks later than in the GW.

General plot trends for larvae and adults are presented in the first and second graphs of Figure 50, respectively. The ANOVA indicated a significant decline in larvae and adults collected during treatment years $(\mathrm{P}=0.001$ and 0.003 , respectively).

A total of 456 female and 6,274 male moths was identified from 1995 and 1998 catches. Adult counts and size differences are illustrated in the first and second graphs in Figure 51, respectively. Males were significantly more abundant in catches $(\mathrm{P}<0.001)$ and significantly fewer moths were collected in 1998 than in $1995(\mathrm{P}<0.001)$. A significant interaction for SEX x YEAR was also identified $(\mathrm{P}<0.001)$. Though males were more abundant during both years, significantly fewer were collected during 1998 than during 1995. Female abundance remained approximately the same.

A total of 450 females and 4,114 males were measured for wing length from 1995 and 1998 catches. Females were significantly larger than males $(\mathrm{P}<0.001)$. An interaction for SEX $x$ YEAR $x$ FOREST was also identified ( $\mathrm{P}=0.016)$. Although females were larger than males both years in both forests, females were significantly smaller in the MON than in the GW during 1998. The $1998 \mathrm{MON}$ females were also smaller than the 1995 MON females. 
Weekly Larval Counts

Hypoprepia fucosa
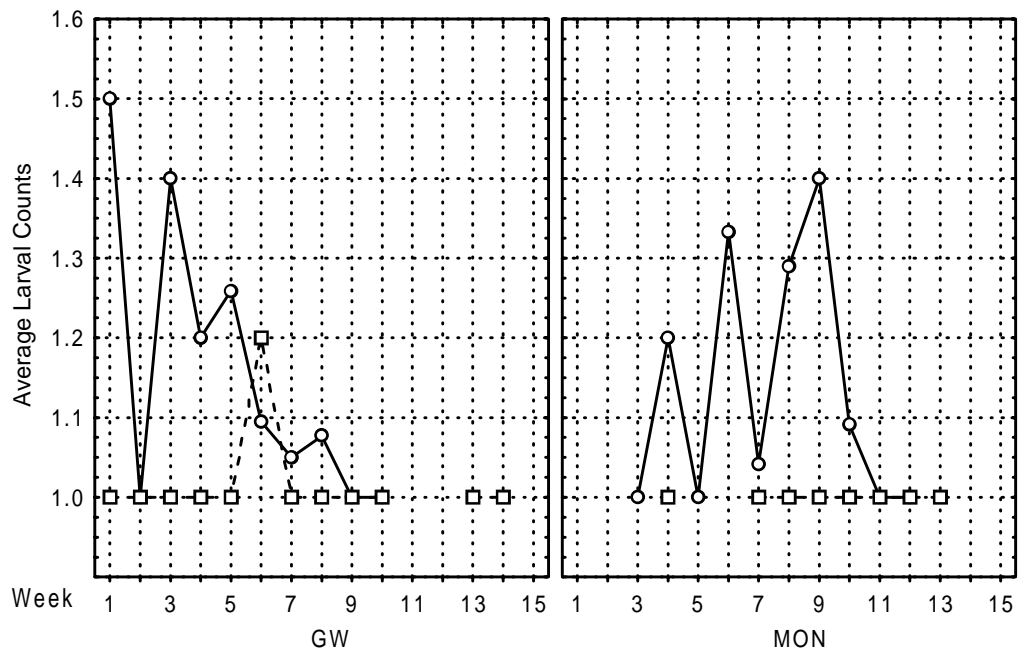

-o- Pretreatment

(1995 \& 1996)

- $\square$ - Treatment

(1997 \& 1998)

Weekly Adult Counts

Hypoprepia fucosa
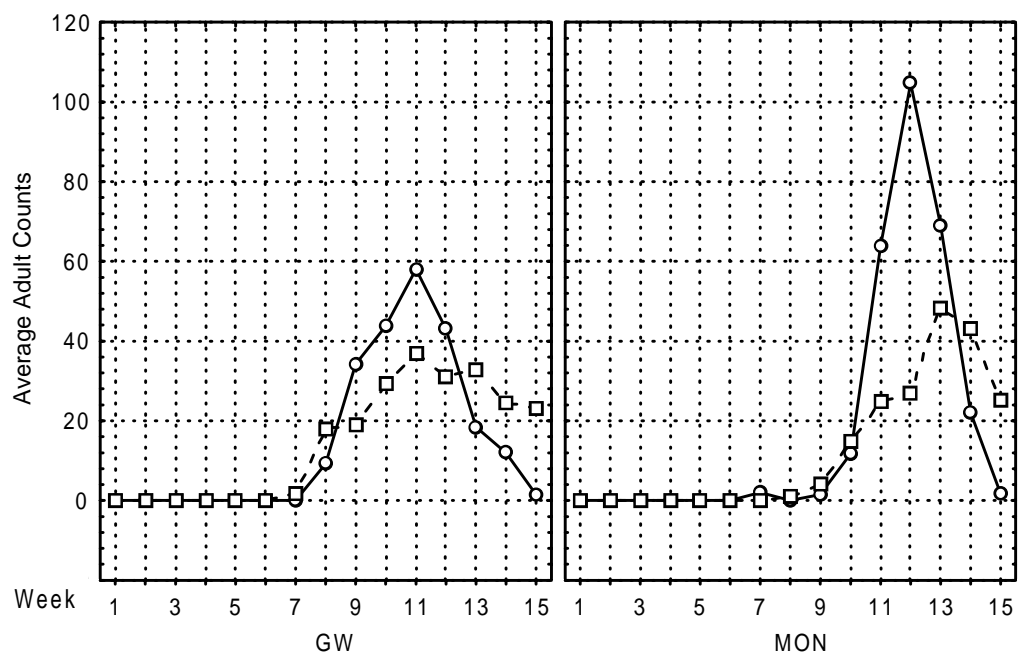

-o- Pretreatment (1995 \& 1996)

- $\square$ - Treatment

(1997 \& 1998)

Figure 49. Weekly larval and adult counts (1995-1998). Graphs illustrate average weekly larval and adult counts of Hypoprepia fucosa collected from during pretreatment and treatment years from the George Washington (GW) and Monongahela (MON) National Forests. 


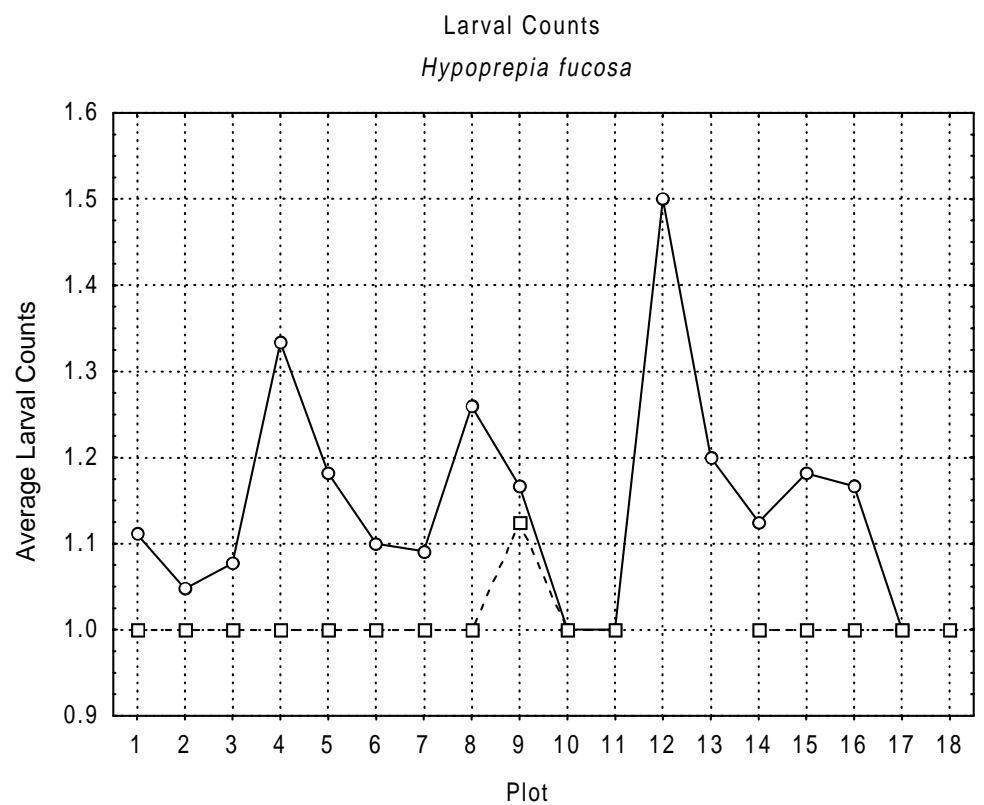

Reference:

(plots 2, 4, 8, 12, 14, 16)

Bt:

(plots $3,5,7,11,15,18)$

Gypchek:

(plots 1, 6, 9, 10, 13, 17)

- - Pretreatment

(1995 \& 1996)

--口-- Treatment

(1997 \& 1998)

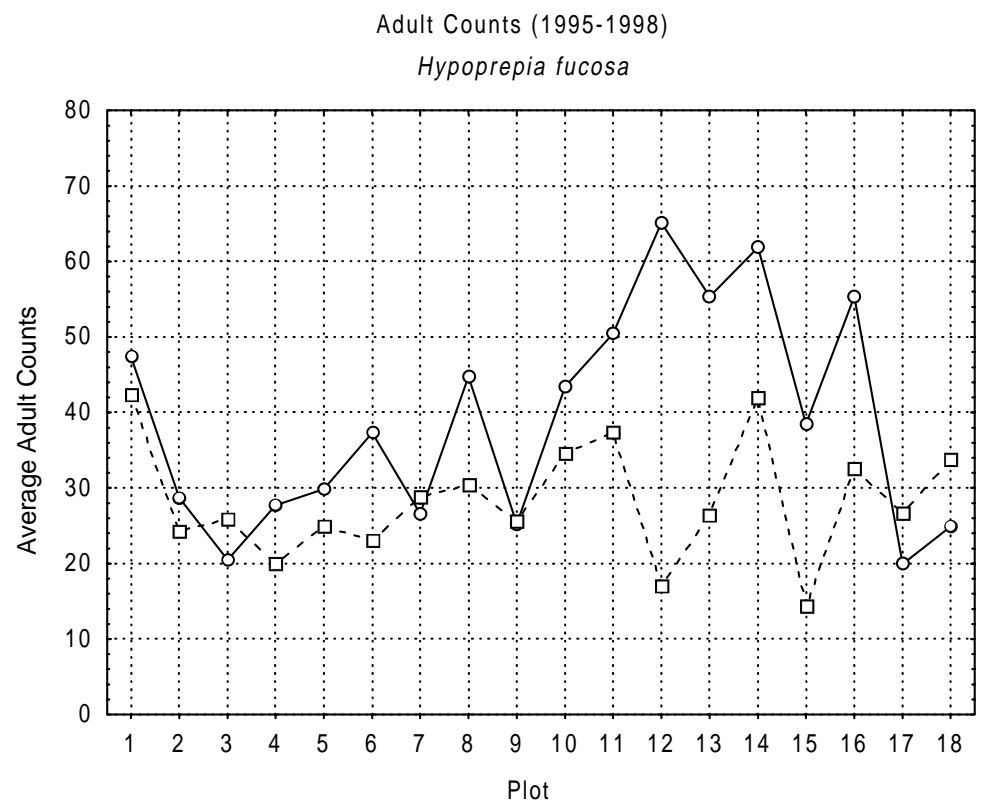

Reference:

(plots 2, 4, 8, 12, 14, 16)

$B t$ :

(plots $3,5,7,11,15,18)$

Gypchek:

(plots 1, 6, 9, 10, 13, 17)

- Pretreatment

(1995 \& 1996)

--ロ-- Treatment

(1997 \& 1998)

Figure 50. Larval and adult counts (1995-1998). Graphs illustrate average plot counts of larval and adult Hypoprepia fucosa collected during pretreatment and treatment years from the George Washington (plots 1-9) and Monongahela (plots 10-18) National Forests. 

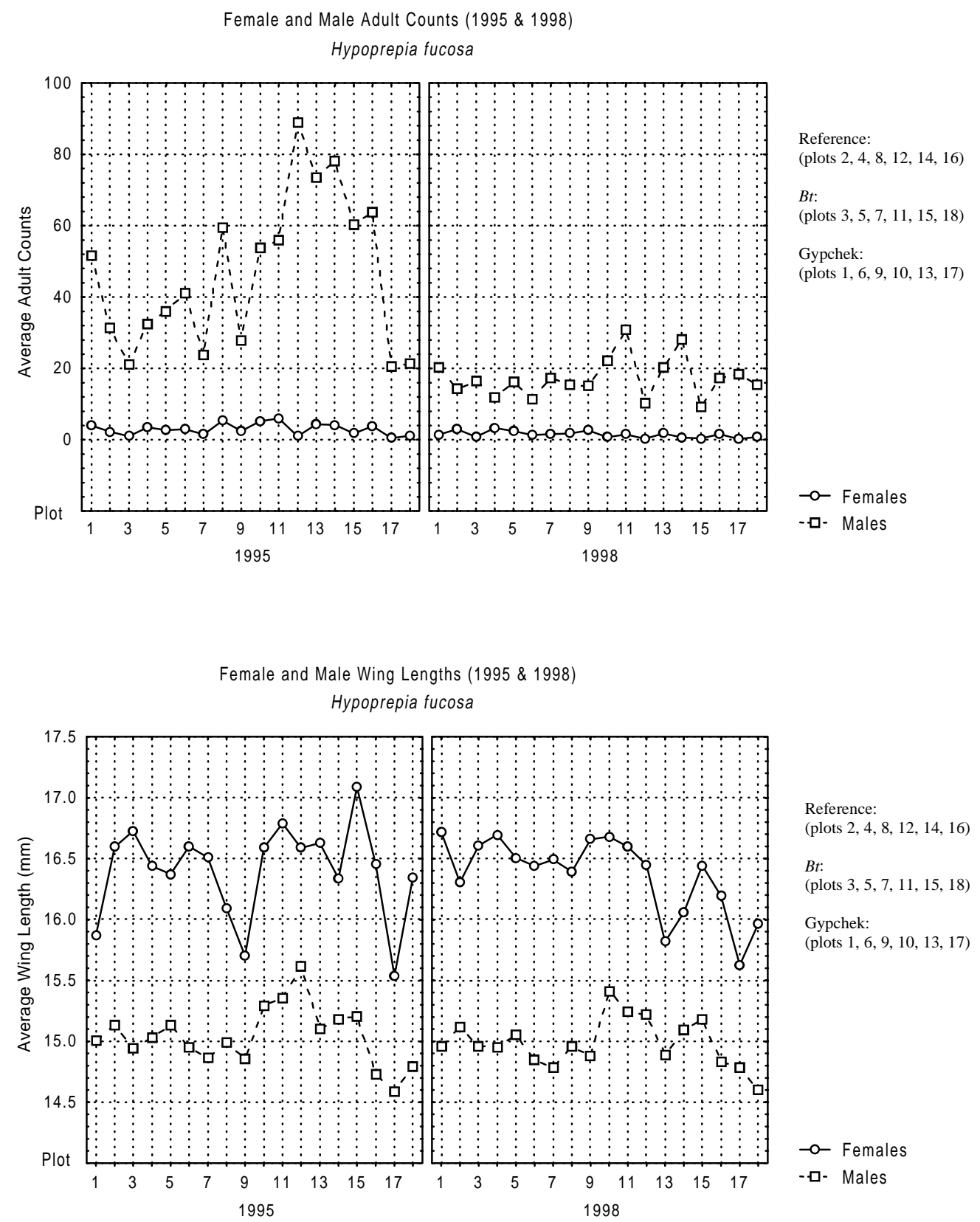

Figure 51. Female and male adult counts and wing lengths (1995 \& 1998). Graphs illustrate average plot counts and wing lengths of female and male Hypoprepia fucosa collected during the 1995 pretreatment and 1998 treatment years from the George Washington (plots 1-9) and Monongahela (plots 10-18) National Forests. 


\section{Halysidota tesselaris}

A total of 144 larvae and 7, 477 adults was collected during 1995-1998. The graphs in Figure 52 illustrate the average weekly catches. Larvae appeared primarily during the last 4 weeks of the sampling season. The majority of adults appeared between weeks 5-15.

General plot trends for larvae and adults are presented in the first and second graphs in Figure 53, respectively. The ANOVA for larvae showed significant effects for YEAR, YEAR x FOREST, and YEAR x TREATMENT $(\mathrm{P}<0.001, \mathrm{P}=0.043$, and $\mathrm{P}=0.017$, respectively). Significantly fewer larvae were collected during treatment years. The YEAR x FOREST interaction indicated that more larvae were collected from the GW during 1995 than any other group. The YEAR x TREATMENT effect showed significantly more larvae collected during 1995 from GC plots than any of the 1998 treatment groups. During 1995, larvae collected from GC plots were also more abundant than those collected from Ref plots during the same year. The interaction is illustrated as the third graph in Figure 53.

Adults showed significant effects for YEAR and for the YEAR x FOREST interaction ( $\mathrm{P}=0.004$ and $\mathrm{P}=0.008$, respectively). More moths were collected during treatment years and the interaction indicated that significantly more moths were collected from the MON during this period than during pretreatment years.

A total of 163 female and 2,311 male adults were identified from 1995 and 1998 catches (see first graph in Figure 54). The difference in male and female counts was significant $(\mathrm{P}<0.001)$. Of these moths, 153 females and 2,224 males were measured for wing length. For the analysis of variance, group averages were substituted in two instances due to insufficient numbers of moths per category. The second graph in Figure 54 illustrates the trend in wing length measurements. Significant differences in wing length for SEX, FOREST, and SEX x YEAR, were identified ( $\mathrm{p}=000, \mathrm{P}=0.046$, and $\mathrm{P}=0.008$, respectively). Females were larger than males and the moths collected from the GW were larger than those collected from the MON. The interaction indicated that though females were larger than males both years, females collected during 
1995 were larger than those collected from 1998. Males showed no significant differences in size between years. 

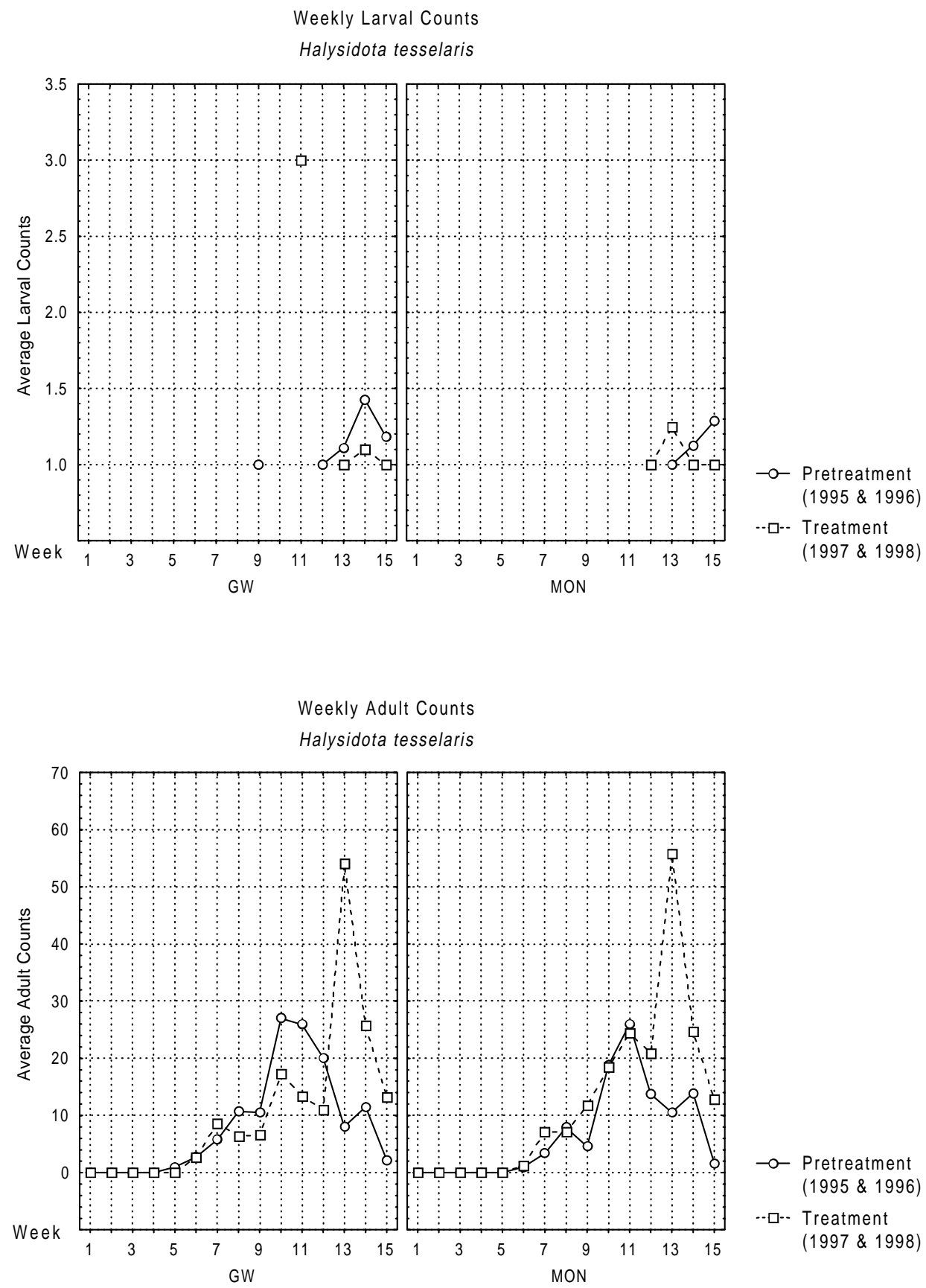

Figure 52. Weekly larval and adult counts (1995-1998). Graphs illustrate average weekly larval and adult counts of Halysidota tesselaris collected during pretreatment and treatment years from the George Washington (GW) and Monongahela (MON) National Forests. 

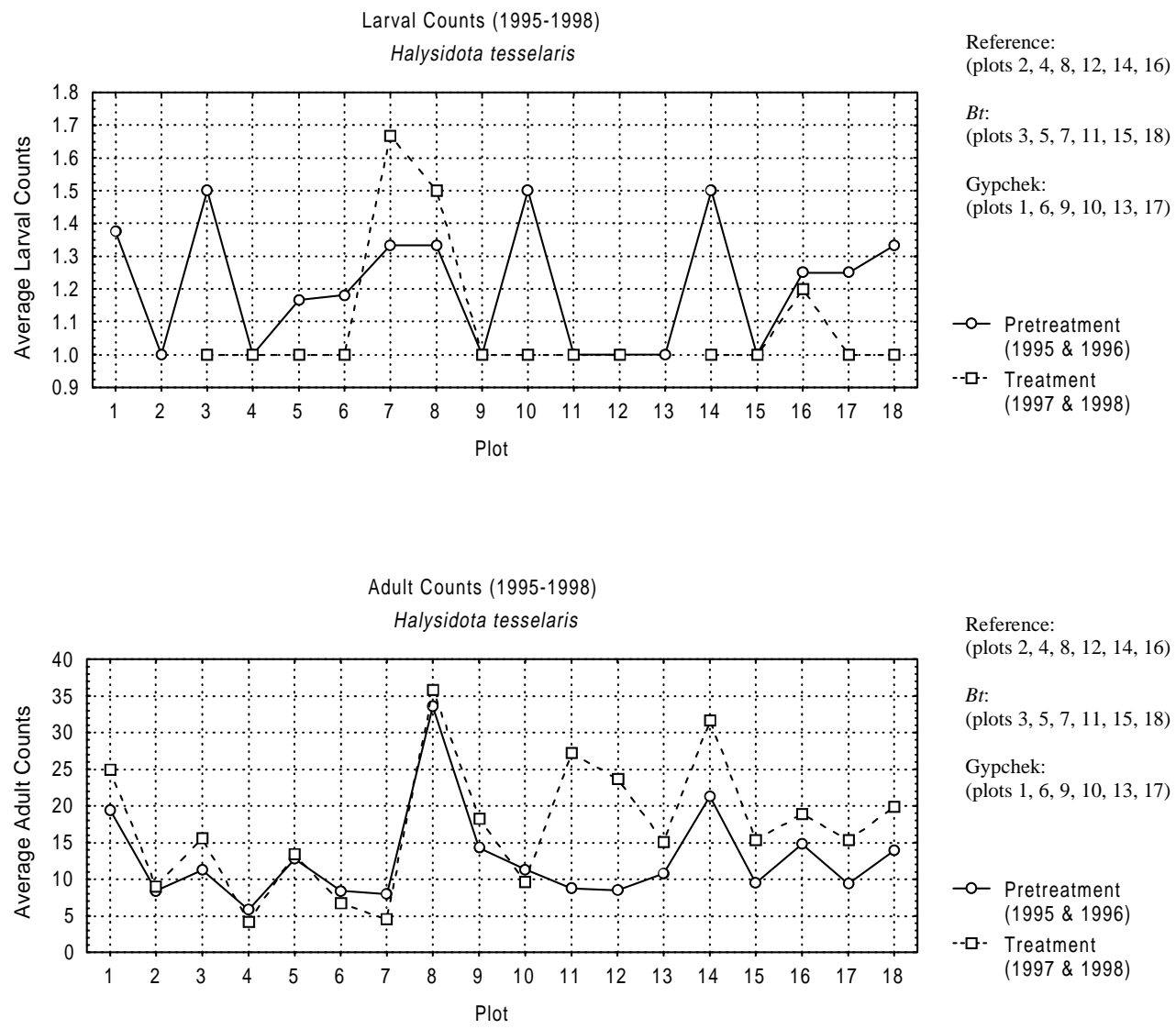

Reference:

(plots 2, 4, 8, 12, 14, 16)

Bt:

(plots 3, 5, 7, 11, 15, 18)

Gypchek

(plots 1, 6, 9, 10, 13, 17)

-o- Pretreatment

(1995 \& 1996)

- $\square$ - Treatment

(1997 \& 1998)

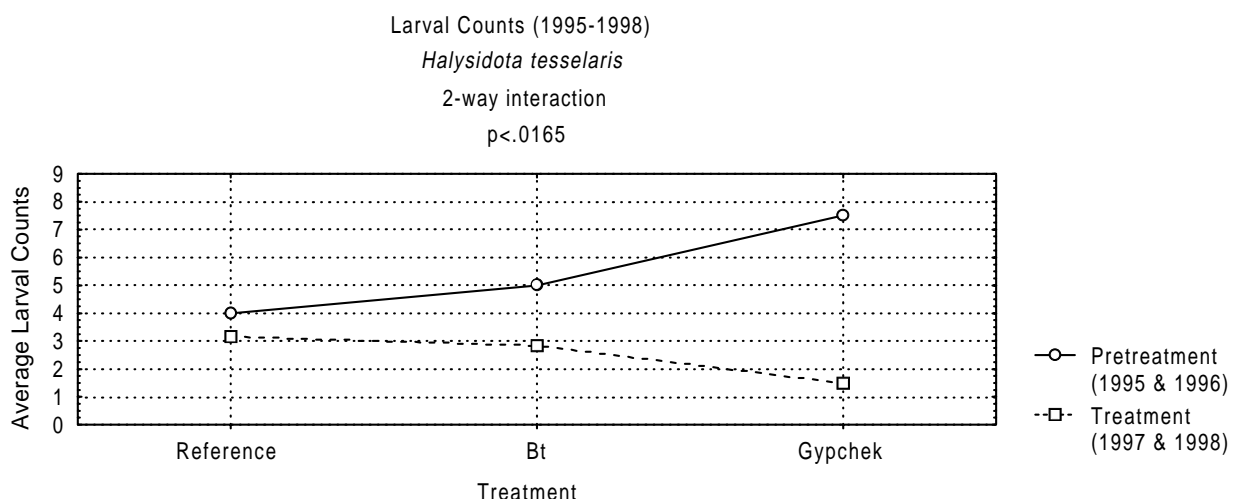

Figure 53. Larval and adult counts and larval interaction (1995-1998). The first two graphs illustrate the average plot counts of larval and adult Halysidota tesselaris collected during treatment years from the George Washington (plots 1-9) and Monongahela (plots 10-18) National Forests. The third graph illustrates larval count interaction of treatment categories with pretreatment/treatment years. 
Female and Male Counts (1995 \& 1998)

Halysidota tessalaris
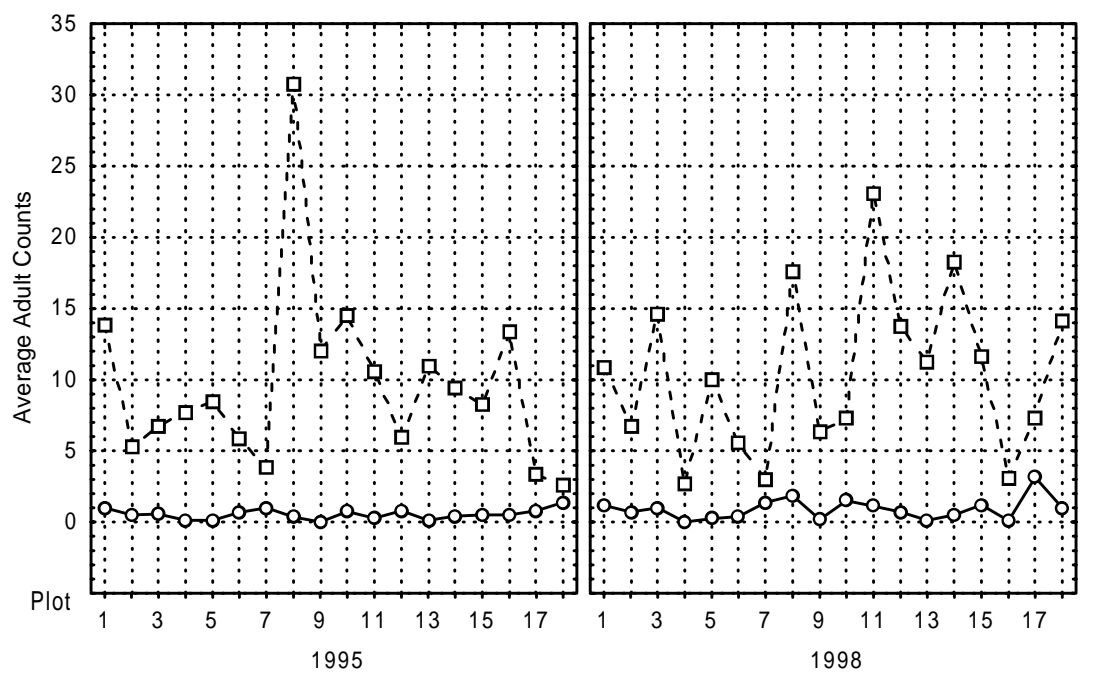

Female and Male Wing Lengths (1995 \& 1998)

Halysidota tesselaris

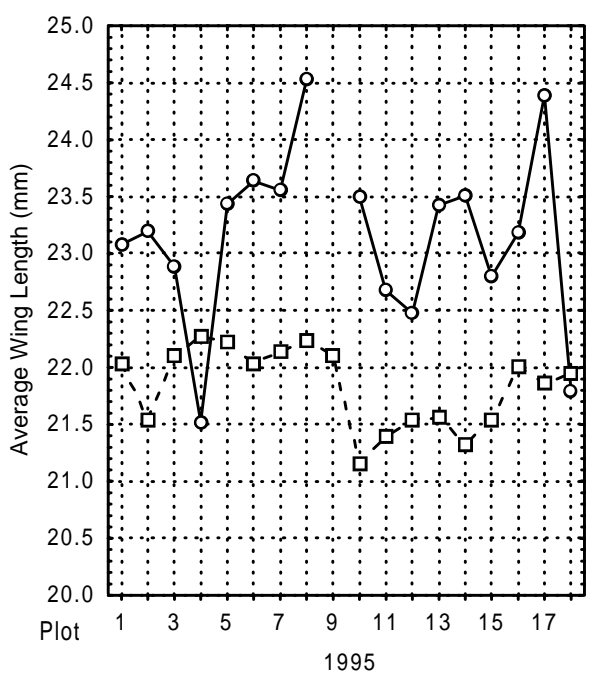

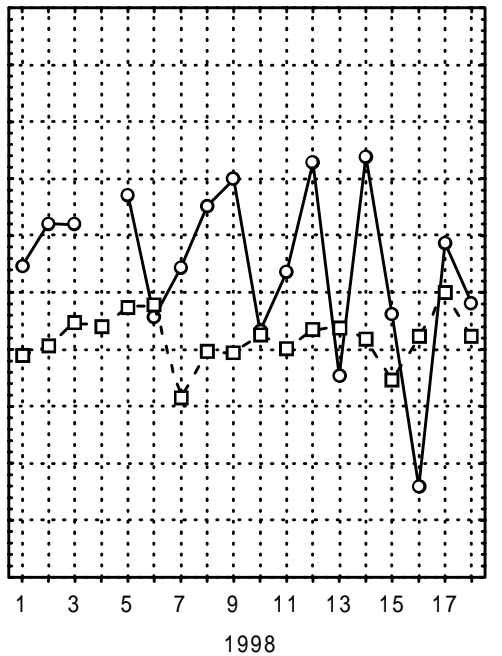

Reference:

(plots 2, 4, 8, 12, 14, 16)

Bt:

(plots 3, 5, 7, 11, 15, 18)

Gypchek:

(plots 1, 6, 9, 10, 13,17)

$\rightarrow$ Females

$-\square$ - Males
Reference:

(plots 2, 4, 8, 12, 14, 16)

Bt:

(plots 3, 5, 7, 11, 15, 18)

Gypchek:

(plots 1, 6, 9, 10, 13, 17)

Figure 54. Female and male adult counts and wing lengths (1995 \& 1998). Graphs illustrate average plot counts and wing lengths of female and male Halysidota tesselaris collected during the 1995 pretreatment and 1998 treatment years from the George Washington (plots 1-9) and Monongahela (plots 10-18) National Forests. 


\section{Achatia distincta}

A total of 56 larva and 148 adults was collected during 1995-1998. Larvae were sparsely collected throughout the first eight weeks of the sampling season, with a maximum average of 1.13 larvae collected during week 3. The first graph in Figure 55 illustrates a significant interaction for YEAR x TREATMENT $(\mathrm{P}=0.043)$. Larvae collected during the treatment years from GC plots were significantly more abundant than those collected from $B t$ plots during treatment years. The larvae were also more abundant in the GC plots during treatment years than in the GC plots during pretreatment years. The second graph in Figure 54 illustrates the adult trends. Significant differences were not indicated.

Only 51 individuals were measured during 1995 \& 1998 for wing length. Females averaged 16.29 $\mathrm{mm}$ and males averaged $16.22 \mathrm{~mm}$. An ANOVA was not conducted due to insufficient numbers from several categories. 

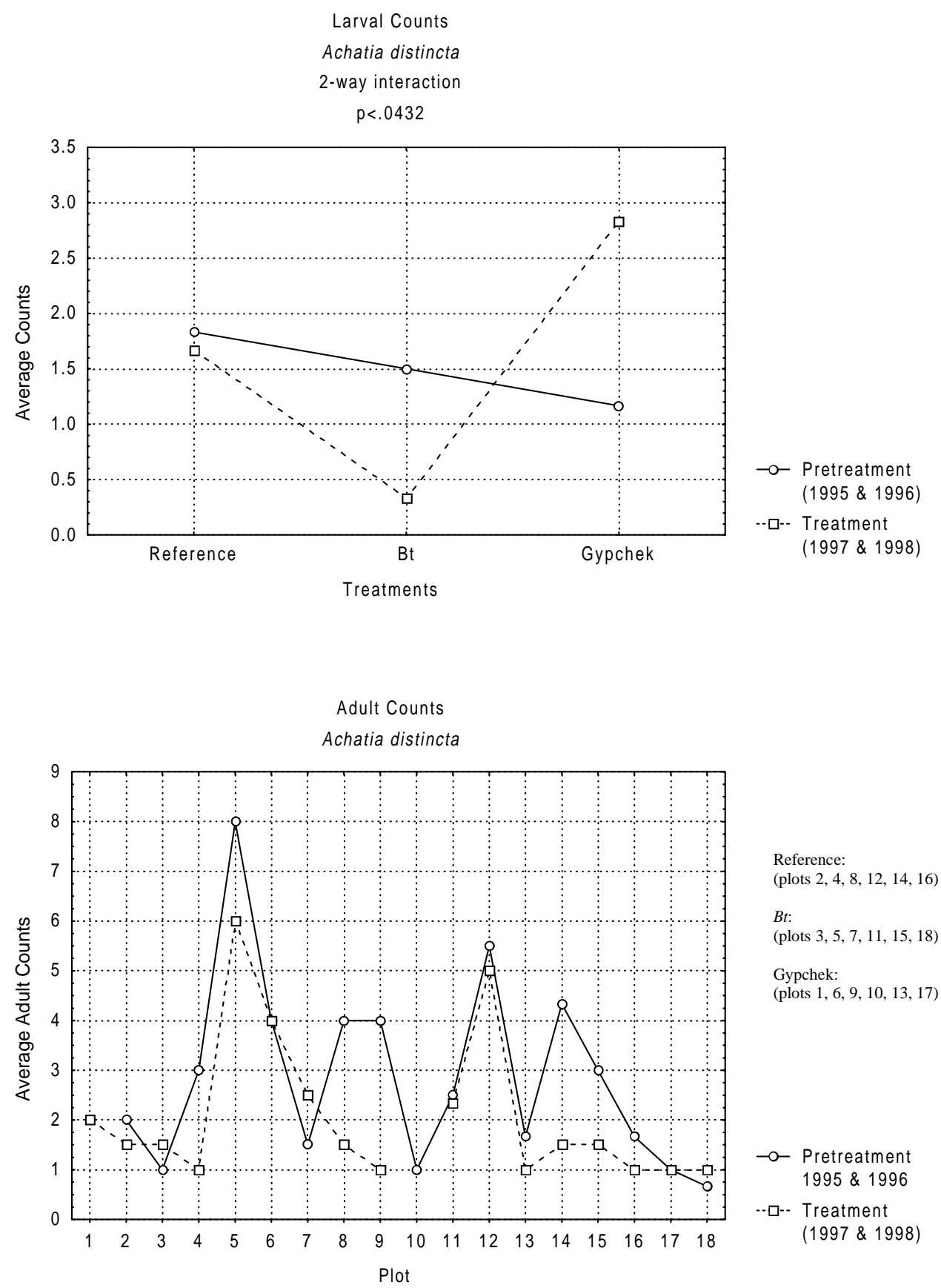

Figure 55. Larval interaction and adult counts (1995-1998). The first graph illustrates the Achatia distincta larval count interaction between treatment categories and pretreatment/treatment years. The second graph illustrates average plot counts for adults collected during pretreatment and treatment years from the George Washington (plots 1-9) and Monongahela (plots 10-18) National Forests. 


\section{Polia latex}

A total of 1,632 larvae and 1,897 adults was collected during 1995-1998. The graphs in Figure 56 illustrate average weekly catches. Larvae typically appeared after week 4. Adults were captured throughout the sampling period with peak abundance in catches near mid-season. The first and second graphs of Figure 57 illustrate the larval and adult trends relative to the plots, respectively. The ANOVA indicated that larvae and adults collected during treatment years were more abundant in catches than during pretreatment years $(\mathrm{P}=0.001$ and $\mathrm{P}<0.001$, respectively). Significantly more moths were captured from the MON than from the $\mathrm{GW}(\mathrm{P}=0.014)$.

A total of 416 female and 661 male moths was identified from 1995 and 1998 catches (see the first graph in Figure 58). The ANOVA indicated that the SEX difference was significant $(\mathrm{P}=0.022)$. Significantly more moths were collected from 1998 than from 1995 ( $\mathrm{P}<0.001)$. A total of 1,053 moths was measured for wing length (see the second graph in Figure 58). Group averages were substituted in 4 cases where females appeared in insufficient numbers. The ANOVA indicated that SEX, YEAR, and FOREST main effects were significant $(\mathrm{P}<0.001, \mathrm{P}=0.004$, and $\mathrm{P}=0.001$, respectively). Females were significantly larger than males. Moths were significantly smaller during treatment years in the MON forest when compared to the GW.

A total of 328 larvae was weighed. Larval averages collected from the GW were significantly heavier than those collected from the MON $(\mathrm{P}=0.007)$. A total of 190 larvae successfully pupated in the laboratory. As with larvae, resulting pupae were heavier for the $\mathrm{GW}$ than for the $\mathrm{MON}(\mathrm{P}=0.005)$. The first and second graphs in Figure 59 illustrate larval and pupal trends, respectively. 
Weekly Larval Counts

Polia latex
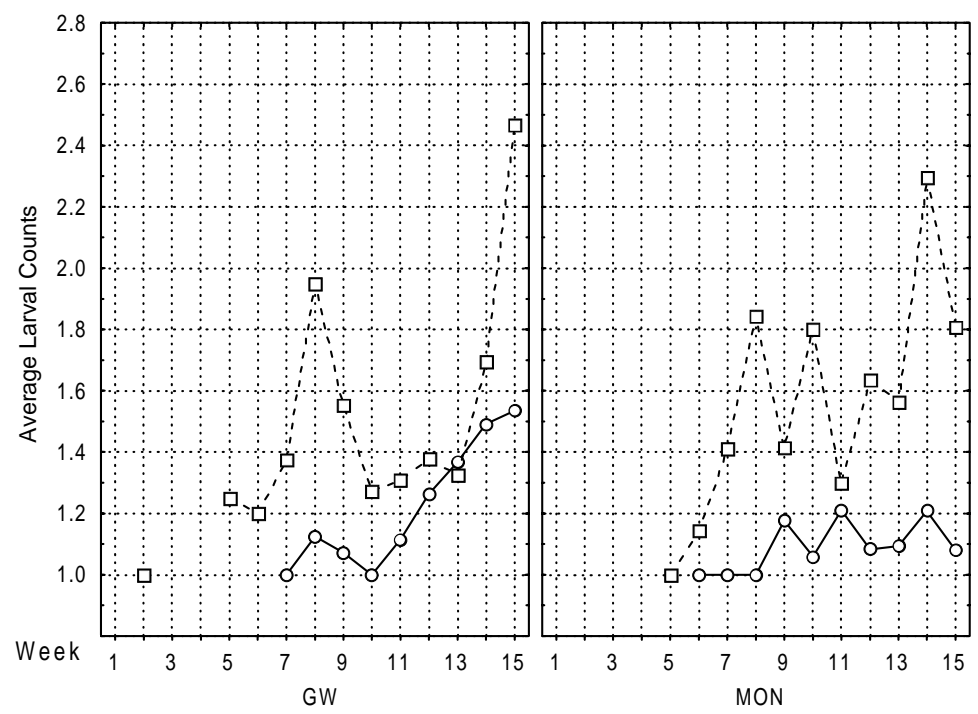

-o- Pretreatment

(1995 \& 1996)

-- $\square$-- Treatment

(1997 \& 1998)

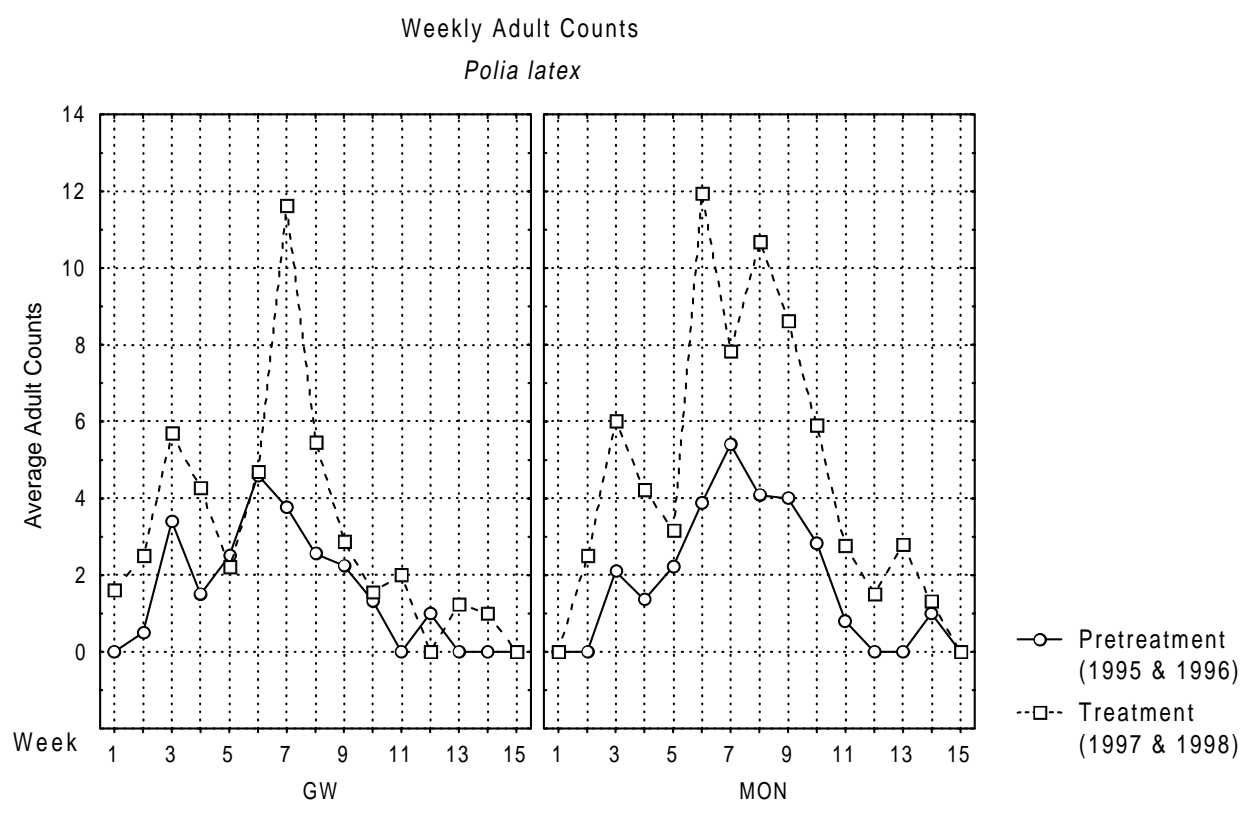

Figure 56. Weekly larval and adult counts (1995-1998). Graphs illustrate average weekly larval and adult counts for Polia latex collected during pretreatment and treatment years from the George Washington (GW) and Monongahela (MON) National Forests. 

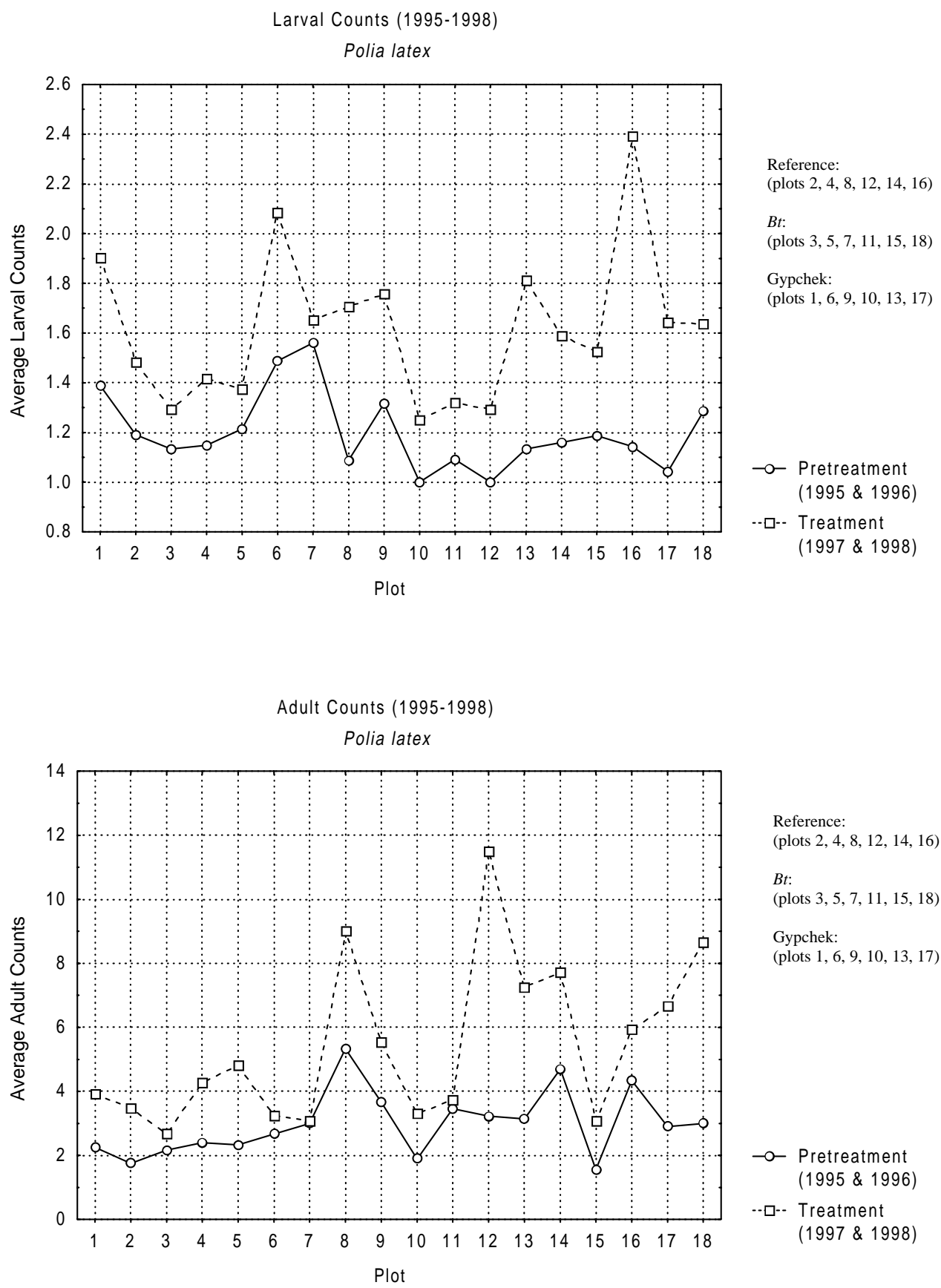

Reference:

(plots 2, 4, 8, 12, 14, 16)

Bt:

(plots 3, 5, 7, 11, 15, 18)

Gypchek:

(plots $1,6,9,10,13,17$ )

- - Pretreatment (1995 \& 1996)

- $\square$-- Treatment

(1997 \& 1998)

Figure 57. Larval and adult counts (1995-1998). Graphs illustrate average plot counts of larval and adult Polia latex collected during pretreatment and treatment years from the George Washington (plots 1-9) and Monongahela (plots 10-18) National Forests. 

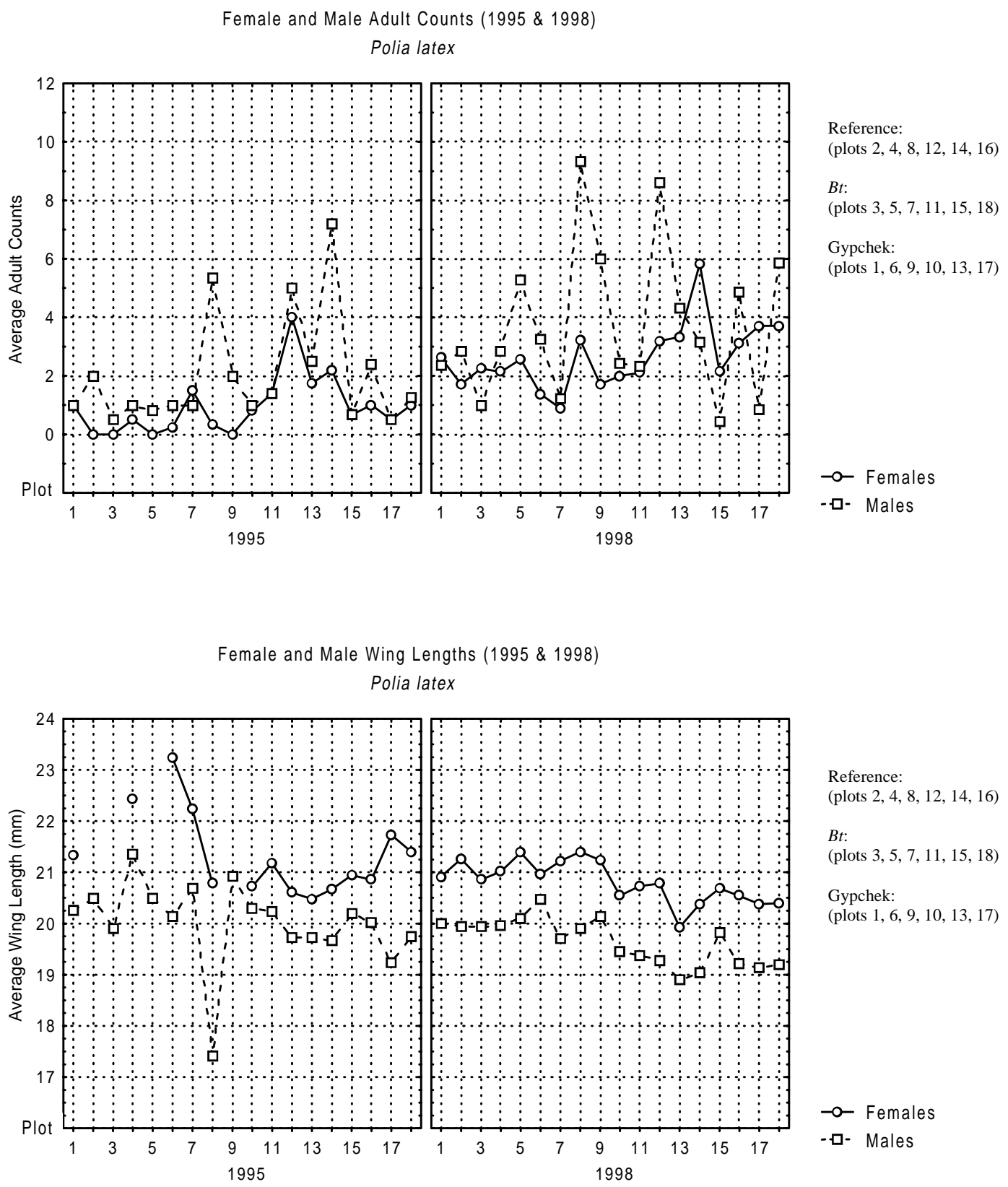

Figure 58. Female and male adult counts and wing lengths (1995 \& 1998). Graphs illustrate average plot counts of female and male Polia latex collected during the 1995 pretreatment and 1998 treatment years from the George Washington (plots 1-9) and Monongahela (plots 10-18) National Forests. 

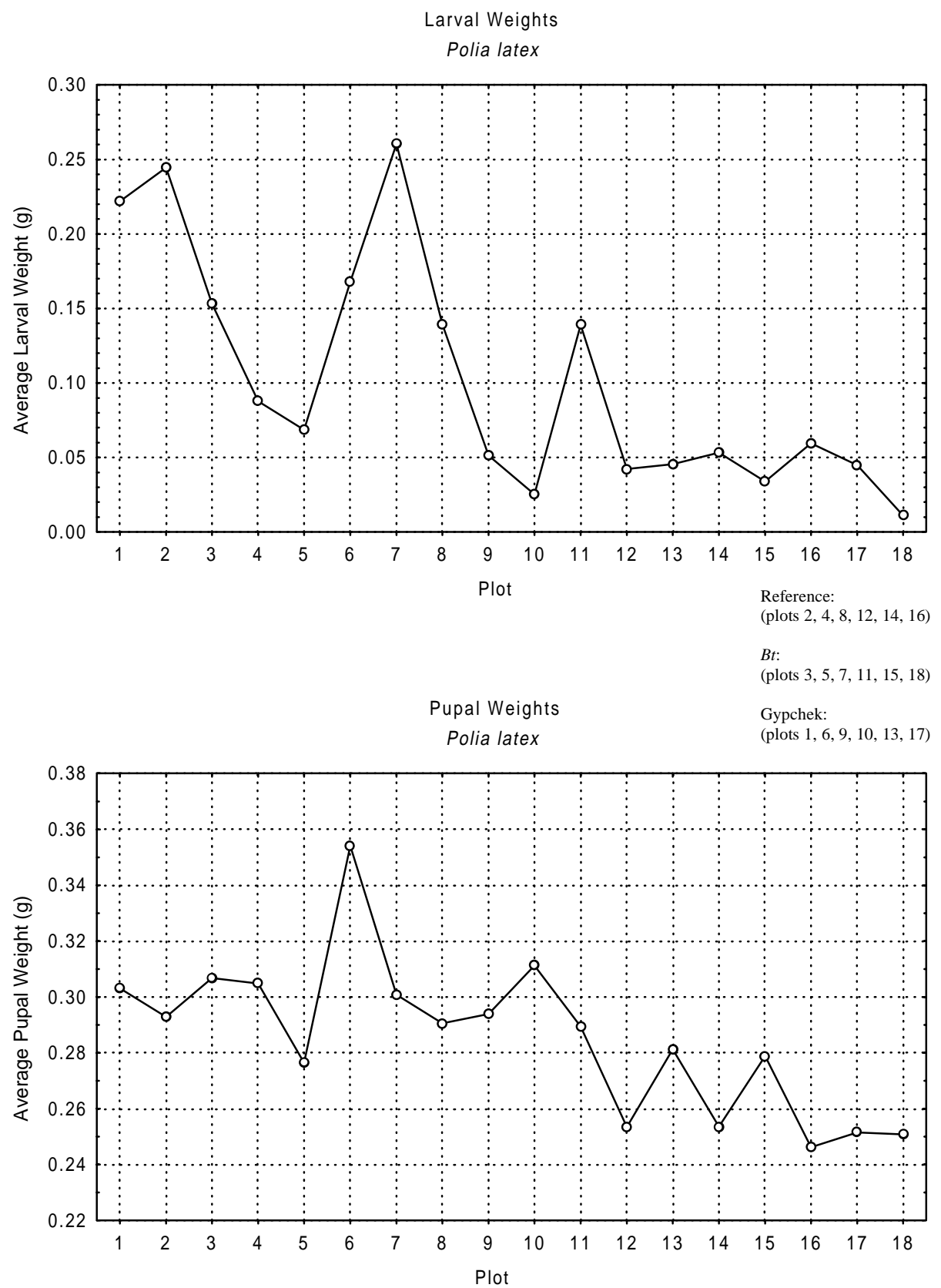

Figure 59. Larval and pupal weights (1997-1998). The first graph illustrates average larval weights of Polia latex collected during treatment years from each plot. The second graph illustrates average pupal weights resulting from rearing the larvae in the laboratory. George Washington National Forest plots are 1-9; Monongahela National Forest Plots are 10-18. 


\section{Morrisonia confusa}

A total of 323 larvae and 1,738 adults was collected during 1995-1998. The graphs in Figure 60 illustrate the average weekly trends. Larvae were captured throughout the sampling season beginning after week 2. Adults showed an early season peak in abundance between week 1-6.

Trends for larvae and adults relative to the plots are illustrated as the first and second graphs in Figure 61. The ANOVA indicated that YEAR, FOREST, and YEAR x FOREST were significantly different for the larvae $(\mathrm{P}=0.001, \mathrm{P}=0.008$, and $\mathrm{P}=0.007$, respectively). Most larvae were collected during the treatment years from the MON. The adults showed significant differences for YEAR, FOREST, and FOREST x TREATMENT ( $\mathrm{P}=0.001, \mathrm{P}=0.004$, and $\mathrm{P}=0.014$, respectively). More moths were collected during treatment years and more were collected from the MON than from the GW. Significantly more moths were collected from Ref plots in the MON than from all but one other statistical group. The difference between $B t$ plots in the GW and Ref plots in the GW were not significant. The interaction is illustrated in the third graph in Figure 61.

A total of 563 female and 719 male moths was identified from 1995 and 1998 catches. The first graph in Figure 62 illustrates the trends. The ANOVA indicated trends similar to what was observed for the two pretreatment and treatment years, combined. The effects for YEAR, FOREST, and YEAR $x$ FOREST were significant $(\mathrm{P}=0.001, \mathrm{P}=0.002$, and $\mathrm{P}=0.044$, respectively). Differences relative to SEX were not observed.

A total of 587 female and 683 male moths was measured for wing length. Group averages were substituted in two cases where insufficient numbers of intact males were collected. The main effects for SEX, YEAR, and FOREST, were significant $(\mathrm{P}<0.001, \mathrm{P}<0.001$, and $\mathrm{P}=0.026$, respectively). Females were significantly larger than males. Larger moths were collected during pretreatment years. Larger 
moths were collected from the GW than from the MON. The second graph in Figure 62 illustrates the trends. 

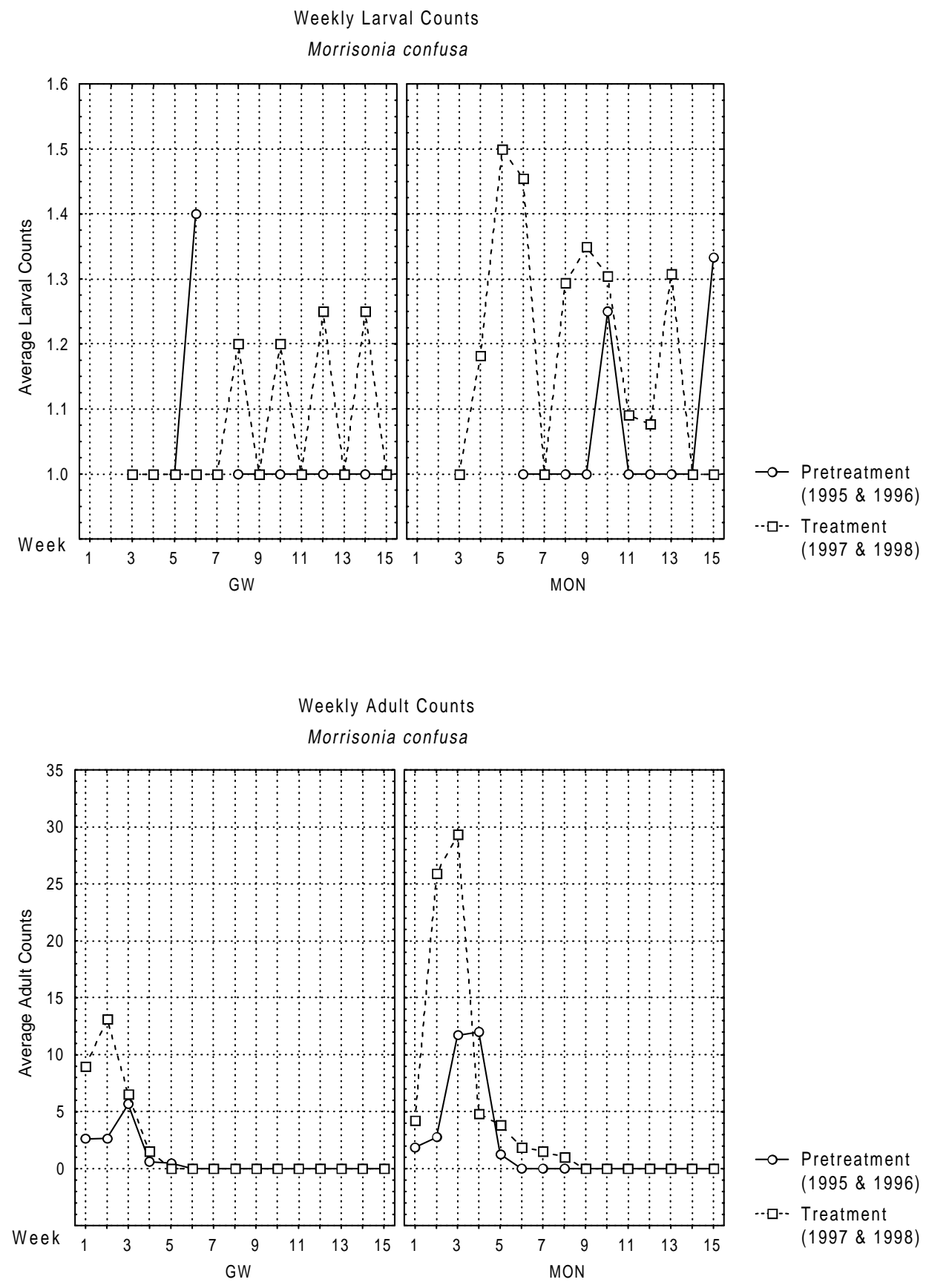

Figure 60. Weekly larval and adult counts (1995-1998). Graphs illustrate average weekly larval and adult counts of Morrisonia confusa collected during pretreatment and treatment years from the George Washington (GW) and Monongahela (MON) National Forests. 


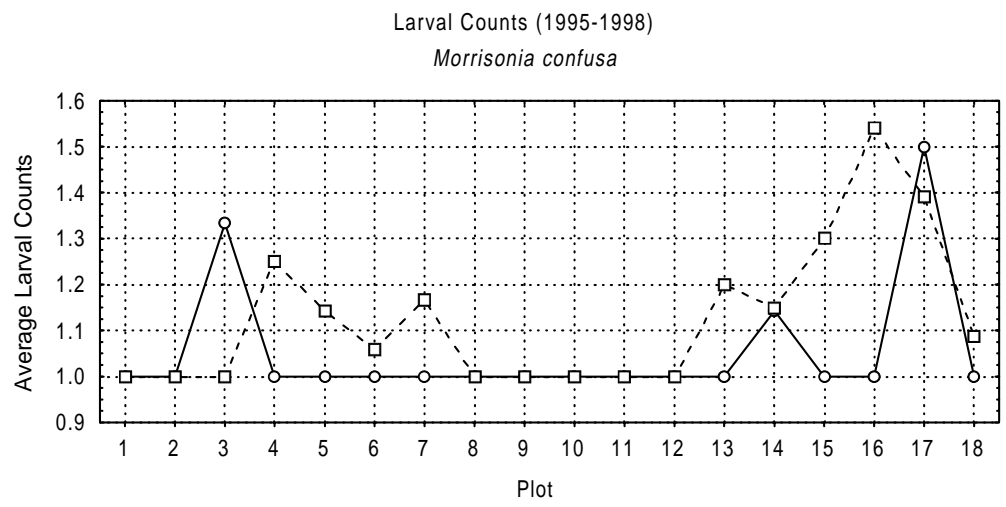

Reference:

(plots 2, 4, 8, 12, 14, 16)

$B t$ :

(plots 3, 5, 7, 11, 15, 18)

Gypchek:

(plots 1, 6, 9, 10, 13, 17)

-o- Pretreatment

(1995 \& 1996)

- $\square$ - Treatment

(1997 \& 1998)

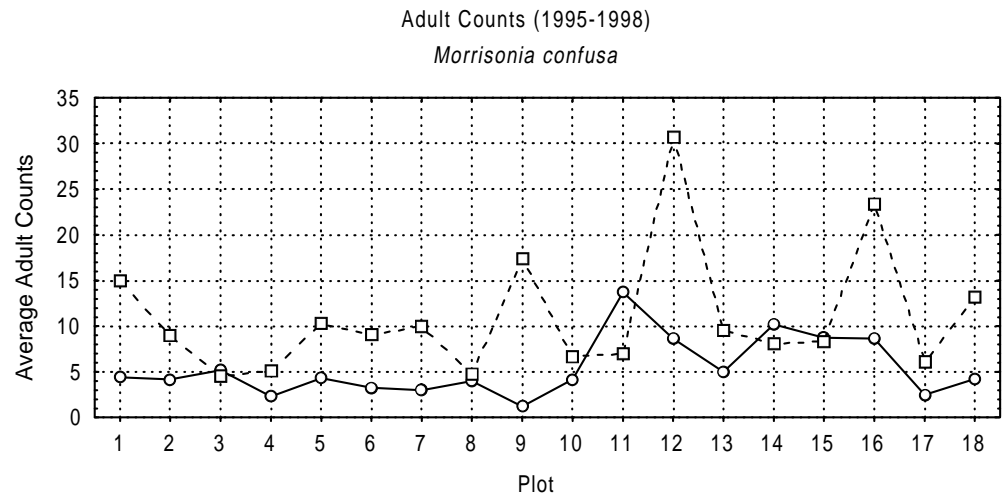

Reference:

(plots 2, 4, 8, 12, 14, 16)

Bt:

(plots 3, 5, 7, 11, 15, 18)

Gypchek:

(plots 1, 6, 9, 10, 13, 17)

-o- Pretreatment

(1995 \& 1996)

--ם- Treatment

(1997 \& 1998)

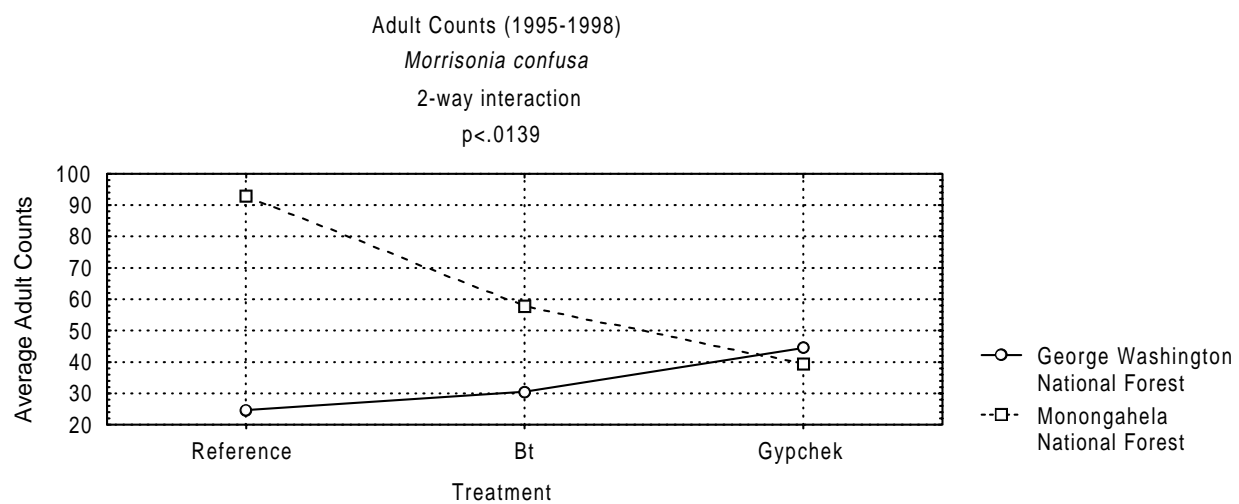

Figure 61. Larval and adult counts with adult interaction (1995-1998). The first two graphs illustrate average plot counts for larval and adult Morrisonia confusa collected during pretreatment and treatment years from the George Washington (plots 1-9) and Monongahela (plots 10-18) National Forests. The third graph illustrates the adult count interaction between treatment categories and forests. 
Morrisonia confusa
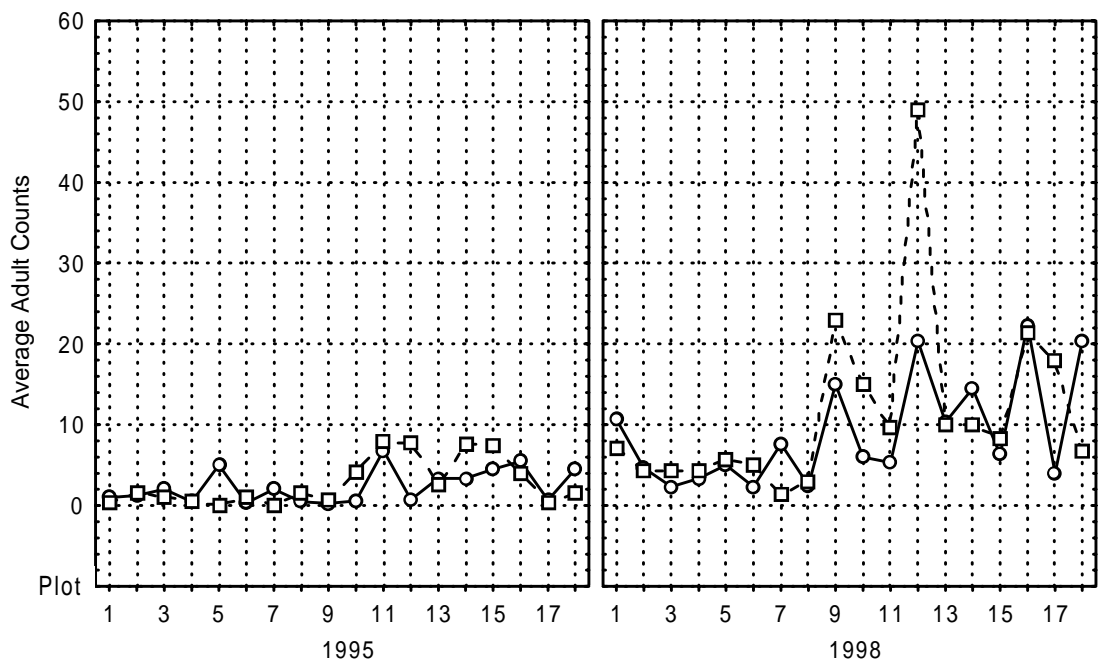

Reference:

(plots 2, 4, 8, 12, 14, 16)

$B t:$

(plots 3, 5, 7, 11, 15, 18)

Gypchek:

(plots 1, 6, 9, 10, 13, 17)

-o- Females

- $\square$ - Males
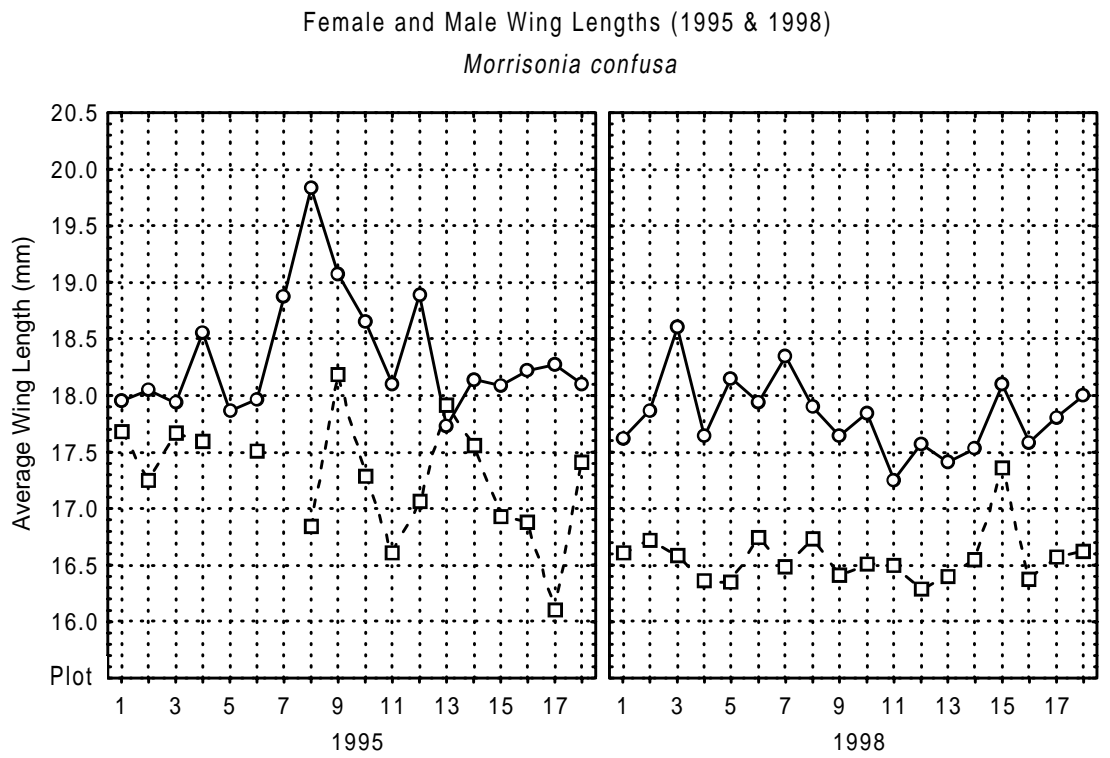

Reference:

(plots 2, 4, 8, 12, 14, 16)

$B t$ :

(plots 3, 5, 7, 11, 15, 18)

Gypchek:

(plots 1, 6, 9, 10, 13, 17)

- - Females

- $\square$ - Males

Figure 62. Female and male adult counts and wing lengths (1995 \& 1998). Graphs illustrate average plot counts and wing lengths for female and male Morrisonia confusa collected during the 1995 pretreatment and 1998 treatment years from the George Washington (plots 1-9) and Monongahela (plots 10-18) National Forests. 


\section{Allotria elonympha}

A total of 9 larvae and 1,013 adults was collected during 1995-1998. The few larvae collected were captured during the last 4 weeks of the sampling seasons. They were not independently analyzed. Weekly trends for adult moths are presented as the first graph in Figure 63. The majority of moths were collected prior to week 10. The second graph in Figure 63 illustrates trends relative to the plots. The ANOVA indicated significant differences for YEAR, FOREST, and YEAR x FOREST ( $\mathrm{P}<0.001$ in each instance). More moths were collected during treatment years. The GW provided significantly more moths than the MON. The interaction indicated that more moths were collected from the GW during treatment years and that the MON yielded fewer moths than the GW during both sets of years; however, the differences within the MON between pretreatment and treatment years were not significant.

A total of 93 female and 438 male moths was identified from 1995 and 1998 catches (see third graph in Figure 63). Due to numerous gaps in the grouping data, an ANOVA was not conducted. The graph indicates that the vast majority of moths were collected from the GW and that males were collected more frequently than females. As most moths were intact, a student's t-test was conducted on wing length measurements which indicated that females were significantly larger than males (19.491 and19.239 mm, respectively; $\mathrm{P}=0.016)$. 

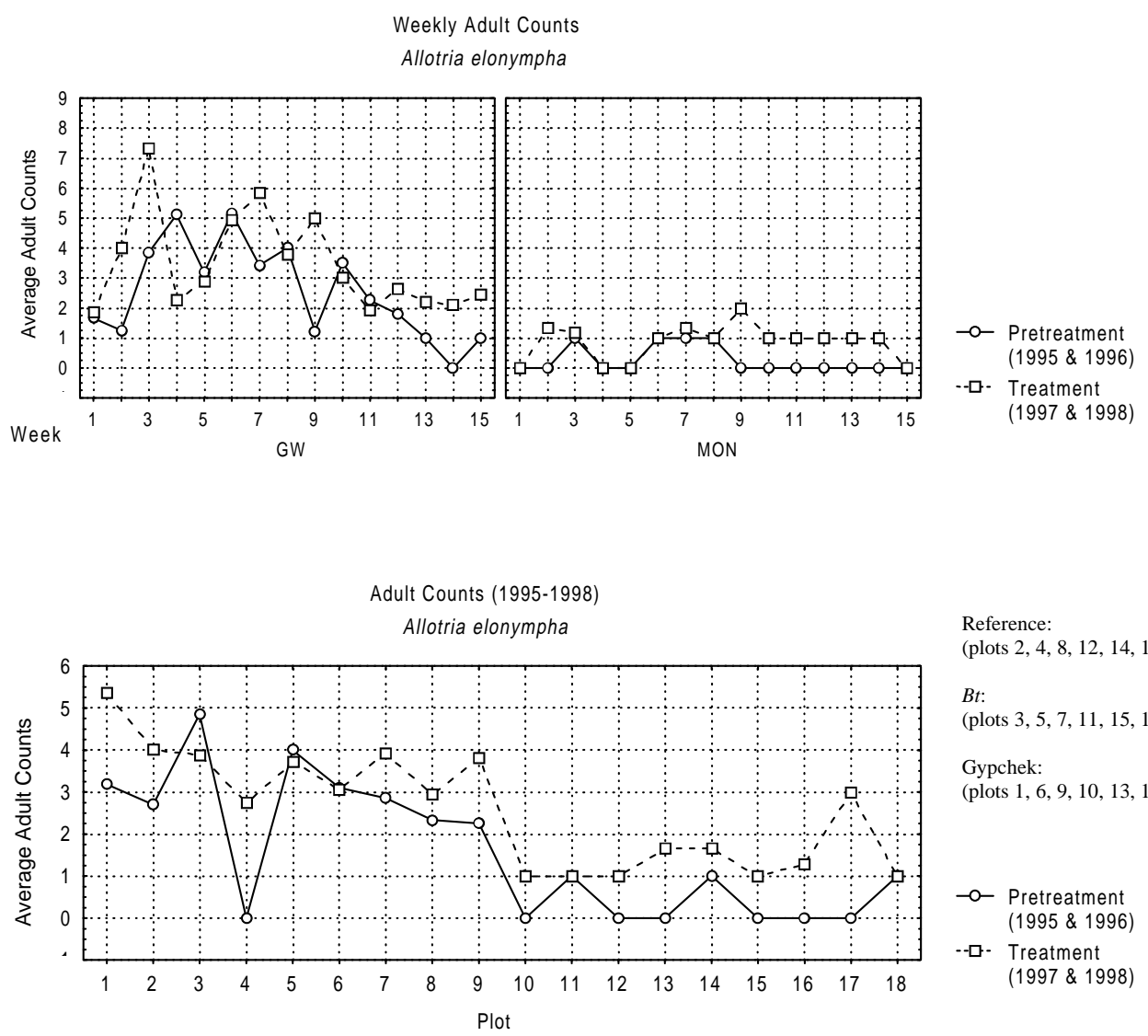

Reference: (plots 2, 4, 8, 12, 14, 16)

$B t$ :

(plots 3, 5, 7, 11, 15, 18)

Gypchek:

(plots $1,6,9,10,13,17$ )

-o- Pretreatment

(1995 \& 1996)

--ם- Treatment

(1997 \& 1998)

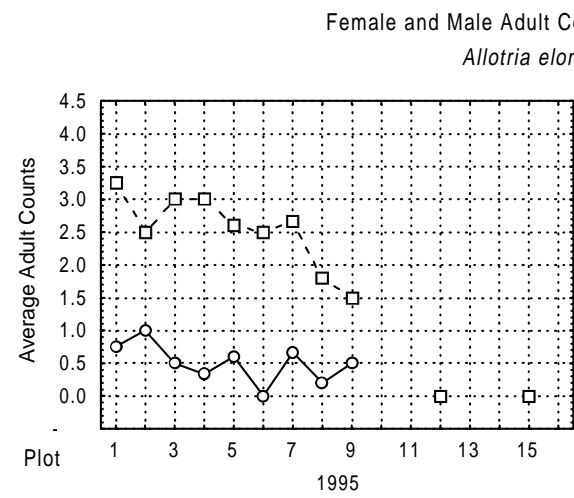

Counts (1995 \& 1998)

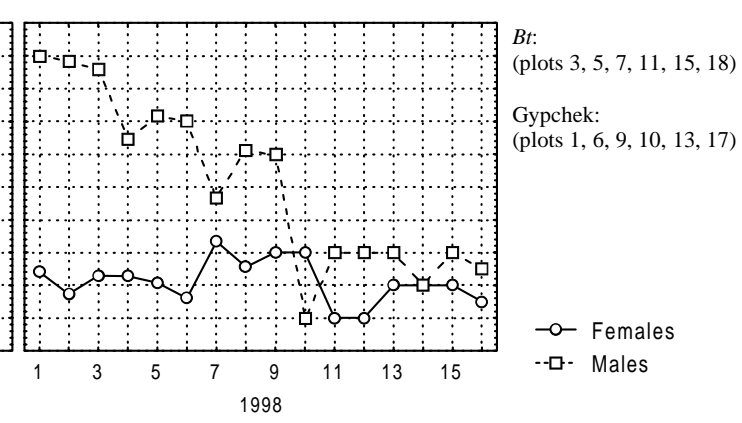

Figure 63. Adult counts (1995-1998; 1995 \& 1998). The first two graphs illustrate average weekly and average plot counts of Allotria elonympha adults collected during pretreatment and treatment years from the George Washington (GW; plots 1-9) and the Monongahela (MON; plots 10-18) National Forests. The third graph illustrates the average plot counts for males and females collected during the 1995 pretreatment and 1998 treatment years. 


\section{Baileya opthalmica}

A total of 128 larvae and 1,309 adults was collected during 1995-1998. The weekly catches for larvae and adults appear as the first and second graphs in Figure 64, respectively. Larvae were collected primarily after week 7 during the sampling seasons. Adults were captured primarily within the first 10 weeks of the sampling season.

The first and second graphs in Figure 65 illustrate plot trends for larvae and adults, respectively. The ANOVA indicated that the FOREST main effect was significant for larvae $\mathrm{P}=0.002$ ). More larvae were collected from the MON than from the GW. Interactions for YEAR x FOREST and for YEAR x FOREST $x$ TREATMENT were also significant $(\mathrm{P}=0.002$ and $\mathrm{P}=0.042$, respectively). The first interaction indicated that for both sets of years, MON moths were more abundant. The 3-way interaction indicated that treatment year catches in the $B t$ and GC plots were significantly higher in the MON when compared to the GW. Adults showed significant differences for YEAR, FOREST, and YEAR x FOREST $(\mathrm{P}=0.002, \mathrm{P}=0.001$, and $\mathrm{P}=0.002$, respectively). More moths were collected during treatment years from the MON than any other statistical group.

A total of 295 female and 486 male moths were identified for 1995 and 1998 catches (see first graph in Figure 66). Main effects for SEX, YEAR, and FOREST, were indicated $(\mathrm{P}=0.002, \mathrm{P}=0.003$, and $\mathrm{P}=0.002$, respectively). Significant interactions were identified for SEX x YEAR, SEX x FOREST, YEAR x FOREST, SEX x YEAR x FOREST, and SEX x FOREST x TREATMENT $(\mathrm{P}=0.008, \mathrm{P}=0.035, \mathrm{P}=0.003$, $\mathrm{P}=0.047$, and $\mathrm{P}=0.032$, respectively). A summary of these interactions shows more males and more females were collected during 1998 from the MON than from the GW or when compared with 1995 moth data for either sex or forest. Males were significantly more abundant than females in the 1998 MON catches. Although all treatment assignments showed increases in counts from 1995 to 1998, only males in GC plots showed a significant increase during 1998 when compared to males and females collected from the GC plots during 1995. 
For wing length comparisons, group averages were substituted in 4 cases where insufficient numbers of intact moths were collected. A total of 295 females and 486 males were measured. Females were significantly larger than males $(\mathrm{P}=0.021)$. Moths collected during 1998 were smaller $(\mathrm{P}<0.001)$ than those collected during 1995 (see second graph in Figure 66). 

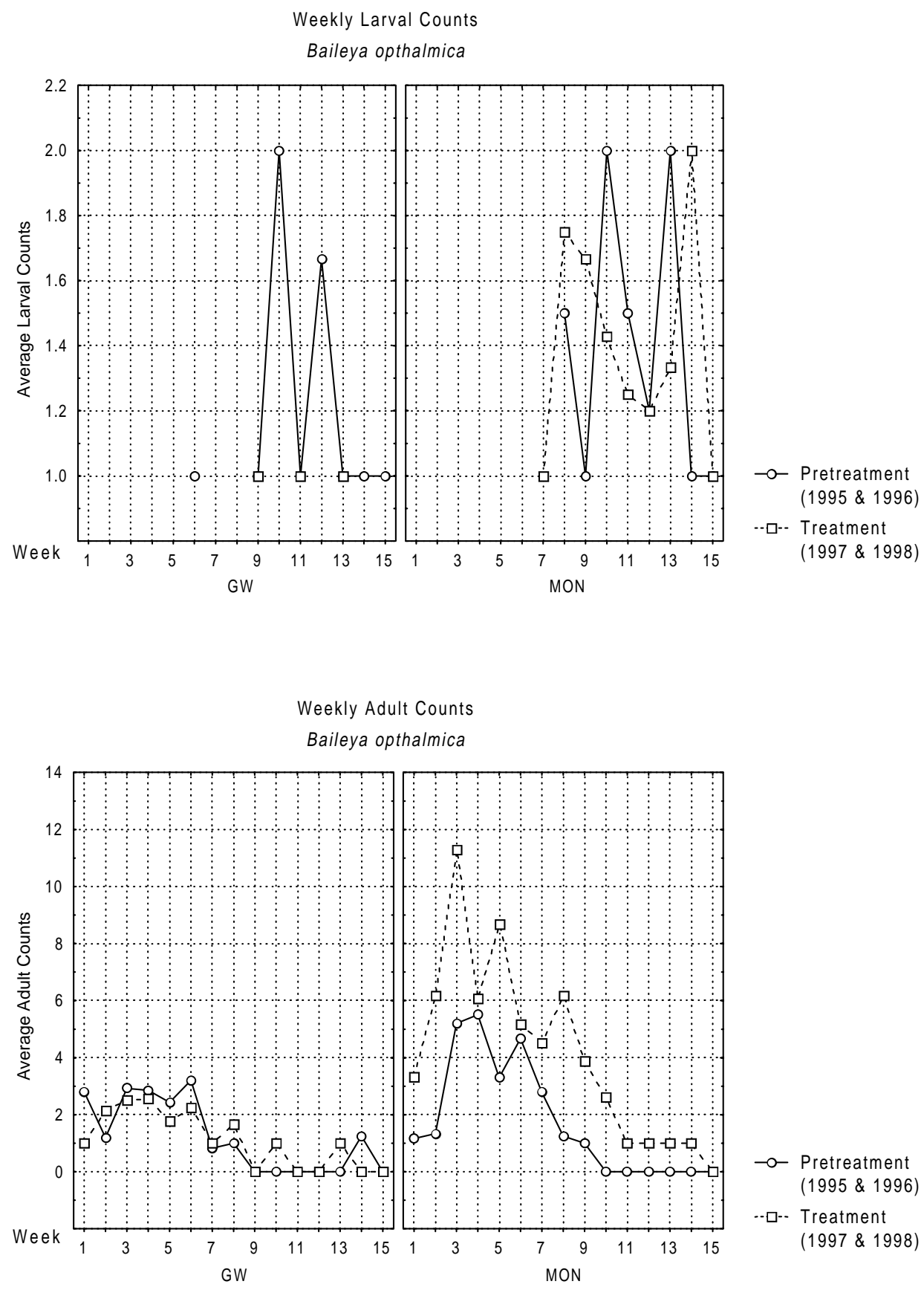

Figure 64. Weekly larval and adult counts (1995-1998). Graphs illustrate average weekly larval and adult counts for Baileya opthalmica collected during pretreatment and treatment years from the George Washington (GW) and Monongahela (MON) National Forests. 


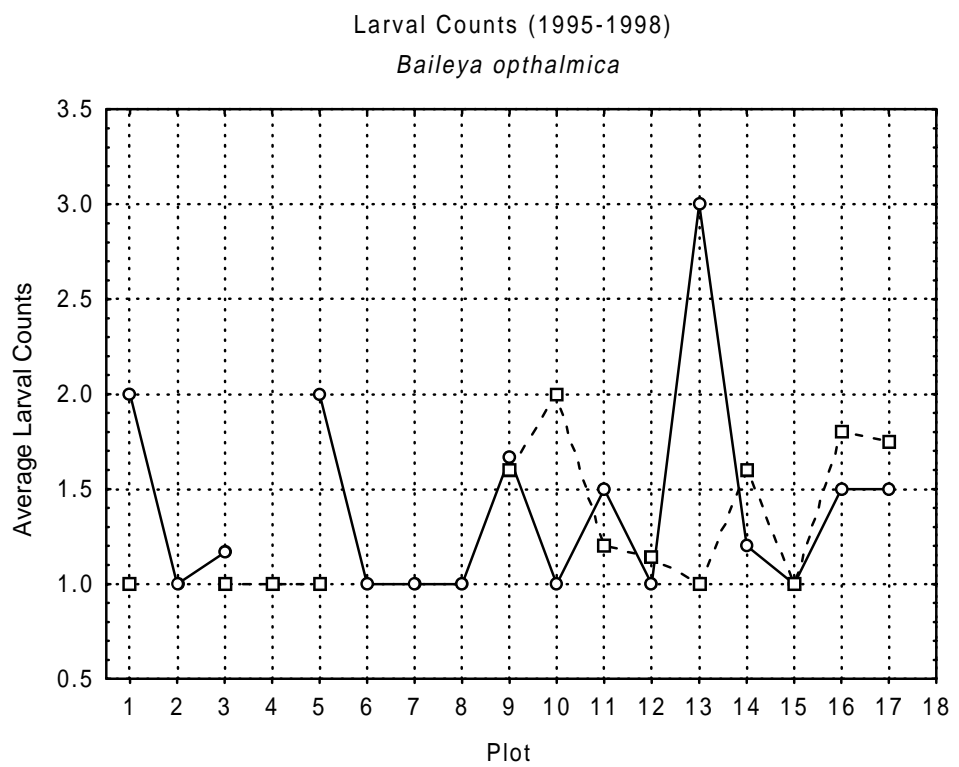

Reference:

(plots 2, 4, 8, 12, 14, 16)

Bt:

(plots $3,5,7,11,15,18$ )

Gypchek:

(plots 1, 6, 9, 10, 13, 17)

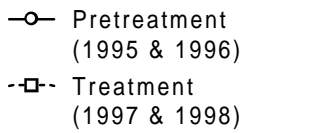

Adult Counts (1995-1998)

Baileya opthalmica

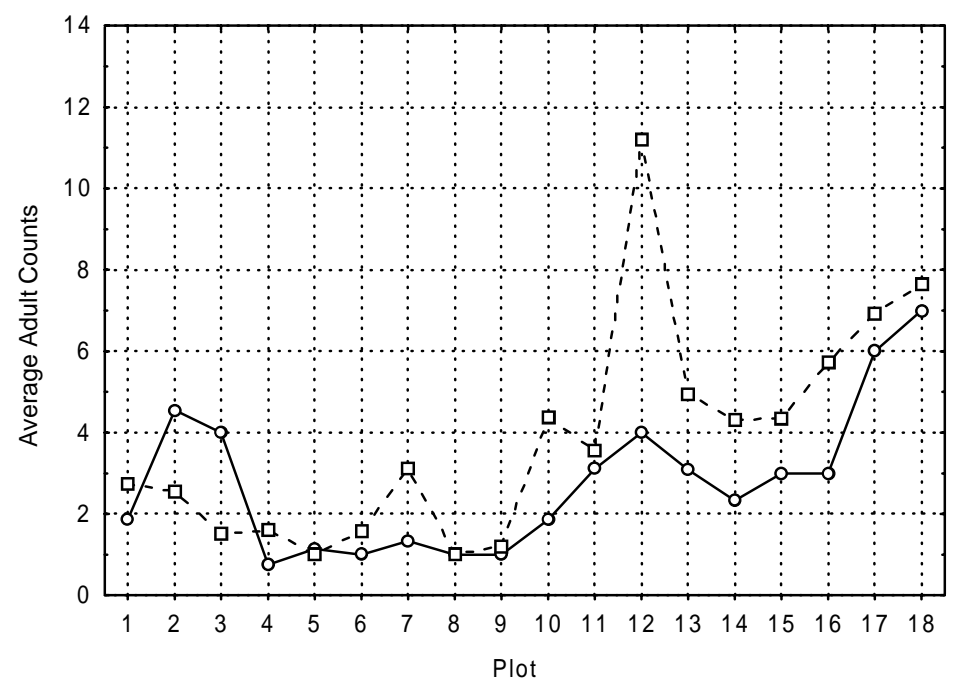

Reference:

(plots $2,4,8,12,14,16)$

Bt:

(plots 3, 5, 7, 11, 15, 18)

Gypchek:

(plots 1, 6, 9, 10,13,17)

- - Pretreatment

(1995 \& 1996)

- ㅁ- Treatment

(1997 \& 1998)

Figure 65. Larval and adult counts (1995-1998). Graphs illustrate average plot counts for larval and adult Baileya opthalmica collected during pretreatment and treatment years from the George Washington (plots 1-9) and Monongahela (plots 10-18) National Forests. 

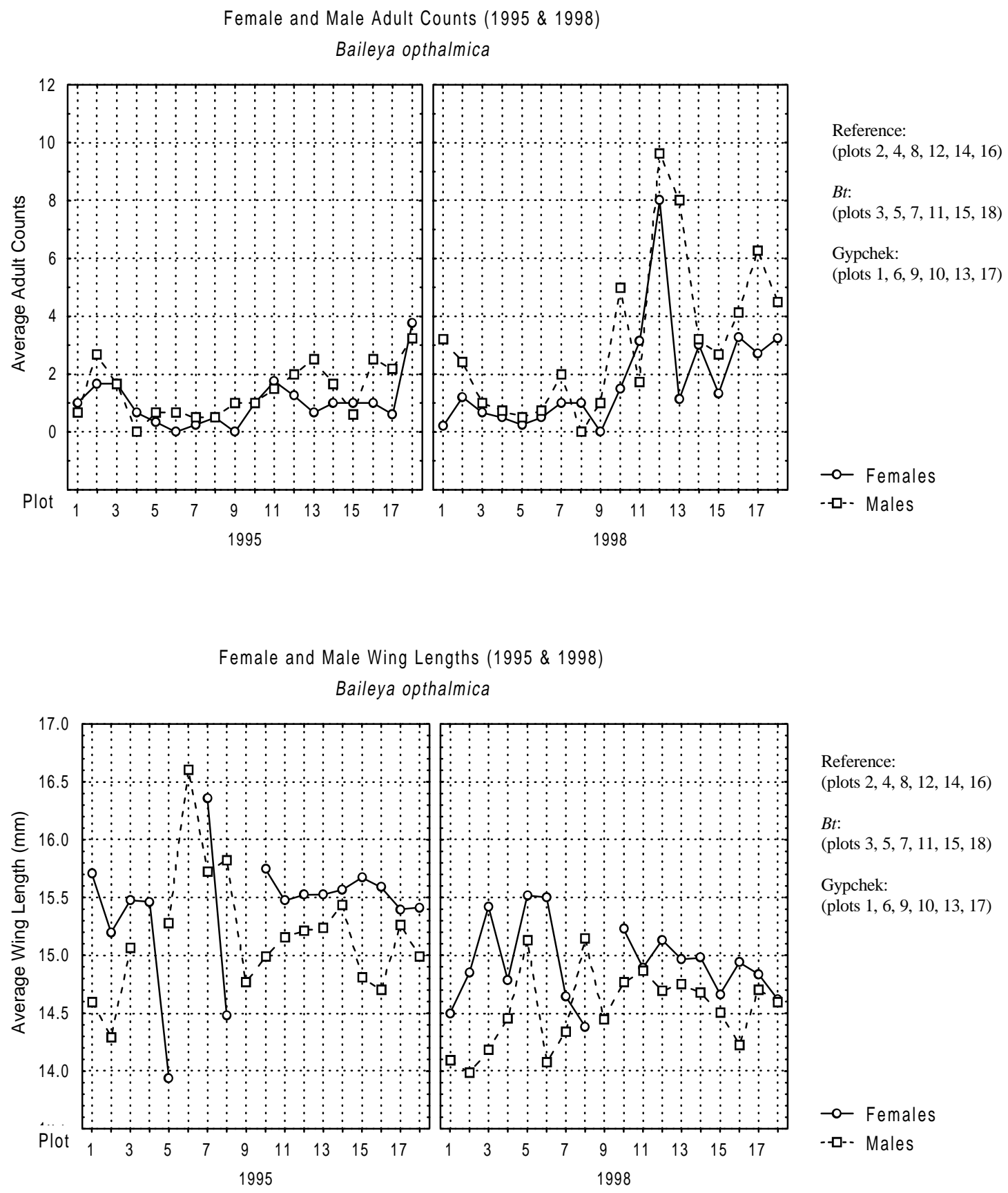

Reference:

(plots 2, 4, 8, 12, 14, 16)

$B t$ :

(plots $3,5,7,11,15,18$ )

Gypchek:

(plots 1, 6, 9, 10, 13, 17)

-o- Females

- - - Males

Figure 66. Female and male adult counts and wing lengths (1995 \& 1998). Graphs illustrate average plot counts and wing lengths of female and male Baileya opthalmica collected during the 1995 pretreatment and 1998 treatment years from the George Washington (plots 1-9) and Monongahela (plots 10-18) National Forests. 


\section{Acronicta ovata}

A total of 2,307 larvae and 39,084 adults was collected during 1995-1998. The first and second graphs in Figure 67 illustrate average weekly trends for larvae and adults, respectively. Most larvae and adults appeared after week 7 .

The trends for larvae and adults are presented as the first and second graphs in Figure 68, respectively. The ANOVA indicated that significantly more larvae were collected from the MON than from the GW ( $\mathrm{P}=0.016)$. For adults, a significant effect for YEAR was indicated $(\mathrm{P}=0.015)$, with more moths captured during treatment years than during pretreatment years.

A total of 2,988 females and 9,952 males were identified from 1995 and 1998 catches (see first graph in Figure 69). In this case, the ANOVA indicated the SEX difference to be significant $(\mathrm{P}=0.001)$. A total of 10, 018 of these moths were measured for wing length (see second graph in Figure 69). Females were significantly larger than males $(\mathrm{P}<0.001)$. A significant FOREST effect and a SEX $x$ YEAR $x$ FOREST interaction were also discerned $(\mathrm{P}=0.011$ and $\mathrm{p}=0.049$, respectively). Moths collected from the $\mathrm{GW}$ had longer wings length than those collected from the MON. In all cases, females were larger than males; however, females collected from the MON during 1998 were significantly smaller than those collected from the MON during 1995 and from those collected from the GW during either year. Males collected during 1995 from the GW were larger than 1995 MON males. Yearly differences for males collected within each forest were not significant. 

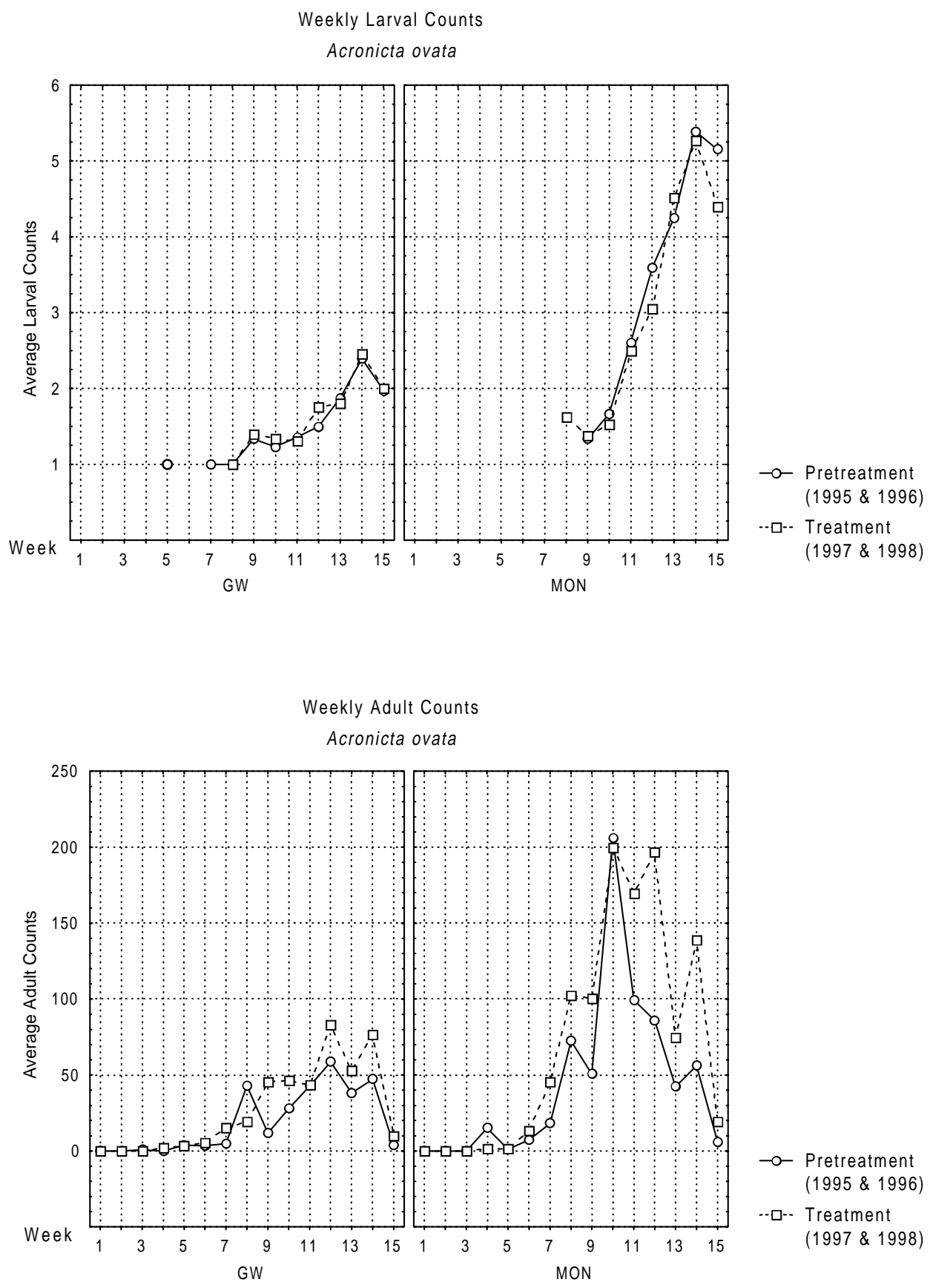

eekly Adult Counts

Figure 67. Weekly larval and adult counts (1995-1998). Graphs illustrate average weekly larval and adult counts of Acronicta ovata collected during pretreatment and treatment years from the George Washington (GW) and Monongahela (MON) National Forests. 

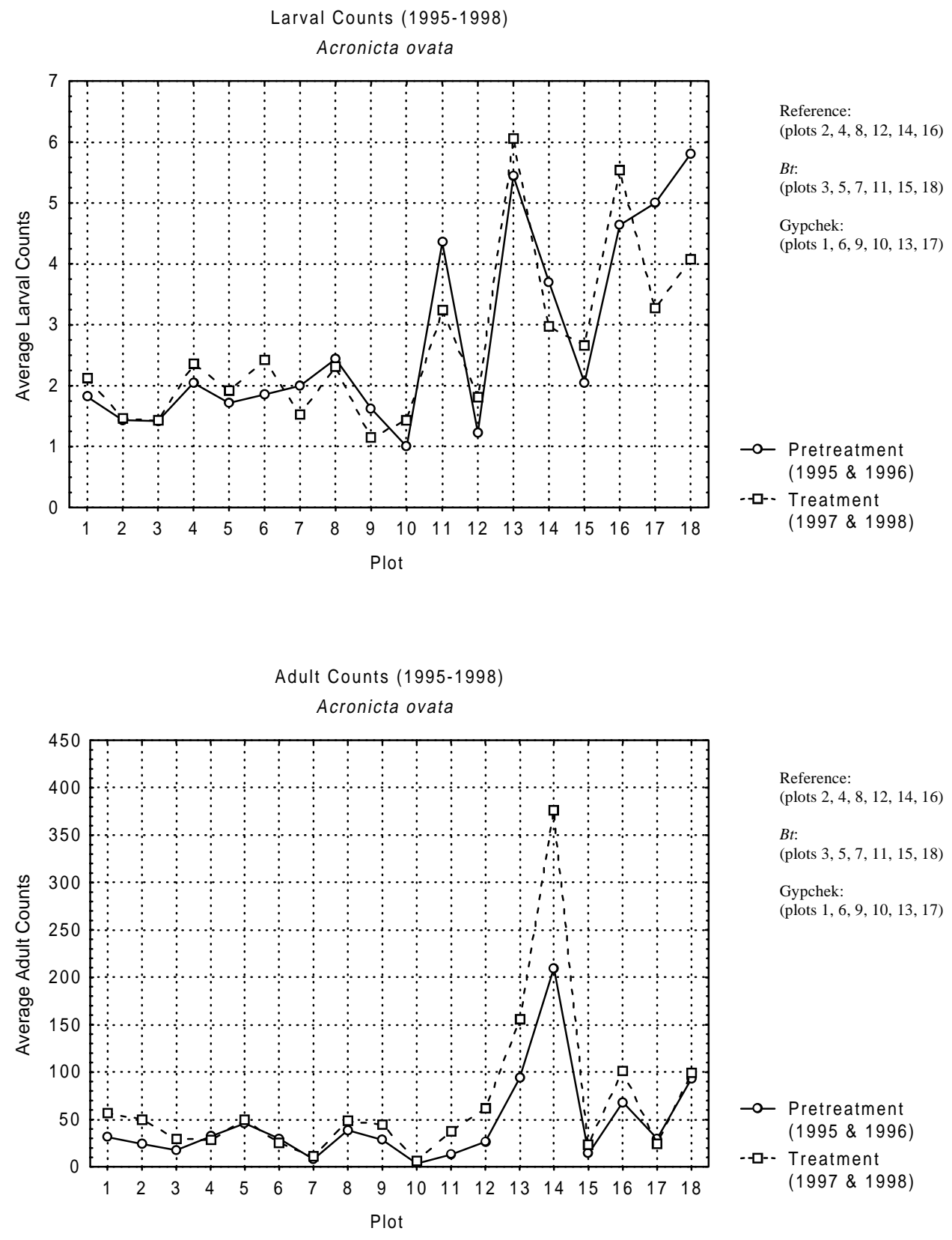

Figure 68. Larval and adult counts (1995-1998). Graphs illustrate average plot counts for larval and adult Acronicta ovata collected during pretreatment and treatment years from the George Washington (plots 19) and Monongahela (plots 10-18) National Forests. 

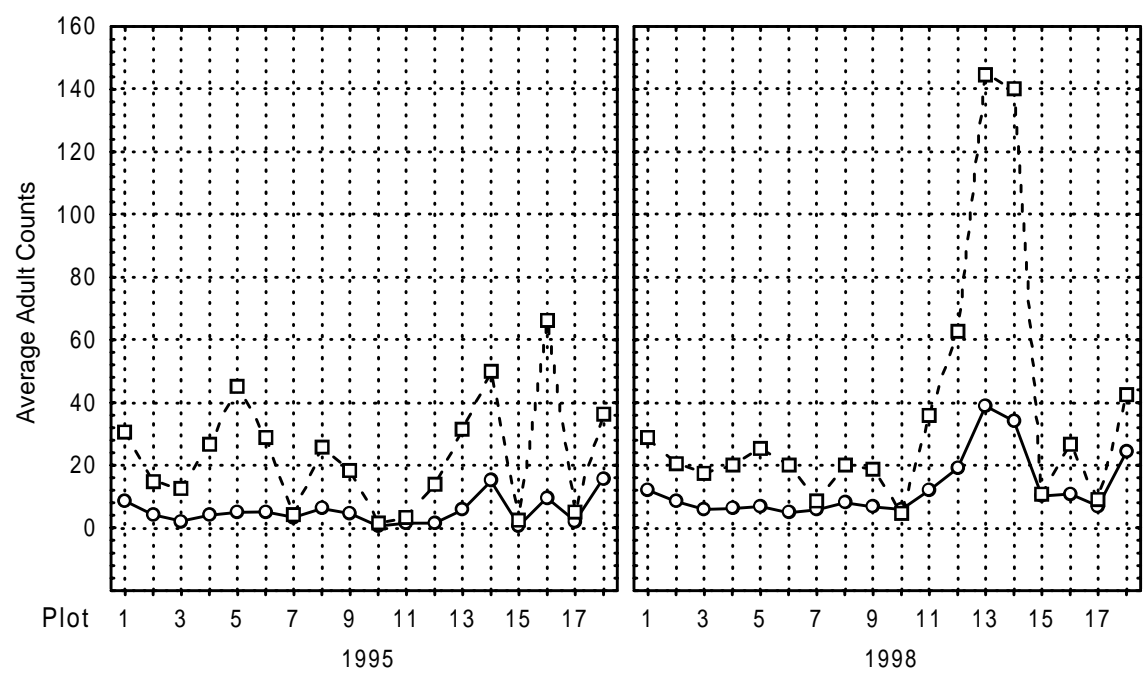

Reference:

(plots 2, 4, 8, 12, 14, 16)

$B t$ :

(plots $3,5,7,11,15,18$ )

Gypchek:

(plots 1, 6, 9, 10, 13, 17)

- - Females

--.- Males

Female and Male Wing Lengths (1995 \& 1998)

Acronicta ovata
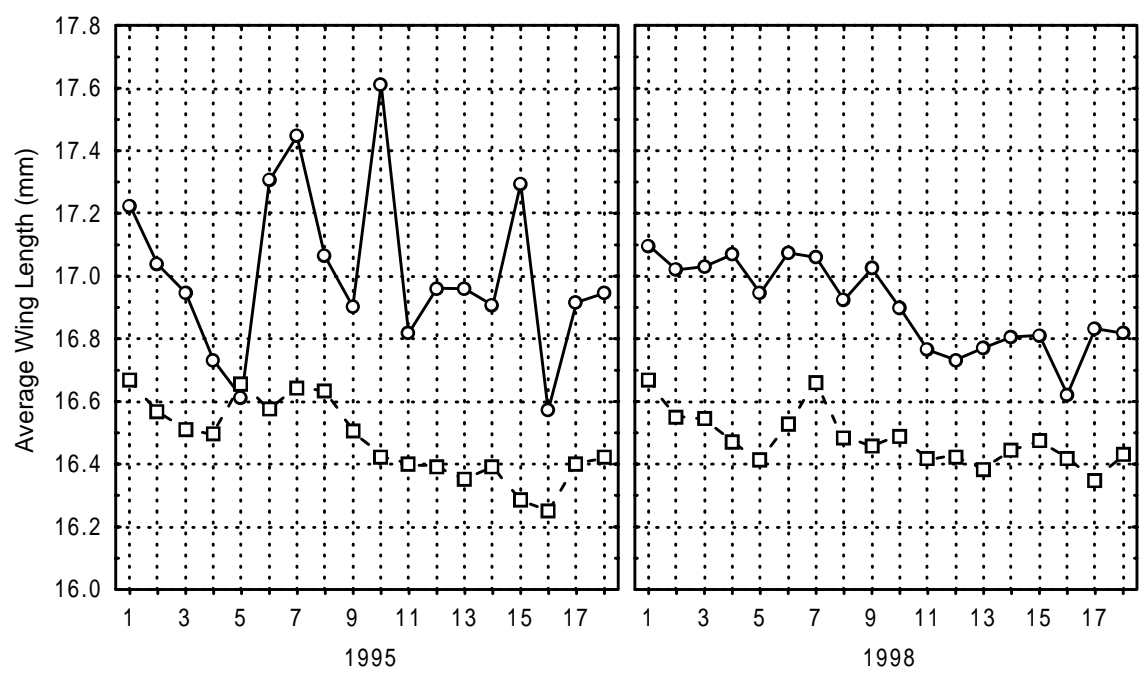

Reference:

(plots 2, 4, 8, 12, 14, 16)

$B t$ :

(plots $3,5,7,11,15,18$ )

Gypchek:

(plots 1, 6, 9, 10,13,17)

-o- Females

- - - Males

Figure 69. Female and male adult counts and wing lengths (1995 \& 1998). Graphs illustrate average plot counts and wing lengths of female and male Acronicta ovata collected during the 1995 pretreatment and 1998 treatment years from the George Washington (plots 1-9) and Monongahela (plots 10-18) National Forests. 


\section{Discussion:}

\section{A. Summary of Lepidopteran Statistical Trends \\ Pretreatment Years versus Treatment Years}

Total larval counts were significantly higher during treatment years than during pretreatment years. Significant increases were indicated for total noctuid counts, Polia latex, Morrisonia confusa, for the geometrid Besma endropiaria, and for the notodontid Heterocampa guttivitta. Significant decreases in larval counts during treatment years were seen in the geometrids Besma quercivoraria and Melanolophia canadaria, for the notodontid Nadata gibbosa, and for the arctiids Hypoprepia fucosa and Halysidota tesselaris.

Total adult counts were significantly higher during treatment years than during pretreatment years. Significant increases were seen in total noctuid counts, Polia latex, Morrisonia confusa, Allotria elonympha, Baileya opthalmica, Acronicta ovata, for the geometrids Lambdina fervidaria, Campaea perlata, Tetracis cachexiata, Besma endropiaria, for the notodontid Heterocampa guttivitta, and for the arctiid Halysidota tesselaris. When significant differences were indicated by both larvae and adults, numbers of both stages increased with the exception of $H$. tesselaris, which showed a lower number of larvae and a higher number of adults collected during treatment years. Significant decreases in adult counts during treatment years were demonstrated in the geometrids Hypagyrtis unipunctata and Besma quercivoraria, for the notodontid Nadata gibbosa, and for the arctiid Hypoprepia fucosa.

\section{Forests:}

Larvae of 6 species showed significantly greater counts in the MON than in the GW. Adults of 9 species showed significantly greater counts in the MON than in the GW. All 19 species combined and the geometrids combined showed greater total counts in the MON compared to the GW, as well. Allotria elonympha was the only moth species to show significantly greater adult numbers in the GW. 


\section{Year x Forest (Sex)}

Larvae of 4 species showed similar counts in the GW and MON during pretreatment years, but significantly greater counts in the MON during treatment years. All 19 species combined and the geometrids combined showed similar trends. Larvae of 2 species showed the opposite trend. Itame pustularia was an exception that showed significantly more larvae in the GW during pretreatment years and significantly more larvae in the MON during treatment years. When SEX was added to the ANOVA model for 1995 and 1998 data, the differences observed were attributed to fluctuations in male catches. Females remained comparatively constant in number. In all but one case, significantly more adult males were collected than adult females. Male and female count differences were not significant for Morrisonia confusa.

\section{Year x Treatment}

Direct treatment effects were not indicated by statistical analyses. However, the Year x Treatment interaction for larvae of 3 species (Lambdina fervidaria, Heterocampa guttivitta, and Achatia distincta) showed significantly lower counts in Bt plots when compared to Ref and/or GC plots during treatment years. Although by chance approximately $5 \%$ of the statistical analyses conducted might be expected to be significant, it should be noted that in each case, the lower counts were indicated for $B t$ plots during treatment years relative to the other categories (see Figures 21, 44, and 55; YEAR x TREATMENT interactions). It should be noted that although fewer larvae were collected from $B t$ plots during treatment years when compared to $B t$ plots during pretreatment years, the differences were not significant. The adults of Hypagyrtis unipunctata and Malacosoma disstria showed a similar trend (see Figures 17 and 41).

Counts in $B t$ plots during treatment years were significantly lower by comparison to $B t$ plots during pretreatment years, and also by comparison to GC plots during both pretreatment and treatment years. Male moths in $B t$ plots were reduced most drastically. 


\section{Wing Lengths}

Sublethal effects correlated with Bt or GC treated plots were not identified. However, several trends were observed. When sufficient numbers of adults of each sex were obtained for an ANOVA, wing length difference was found to be significant, indicating that females were larger than males. However, although too few females of Melanolophia canadaria were collected for an ANOVA, Student's t-tests indicated that males were larger than females in the MON, but not in the GW. The wing lengths of all 19 species combined, the geometrids combined, Melanolophia canadaria (only males included), Lambdina fervidaria, and Itame pustularia, showed increases from the 1995 pretreatment year to the 1998 treatment year. Wing lengths of three species (Hypagyrtis unipunctata (only males included), Morrisonia confusa, and Baileya opthalmica) showed significant decreases from 1995 to 1998 . The noctuids combined, Tetracis cachexiata, Halysidota tesselaris, Polia latex, Morrisonia confusa, and Acronicta ovata were significantly larger in the GW than the MON. Lambdina fervidaria and Heterocampa guttivitta were larger in the MON than the GW.

Statistical interactions indicated overall trends in wing length of the 19 species combined. The GW species were comprised of those with larger females than the MON species. Males of the MON species were larger than males of the GW species. These trends were also observed for the geometrids combined.

\section{Larval and Pupal Weights}

Average weights of Polia latex larvae collected from the GW were greater than the average weights of the larvae collected from the MON. Similarly, the average pupal weights from larvae collected from the GW and reared in the laboratory were heavier than those resulting from larvae collected from the MON. Comparison of the larvae of Besma endropiaria showed no significant difference. 


\section{B. Implications of Lepidopteran Trends}

\section{Fluctuations in Population Abundances}

In addressing yearly fluctuations and forest differences in lepidopteran populations, both biotic factors (i.e. disease, predation, and competition), as well as abiotic factors (i.e. physical and chemical characteristics) must be considered. The study was designed to reduce confounding variables such that potential treatment effects could be better isolated. To that end, forests of similar vegetation type were chosen and subjectively blocked accordingly. Likewise, sampling events took place simultaneously in each plot throughout the study. Still, soil differences (i.e. particulate size, composition, organic content, buffering capacity, moisture), seasonal variations (i.e. weather, winds, cloud cover), geological features (i.e. ridge aspect, elevation), pollutants (i.e. acid rain, mine drainage), land usage (i.e. logging, mining, agriculture), and various other components can affect insect populations, as well as the quality of the foliage on which herbivores feed. As the two forests were expected to exhibit some differences in several of these physical parameters, potential differences in insect populations were likewise expected.

The GW forest, located further south and east of the MON forest, is more xeric and the plots are an average $315 \mathrm{~m}$ lower in elevation in the GW than in the MON. Elevation, latitudinal, and longitudinal differences might be expected to produce a cooler, shorter growing season in the MON compared to the GW. The MON forest was shown to be significantly cooler than the GW forest during both pretreatment and treatment years. Larvae and adult data show in several instances that peak abundance was often delayed 1 to 2 weeks or more in the MON in comparison with the peak abundance in the GW. Still, although a cold spring may impact some early season larval species, it should be noted that much of the increase in larval numbers can be attributed to later season species which would not necessarily be adversely affected by the cool early season temperatures. Indeed, larvae of Acronicta ovata, extremely abundant in the Mon (especially during treatment years), are only seen later in the season. 
Seasonal differences were also noted in that average combined temperatures for the first six weeks of the two years preceding treatment applications in the MON were significantly cooler than the corresponding temperatures during treatment years. This was not the case in the GW. As pointed out by Connell (1983); Lawton and Strong (1981); Fitzgerald (1995), bad weather during early developmental instars can have a profound detrimental effect on lepidopteran larvae.

Foliage moisture content differences between forests (i.e. GW more xeric than MON) might also contribute at some level to fluctuations in lepidopteran abundances. As discussed by Bernays (1992), if given the choice, many insect herbivores chose wet leaves over dry leaves. If for nutritional reasons such a choice is adaptive (i.e. relative to survival rates or fecundity), a tougher, less succulent foliage might influence egg deposition by moth populations.

Although herbivory has been known to affect foliage quantity, competition may have had little to do with population fluctuations in this particular study due to the fact that foliage samples showed extremely low numbers of caterpillars, and other herbivorous insects, relative to the enormous number of intact leaves gathered from samples in both forests. This is apparently common in that plant foliage may often be superabundant and not resource limiting (Hairston et al. 1960). According to Schoener (1983), in such cases, abundances would likely be more influenced by predation than competition. In particular, lepidopteran population fluctuations may be caused by interactions with parasitoid populations (as reviewed by Berryman 1996). Unfortunately, a 4-year study utilizing two different forests (such as this study) would likely prove to be inadequate to determine complete oscillations of predator-prey cycles, which could be identified only after many years of intensive study. Likewise, effects of disease on population cycles are difficult to determine in a 4-year period.

Females were less abundant in catches than males. This is not surprising as males are typically attracted to calling females utilizing pheromones. Although females may be more sedentary in comparison to males, light traps may also be less attractive to (or less encountered by) females (Young 1997). At least 
some females of each of the species selected in this study were collected. Although numbers were low, they remained relatively constant in comparison to males. Interestingly, changes in sex ratios may be important in determining population dynamics in outbreak species (Mauffette and Jobin 1985).

Females of some species have been known to emerge slightly later than males; however, this was not readily determined in this study. Weekly sampling events may not have been frequent enough to separate emergence differences if these differences were only a few days apart. Likewise, for moth counts as a whole, weekly sampling events may have missed certain peaks in population densities if moths emerged, mated and died between sampling events. Indeed, in the case of Malacosoma disstria, females may emerge, breed, and die within a of matter twenty-four hours to five days (Fitzgerald 1995).

Another limitation in assessing population fluctuations and abundances stems from the fact that the sampling season for this study was limited to only 15 weeks beginning in early May and ending in midAugust. Many of the larvae and adults of the selected species are present before and/or after the sampling season. Early spring broods or late summer and fall broods may thus be inadequately sampled. Seasonal population shifts over the 4-year period could potentially be responsible for amplified or underestimated abundances when comparing catches to the actual forest populations.

\section{Potential $B t$ Lethal Effects}

Significantly lower numbers of larvae were noted in $B t$ plots during treatment years when compared to Ref and/or GC plots for three species in particular. Of these, Lambdina fervidaria and Achatia distincta larvae are both present during the early spring when treatments were applied, so exposure was likely. However, in the case of Achatia distincta, only 56 larvae were collected, which may potentially reduce the strength of any inferences implied by the statistical analyses. For L. fervidaria, however, 136 larvae were collected. Although Heterocampa guttivitta larvae were also in lower numbers in Bt plots, it is unlikely that $B t$ was responsible due to the fact that these larvae appear later in the season, after $B t$ is likely to have 
become ineffective as a lepidopteran toxin. The breakdown of $B t$ toxins proceeds rapidly, as reviewed by Reardon et al. (1994), due mostly to solar degradation. Within a matter of hours, or more conservatively, within a few days, $B t$ toxin levels are expected to be ineffective in reducing most lepidopteran larval populations. Subsequent bio-assay analysis, not addressed in this study, was conducted using gypsy moth larvae. Larvae were reared in the laboratory and fed Btk treated leaves collected from treatment plots 2 weeks after application. Treatment effects on the gypsy moth larvae were not identified.

In each of the cases previously mentioned, when pretreatment years were included in the analyses, variability was such that population depressions in $B t$ plots during treatment years were not statistically significant from pretreatment $B t$ plots. Interestingly, when the 19 selected species were combined and when all the non-target foliage lepidopteran larvae were combined, analyses of the data from the first 5 weeks of the sampling seasons showed a similar trend in the depression of larval counts in $B t$ plots during treatment years (see Figure 4). Other non-target studies have shown similar declines in macrolepidopteran species abundance and/or richness when comparing Bt treated areas to untreated areas (Miller 1990a,b; Lih 1994; Butler et al. 1995). In the present study, though the interaction was not significant, the YEAR, FOREST, and YEAR x FOREST effects were significant. Although more larvae were collected during treatment years overall, when compared to pretreatment years, the early season larvae collected during the first five weeks showed the reverse trend. Most of the decrease in early season numbers was attributed to a lower number of early season GW larvae collected during treatment years.

The only adult species showing significant population reductions in Bt plots compared to both GC and Ref categories during treatment years was Malacosoma disstria. This species has been known to be $B t$ sensitive (Fitzgerald 1995). The YEAR x TREATMENT interaction showed similar trends as the larvae mentioned above, but again pretreatment year variability obscured the effect. Unfortunately, only 39 larvae were collected and analysis could not be reasonably conducted. However, as these larvae are present in early spring, when the $B t$ was applied in this study, contact with the toxin was likely unavoidable. 
Potential Bt effects on Hypagyrtis unipunctata was also identified. Moth counts were significantly lower in Bt plots during treatment years than during pretreatment years. The difference is also significant when compared to GC plots. The Ref plots during pretreatment and treatment years, however, were not significantly different from Bt plots. Interestingly, sexed moths from 1995 and 1998 showed an interaction such that males in $B t$ plots showed the most drastic reduction in numbers (see Figure 19). It should be reiterated that an increased sensitivity in males of the spruce budworm has been previously identified by Pedersen et al. (1997). Although counts of the larvae showed no similar significant reductions, the potential for larvae to be exposed to $B t$ was likely for the early season brood. It should be noted that early season peaks in larval counts were not observed during treatment years, although early season peaks appeared during pretreatment years.

\section{Potential $B t$ Sublethal Effects}

Sublethal effects of $B t$ evidenced by wing length differences or in larval/pupal weight differences were not identified in this study. According to Pedersen et al. (1997), sublethal effects of $B t$ toxins may differ depending on the developmental stage of larvae at the time of exposure. Such differences may be evidenced by reduced pupal weights, delayed developmental rates, or decreased fecundity. Consequently, as applications of $B t$ in this study were based on optimal susceptibility of gypsy moth larvae, timing may have precluded sublethal toxic effects on some other larvae present at the time. Indeed, for a toxin to be most effective, it must be applied at the most susceptible developmental stage of the larvae (Reardon et al. 1994). Larvae of many of the selected species in this study are later season larvae which would have appeared long after the $B t$ treatments had lost effectiveness. Although numerous lepidopteran species show the ability to develop resistance to $B t$ toxins (Tabashnik 1994), it is unlikely that the larvae in this particular study have had such an opportunity. Local populations have not historically been exposed to the $B t$ toxins applied in this study. Interestingly, $B t$ toxins may also differ in effectiveness against some herbivorous insects 
depending on what host plant is sprayed (Harcourt et al.1996) or what Bt strain is used. This may possibly be due to interactions with the plants or the quantity of host material ingested by the insect.

Relative to sex differences in wing lengths, female wing lengths were typically found to be longer than male wing lengths within a species. As previously mentioned, larger female body size is common. Apparently larger, more sedentary females are able to concentrate their resources on egg production.

Yearly differences comparing wing lengths of moths indicated that in several cases, overall size of the moths showed an increase from the 1995 pretreatment year to the 1998 treatment year (see Tables 7 and 9). This occurred for the combined 19 species, combined geometrids, Melanolophia canadaria, Lambdina fervidaria, and for Itame pustularia. Larvae of these moths are typically found earlier in the season than most of the other species. The larvae of the three adult species showing decreases in size (Hypagyrtis unipunctata, Morrisonia confusa, and Bailey opthalmica) are typically found in greatest abundance later in the season (although as previously mentioned, H. unipunctata may also have an earlier peak).

Wing length differences between forests were also identified. For the combined noctuids, Tetracis cachexiata, Halysidota tesselaris, Polia latex, Morrisonia confusa, and Acronicta ovata, wing lengths were significantly longer in the GW than when compared to the MON. Only Lambdina fervidaria and Heterocampa guttivitta males showed the reverse trend. A significant size difference was also identified for the larvae and resulting pupae of Polia latex. Again, the size increase was for the GW larvae/pupae. Many lepidopteran species, including tent caterpillars (Fitzgerald 1995), show a direct correlation with the number of eggs produced by a female to her mass. Similarly, Pedersen et al. (1997) demonstrated in the spruce budworm that a reduction in size due to delayed development caused by $B t$ toxins correlated with reduced fecundity. Still, polyphagous species of large size may simply have large eggs. The adaptive value of being large could, however, have physiological implications that cause the insect to be less sensitive to environmental stresses (Wasserman and Mitter 1978). 
Larger sizes could correlate with higher temperatures and longer growing season of the GW. Indeed, considering the specimens collected from northern and southern regions within the range of a species, dwarfs are typically evidenced in the northern climates or in second broods that develop late in the season (Young 1997). Cooler temperatures directly slow the developmental rate of larvae that ultimately result in smaller individuals (Fitzgerald 1995; Lindroth et al. 1997). Interestingly, as pointed out by Strong et al. (1984), cooler temperatures may slow larval development, but not necessarily foliage development. The result may be stunting due to the young larvae that miss their "window of opportunity" to feed on freshly developing leaves. Old leaves may thus result in small larvae and pupae (and ultimately adults). In the gypsy moth, it has been noted that population abundance may be inversely related to size. This is likely due to a decrease in foliage quality or a reduction in preferred host availability (Lance et al.1986 and 1991).

Indeed foliage differences, even within the same species of plant, may contribute to size differences in insect herbivores. As discussed by Strong et al. (1984), tougher foliage (such as might be due to dry conditions, or older leaves resulting from an extended growing season) might be more easily consumed by larger caterpillars equipped with larger mandibles. If this is true, selective pressures might drive local populations in such an area to be more successful if minor gene variations provide for an increase in size. Decreases in moth size seasonally, as demonstrated for the combined 19 species, combined geometrids, and combined noctuids, might likewise reflect a decrease in foliage quality. Soil and nutrient differences (Bernays 1992; Young, 1997), nitrogen content (Lindroth et al. 1997), and even sun and shade (Strong et al. 1984) may further affect a potential host plant, making it more or less nutritional to an insect herbivore. Interestingly, as discussed by Lindroth et al. (1997), nitrogen content and temperature may show interactive effects relative to larval development and survival.

The seemingly odd appearance of an overall SEX x FOREST interaction (with regard to size), when all the species are combined, becomes more clear when consideration is given to the fact that this interaction was also significant for the geometrids combined. In two instances (Melanolophia canadaria and 
Hypagyrtis unipunctata), females were not included in the ANOVA due to insufficient numbers. However, Student's t-tests indicated that females of these two species were significantly smaller in the MON in comparison to the GW; males were larger. For M. canadaria, an ANOVA using only SEX and FOREST indicated that the difference was due to small MON females, otherwise males and females were of comparable wing lengths. Males were shown to be larger than females if the GW data were not included in the 4-way ANOVA. For H. unipunctata, males on both forests were comparatively smaller than females in the GW. Females in the MON, however were approximately the same size as the males. Smaller female wing lengths were also observed in the MON for Besma quercivoraria and Besma endropiaria, although the differences were not significant. The differences, however, would be additive when combined analyses are conducted. It is well documented that cooler temperatures are often correlated with a reduction in female wing length in many of the geometrids (i.e. Forbes 1948). Although they may sacrifice some capacity for flight, apparently the investment into egg production and an increased abdominal capacity offsets such a seeming "disadvantage". It should be noted that Lambdina fervidaria, one of the two species to show a decrease in wing length when comparing MON to GW moths, is an early season geometrid as well.

\section{Possible Difficulties}

In this study, one problem in the attempt to identify detrimental effects due to treatments occurred because light trap catches tell nothing of host plant selection by larvae. Individual sizes, developmental rates, and overall reproductive success can be highly host plant specific (Barbosa and Capinera 1977; Capinera and Barbosa 1977; Strong et al. 1984; Miller and Hanson 1989; Hamilton and Lechowicz 1991). Even within local populations, differences in host plant choice may be identified, ultimately depending on which plants females choose for egg deposition. Although larger species of polyphagous moths appear less host specific, some noctuids show regional differences (Wasserman and Mitter 1978; Bernays 1992). 
Gross differences in catches between forests are markedly easier to link to foliage or physical parameters than are individual plot differences.

Still, the capacity for the adults to fly, sometimes very long distances, makes the correlation between light trap locations and the locations where the larvae had fed much weaker. As discussed by Fitzgerald (1995), female tent caterpillars may fly two or three miles. In one case, moths found in a new location were suspected to have originated from a location 300 miles distant. Winds of a cold front were thought to have carried the moths to their new location in a period of only 12 hours.

Consequently, when considering data collected utilizing light trap catches, potential treatment effects would be conservative. Similarly, if light trap catches are selective relative to adult size (i.e. larger moths more frequently captured), potential sublethal effects might also be conservative. Larval data, conversely, as foliage samples are collected from the same tree species in all plots, would be expected to be more effective in showing potential treatment effects among groups. Still, yearly variability may obscure treatment effects unless such effects are catastrophic in nature.

Weather variability was also difficult to assess. According to Williams and Liebhold (1995), population oscillations may be linked to weather factors. In that study, it was determined that environmental factors may act to synchronize gypsy moth populations across broad regions.

Relative humidity might have been a better parameter to consider rather than utilizing rain gauges. Likewise, although maximum-minimum thermometers may yield some limited data relative to variability among plots, temperatures during the off-season months were not determined. Therefore potential effects due to fall or winter variability were not analyzed. Similarly, long-term seasonal variability could not be determined in a 4-year study of this nature. Both biotic and abiotic factors acting to stabilizing or destabilize moth populations would be nearly impossible to determine without additional information. Perhaps by continuing to monitor yearly variability in moth populations and attempting to further quantify weather and seasonal characteristics, stronger conclusions could be drawn. 


\section{Literature Cited:}

Andreadis, T.G. and R.M. Weseloh. 1990. Discovery of Entomophaga maimaiga in North American gypsy moth, Lymantria dispar. Proceedings of the National Academy of Science. 87:2461-2465.

Barber, K.N., W.J. Kaupp, and S.B. Holmes. 1993. Specificity testing of the nuclear polyhedrosis virus of the gypsy moth, Lymantria dispar (L.) (Lepidoptera: Lymantriidae). Canadian Entomologist. 125:10551066.

Bernays, E.A. 1992. Insect-Plant Interactions Vol. IV. CRC Press. 226pp.

Bernays, E.A., and R.F. Chapman. 1995. Host-Plant Selection by Phytophagous Insects. Chapman \& Hall. New York, New York. 312pp.

Berryman, A.A. 1996. What causes population cycles of forest Lepidoptera? Trends in Ecology and Evolution. 11:28-32.

Barbosa, P. and J.L. Capinera. 1977. The influence of food on developmental characteristics of the gypsy moth, Lymantria dispar (L.). Canadian Journal of Zoology. 55:1424-1429.

Butler, L. 1992. The community of macrolepidopterous larvae at Coopers Rock State Forest, West Virginia: a baseline study. Canadian Entomologist. 124:1149-1156.

Butler, L., and V. Kondo. 1991. Macrolepidopterous moths collected by blacklight trap at Coopers Rock State Forest, West Virginia: a baseline study. West Virginia Agriculture and Forestry Experiment Station Bulletin. 705. 25pp.

Butler, L., and V. Kondo. 1993. Impact of Dimilin on non-target Lepidoptera: Results of an operational gypsy moth suppression program at Cooper's Rock State Forest, West Virginia. West Virginia Experiment Station Bulletin. 701. 21pp.

Butler, L., G.A. Chrislip, V.A. Kondo. 1995a. Canopy arthropods at Fernow Experimental Forest in West Virginia's Allegheny Mountain Section: II. Macrolepidopterous larvae on foliage and under burlap bands. West Virginia Agriculture and Forestry Experiment Station Bulletin. 713. 16pp.

Butler, L., C. Zivkovich, and B.E. Sample. 1995b. Richness and abundance of arthropods in the oak canopy of West Virginia's Eastern Ridge and Valley Section during a study of impacts of Bacillus thuringiensis with emphasis on macrolepidoptera larvae. West Virginia Agricultural Experiment Station Bulletin. 711.17pp.

Butler, L., G.A. Chrislip, V.A. Kondo, and E.C. Townsend. 1997a. Effect of Diflubenzuron on nontarget canopy arthropods in closed, deciduous watersheds in a central Appalachian forest. Journal of Economic Entomology. 90:784-794.

Butler, L. V. Kondo, and D. Blue. 1997b. Effects of Tebufenozide (RH-5992) for Gypsy moth (Lepidoptera: Lymantriidae) suppression on nontarget canopy arthropods. Environmental Entomology. 26:1010-1015. 
Cameron, E.A. and R.M. Reeves. 1990. Carabidae (Coleoptera) associated with gypsy moth, (Lepidoptera; Lymantriidae) populations subjected to Bacillus thuringiensis Berliner treatments in Pennsylvania.

Canadian Entomologist. 122:121-129.

Capinera, J.L. and P. Barbosa. 1977. Influence of natural diets and larval density on gypsy moth, Lymantria dispar (Lepiodoptera: Orgyiidae), egg mass characteristics. The Canadian Entomologist. 109:1313-1318.

Casagrande, R.A., P.A. Logan, and W.E. Wallner. 1987. Phenological model for gypsy moth, Lymantria dispar L. (Lepidoptera: Lymantriidae), larvae and pupae. Environmental Entomology. 16:556-562.

Cloutier, C. and C. Jean. 1998. Synergism between natural enemies and biopesticides: a test case using the stinkbug Perillus bioculatus (Hemiptera: Pentatomidae) and Bacillus thuringiensis tenebrionis against the Colorado potato beetle (Coleoptera: Chrysomelidae). Journal of Economic Entomology. 91:1096-1108.

Connell, J.H. 1983. On the prevalence and relative importance of inter-specific competition: Evidence from field experiments. The American Naturalist. 122:661-697.

Cooper, R.J. and Whitmore. 1990. Arthropod sampling methods in ornithology. Studies in Avian Biology. 13:29-37.

Covell, C. Jr., 1984. A Field Guide to Moths Eastern North America. Houghton Mifflin Company. Boston, MA. 496pp.

Crossley, D.A.Jr., J.T. Callahan, C.S. Gist, J.R. Maudsley, and J.B. Waide. 1976. Compartmentalization of arthropod communities in forest canopies at Coweeta. The Journal of the Georgia Entomological Society. 11:44-49.

Crumb, S.E. 1955. The Larvae of the Phalaenidae. United States Department of Agriculture, Washington D.C. Technical Bulletin No. 1135. 356pp.

Denno, R.F., M.S. McClure, and J.R. Ott. 1995. Inter-specific interactions in phytophagous insects:

Competition reexamined and resurrected. Annual Review of Entomology. 40:297-331.

Dubois, N.R. 1998. Unpublished data on file at USDA Forest Service Laboratory, Hamden CT.

Dubois, N.R. and D.H. Dean. 1995. Synergism between CryIA insecticidal crystal proteins and spores of Bacillus thuringiensis, other bacterial spores, and vegetative cells against Lymantria dispar (Lepidoptera: Lymantriidae) larva. Environmental Entomology. 24:1741-1747.

Fitzgerald, T.D. 1995. The Tent Caterpillars. Cornell University Press, Ithaca, New York. 303pp.

Forbes, W.T.M. 1948. Lepidoptera of New York and Neighboring States. Geometridae, Sphingidae, Notodontidae, Lymantriidae. Part II. Memoir 274. Cornell Agricultural Experiment Station. Ithaca, New York. 263pp.

Forbes, W.T.M. 1954. Lepidoptera of New York and Neighboring States. Part III. Noctuidae. Memoir 329. Cornell Agricultural Experiment Station. Ithaca, New York. 433pp. 
Forbes, W.T.M. 1960. Lepidoptera of New York and Neighboring States. Agaristidae through Nypmphalidae including Butterflies. Part IV. Memoir 371. Cornell University Agricultural Experiment Station, New York State College of Agriculture. Ithaca, New York. 188pp.

Franclemont, J.G. 1973. The Moths of America North of Mexico. Fascicle 20.1. E.W. Classey Limited and R.B.D. Publications, Inc. London, England. 86pp.

Hairston, N.G., F.I. Smith, and L.B. Slobodkin. 1960. Community structure, population control and competition. The American Naturalist. 94:421-425.

Hajek, A.E., R.I. Carruthers, and R.S. Soper. 1990a. Temperature and moisture relations of sporulation and germination by Entomophaga maimaiga (Zygomycetes: Entomophthoraceae), a fungal pathogen of Lymantria dispar (Lepidoptera: Lymantriidae). Environmental Entomology. 19:85-90.

Hajek, A.E., R.A. Humber, J.S. Elkinton, B. May, S.R.A Walsh, and J.C. Silver. 1990b. Allozyme and restriction fragment length polymorphism analyses confirm Entomophaga maimaiga responsible for 1989 epizootics in North American gypsy moth populations. Proceedings of the National Academy of Science. 87:6979-6982.

Hajek, A.E., R.A. Humber, and J.S. Elkinton. 1995. Mysterious origin of Entomophaga maimaiga in North America. American Entomologist. 41:31-42.

Hajek, A.E. and R.S. Soper. 1992. Temporal Dynamics of Entomophaga maimaiga after death of gypsy moth (Lepidoptera: Lymantriidae) larval Hosts. Environmental Entomology. 21:129-135.

Hamiliton, D.J. and M.J. Lechowicz. 1991. Host effects on the development and fecundity of gypsy moth, Lymantria dispar L., reared under field conditions. Canadian Journal of Zoology. 69:2217-2223.

Harcourt, R.L., D. Llewellyn, R. Morton, E.S. Dennis, and W.J. Peacock. 1996. Effectiveness of purified Bacillus thuringiensis Berliner insecticidal proteins in controlling 3 insect pest of Australian eucalypt plantations. Journal of Economic Entomology. 89:1392-1398.

Holloway, J.D. 1984. Moths as indicator organisms for categorizing rain-forest and monitoring changes and regeneration processes. Tropical Rain Forest: The Leeds Symposium. 235-242pp.

Lance, D.R., J.S. Elkinton, and C.P. Schwalbe. 1986. Feeding rhythms of gypsy moth larvae: Effect of food quality during outbreaks. Ecology. 67:1650-1654.

Lance, D.R., J.S. Elkinton, and C.P. Schwalbe. 1991. Responses of gypsy moth larvae (Lepidoptera: Lymantriidae) to foliage of oaks from naturally infested sites on Cape Cod, Massachusetts. Journal of Entomological Science. 26:214-222.

Lawton, J.H., and D.R. Strong, Jr. 1981. Community patterns and competition in folivorous insects. American Naturalist. 118:317-338.

Liebhold, A. and M. McManus. 1999. The evolving use of insecticides in gypsy moth management. Journal of Forestry. 97:20-23. 
Lih, M.P., F.M. Stephen, K.G. Smith, L.R. Nagy, and G.W. Wallis. 1994. Effects of gypsy moth eradication program on nontarget forest canopy insects and their bird predators. Proceedings 1994 Annual Gypsy Moth Review. 193-201.

Lindroth, R.L., K.A. Klein, J.D.C. Hemming, and A.M. Feuker. 1997. Variation in temperature and dietary nitrogen affect performance of the gypsy moth (Lymantria dispar L.). Physiological Entomology. 22:55-64.

Mauffette, Y. and L. Jobin. 1985. Effects of density on the proportion of male and female pupae in gypsymoth populations. The Canadian Entomologist. 117:535-539.

Miller, J.C. 1990a. Field assessment of the effects of a microbial pest control agent on non-target Lepidoptera. American Entomologist. 36:135-139.

Miller, J.C. 1990b. Effects of a microbial insecticide, Bacillus thuringiensis kurstaki on non-target Lepidoptera in a spruce budworm-infested forest. J. Res. Lepidop. 29:267-276.

Miller J.C. and P.E. Hanson. 1989. Laboratory studies on development of gypsy moth, Lymantria dispar (L.) (Lepidoptera: Lymantriidae), larvae on foliage of gymnosperms. The Canadian Entomolgist. 121:425429.

Moran, V.C. and T.R.E. Southwood. 1982. The guild composition of arthropod communities in trees. The Journal of Animal Ecology. 51:289-306.

Overgaard Nielsen, B., and A. Ejlersen. 1977. The distribution pattern of herbivory in a beech canopy. Ecological Entomology. 2:293-299.

Pedersen, A., J. Dedes, D. Gauthier, and K. van Frankenhuyzen. 1997. Sublethal effects of Bacillus thuringiensis on the spruce budworm, Choristoneura fumiferana. Entomologia Experimentalis et Applicata. 83:253-262.

Pielou, D.P. (editor). 1972. Guide to the Geometridae of Canada (Lepidoptera) II, Subfamily Ennominae. I. W.C. McGuffin. Department of the Environment Canadian Forestry Service. Ottawa, Canada. Memoirs of the Entomological Society of Canada. No. 86pp.

Podgwaite, J.D., R.C. Reardon, G.S. Walton, L. Venables, and D.M. Kolodny-Hirsch. 1992. Effects of aerially applied Gypchek on gypsy moth (Lepidoptera: Lymantriidae) populations in Maryland woodlots. Journal of Economic Entomology. 85:1136-1139.

Reardon, R., N. Dubois, and W. McLane. 1994. Bacillus thuringiensis for managing gypsy moth: a review. Nation Center of Forest Health Management: Technology Transfer. USDA Forest Service. FHM-NC-0194. 32pp.

Roth, S., C. Knorr, and R.L. Lindroth. 1997. Dietary phenolics affects performance of gypsy moth (Lepidoptera: Lymantriidae) and its parasitoid Cotesia melanoscela (Hymenoptera: Braconidae). Physiological and Chemical Ecology. 26:668-671.

Sample, B.E., L. Butler, C. Zivkovich, and R.C. Whitmore. 1993. Evaluation of Bacillus thuringiensis and defoliation effects on native Lepidoptera. USDA Forest Service, AIPM Technology Transfer. NA-TP-1093. 12pp. 
Sample, B.E., L. Butler, C. Zivkovich, R. Whitmore, and R. Reardon. 1996. Effects of Bacillus

thuringiensis and defoliation by the gypsy moth on native arthropods. Canadian Entomologist. 128:573-592.

Schoener, T.W. 1983. Field experiment on inter-specific competition. The American Naturalist. 122:240285.

Strong, D.R., J.H. Lawton, S.R. Southwood. 1984. Insects on Plants; Community Patterns and Mechanisms. Harvard University Press, Cambridge, Massachusetts. 313pp.

Tabashnik, B.E. 1994. Evolution of resistance to Bacillus thuringiensis. Annual Review of Entomology. 39:47-79.

Tietz, H.M. 1972. Index to Life Histories North American Macrolepidoptera I \& II. Published by A.C. Allyn for the Allyn Museum of Entomology. Sarasota, Florida. 1041pp.

Wagner, D.L., V. Giles, R.C. Reardon, and M.L. McManus. 1997. Caterpillars of Eastern Forests. Forest Health Technology Enterprise Team-Morgantown, West Virginia. United States Department of Agriculture. FHTET-96-34. 113pp.

Wagner, D.L., J.W. Peacock, J.L. Carter, and S.E. Talley. 1996. Field assessment of Bacillus thuringiensis on nontarget Lepidoptera. Environmental Entomology. 25:1444-1454.

Wasserman, S.S. and C. Mitter. 1978. The relationship of body size to breadth of diet in some Lepidoptera. Ecological Entomology. 3:155-160.

Weseloh, R.M. 1984. Effects of the feeding inhibitor Plictran and low Bacillus thuringiensis Berliner doses on Lymantria dispar (L.) (Lepidoptera: Lymantriidae): Implications for Cotesia melanoscelus (Ratzeburg) (Hymenoptera: Braconidae).

Wie, S.I., R.E. Andrewes, Jr., B.D. Hammock, R.M. Faust, and L.A. Bulla, Jr. 1982. Enzyme-linked immunosorbent assays for detection and quantitation of the entomocidal parasporal crystalline protein of Bacillus thuringiensis subspp. kurstaki and israelensis. Applied and Environmental Microbiology. 43:891894.

Williams, W. and A.M. Liebhold. 1995. Influence of weather on the synchrony of gypsy moth (Lepidoptera: Lymantriidae) outbreaks in New England. Environmental Entomology. 24:987- .

Wood, P.S., and L. Butler. 1989. Biology and immature stages of Polia latex (Guenee) (Noctuidae). Journal of the Lepidopterists' Society. 43:299-304.

Wood, P.S., and L. Butler. 1991. Biology of Morrisonia confusa (Noctuidae). Journal of the Lepidopterists' Society. 45:197-203.

Young, M. 1997. The Natural History of Moths. University Press. Cambridge, England. 271pp. 


\section{Appendix I. Distribution, seasonality, and host plants of selected lepidopteran species.}

The geographical information for the following lepidopteran species, as well as adult appearance dates were obtained primarily from Forbes (1948, 1954, and 1960) and Covell (1984). Additional information relative to the larvae in West Virginia were obtained from Wood and Butler (1989), Wood and Butler (1991), Butler (1992), Butler et al. (1995a), Butler et al. (1995b), and from Wagner et al. (1997). Host plant information was summarized from the sources mentioned, from Crumb (1955), and Tietz (1972). The following species are presented in the order addressed in this study.

\section{Family Geometridae:}

Melanolophia canadaria (Gn.). Canadian Melanolophia. Larvae of this genus present during July from early June moths. Pupa overwinters. M. canadaria adults are present from March to September (second brood after July). Found commonly north to Quebec and Manitoba, south to New Jersey and Pennsylvania. Darker races appear south of Washington DC. West Virginia larvae collected from early May to early October, (peak mid June). Larvae found on several tree species including birch, cherry, elm, maple, oak, and pine.

Hypagyrtis unipunctata (Hbn.). One Spotted Variant. Found commonly north to Quebec; Sharon; New York; Sturgis; Michigan; Tyngsboro; Massachusetts. Morphologically variable. Adults present AprilSeptember. Found in Southern States north to North Carolina, west to Texas and Oklahoma. West Virginia larvae collected from late May to early October (peak mid June). Two-three broods in North. Larvae overwinter. Larvae found on several tree species including alder, birch, cherry, elm, fir, hickory, maple, oak, pine, and willow.

Lambdina fervidaria (Hbn.). Curved-lined Looper Moth. Found commonly north to Nova Scotia and Quebec, New Jersey, south to North Carolina, west to Wisconsin. Spring larvae found; mature larvae in September. Adults present April to August. Two broods. Larvae found on several tree species including ash, birch, beech, cherry, oak, and spruce.

Campaea perlata (Gn.). Fringed Looper, Pale Beauty. Adults present June and July, dwarfed in September. Late summer brood small. Part of pupae from first brood overwinter. Second brood partial in North. Found north to Newfoundland and Nova Scotia, south to North Carolina, west to Arizona and Yukon. West Virginia larvae collected from mid-to late June to mid-October (peak mid July). Two broods in North. Larvae found on various plant species including alder, birch, cherry, elm, fir, linden, maple, oak, poplar, and willow.

Tetracis cachexiata (Gn.). White Slant Line. Adults present April to June. Found north to Nova Scotia; Gaspe; Quebec; south to North Carolina, west to British Columbia. West Virginia larvae collected from late May to early October (peak late June). Two broods in North. Larvae found on various host plants including general shrubs, ash, basswood, birch, cherry, elm, iris, hemlock, maple, oak, pine, sheep laurel, sweet fern, and willow. 
Itame pustularia (Gn.). Lesser Maple Spanworm Moth. Adults present May to early September. Females of some may exhibit reduction in wing length. Larvae found particularly on maple and may be injurious. Found north to Nova Scotia; Lake St. Francis; Quebec; south to Florida, west to Wisconsin and Iowa. Eggs overwinter (Pielou, 1972). West Virginia larvae present from early May to early August. Larvae have also been found on birch, cherry, and oak, as well as maple.

Besma quercivoraria (Gn.). Oak Besma. Adults present April through September. Found north to Nova Scotia and Quebec, south to Florida, west to Nebraska and Texas. West Virginia larvae present late May to mid- late September (peak late May). Two broods. Larvae collected from cherry, elm, oak, poplar, white spruce, and willow.

Besma endropiaria (Gn.). Straw Besma. Adults present May to June, larvae follow. Pupae overwinter. Found north to Quebec, south to Maryland, west to Manitoba and Iowa. West Virginia larvae collected early July through early October (peak late July). One brood. Larvae on birch, cherry, maple, and oak.

\section{Family Lasiocampidae:}

Malacosoma disstria (Hbn.). Forest Tent Caterpillar Moth. Adults present April through September. Common throughout North America except Nevada and Arizona. No tents, but may be damaging. Larvae overwinter in egg shells, eclosing in early spring (Franclemont 1973). West Virginia larva collected from late May to mid June (peak late May). One brood. Larvae collected from numerous shrubs and trees including aspen, birch, cherry, cottonwood, gum, hickory, maple, and oak.

\section{Family Notodontidae:}

Heterocampa guttivitta (Wlk.). Saddled Prominent, Maple Prominent. Adults April to October. One brood. Larvae found in July and August. Found north to Nova Scotia and Quebec, west to Colorado and Manitoba, south to Florida. Can be pestiferous in North. Larvae in West Virginia from mid-June to midlate August (peak mid July). Two broods. Larvae on several tree species including apple, beech, birch, cherry, elm, hazel, hickory, maple, oak, poplar, and sumac.

Nadata gibbosa (J.E. Smith). White Dotted Prominent. Adults present June and July, a partial second brood in the fall. Found north to Nova Scotia and Quebec, south to Florida, west to California. West Virginia larvae found mid July through mid September (peak early August). Two broods in South. Larvae on several tree species including alder, beech, birch, cherry, maple, oak, plum and willow.

\section{Family Arctiidae:}

Hypoprepia fucosa (Hbn.). Painted Lichen Moth. Most of this genus overwinter as larvae, some as pupae. Present May to September. Found from Canada (not Eastern) to Florida, west to Minnesota and Manitoba. Larvae feed on mosses and lichens.

Halysidota tesselaris (J.E. Smith). Banded Tussock Moth, Pale Tussock Moth. Pupae overwinter under stones. Adults present early July and feed at flowers at dusk. Found north to Quebec and Minnesota, south to Florida (not south Florida or south Texas). West Virginia larvae collected late July to mid-October (peak mid August). One or two broods. Larvae on numerous plant species including alder, ash, birch, cherry, elm, hazelnut, hickory, maple, oak, poplar, sycamore, walnut, and willow. 


\section{Family Noctuidae:}

Achatia distincta (Hbn.). Distinct Quaker. Adults present late March to May. Found north to Quebec, south to Georgia, west to Texas and Colorado. West Virginia larvae found late May to mid-July (peak late May). One brood. Larval host plants include birch, flowering crabapple grape, linden, maple, oak, and walnut.

Polia latex (Gn.). Fluid Arches. Adults present late May and June (late May to late August in West Virginia; peaks June and July). One brood. Found primarily in Northeastern United States and adjacent Canada. West Virginia larvae found late May through mid- late September. Larvae are generalists on broad leaf deciduous trees such as beech, birch, cherry, elm, magnolia, maple, oak, and sassafras.

Morrisonia confusa (Hbn.). Confused Woodgrain. Adults present late April and May (mid-May to early June in West Virginia; peak late May). Found north to Canada and Maine, south to Florida, west to Texas and California, except Northwest. West Virginia larvae collected late May through mid- late September. One brood. Larvae on numerous shrubs and broad leaf trees including apple, basswood, birch, blueberry, cherry, hickory, maple, oak, pine, and willow.

Allotria elonympha (Hbn.). False Underwing. Adults present March to September. Found north to Canada and southern Maine, south to Florida, west to Tennessee. Host plants include black gum, sour gum, hickory, and walnut.

Bailey opthalmica (Gn.). Eyed Baileya. Adults late May through July. Found north to Nova Scotia and Quebec, west to Wisconsin, south to Alabama. Larval host plants include alder, beech, hickory, maple, oak, and ironwood.

Acronicta ovata (Grt.). Ovate Dagger Moth. Adults April through August. Found north to Nova Scotia and Manitoba, though may be "runty". Found south to Tennessee, west to Colorado and Texas. West Virginia larvae collected mid-July through early October (peak mid August). May be two or more broods. Larvae common on beech, birch, chestnut, and oak. 


\section{CURRICULUM VITAE}

Kenneth E. Rastall

Rd 2 Box 86B

Proctor, West Virginia 26055

\section{Education:}

-Ph D. Candidate, West Virginia University, College of Agriculture and Forestry, Division of Plant \& Soils Sciences--Entomology, Morgantown, West Virginia.

-Master of Arts Teaching -Biology (1987) -Central Michigan University, Mt. Pleasant, Michigan. Michigan Secondary Teaching Certification (biology/mathematics).

-Bachelor of Science-Biology, Mathematics minor (1984) -Lake Superior State University, Sault Ste. Marie, Michigan.

\section{Current Activities:}

West Virginia University (begun Fall 1995)

-Ph D. Candidate - Division of Plant \& Soil Sciences--Entomology Dissertation Title: Potential lethal and sublethal effects of gypsy moth biological treatments on non-target lepidopterans in two Appalachian forests.

\section{Wheeling Jesuit University (WJU)}

-Academic Associate- Instructor/Laboratory Coordinator (August 1992-Present) General Biology Laboratories/Human Anatomy Laboratories

-Mentoring Students:

Current responsibilities include assisting students in designing, conducting, and writing scientific papers on original research projects which they must ultimately present to faculty and peers (required for biology majors).

-Committee Membership:

Health Pre-professional Advisory Committee (1993-1998, Chair Fall 1997-Spring 1998).

-Student Advisor:

Advising students relative to course-work and career development. 
-Seminar Instructor:

"Explore Your Mind: Bio-Diversity- What Water Can Tell Us About the Environment". A two hour laboratory and field study introducing regional high school students to river-system ecological concepts.

\section{Present Professional Organization Memberships:}

-Entomological Society of America

-West Virginia Entomological Society

\section{Past Activities \& Research:}

\section{Wheeling Jesuit University}

-Wheeling Creek Project (1993-1995) - Dr. Ben Stout, WJU, coordinator

This study involved the collection and analysis of physical, chemical, and benthological macroinvertebrate data at more than 110 locations within the Wheeling Creek watershed. The site locations have been mapped using Geographical Information Systems (GIS). Responsibilities included leading a field crew of biology seniors on sampling/collection activities, sorting macroinvertebrate samples, analyzing data, and generating reports.

-Mimic ${ }^{\mathrm{TM}}$ Project (1994-1996) - Dr. Ben Stout, WJU / Dr. Linda Butler \& Dr. Mary Wimmer, West Virginia University /Dr. Richard Reardon, U.S. Forest Service, Department of Forest Health and Management. This study was conducted to determine potential deleterious effects of a new gypsy moth growth regulator on non-target aquatic macroinvertebrates. Additional information was collected in the laboratory at WJU to determine potentials effects of the growth regulator on selected aquatic arthropods. Data was analyzed and reports were generated.

-Mendel Club Moderator (1993-1996):

(4 yrs) Acting as the "biology" club for WJU, the Mendel Club sponsored numerous educational field trips and outdoor events.

-Haig Scholarship Committee (1995-1996)

Competitive student scholarship for science and psychology research projects.

-Mentoring (Summer 1994-1995)

Upward Bound (math/science institute) high school students during the summers of 1994 and 1995. Students were guided through a short science project which they presented to faculty and peers. 
-Substitute Lecture Instructor:

Ecology \& Ecology Laboratory, General Biology, Processes of Biology

\section{TMI Environmental Services}

-Project Leader/Environmental Scientist (1989-1992)

Responsibilities included hydrogeological investigations at chemically impacted and potentially impacted sites. Skills developed included report generation, soil and groundwater sampling, monitor well installation, sample analysis via gas chromatography, site remediation, and computer assisted drafting including groundwater flow modeling

\section{U.S. Fish \& Wildlife Service - Lamprey Control}

-Field Technician (subcontract--16 weeks, Spring 1988-1989)

In an effort to determine potential impacts on lamprey populations due to control techniques, several traps were monitored daily. The traps were located on the walls of a dam located along the St. Joseph River, Berrien Springs, Michigan. Lamprey were removed and measured while remaining fish were identified and released. Weekly reports were forwarded to the U.S. Fish and Wildlife Service.

\section{Central Michigan University}

-Graduate Student / Graduate Teaching Assistantship (1984-1987)

General Biology Laboratories

Comparative Anatomy Laboratories

Quantitative Biology Laboratories

Substitute Teaching -Animal Behavior, Anatomical Preparations, Human Biology

Personal Research Projects:

"Social Behavior Alterations in Testosterone-Injected Chickens during Growth and Development"

"A Laboratory Comparison of the Survivorship of Two Mosquito Larvae (Wyeomyia smithi -a Pitcher Plant mosquito- and Aedes sp. -a common puddle mosquito) Placed in Various PH Solutions"

"A Determination of the Animal Species Associated with Red-Backed Salamanders (Plethodon cinereus) on Beaver Island, Michigan" 


\section{Lake Superior State University}

-Undergraduate Biology Student (Senior Seminar Research Project)

"The Effect of Various Doses of Ionizing Radiation on the Germination and Subsequent Growth of Several Herbaceous Plants"

\section{XRI Testing Incorporated}

-Assistant Radiographer / Level-I, X-ray Technician, Pratt/Whitney Aircraft certified (Summers 1980-1987; Full-time 1987-1989) 